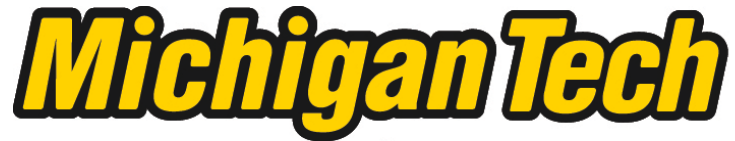 \\ Michigan Technological University Create the Future Digital Commons @ Michigan Tech
}

Dissertations, Master's Theses and Master's Reports - Open

Dissertations, Master's Theses and Master's

Reports

2005

\section{Active surgical positioning device for a cochlear implant electrode array}

Benjamin Y. Arcand

Michigan Technological University

Follow this and additional works at: https://digitalcommons.mtu.edu/etds

Part of the Mechanical Engineering Commons

Copyright 2005 Benjamin Y. Arcand

\section{Recommended Citation}

Arcand, Benjamin Y., "Active surgical positioning device for a cochlear implant electrode array", Dissertation, Michigan Technological University, 2005.

https://doi.org/10.37099/mtu.dc.etds/348

Follow this and additional works at: https://digitalcommons.mtu.edu/etds

Part of the Mechanical Engineering Commons 


\title{
An Active Surgical Positioning Device for a Cochlear Implant Electrode Array
}

\author{
By \\ BENJAMIN Y. ARCAND
}

\begin{abstract}
A DISSERTATION
Submitted in partial fulfillment of the requirements for the degree of DOCTOR OF PHILOSOPHY

(Mechanical Engineering-Engineering Mechanics)

MICHIGAN TECHNOLOGICAL UNIVERSITY 2005
\end{abstract}

Copyright $(\odot$ Benjamin Y. Arcand 2005 


\section{MICHIGAN TECHNOLOGICAL UNIVERSITY}

\section{Department of Mechanical Engineering-Engineering Mechanics}

This dissertation, “An Active Surgical Positioning Device for a Cochlear Implant Electrode Array," is hereby approved in partial fulfillment of the requirements for the degree of DOCTOR OF PHILOSOPHY in the field of Mechanical Engineering-Engineering Mechanics.

Signatures:

Dissertation Advisor

Dr. Craig Friedrich

Committee

Dr. Michele Miller

Dr. Tammy Haut-Donahue

Dr. Paul Bergstrom

Department Chair

Dr. William W. Predebon

Date 


\section{Acknowledgments}

The long journey leading to the completion this body of work followed a path abounding with the contributed efforts of untold numbers of colleagues, friends, and family. This undertaking would have been unthinkable if it had not been for the accumulated effect of their encouragement, support, and understanding.

I would especially like to express my sincere gratitude to Dr. Craig Friedrich for giving me the opportunity to work with him on this challenging and rewarding line of research. His knowledge, talents and guidance together with a seemingly limitless well of patience were more than I could have asked for in an advisor.

My utmost appreciation and gratefulness goes to my family for their unwavering belief in my abilities throughout my life and especially over these challenging years as I pursued my graduate degree. The lessons that I learned from them are the foundation for everything I accomplish today.

A special thanks to my fellow graduate students Nikhil Butala, Sudeep Shyamsunder and Shailesh Gugale for whom I worked closely with on many aspects of this project. Their hard work and commitment were essential to its success.

Finally, to Alexis, words cannot convey the magnitude of you what you gave to me. Your boundless love and selfless generosity will forever be a constant wonder and a gift to guide me throughout my life.

This work was supported primarily by the Engineering Research Centers

Program of the National Science Foundation under award number EEC-9986866. 


\begin{abstract}
Cochlear implants have been of great benefit in restoring auditory function to individuals with profound bilateral sensorineural deafness. The implants are used to directly stimulate auditory nerves and send a signal to the brain that is then interpreted as sound. This project focuses on the development of a surgical positioning tool to accurately and effectively place an array of stimulating electrodes deep within the cochlea. This will lead to improved efficiency and performance of the stimulating electrodes, reduced surgical trauma to the cochlea, and as a result, improved overall performance to the implant recipient.
\end{abstract}

The positioning tool reported here consists of multiple fluidic chambers providing localized curvature control along the length of the attached silicon electrode array. The chambers consist of $200 \mu \mathrm{m}$ inner diameter PET (polyethylene therephthalate) tubes with $4 \mu \mathrm{m}$ wall thickness. The chambers are molded in a tapered helical configuration to correspond to the cochlear shape upon relaxation of the actuators. This ensures that the optimal electrode placement within the cochlea is retained after the positioning tool becomes dormant (for chronic implants).

Actuation is achieved by injecting fluid into the PET chambers and regulating the fluidic pressure. The chambers are arranged in a stacked, overlapping design to provide fluid connectivity with the non-implantable pressure controller and allow for local curvature control of the device. The stacked tube configuration allows for localized curvature control of various areas along the length of the electrode and additional stiffening and actuating power towards the base. Curvature is affected along the entire length of a chamber and the result is cumulative in sections of multiple chambers. The actuating chambers are bonded to the back of a silicon electrode array. 


\section{Summary of results:}

- A manufacturing process for multiple chamber insertion tools has been developed and prototypes have been fabricated at several scales.

- Testing cavities for insertion tests of the tool have been fabricated at several scales.

- Insertion tests have been conducted that demonstrate this type of actuation being appropriate for a cochlear insertion tool.

- The tool has been integrated with both a high density silicon electrode array and an electrokinetic pump.

- A predictive theory combining FEA results and mathematical models has been developed for the actuation of the chambers.

- A lithographic process to build the insertion tool has been developed and tested to improve the performance and manufacturability of the tool. 


\section{Table of Contents}

List of Figures $\quad$ iv

$\begin{array}{lll}1.0 & \text { Introduction } & 1\end{array}$

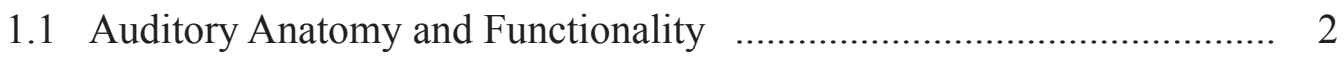

1.1.1 The Outer Ear ...................................................................... 2

1.1.2 The Middle Ear ............................................................... 4



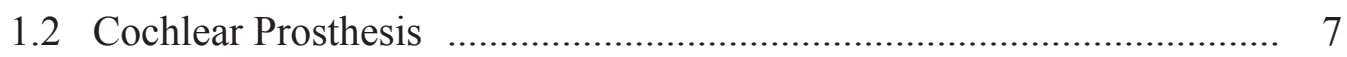

1.2.1 History of Cochlear Implants ........................................... 7

1.2.2 Components of a Cochlear Prosthesis f................................... 8

1.2.3 Surgical Placement and Prosthesis Performance f.................... 9

1.3 Commercial Cochlear Implants ................................................ 12

1.3.1 Insertion Strategies of Commercial Implants f.................... 12

1.4 An Articulated Cochlear Insertion Tool ........................................ 16

2.0 Cochlear Geometry and Modeling 19

2.1 Geometry of the Guinea Pig Scala Tympani ….................................. 19

2.1.1 Voie-Spelman Three-Dimensional Cochlear Reconstruction .. 19

2.1.2 Parametric Formulation for the Guinea Pig Scala Tympani ... 21

2.1.3 Guinea Pig ST Insertion Testing Cavities f......................... 26

3.0 Fluidically Actuated Cochlear Implant Design 29

3.1 Silicon Electrode Array ............................................................ 29

3.1.1 Electrode Array (Probe) Design ........................................... 29

3.1.2 Probe Level Sensors ..................................................... 32 
3.2 Articulated Electrode Array Backing Tool ……………………........... 33

3.3 Actuation Principle of the Fluidic Chambers …….............................. 36

3.3.1 Experimental Results of Fluidic Chamber Actuation ............. 38

3.3.2 FEA Modeling of the Fluidic Chambers ……………………... 47

3.3.3 Theory for Mechanisms of Actuation ……………………..... 53

3.4 Computational Model for Insertion Tool Actuation ………………..... 57

3.4.1 Module 1: Cross Section Deformation ……………………... 58

3.4.2 Module 2: Deformation from the Pressure Differential ......... 59

3.4.3 Module 3: Overall Chamber Centerline Deformation ............ 60

3.4.4 Comparison of the Model With Experimental Measurements 62

3.4.5 Creating a Model Space Using DOE ……………………….... 63

3.4.6 Summary of Computational Methodology ……........................ 67

3.5 Method for Applying Computational Model to an Insertion System .. 68

\subsection{Fabrication Methods and Results}

4.1 Development of the Fabrication Process ……………………………... 69

4.2 Fabrication Steps for PET Actuation Chambers …………………..... 70

4.2.1 Step 1: Pressure inlet port ……………………………….... 70

4.2.2 Step 2: Chamber flattening ……………………….............. 71

4.2.3 Step 3: Chamber end sealing ……………………………..... 74

4.2.4 Step 4: Chamber bonding and electrode array integration ...... 74

4.2.5 Step 5: Inducing the final helical shape on the implant .......... 77

4.2.6 Supplementary Step: Dual diameter chamber ……………..... 77

4.3 Backing Prototypes …………………………............................. 79

4.3.1 Early single chamber $3 \mathrm{x}$ insertion tools …………................... 79

4.3.2 Multiple chamber $3 x$ insertion tools …………....................... 81

4.3.3 Integration of the insertion tool and silicon electrode array $\quad \ldots \quad 82$ 


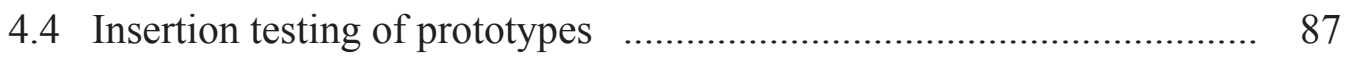

4.4.1 3x scale insertion tests [32] …………………...................... 87

4.4.2 1x scale insertion tests …………………………………... 87

4.4.3 Integration of electrokinetic pumps ………………………..... 89

5.0 Next Generation Fluidic Actuator Manufacturing Process 97

5.1 Lithographic Process for High Aspect Ratio Flexible Fluidic Cavities 97

5.2 Results of Fabrication for the Lithographic Process ……................. 102

$\begin{array}{ll}\text { 6.0 Conclusions } & 113\end{array}$

6.1 Recommendations for Future Research ………………………....... 114

$\begin{array}{ll}\text { References } & 115\end{array}$

Appendix A: Cochlear Geometry and Programs \& Modeling 118

Appendix B: Machining Programs for the Micromilling Machine 124

Appendix C: Data from Chamber Actuation Expermients 136

Appendix D: Programs for Modeling Bourdon Actuation of Chambers 140

Appendix E: Interferometer Plots of a Polyimide Device 153 


\section{List of Figures}

Figure 1.1 Anatomy of the human ear. ............................................................ 3

Figure 1.2 Detailed anatomy of the middle ear. ................................................ 3

Figure 1.3 Anatomy of the human cochlea. …............................................... 5

Figure 1.4 Anatomical cross section of the Organ of Corti. ................................ 5

Figure 1.5 Major components of a cochlear prosthesis. .................................. 9

Figure 1.6 Cochlear Corporation's Nucleus 24 Contour Implant with pre-curled electrode array (inset) ...................................................... 13

Figure 1.7 (left) Nucleus 24 Contour implant demonstrating the stylette. .......... 14

Figure 1.8 (above) CLARION HiFocus (a) electrode positioner (b) precoiled im-



Figure 1.9 MED-EL Combi 40+ implant (top) and headset (bottom). ............... 15

Figure 1.10 Diagram of an articulated cochlear insertion tool. ........................... 17

Figure 2.1 (a) Cross sections of a guinea pig (b) and a human cochlea. ............. 20

Figure 2.2 Voie-Spelman data of a guinea pig scala tympani........................... 20

Figure 2.3 Direct measurement of the cochleostomy position. .......................... 22

Figure 2.4 Coordinate system for ST mathematical model. ............................. 22

Figure $2.5 \quad$ (a) Cross Sections of the ST (b) ST reconstructed from the parametric



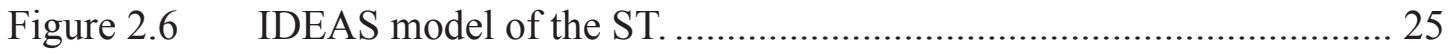

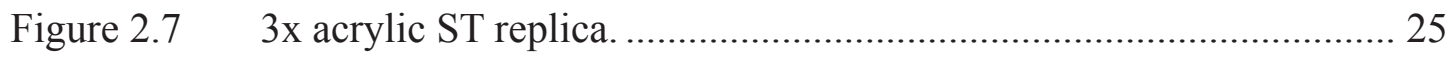

Figure 2.8 Machined 1x scale single-turn ST insertion cavity......................... 27

Figure 3.1 Layouts for the high density silicon electrode arrays. ..................... 30

Figure 3.2 High-density monolithic cochlear electrode array............................ 31

Figure $3.4 \quad$ Probe level lace lateral touch sensors. .......................................... 33

Figure 3.3 Probe level peizoresistive strain gages \& tip sensor (inset). ............. 33 
Figure 3.5 Basic manufacturing steps and arrangement for the overlapping chamber design of the insertion device. ........................................... 35

Figure 3.6 A Bourdon tube as applied to a C-type pressure gage. ...................... 37

Figure 3.7 Variable definitions for Bourdon tube element and cross section geom-

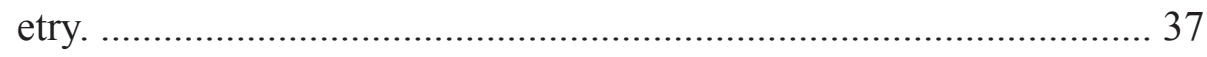

Figure 3.8 Chamber actuation at 0,15 and 30 psi (scale in $\mathrm{mm})$. ..................... 40

Figure 3.9 Measuring chamber radius. ....................................................... 40

Figure 3.11 Chamber radius vs Pressure with a fitted 2nd order polynomial and computed confidence and probability intervals. ........................... 42

Figure 3.10 Radius increase vs pressure of several 1x actuation chambers .......... 42

Figure 3.12 Chamber actuations for several starting radii. ................................ 45

Figure 3.13 Loss of springback and radius achieved with vacuum. .................... 45

Figure 3.15 Cross section profiles at a) $0 \mathrm{kPa}$ and b) $70 \mathrm{kPa}(10 \mathrm{psi}) . \ldots \ldots \ldots \ldots \ldots \ldots . . . . . . . .46$

Figure 3.14 Interferometric microscope measurement of a PET chamber cross sec-

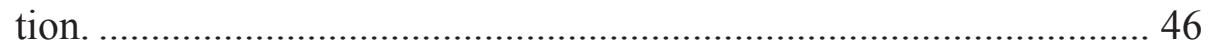

Figure 3.16 Chamber cross section radius during pressurization. ....................... 46

Figure 3.17 FEA model setup for chamber actuation $(\mathrm{R}=1000 \mu \mathrm{m})$. ................... 50

Figure 3.18 FEA results for a $10^{\circ}$ semicircular section with a $1000 \mu \mathrm{m}$ radius..... 50

Figure 3.19 Plastic strain in the edge location for the FEM. .............................. 52

Figure 3.20 Actuated radius of curvature for several FEA chamber sections of varying starting radius versus experimental results. ...................... 52

Figure 3.21 Overview of modules in the computational model. .......................... 58

Figure 3.22 Program flow for chamber actuation computational model. ............. 61

Figure 3.23 Results from the computational model vs. experimental results....... 62

Figure 3.24 DOE model of final chamber radius by input parameters starting radius, wall thickness, and low and high pressures. ......................... 66

Figure 4.1 Grinding of a steel hypo and the resulting beveled edge. .................. 72 
Figure 4.2 Transition from round steel hypo to flat PET tubing.......................... 72

Figure 4.3 a) Macor flattening fixture and b) setup for flattening. ....................... 72

Figure 4.4 Chamber sealing apparatus and closeup view of nichrome wire. ...... 75

Figure 4.5 Silicon probe with wrongly directed curvature. ................................. 75

Figure 4.6 Vacuum tweezer set for probe manipulation. ..................................... 75

Figure 4.7 Helical brass winding mandrel. .................................................... 78

Figure 4.8 Technique for drawing PET tubing to make dual diameter chambers. .. . .78

Figure 4.10 a) An early $3 \mathrm{x}$ insertion tool and b) its actuation ability .................. 80

Figure 4.11 Two views of a $3 \mathrm{x}$, three chambered insertion tool. ............................ 80

Figure 4.9 A dual diameter PET chamber fabricated by drawing........................ 80

Figure 4.12 a) Pressure regulating system and b) attached to a three chambered prototype.

Figure 4.13 Actuation of a three chambered insertion tool with successive pressurization of each chamber to $30-40$ psi.................................................. 83

Figure 4.14 A 3x single chambered insertion tool with electrode array. ............... 83

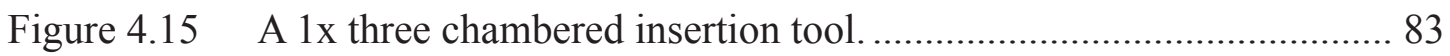

Figure 4.16 A 1x insertion device integrated with a silicon electrode array and pro-

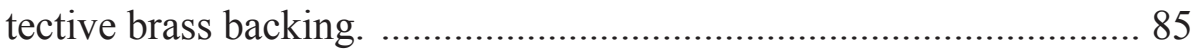

Figure 4.17 Protective brass backing attached to a silicon electrode array. .......... 85

Figure 4.18 Modiolus hugging insertion tool integrated with an electrode array shown above 1x ST model. .............................................................. 88

Figure 4.20 A series of photographs from an insertion test for a 1x scale tool. .... 88

Figure 4.19 Insertion test for a 3x single-chambered device into an acrylic ST



Figure 4.21 Insertion test for a $1 \mathrm{x}$ device with an integrated silicon electrode array. .90 
Figure 4.22 Diagram of the principle of electroosmotic flow.

92

Figure 4.23 Electrokinetic pump developed at the University of Michigan Micro-

fluidics Lab. 92

Figure 4.24 Performance plots for the UofM EK pump. 94

Figure 4.25 Actuation time of a single chamber tool using the UofM EK pump .. 94

Figure 4.26 Redesigned EK pump with attached single chamber insertion tool and electrode array. 95

Figure 4.27 Actuation times of a single chamber insertion tool for the redesigned EK pumps. 95

Figure 5.1 Lithographic process steps for a polyimide insertion tool. 99

Figure 5.2 Conceptual diagram for an insertion tool fabricated using the lithography polyimide process. 101

Figure 5.3 A minuterized three EK pump bank. 101

Figure 5.4 Base and top patterns for the polyimide films. All units are reported in micrometers. 104

Figure 5.5 Quartz core mask pattern for the PMMA layer. 104

Figure 5.6 The deep UV lamp housing and ballast bank 105

Figure 5.7 Deep UV lamp housing mounted and operating on the EVG aligner .... 105

Figure 5.8 Images of a fabricated polymide device with PMMA core on the substrate surface. From top to bottom: backend with port opening to polyimide core, chamber tip attached to polyimide leash, and the handle with adjacent target. 107

Figure 5.9 Interfermetric measurements of the port and tip areas for a polyimide device. 108

Figure 5.10 SEM image of a cured polyimide device chamber that has been sliced open to view the interior. 108 
Figure 5.11 Brass manifold and steel hypo used to connect fluidic pressure to the polyimide device's port.

Figure 5.12 Cured and shaped polyimide device. ......................................... 110

Figure 5.13 Close up shot of the bonded brass manifold and device port. .......... 110 


\section{List of Tables}

Table 2.1 Translations and Rotations of the ST Data ......................................23

Table 2.2 Parametric Equations for the Geometry of a Guinea Pig ST ..............24

Table 3.1 DOE Parameters and Levels ...........................................................64

Table 3.2 DOE Run Settings and Response Results ......................................65 


\subsection{Introduction}

Cochlear implants have become an accepted and successful treatment for profound, bilateral, sensorineural deafness in both children and adults. Over 100,000 recipients of implants worldwide have experienced restoration of useful levels of auditory function. The widespread use of multi-channel devices in recent years has contributed greatly to the performance experienced by users of cochlear prostheses.

The tonotopic organization within the cochlea allows electrodes in multi-channel devices to stimulate localized sub-populations of auditory nerves responsible for perception of various frequencies. The desired effect is to selectively stimulate the auditory neurons in a way that mimics the ear's healthy functionality. Essential to effective and efficient localized stimulation of the auditory nerves is the position of the stimulating electrodes within the cochlea. However, the small size, delicate internal structures and helical shape of the cochlear chambers complicates the matter of precise positioning of the implant.

There are a number of commercial cochlear implants in clinical use today incorporating multiple electrodes and multi-channel stimulation methods. Each incorporates its own method for surgical implantation and positioning within the cochlea and each method has its advantages and disadvantages. A common disadvantage of all the commercial implants is a loss of position control once the device is inserted into the cochlea. Movement and position control of the inserted portion of the implant is achieved only through manipulation of the extraneous portion

of the implant by the surgeon's hand. The result is that the typical insertion reaches less than half the possible insertion depth into the cochlea and positioning is poor. Cochlear damage leading to further auditory nerve degeneration is not uncommon. 
A proposed solution to resolve this problem is an electrode array incorporating hydraulic actuation chambers that can actively control the shape of the combined device. By changing the pressure to the fluidic chambers, the surgeon (or control system) can alter the shape of the device to match the shape of the cochlea. This would reduce contact pressures on the delicate internal structures of the cochlea mitigating operative trauma. Furthermore, lowered contact force within the cochlea would also reduce the friction between the device and the cochlea wall allowing for a deeper insertion that reaches more auditory nerves increasing perceived frequency bands.

\subsection{Auditory Anatomy and Functionality \\ The anatomy of the ear is commonly divided into three portions: The outer, middle and inner ear [1,2] (Fig. 1.1).}

\subsubsection{The Outer Ear}

The outer ear is composed of the familiar pinna (or auricle) composed of cartilage, the cartilaginous and bony portions of the ear canal and the tympanic membrane. The pinna allows the source of sound to be localized between the front and back of an individual. The ear canal channals impinging sound energy into the inner structures as well as secreting cerumen to remove dust and debris. The length of the adult ear canal is approximately $25 \mathrm{~mm}$ giving it a resonance frequency of $3400 \mathrm{~Hz}$, an important frequency for understanding speech.

The tympanic membrane, located at the end of the ear canal, is composed of three layers with the outermost layer forming a continuous structure with the ear canal. The lower portion of the membrane called the pars tensa actively vibrates in response to sound traveling down the ear canal. The vibrational energy is in turn transferred to the structures of the middle ear. 




Figure 1.1 Anatomy of the human ear.

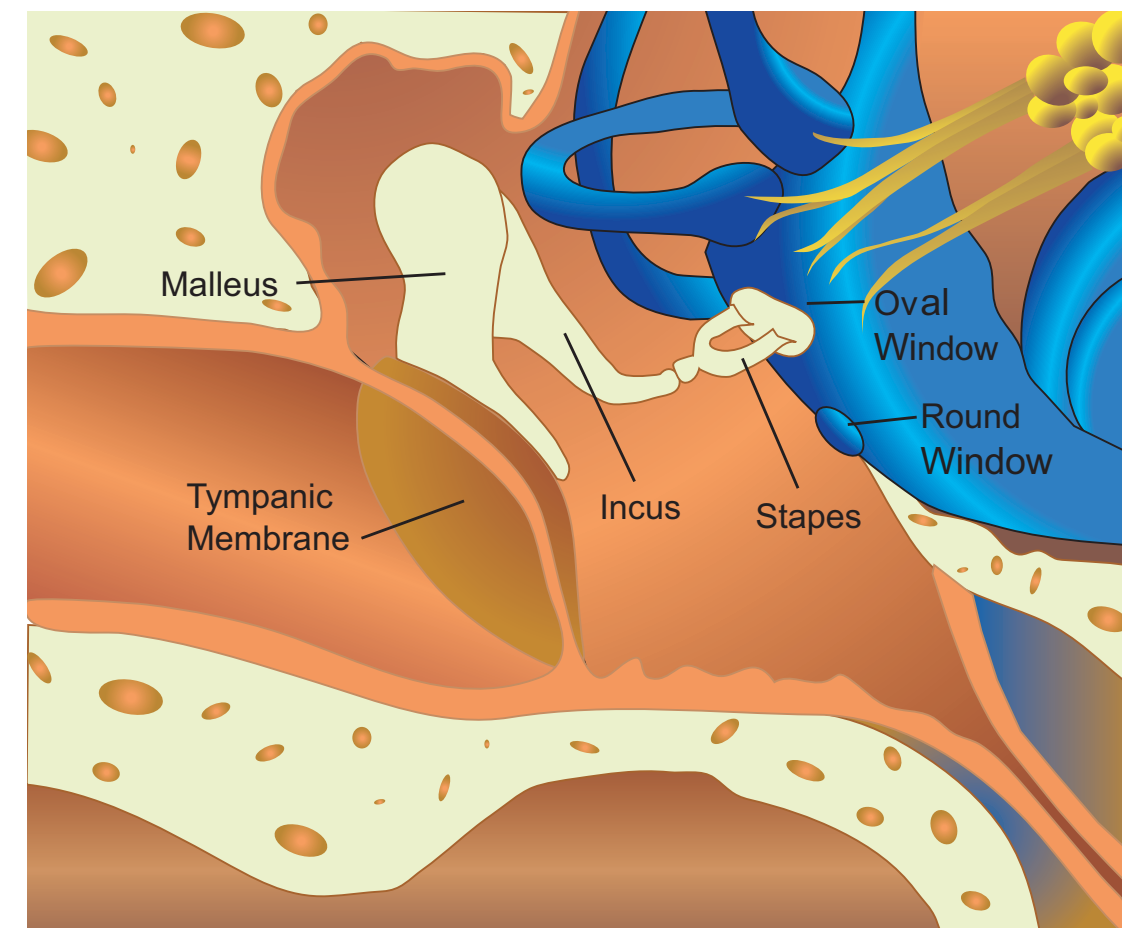

Figure 1.2 Detailed anatomy of the middle ear. 


\subsubsection{The Middle Ear}

The function of the middle ear is to serve as an impedance matching mechanism between the air of the ear canal and the perilymph fluid of the inner ear. To accomplish this, three small bones work in concert to transfer sound energy to the fluid of the cochlea (Fig. 1.2).

The long, bony process of the most lateral bone, called the malleus, is attached to the innermost layer of the tympanic membrane. On the opposite end of the malleus is the slightly shorter incus. The length difference between the two bones provides a slight levering advantage boosting sound energy by about $2.5 \mathrm{~dB}$. The innermost bone of the middle ear, the stapes, has a footplate seated in the oval window of the cochlea and a superstructure attached to the incus. Because the area of the tympanic membrane is much larger than the footplate of the stapes, sound energy is given a hydraulic advantage of over $20 \mathrm{~dB}$ before entering the fluid-filled spaces of the cochlea.

\subsubsection{The Inner Ear}

The cochlea is a snail-shaped, auditory organ responsible for converting the mechanical energy of sound waves to an electrical signal that can be interpreted by the brain (Fig. 1.3). Vibrational energy enters the cochlea through the oval window from the footplate of the stapes. The oval window is located at the most basal end of the scala vestibuli, one of the three partitions within the cochlea, and separates the middle ear from the perilymph fluid of the inner ear.

Located between the scala vestibuli and scala tympani (ST), the scala media contains the organ of corti (Fig. 1.4) which is responsible for converting the vibrational energy in the fluids to neural energy. The Organ of Corti consists of the tectorial and basilar membranes, the inner and outer hair cells and supporting cells. 


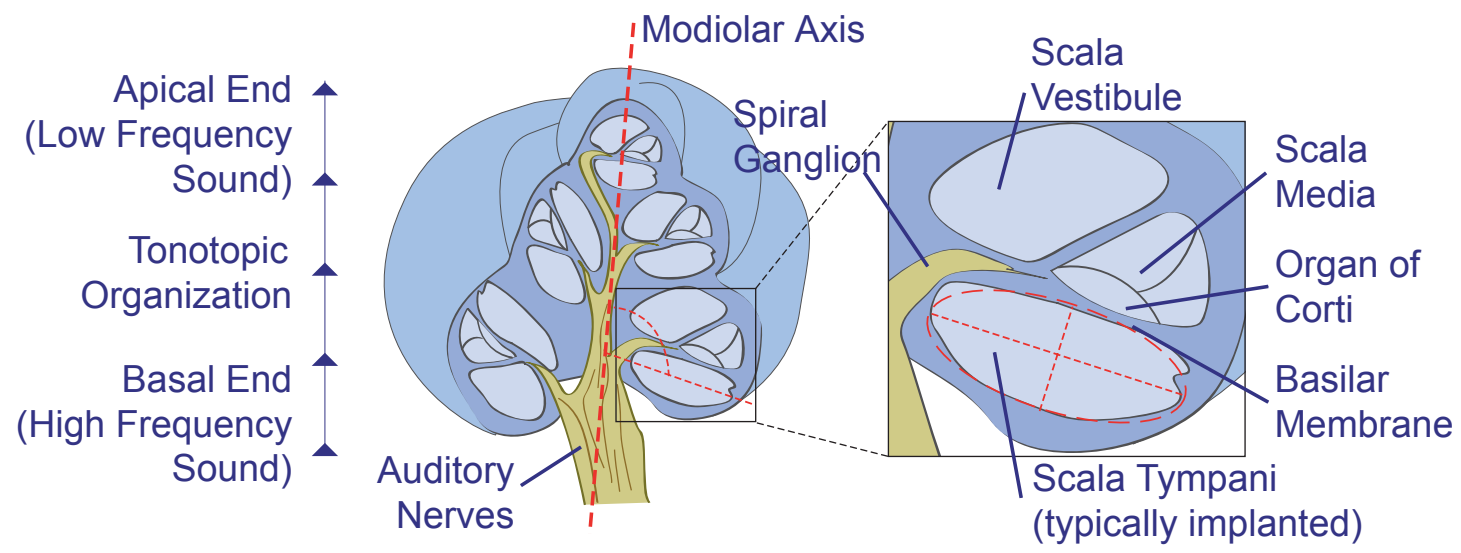

Figure 1.3 Anatomy of the human cochlea.

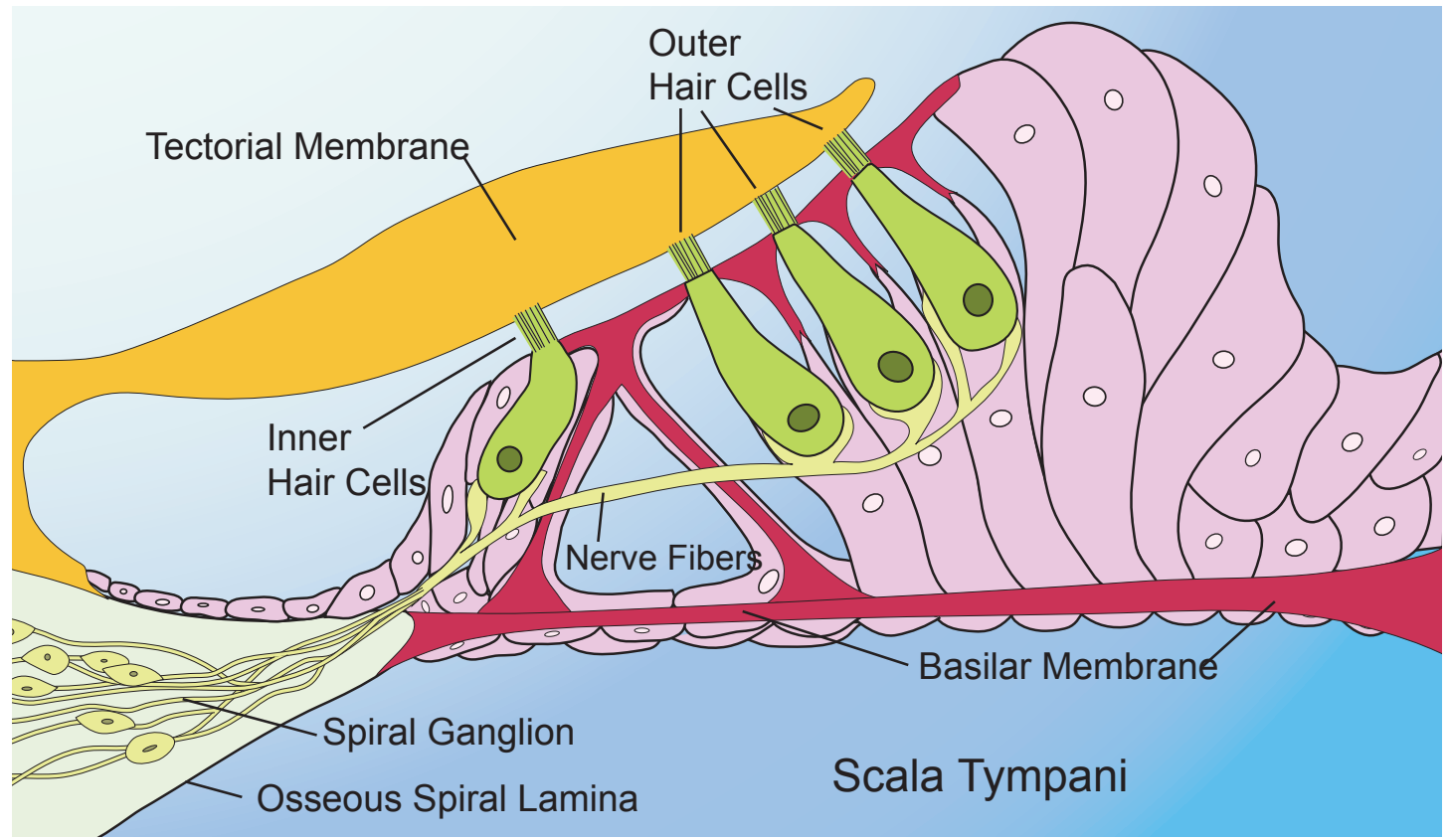

Figure 1.4 Anatomical cross section of the Organ of Corti. 
The basilar membrane is a ribbon-like structure that separates the scala tympani from the scala media and upon which rests the Organ of Corti. It is narrower and stiffer at its basal end and wider and more compliant at the apical end. These structural and stiffness gradients cause the membrane to be attuned to high frequencies at the basal end and low frequencies as it progresses to the apex giving rise to a tonotopic organization of sound perception within the cochlea.

The inner and outer hair cells are embedded in the delicate, gelatinous tectorial membrane. The tuned action of the basilar membrane causes a shearing motion and depolarization of the hair cells which respond with an on/off neural impulse to the brain through the auditory nerves that is interpreted as sound.

The approximately 3,500 inner hair cells are responsible for about $95 \%$ of the innervation from the acoustic portion of the auditory nerve and are the primary source of auditory perception. The outer hair cells number approximately 12,000 and contain muscle like filaments that contract and aid in tuning the response of the basilar membrane.

Nerve fibers from hair cells extend to the nerve cell bodies (the spiral ganglion) located in the osseous spiral lamina, a delicate, bony shelf extending from the axis of the cochlea and partially separating the scala vestibule and scala tympani. From there, the auditory nerve fibers extend down the axis of the cochlea towards its base and continue to the brain.

Trauma, degeneration due to disease, and deformities can both disrupt the delicate mechanisms of sound transduction within the ear and cause hearing loss. Irreversible damage to the hair cells of the cochlea results in sensorineural hearing loss. Since these cells do not regenerate and the mechanism 
for translation of mechanical sound to a neural signal is interrupted, typical amplification devices such as hearing aids are ineffectual. Although there may be severe damage to the hair cells of the cochlea, residual nerve fibers typically survive. Through the act of applying electrical stimulation to these residual nerve fibers by means of a cochlear implant, restored auditory perception is possible.

\subsection{Cochlear Prosthesis}

Individuals with severe sensorineural hearing loss may benefit from a cochlear prosthesis. A cochlear prosthesis functions by circumventing passing all functionality of the natural ear and stimulating the auditory nerves located in the cochlea by electrical impulses. The stimulated auditory nerves then send a neural signal to the brain to be interpreted as sound.

\subsubsection{History of Cochlear Implants}

Commercial cochlear implants have only become recently available since the 1980's. However, the concept of using electrical rather than acoustic stimulation to induce an auditory response in the brain has been around since the late 1800's. In 1880, Alessandro Volta was first to report that the electrical stimulation of a metal rod inserted in his ear canal created an auditory sensation. Djourno and Eyries placed a wire on the auditory nerve of an individual undergoing surgery in 1957. The patient reported a clear auditory percept when a current was supplied to the wire. An experiment performed by House and Doyle in 1961 involved placing an electrode through the round window into the scala tympani in two adults with profound deafness. Both individuals reported clear auditory percepts and moreover they reported that loudness increased with stimulation level and pitch with variation in the rate of stimulation. These observations promoted further development towards a permanently implantable system [3]. 
The first single-channel cochlear implant was introduced in 1972 and implanted in 1000 individuals. The 3M/House cochlear implant provided many users with improved speech reading ability. The first multi-channel cochlear implant was introduced by Cochlear Corporation in 1984. Their Nucleus 22 device consisted of an implantable receiver/stimulator and a cochlear electrode array consisting of 22 banded electrodes. Since then, several other commercial implants have become available that follow similar design principles of the Nucleus device.

\subsubsection{Components of a Cochlear Prosthesis}

These cochlear prostheses consist of several major components: The external microphone, the speech processor, a transcutaneous transmitting coil and the intracochlear electrode array (Fig. 1.5). The external microphone picks up sound signals impinging on the recipient and passes the signal to the speech processing unit. The speech processor is a miniature computer that analyzes the signal and filters and maps it to corresponding electrodes of the implant.

Because of the tonotopic organization of auditory perception within the cochlea, electrodes of the implant can be positioned adjacent to nerve fibers responsible for different frequencies. The electrodes work to stimulate discrete areas along the spiral length of the cochlea, and consequently, various groups of auditory neurons responsible for different frequency perceptions. The sound, impinging on the external microphone, is divided up by frequency and the signals are sent down different channels corresponding to certain electrode groups for nerve stimulation. Rather than one signal and frequency band as in single-channel devices, information on sound is transmitted on many frequency bands to different groups of auditory neurons for processing by the brain. The result is a higher degree of frequency resolution and multiple levels of sound perception, which translates into improved speech recognition for the implant recipient. 


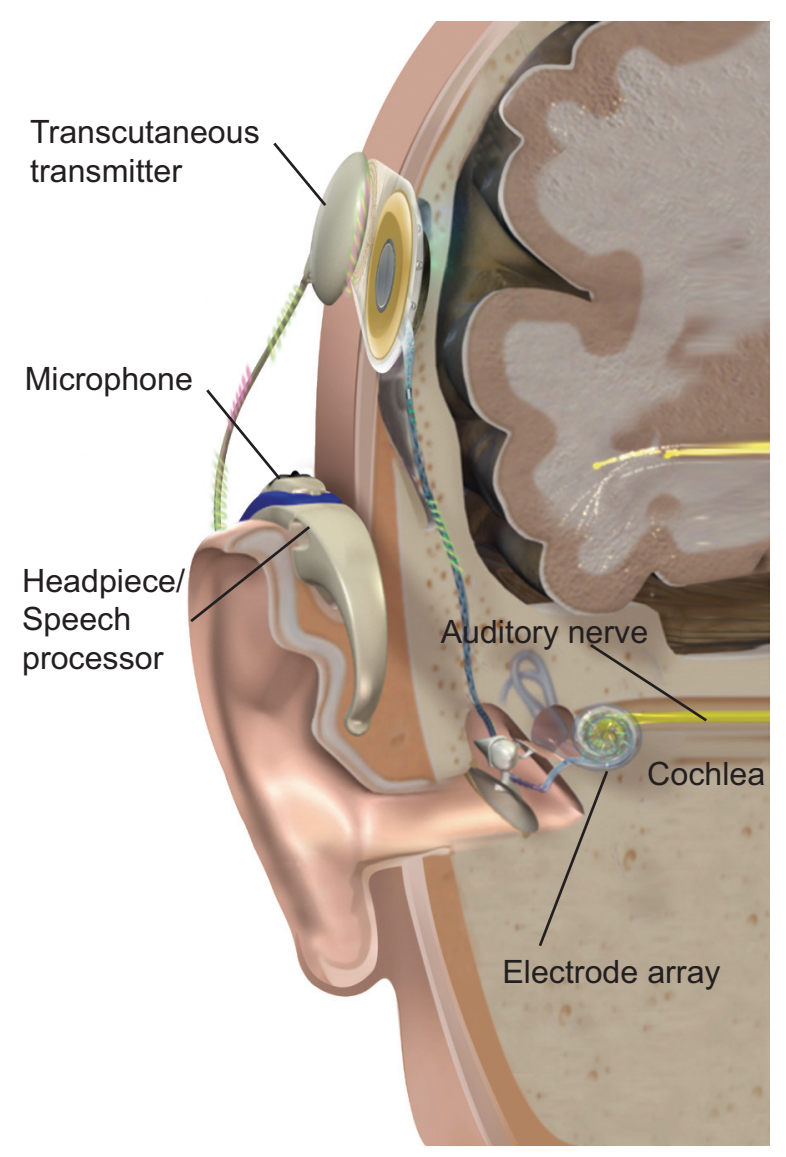

Figure 1.5 Major components of a cochlear prosthesis.

The implant itself is a long flexible probe with electrodes residing along its length. The implant is usually inserted by a surgeon through the round window of the cochlea into the chamber of the scala tympani. Inclusion of the implant destroys any residual hearing left to the recipient in that ear making cochlear implants only suitable for individuals with severe to complete bilateral deafness.

\subsubsection{Surgical Placement and Prosthesis Performance}

Numerous factors can affect the quality of performance once a device has been implanted. Two of the prevailing attributes affecting quality are electrode placement within the cochlea and trauma incurred through the insertion procedure. 
Placement of the electrode governs the characteristics of the stimulation between the auditory nerves and surrounding tissues. Damage to structures within the cochlea caused by the insertion can lead to degeneration of the spiral ganglion cells and significant loss of the remaining nerve cells available for stimulation $[4,5]$.

Studies have shown that to maximize the effectiveness of localized stimulation with the multichannel devices, precise placement of the electrodes within the scala tympani is of utmost importance [6-10]. The auditory nerves responsible for hearing are located in a structure within the central axis of the cochlea called the modiolus. From the modiolus, peripheral dendrites extend into the cochlear chambers through a bony shelf between the scala media and scala tympani. Shepard (et al.) demonstrated that optimal electrode placement for reduced stimulation thresholds, is adjacent to the peripheral dendrites [11]. He added that the fragile nature of the structures housing the peripheral dendrites creates a prohibitive situation for placing electrodes nearby. A conciliatory position close to the modiolus has been found to not only increase the spatial selectivity of stimulating electrodes, but also to reduce current thresholds required for stimulation and increase the dynamic range $[9,11]$.

Reducing current thresholds is important for two reasons. First, current density at the electrode surfaces is associated with the creation of harmful products through electrochemical processes [12]. These harmful products could potentially lead to further irreversible deterioration of the cochlea. Secondly, power requirements for the device would be reduced if current thresholds were lowered. This would mean smaller more convenient battery sizes with longer charge life and fewer recharging sessions for the user of the implant. Additionally, reduced current in the electrodes would decrease occurrences of side effects such as facial stimulation [13]. 
Another factor affecting the quality of performance by multi-channel implants is the depth of insertion into the cochlea [6,7,14]. Considering the tonotopic organization within the cochlea, deeper insertion can reach the more apical auditory nerves and provide middle and lower frequency perception, which is essential for speech recognition. Also, this region of the inner ear is usually last to undergo degeneration, making it a prime candidate for stimulation by an implant [6].

Implants are most often inserted in the basal end of the cochlea, either through the round window or through a cochleostomy. To reach the apical end of the cochlea, and the nerves responsible for low frequency sound, the implant must navigate the long, narrow, helical-shaped corridor of the scala tympani. Implants in use today typically employ electrodes embedded in a flexible, probe-like structure. Upon insertion, they use the internal walls of the cochlea to help guide themselves deeper within the scala tympani. This method can cause damage to the delicate internal structures of the cochlea promoting nerve degeneration and further loss of hearing potential in a patient [15]. Friction between the implant and the walls impedes deep insertion towards the apex. Insertions of these devices typically achieve less than half $\left(360-400^{\circ}\right)$ of the total angular depth of the cochlea possible.

Moreover, by using the internal structures of the cochlea for insertion, the probe has a tendency to migrate towards the outer wall away from the nerve fibers. To overcome this, some implant designs have been developed with molded, pre-curled shapes that use additional stiffening structures to hold them straight for the initial stages of the insertion. This can complicate the insertion procedure in that the probe's additional stiffness can more easily cause damage while the curvature upon release of the stiffening structure is not easily controlled. While the new models have improved 
proximity of the electrodes to the modiolus, further improvements are needed.

\subsection{Commercial Cochlear Implants}

In the United States, there are three FDA approved cochlear implant systems. These three systems (Nucleus, Clarion and Combi $40+$ ) have many similar features and are also consistent with each other in the categories of cost and performance.

The three systems are all able to provide multi-channel stimulation using multiple electrodes inserted into the scala tympani as opposed to single-channel stimulation. Each of the systems utilizes transcutaneous communication between the implanted and worn portion of the prosthesis. The externally worn hardware uses an antenna to transmit radio frequency signals to the subcutaneous receiver antenna connected to the implanted portion. The radio signal is used to both power the stimulation and to control the level and type of stimulation by the intracochlear electrodes. The two antennae are held in proximity to each other through the use of magnets.

Each of the systems offers a range of different speech processing options and they all employ a similar process for programming the speech processor. In all three, telemetry systems are available to detect malfunctioning electrodes in implanted arrays. Regardless of the similarities of the systems, each has unique features that distinguish it from the other two systems.

\subsubsection{Insertion Strategies of Commercial Implants}

The Nucleus 24 Contour manufactured by Cochlear Corporation is the companies latest implant design (Fig. 1.6) and has been in use since 1998. The internal stimulator/receiver unit is thinner and more flexible than previous models giving it a lower profile on the skull. The implanted magnet can be removed if necessary to 




\section{Figure 1.6 Cochlear Corporation's Nucleus 24 Contour Implant with pre-curled electrode array (inset) [16].}

allow for magnetic response imaging (MRI). The implant features 24 stimulating electrodes distributed logarithmically along its length with the base electrodes being more widely spaced than at the tip. The electrodes are half band, occupying the side of the implant that will face inwards towards the modiolus. This helps to reduce current spread and also lowers power consumption. The implant is manufactured in a pre-coiled spiral shape to put the electrodes in closer proximity to the modiolus.

During insertion into the cochlea, the implant is held straight by a platinum stylette inserted along its length. The stylette acts as a metal spine holding the electrode array straight and is slowly removed as the insertion into the cochlea progresses (Fig 1.7).

In 1998, Advanced Bionics began using its CLARION HiFocus precoiled implant system (Fig 1.8). The implant has 16 longitudinally arranged contacts. It is the only device capable of simultaneous stimulation of multiple electrodes within the cochlea. The Clarion device is packaged in a ceramic case that is 
set into a cavity drilled in the temporal bone. The implanted magnet within the ceramic case is not removable and also it is not MRI compatible [3].

Implanting the device involves inserting the array into the cochlea and then inserting the electrode positioner to push it further into the cochlea and towards the modiolar wall. A disadvantage of this technique is that two insertions are required as opposed to one (for both the electrode array and positioner) which increases the opportunity for damage to occur within the cochlea. Secondly, considerable damage is possible if the positioner were to place pressure on the basilar membrane [18].

In 2002, the Food and Drug Administration (FDA) became aware of a possible connection between cochlear implants and bacterial meningitis [19]. Among other factors, design of the electrode array is considered a possible predisposing factor for meningitis.

In early July of that year, European and U.S. cochlear implant surgeons and experts met to discuss the incidence of meningitis occurring after cochlear implantation. That group concluded that there were more cases of meningitis

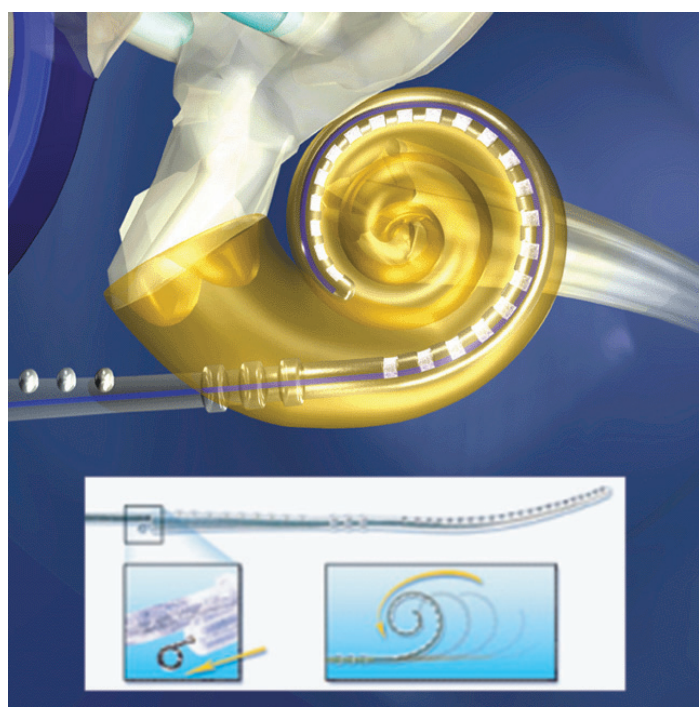



Figure 1.8 (above) CLARION HiFocus (a) electrode positioner (b) precoiled implant [17].

Figure 1.7 (left) Nucleus 24 Contour implant demonstrating the stylette [16]. 
with the CLARION device with the electrode positioner than with other cochlear implants. The organizers of that meeting recommended discontinuing use of the positioner, and the regulatory authorities of several European countries (e.g., France, Germany, and Spain) accepted those recommendations.

Consequently, Advanced Bionics agreed to discontinue use of the positioner in those countries and is marketing one of their current electrode systems (HiFocus I) without the positioner. The company also initiated a voluntary recall of any unimplanted CLARION devices in the United States, but subsequently has received FDA approval for marketing the HiFocus I electrode without the positioner [20].

The Combi $40+$ developed by MED-EL has 12 electrode pairs running along its length at $2.4 \mathrm{~mm}$ of separation between electrode pairs (Fig 1.9). The standard implant is the longest of the three cochlear implant systems with a length of $26.4 \mathrm{~mm}$. The long, thin, flexible array is inserted into the scala tympani through a cochleostomy

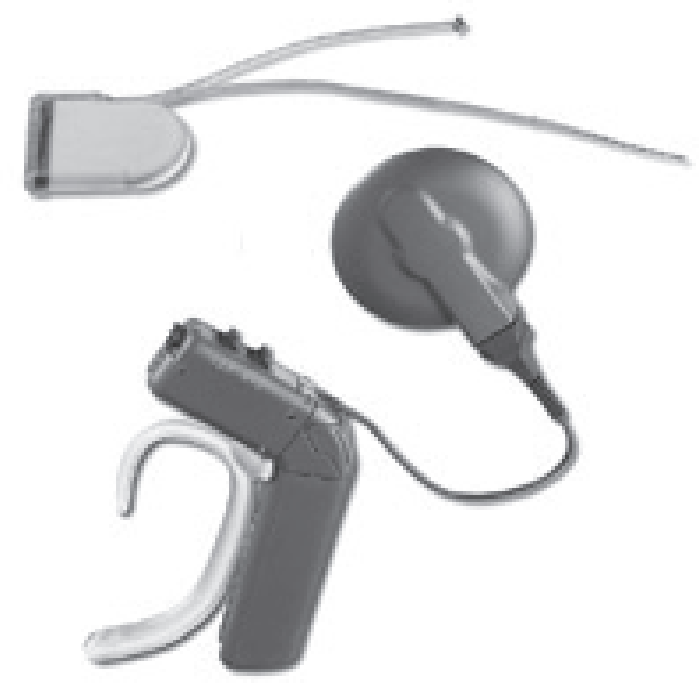

Figure 1.9 MED-EL Combi 40+ implant (top) and headset (bottom). 
and relies on the interior walls of the cochlea for curling. The array has an oval cross section, providing a weak axis for bending, to facilitate curling during insertion.

Like the Clarion system, the Combi $40+$ is housed in a ceramic case but has received FDA approval for use in MRI machines up to 0.2 Tesla [3]. The implant is configured to provide some of the highest stimulation rates of the three systems allowing up to $1515 \mathrm{~Hz} /$ channel.

\subsection{An Articulated Cochlear Insertion Tool}

Improvements to the insertion procedure can be realized by employing an implant that actively changes shape to fit the cochlea. By using fluidic actuators along the length of the implant, the shape can be changed to fit that of the cochlea during the insertion procedure.

Such an implant could reduce wall contact and friction allowing for a deeper insertion while also avoiding damage to delicate internal structures. In addition, the implant can have a complex, precoiled, tapered-helical shape to closely 'hug' the modiolus when the insertion is completed and the actuators are disabled.

The basic design of the fluidic actuators involves long, flexible chambers with a high-aspect-ratio cross-sectional shape (Fig. 1.10). The chamber has a radius of curvature along its length with the minor axis of the cross-section being parallel to the radius. Radial actuation occurs when fluidic pressure is introduced inside the chamber causing its cross-section shape to change. The response of the crosssection to the pressure is a tendency towards becoming more circular, the minor axis of the cross-section lengthens while the major axis decreases. The change in crosssection shape alters the bending and stiffness characteristics of the chamber causing 
the overall curling radius to adjust in response, effecting actuation. The result is analogous to a highly elastic, large deformation, variable cross-section Bourdon tube.

This type of actuation is ideal for a cochlear implant since we would like the device to have a shape to closely hug the modiolus, but the radius of that spiral shape needs to be temporarily increased (straightened) to allow for surgical insertion. Moreover, the change in radius can be precisely controlled with a corresponding change in fluidic pressure allowing for fine adjustments to closely fit the cochlea throughout the insertion procedure.

Here, a device to achieve actuation of a cochlear electrode array to provide deep perimodiolar placement within the scala tympani is presented. In the following

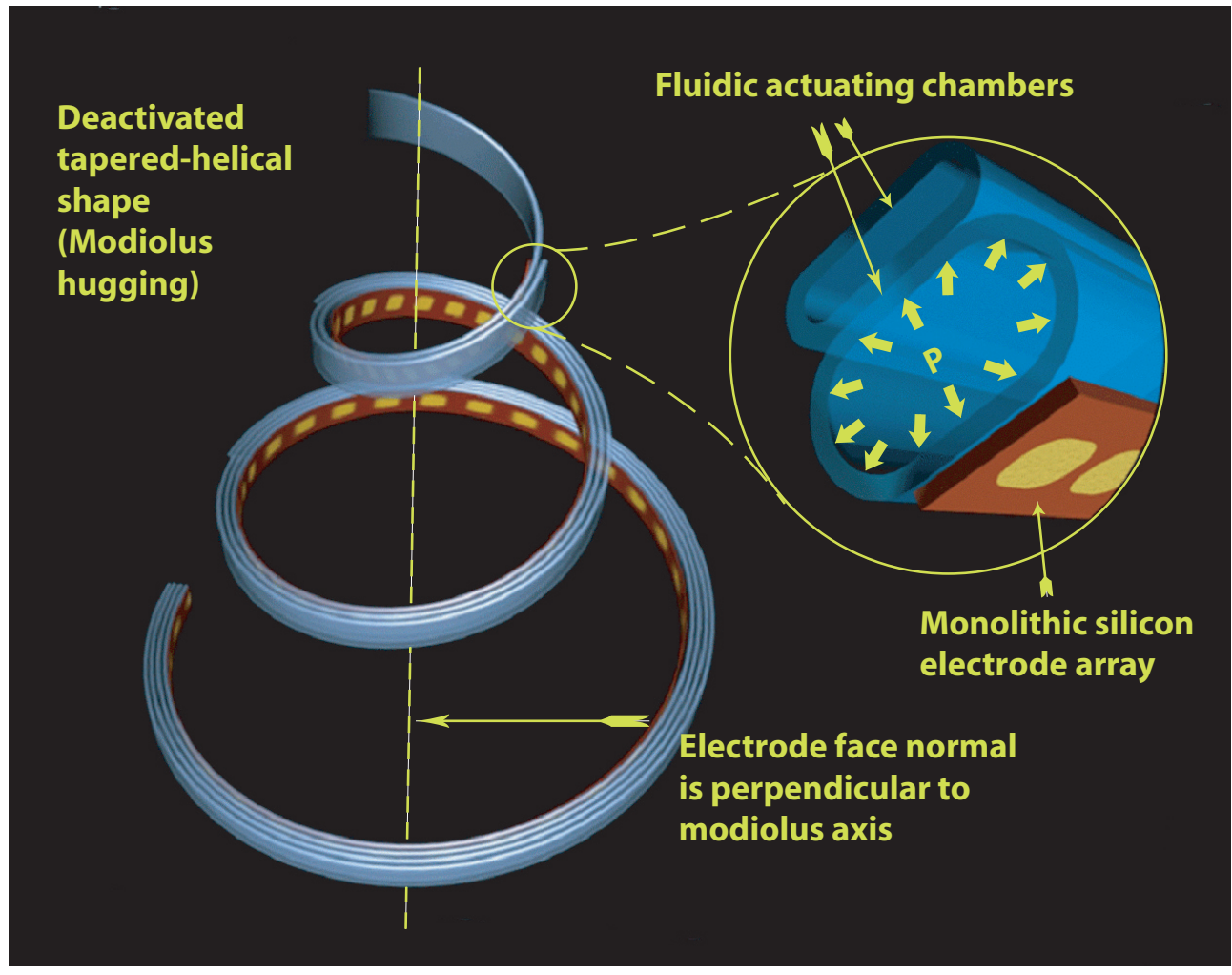

Figure 1.10 Diagram of an articulated cochlear insertion tool. 
chapters, methods will be discussed for fabricating and integrating fluidic actuation chambers with thin film cochlear electrode arrays. Experimental results are presented on the actuating properties of the chambers, as well as analytic and computational methods for modeling chambers for design and simulation purposes. Potential utility of the actuation device for surgical procedures is shown through experimental insertions of the fluidic actuation chambers into artificial cochlea models. Finally, results for a next generation fabrication technique using thin film lithography and its potential for improving both device performance and manufacturability are discussed. 


\subsection{Cochlear Geometry and Modeling}

In order to develop the dimensions and features of the actuation tool, a detailed knowledge of the geometry of the cochlea is needed. However, prior to developing an actuated implant for the human cochlea, initial tests of the implant will be performed in the cochlea of guinea pigs. The guinea pig cochlea has a similar frequency range as its human counterpart making it a good test subject for implant design, but is approximately one-third the size of the human cochlea. Additionally, the guinea pig cochlea has about 3.5 helical turns, or, one entire turn more than the human cochlea (Fig 2.1).

\subsection{Geometry of the Guinea Pig Scala Tympani}

This section will discuss the source for data on the scala tympani cavity of the guinea pig cochlea and also the methods employed for manipulating the data to a more agreeable form.

\subsubsection{Voie-Spelman Three-Dimensional Cochlear Reconstruction}

Three-dimensional (3D) data of the guinea pig cochlea was acquired from the research of Dr. Arne Voie and Dr. Francis Spelman of the University of Washington. In that work, they developed a method termed Orthogonal-Plane Fluorescence Optical Sectioning(OPFOS)and used this toobtaina3Dreconstructionofaguineapig cochlea [23].

The OPFOS method uses a laser and digital imaging system to gather spatial information on a specimen. An excised guinea pig cochlea is prepared by decalcification, dehydration, and clearing by a solution. The cleared cochlea is then treated with a fluorescent dye solution. When a laser beam is passed through the dye treated cochlea, the imaging camera which is directed orthogonally to the beam records the fluorescing spots produced by the interaction of the 

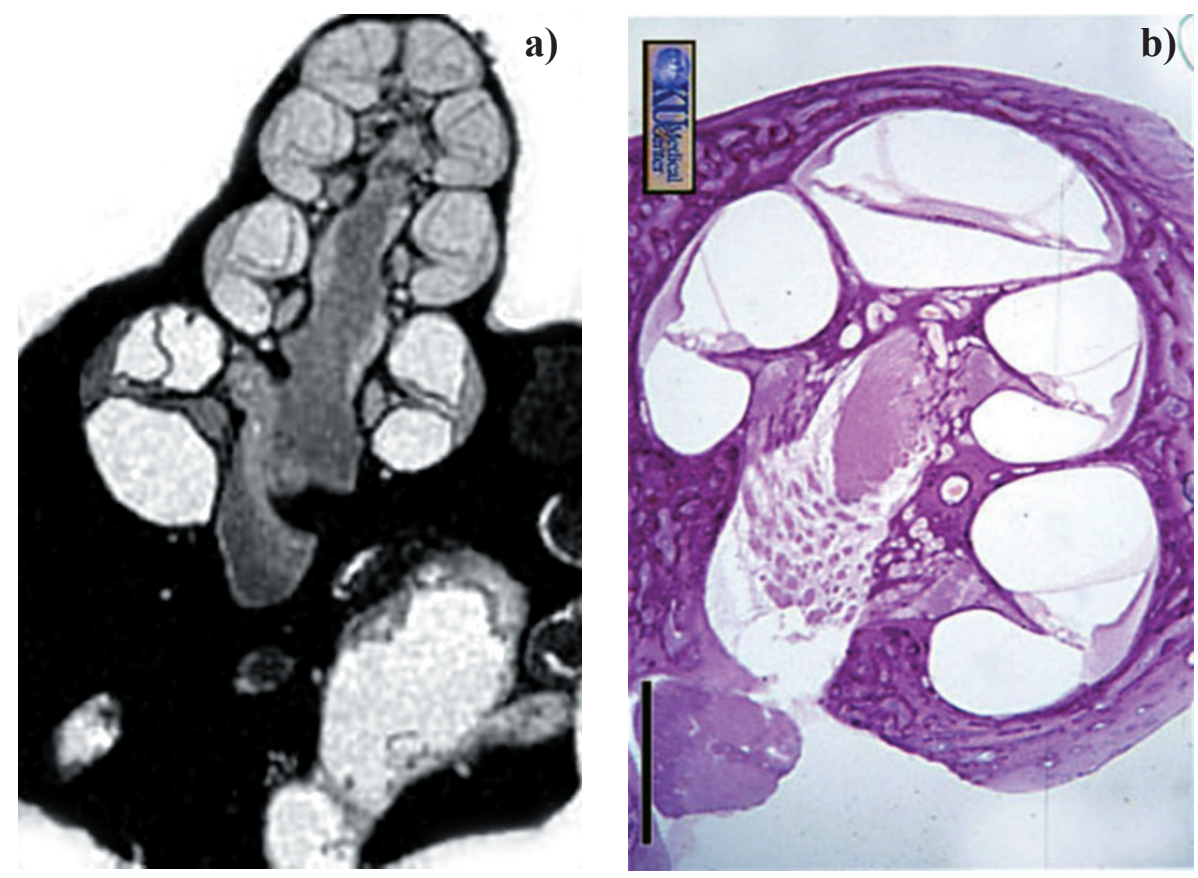

Figure 2.1 (a) Cross sections of a guinea pig[21] (b) and a human cochlea[22].
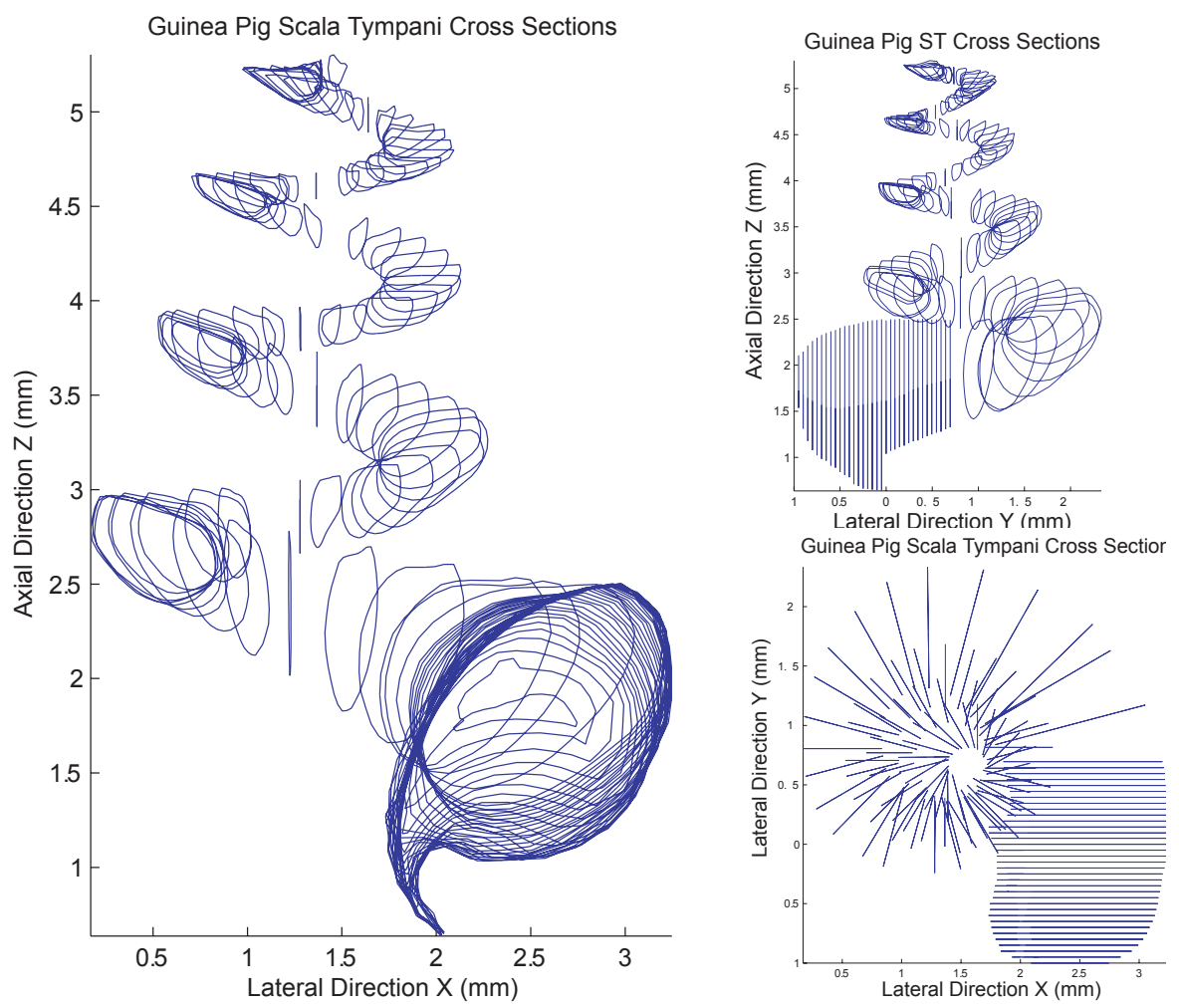

Figure 2.2 Voie-Spelman data of a guinea pig scala tympani. 
beam with the treated cochlear material. By translating the specimen under the beam, a series of two dimensional images (cross sections) can be combined using the stage translation information to construct a $3 \mathrm{D}$ image of the cochlea.

Using the OPFOS method and instrumentation, the authors report spatial resolutions of $10 \mu \mathrm{m}$ and $26 \mu \mathrm{m}$ in the lateral and axial directions, respectively. For the guinea pig scala tympani, 125 cross sections were recorded consisting of 3449 total points (Fig. 2.2).

\subsubsection{Parametric Formulation for the Guinea Pig Scala Tympani}

To aid in extracting pertinent spatial information for designing the actuatable implant, the Voie-Spelman data was used to develop parametric equations of key characteristics of the scala tympani. The parametric equations simplify the task of applying and mathematically manipulating the shape of the cochlea for operations such as machining programs and numerical modeling. The equations are based on $\theta$, the angular depth into the cochlea beginning at the basal end and extending to the apex. The origin for the angular insertion depth is located at the typical surgical insertion point of a cochlear electrode array, in this case the cochleostomy.

The most basal cross section for the Voie-Spelman data corresponds to the round window ledge. It was determined by direct measurement on a guinea pig cochlea that the center of the cochleostomy lies approximately $1.2 \mathrm{~mm}$ from the round window ledge (Fig. 2.3) [24]. This corresponds to the most basal 30 cross sections of the Voie-Spelman data. Since the insertion of the electrode array applies only to the apical cross sections, the 30 basal sections were not included in the formulation of the geometric equations.

A program was written in MATLAB to perform the calculations and data 


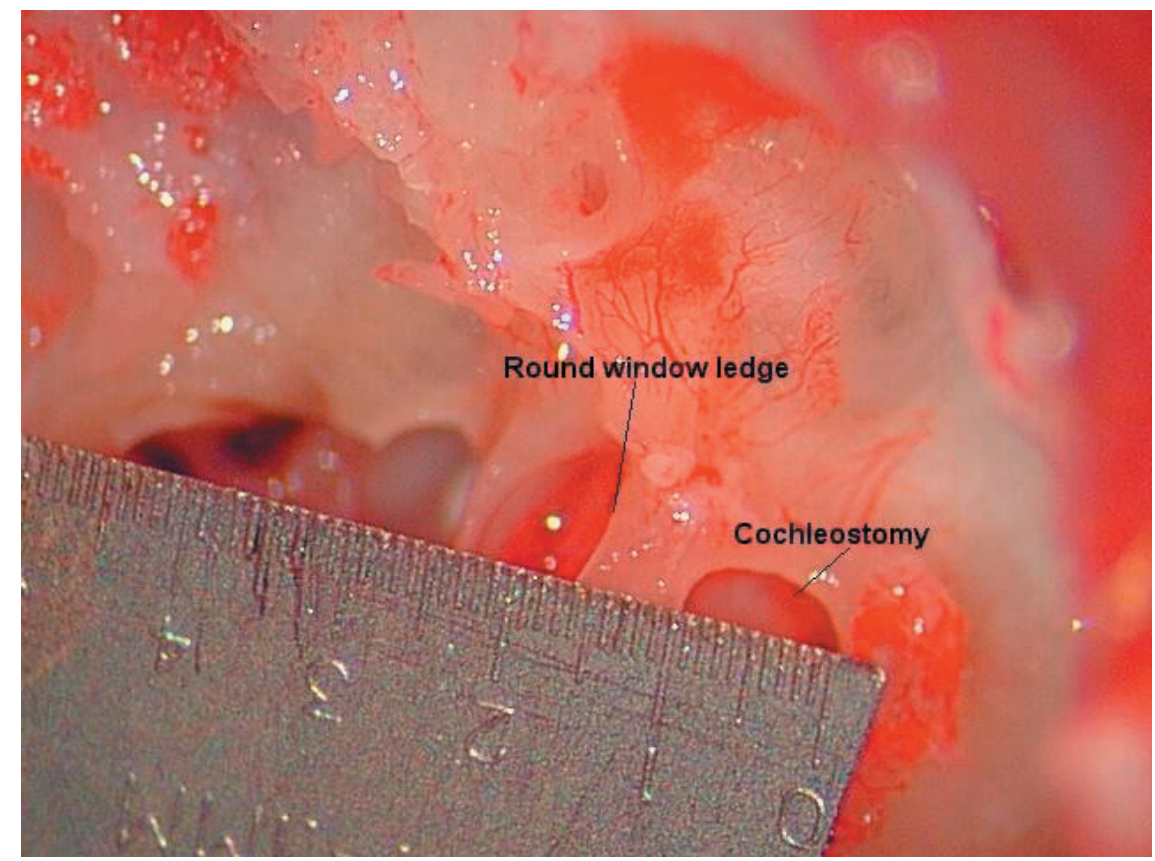

Figure 2.3 Direct measurement of the cochleostomy position.

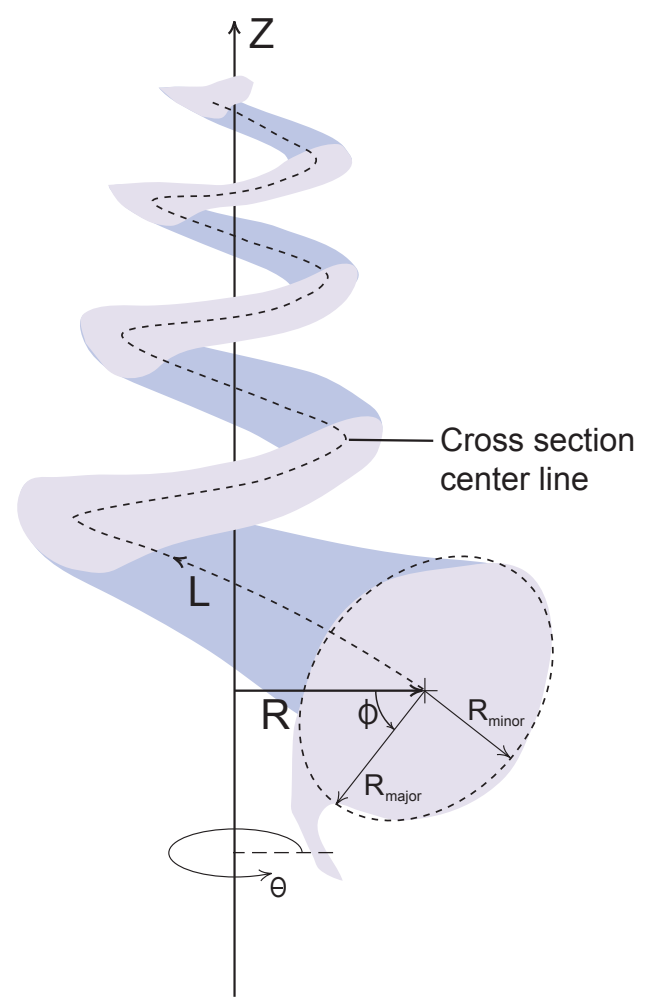

Figure 2.4 Coordinate system for ST mathematical model. 
manipulation required (see Appendix A). The data was first translated and rotated to align the $\mathrm{z}$ axis of the coordinate system with the helical axis (modiolar axis) of the ST data using basic transformation matrix manipulations commonly employed in 3D CAD software [25]. The resulting translations and rotations are outlined in Table 2.1.

The first attribute of the geometry data to be described is the position of the scala tympani (ST) centerline that runs through the average position of each cross section. The coordinate system is changed from rectangular to cylindrical as this is more appropriate considering the helical nature of the ST. By basic trigonometry, the angle $(\theta)$ of each cross section center relative to the first section and about the z-axis was determined. This established the total angular depth into the cochlea as $1358^{\circ}$. Next, the orthogonal radius was found between the section center points and the z-axis (Fig. 2.4). The z-coordinate remains unchanged in the conversion since it corresponds to the z-axis of the cylindrical coordinate system. Using a least squares curve fitting algorithm, polynomials were then fit to the radius and z-height to formulate the equations based on the $\theta$ parameter (Table 2.2).

An additional useful parametric equation that was defined describes the length of the ST centerline. The arc lengths between the center points of each adjacent cross section was found by calculating the straight line chord distance between the center points and using the average of the

\begin{tabular}{|c|c|}
\hline Table 2.1 & Translations and Rotations of the ST Data \\
\hline X Translation & $1.00 \mathrm{~mm}$ \\
\hline Y Translation & $-1.25 \mathrm{~mm}$ \\
\hline Z Translation & $-2.00 \mathrm{~mm}$ \\
\hline X Axis Rotation & $6.30^{\circ}$ \\
\hline Y Axis Rotation & $5.73^{\circ}$ \\
\hline
\end{tabular}




\begin{tabular}{|c|c|c|c|}
\hline \multicolumn{5}{|c|}{} \\
Table 2.2 Parametric Equations for the Geometry of a Guinea Pig ST \\
\hline $\begin{array}{c}\text { ST Centerline } \\
\text { Attribute }\end{array}$ & $\begin{array}{c}\text { Polynomial Expression }(\theta \text { in degrees) } \\
\text { Radius }\end{array}$ & unit & $\begin{array}{c}\text { RMS } \\
\text { error }\end{array}$ \\
\hline Z Height & $\begin{array}{c}\mathrm{R}=2.296 \mathrm{e}-12 \theta^{4}-7.707 \mathrm{e}-9 \theta^{3}+8.745 \mathrm{e}-6 \theta^{2} \\
-4.215 \mathrm{e}-3 \theta+1.351\end{array}$ & $\mathrm{~mm}$ & 0.049 \\
\hline Length & $\begin{array}{c}\mathrm{Z}=6.464 \mathrm{e}-13 \theta^{4}-1.797 \mathrm{e}-9 \theta^{3}+8.012 \mathrm{e}-7 \theta^{2} \\
+3.016 \mathrm{e}-3 \theta-3.790 \mathrm{e}-2\end{array}$ & $\mathrm{~mm}$ & 0.028 \\
\hline $\begin{array}{c}\text { ST Cross Section } \\
\text { Attribute }\end{array}$ & $\begin{array}{c}\mathrm{L}=-4.012 \mathrm{e}-12 \theta^{4}+1.188 \mathrm{e}-8 \theta^{3} \\
-1.537 \mathrm{e}-5 \theta^{2}+1.969 \mathrm{e}-2 \theta\end{array}$ & $\mathrm{mm}$ & 0.039 \\
\hline Major Radius & $\begin{array}{c}\mathrm{R}_{\text {major }}=9.403 \mathrm{e}-13 \theta^{4}-3.195 \mathrm{e}-9 \theta^{3}+4.023 \mathrm{e}-6 \theta^{2} \\
-2.279 \mathrm{e}-3 \theta+0.711\end{array}$ & $\mathrm{~mm}$ & 0.017 \\
\hline Minor Radius & $\mathrm{R}_{\text {minor }}=8.526 \mathrm{e}-13 \theta^{4}-2.752 \mathrm{e}-9 \theta^{3}+3.314 \mathrm{e}-6 \theta^{2}$ \\
$-1.860 \mathrm{e}-3 \theta+0.529$ & $\mathrm{~mm}$ & 0.016 \\
\hline Tilt & $\phi=1.009 \mathrm{e}-12 \theta^{4}-2.343 \mathrm{e}-9 \theta^{3}+1.780 \mathrm{e}-6 \theta^{2}$ & $\mathrm{deg}$ & 0.043 \\
\hline
\end{tabular}

radii for each of the two points with the corresponding included angle.

With the path of the ST defined, it is necessary to describe the shape and size of the space along that path. The complicated cross-sectional shape of the ST is simplified by fitting each cross section with an ellipse. The ellipse fitting method finds values for the major and minor radii as well as estimating the degrees of in-plane rotation of the ellipse around its center point. Again, a curve fitting algorithm was used to define these attributes against the angular depth parameter, $\theta$ (Table 2.2). The tilt is read as positive in the counterclockwise direction along the centerline path towards the apex.

Figure 2.5 shows the scala tympani as reconstructed using the parametric equations to build a 3D graph using a program written in MATLAB (Appendix A). As can be seen from the figure, the mathematical model gives a well behaved approximation to the guinea pig ST geometry. 
a)

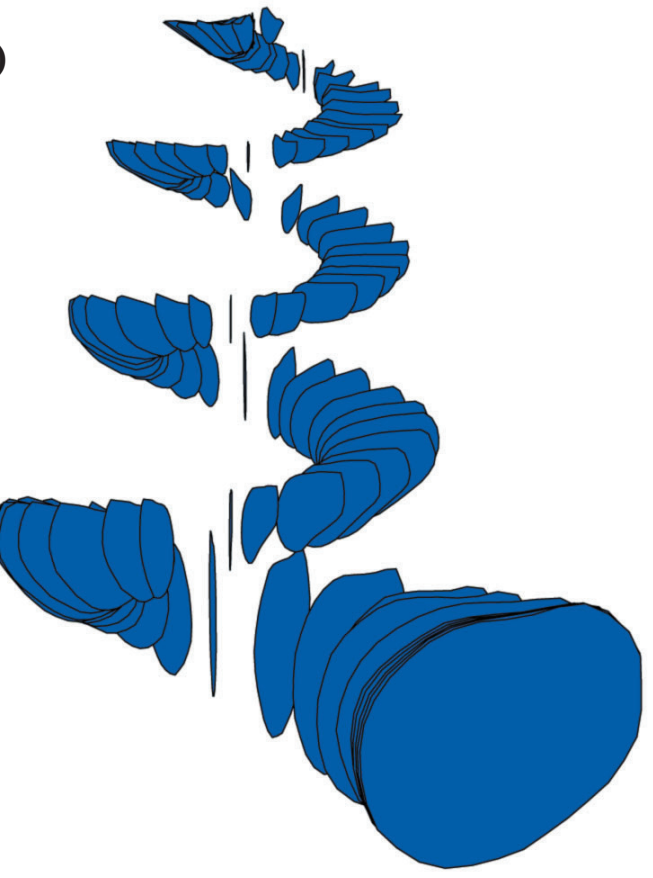



Figure 2.5 (a) Cross Sections of the ST (b) ST reconstructed from the parametric equations.

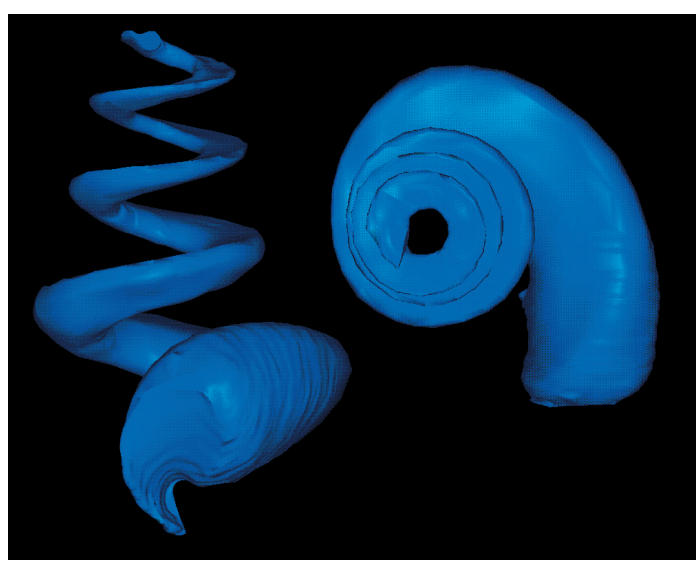

Figure 2.6 IDEAS model of the ST.



Figure 2.7 3x acrylic ST replica. 


\subsubsection{Guinea Pig ST Insertion Testing Cavities}

It became clear with early prototypes of the insertion tool that a replica of the ST cavity would be helpful in testing of performance. Criteria for the ST replicas included using a clear material so that observation of the insertion would be possible and it would be constructed at several scales since early prototypes were made at a larger scale.

The first replicas at the $3 \mathrm{x}$ and $10 \mathrm{x}$ scales were manufactured with the aid of rapid prototyping. Rapid prototyping allowed for the complex shape of the ST to be quickly duplicated in a hard wax material. A solid model of the ST was created in IDEAS modeling software using the Voie-Spelman cross section information. The point coordinates were imported into the software and then lofted and smoothed between the sections to make the computer model (Figure 2.6).

This model was then converted into a file suitable for the rapid prototyping machine at the $1 \mathrm{x}, 3 \mathrm{x}$ and $10 \mathrm{x}$ scales. The particular rapid prototyping technique employed uses an extruded wax filament from the tip to deposit beads of material in the $x-y$ plane. The controlled extrusion forms one layer as the part platform is lowered to build one cross section of the three-dimensional reproduction at a time. It is by this method that the solid wax replicas were produced.

The wax replicas were then suspended in rectangular cavities and a two part clear acrylic epoxy was poured around them. After the acrylic was cured, the replicas were removed from the mold and placed in a bath of mineral spirits heated on a hotplate under a fume hood at approximately $110^{\circ} \mathrm{C}$. The solvent bath was used to dissolve out the prototyping wax. The result was a translucent cavity with the shape of the guinea pig ST (Figure 2.7). The cavity is translucent and not clear due to the rough inner surface left by the prototype wax replica. 
For the $3 \mathrm{x}$ and $10 \mathrm{x}$ sizes, the ST cavities fabricated with this method worked very well. However, for the $1 \mathrm{x}$ insertion cavity the rapid prototype machine lacked the necessary resolution to create the wax model. Therefore, it was necessary to build the scale cavity using an alternate method.

For the $1 \mathrm{x}$ ST cavity, a Dover precision micromilling machine was used to machine a cavity into a $2 \mathrm{~mm}$ thick acrylic sheet. A $250 \mu \mathrm{m}$ diameter ball end mill was used in several passes to make approximately the first $340^{\circ}$ of the ST (Fig. 2.8). Since the successive turns of the ST overlap one another, more than one turn would be very difficult to fabricate using machining methods.

The machining program used coordinates provided by the parametric equations discussed earlier to make a semi-elliptical shape (Appendix B). The tool was moved helically and stepped laterally (30 steps) to mill the model. A second mirror image was also made so that the two halves could be combined to form a

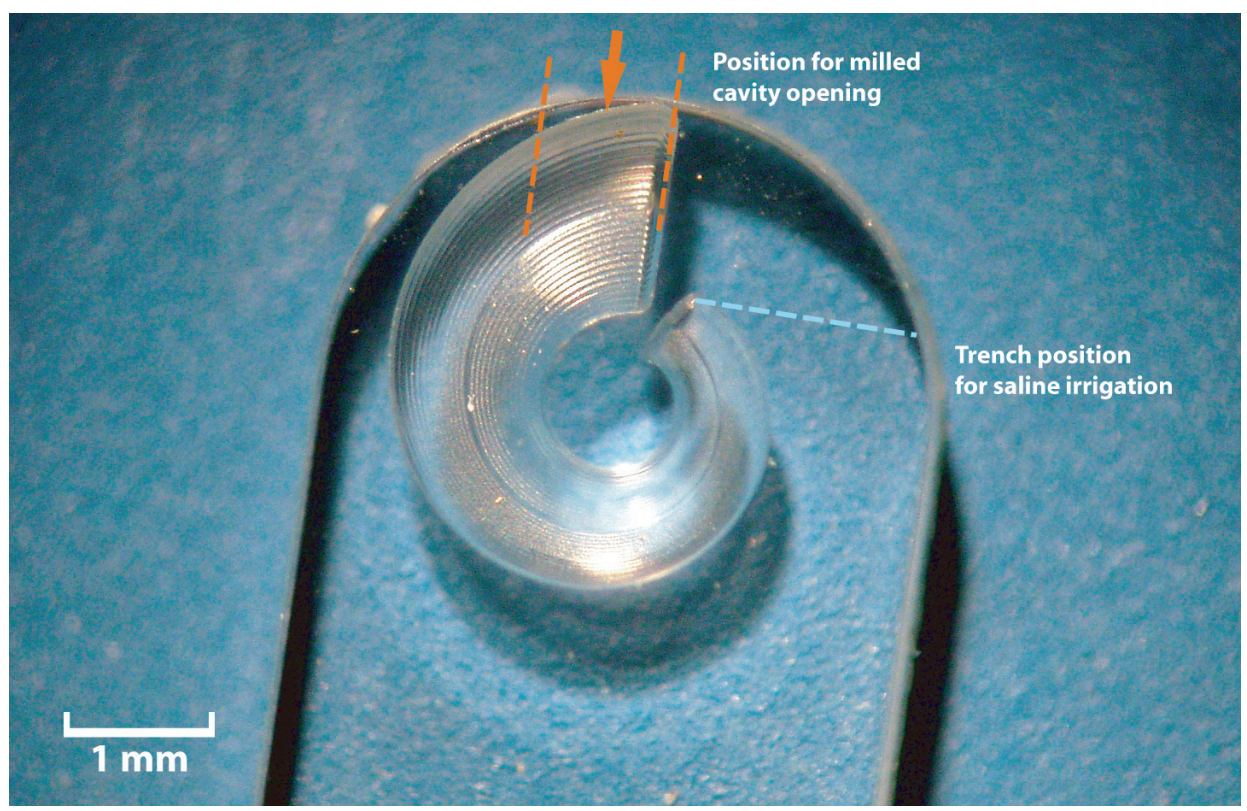

Figure 2.8 Machined 1x scale single-turn ST insertion cavity. 
complete cavity. The pieces were removed from the sheet using a $2 \mathrm{~mm}$ end mill. The curved portion provides a thin shell into which a "cochleostomy" opening can be made while the straight portion provides for convenient handling. The two halves were combined using a thin layer of UV cure adhesive. The opening into the cavity was made using a $1 \mathrm{~mm}$ milling tool bored directly into the end of the rounded end.

For several of the models fabricated, a $225 \times 225 \mu \mathrm{m}$ trench was machined from the apical end of the cavity to the outside of the model and a $200 \mu \mathrm{m}$ outer diameter steel hypodermic tube was affixed into it using UV cure adhesive. A 1/8th inch nylon hose barb was epoxied over the exterior portion of the hypo. This provides a port to which rubber tubing could be attached that would supply saline solution into the cavity. The saline solution would fill the cavity making the conditions closer to that of an in-vivo guinea pig insertion. 


\subsection{Fluidically Actuated Cochlear Implant Design}

The design of the fluidically actuated cochlear implant tool includes

one or more polymer chambers with fluidic connections to a pressure source. The chambers are designed to be integrated to a silicon electrode array being designed concurrently with the surgical tool at the University of Michigan, Ann Arbor. The electrodes arrays will eventually incorporate sensors along the length and a computer processor at the proximal end (back end).

\subsection{Silicon Electrode Array}

Currently, a cochlear electrode array is being developed using thin-film technology at the University of Michigan, Ann Arbor as part of the Engineering Research Center for Wireless Integrated Microsystems. These monolithic implants may eventually provide human patients with the advantages over the traditional wire bundle cochlear implants. The thin flexible arrays may allow greater insertion precision and depth. Also, the electrodes can be spaced closely together to provide a greater number of electrodes with better localization of the stimulation.

The insertion tool developed in this project was designed for inserting this type of cochlear electrode array into a guinea pig scala tympani. The design is flexible in that it can be modified to suit the insertion of other types of cochlear implants or thin electrodes.

\subsubsection{Electrode Array (Probe) Design}

The electrode array is fabricated with bond pads, interconnects, and stimulating site materials deposited on top of a silicon substrate. The silicon substrate is thin and extremely flexible; however, it is also fragile and may fracture if excessive tensile stress or contact force is placed on it. Its flexibility 

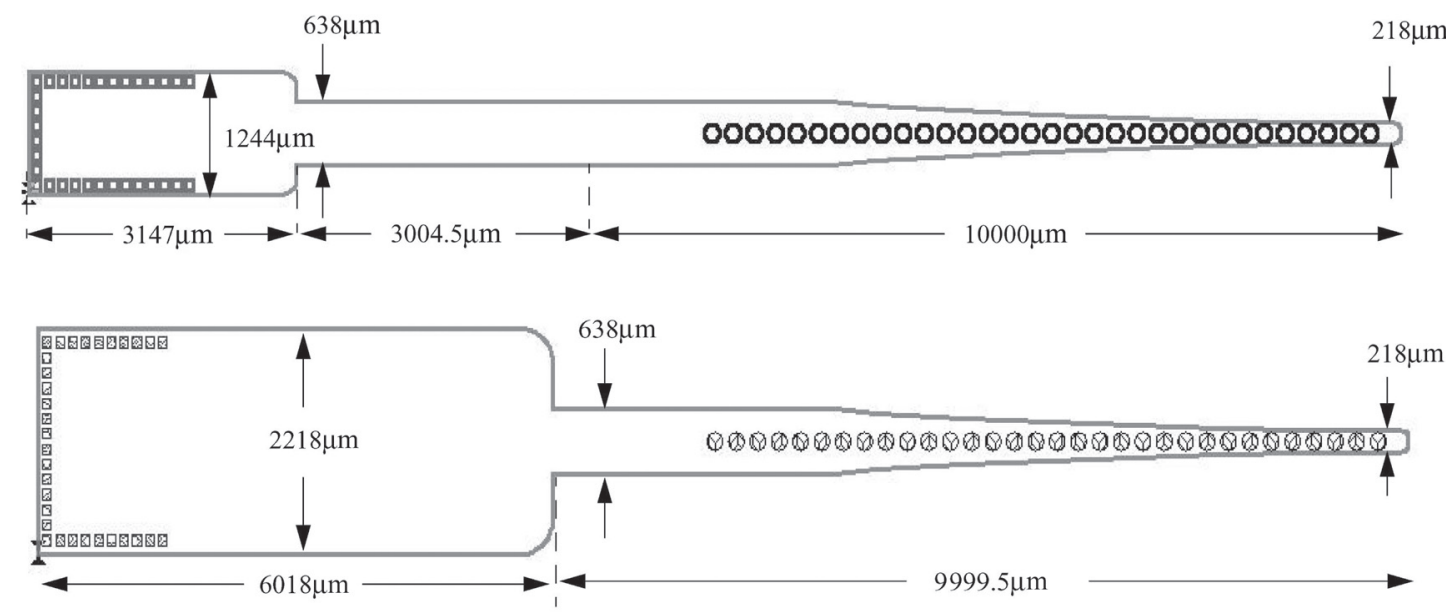

Figure 3.1 Layouts for the high density silicon electrode arrays [26].

is also limited to the plane of bending orthogonal to its thickness direction.

The initial design will be compatible with the guinea pig ST to allow invivo testing of the electrode array. The dimensions for the electrode array were defined by the parametric model of the ST discussed earlier. The details of the array layout are given in Figure 3.1. The layout with the large back end will eventually incorporate the circuitry for an implantable microcontroller. The small back end layout allows for a greater number of probes to be fabricated on each wafer during the development stage and also has a longer shaft region for insertion tests.

The probe currently features $32 \mathrm{IrOx}$ (iridium oxide) stimulating sites, each with a diameter of $150 \mu \mathrm{m}$ on a $250 \mu \mathrm{m}$ pitch, and will eventually incorporate 128 sites. The inserted portion of the array has a $4 \mu \mathrm{m}$ thick substrate while the larger thicker $(14 \mu \mathrm{m})$ portion remains outside of the cochlea to support electrical connections and circuitry.

The array tapers from a width of $200 \mu \mathrm{m}$ at the tip (apical end) to $638 \mu \mathrm{m}$ at the basal end over a $10 \mathrm{~mm}$ length. Since the guinea pig cochlea has an additional 


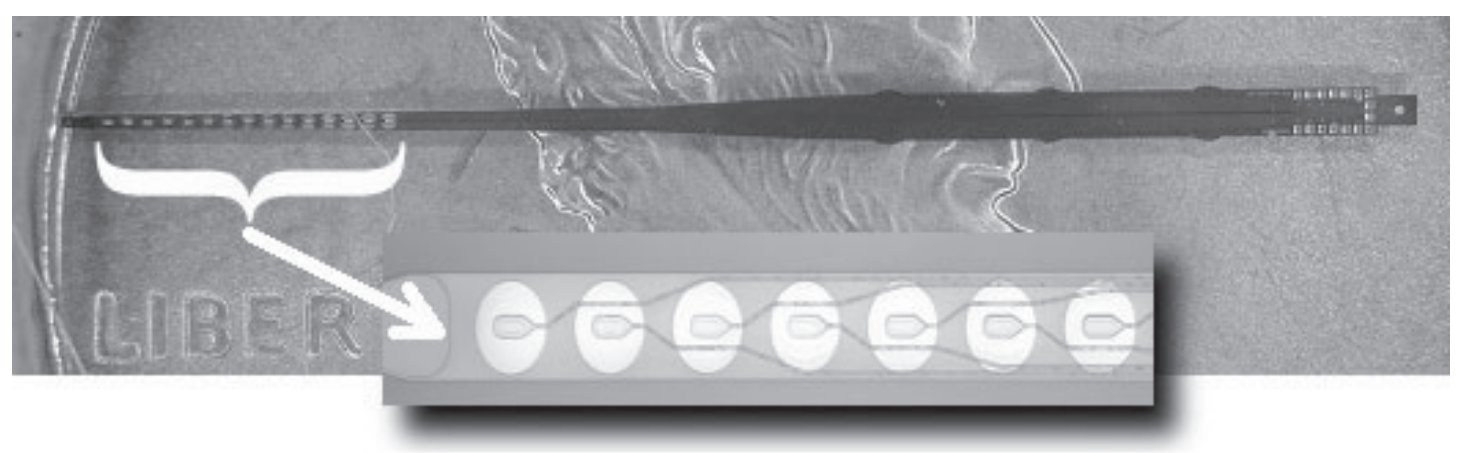

Figure 3.2 High-density monolithic cochlear electrode array [26].

spiral turn when compared to the human cochlea, research has focused on developing the tool for the first $10 \mathrm{~mm}$ of the insertion depth (this length encompasses the same number of turns as the human cochlea). Within the scala tympani, the array assumes a position where the electrodes will face inwards toward the modiolus so that the normal of each stimulating site is perpendicular to the cochlea's spiral axis. Figure 3.2 illustrates the high-density cochlear electrode array.

\section{Fabrication of the Silicon Electrode Array}

Fabrication of the passive electrode arrays begins with two boron etchstop diffusions to define the substrate. The thinner $(4 \mu \mathrm{m})$ portion of the substrate will be inserted into the cochlea, while the thicker $(14 \mu \mathrm{m})$ portion remains outside to support electrical connections to the array (and eventual circuitry). A stress-compensated dielectric stack of silicon dioxide, silicon nitride, and $\mathrm{IrOx}$ $(2000 \AA / 1000 \AA / 1000 \AA)$ is next deposited by low-pressure chemical vapor deposition

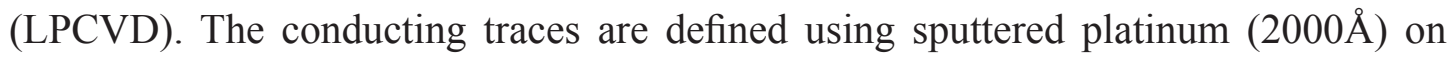

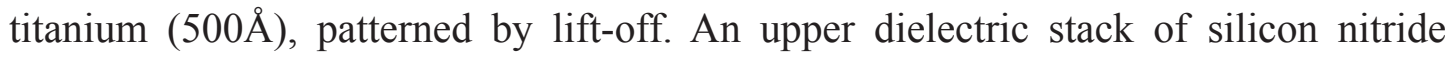
and a low temperature oxide $(5000 \AA / 15,000 \AA)$ is deposited by plasma-enhanced chemical vapor deposition to passivate the interconnect. Iridium (3000§) over titanium $(500 \AA)$ is sputtered and patterned by lift-off to define the stimulating 
sites. Gold bonding pads are formed on the back-end in a similar manner. Eventually, a parylene overcoat will be applied over the entire array and backing and will be selectively removed from the stimulating sites using laser ablation [26].

\subsubsection{Probe Level Sensors}

In addition to the IrOx electrodes on the array, position and touch sensing elements are being developed to provide feedback on the shape and location of the probe within the cochlea. Typically, the insertion of implants is done with little to no information on the status of the implant within the cochlea besides what can be felt by the surgeon's hand. Depth of insertion is measured by the length of the implant remaining outside the cochlea. More detailed information on position can be obtained post-operatively by medical imaging techniques, but an additional invasive procedure would be needed to make adjustments to the implant position.

With the addition of strain and touch sensors to the implant, invaluable insight can be gained about the insertion procedure in real time. Strain gages on the flat face along the length of the implant can estimate the curvature of the probe and compare that against the geometric model to estimate depth and position of the probe within the cochlea. Touch sensors along the edge of the probe and at the tip provide information on possible obstructions and alert against hard contact points that could lead to trauma.

The strain and touch sensors are being developed at the University of Michigan, Ann Arbor in a process that will eventually be merged with the process used to fabricate the electrodes on the silicon implant. The substrate contains eight segmented piezoresistive strain gauges, each having a resistance of about $100 \mathrm{k} \Omega$ and a gauge factor of 20 (Fig 3.3). Figure 3.4 illustrates the lateral

touch sensors in several probe designs. Deformation of the "lace" induces an 


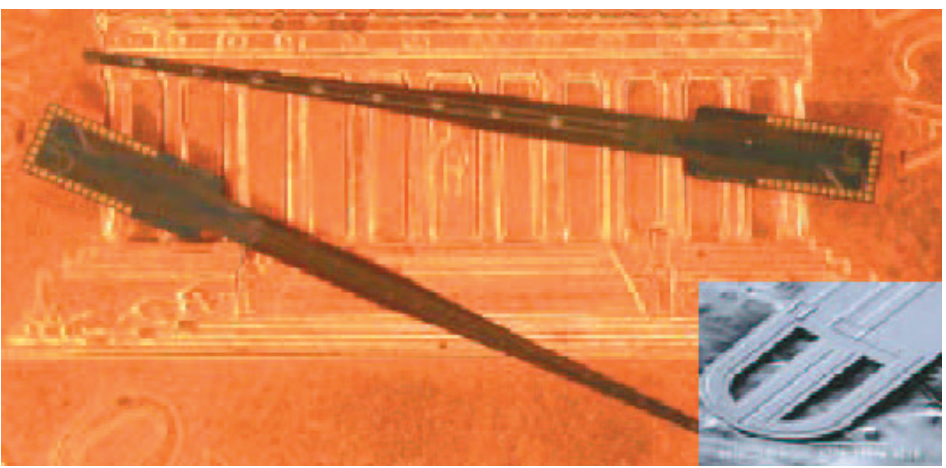

Figure 3.3 Probe level peizoresistive strain gages \& tip sensor (inset) [27].


Figure 3.4 Probe level lace lateral touch sensors [27].

electrical response. Through measurement of the strain gages, array shape can be determined using the curvature sensor outputs to better than $50 \mu \mathrm{m}$ [27].

\subsection{Articulated Electrode Array Backing Tool}

The insertion tool is intended to achieve a low-resistance basal insertion of the electrode array to attain the deep perimodiolar positioning required for auditory nerve stimulation while minimizing intracochlear trauma. The device consists of multiple fluidic chambers, providing localized curvature 
control along the length of the attached silicon electrode array, similar to the pneumatic response of a Bourdon tube common to mechanical pressure gages.

The device at its rest state will have the spiral shape of the guinea pig scala tympani for an optimal modiolus-hugging configuration. The chambers are molded in a tapered-helical configuration to correspond to the ST shape after relaxation of the chambers. This ensures the optimal electrode placement within the cochlea to be retained after the backing device becomes dormant (post-operative). The insertion device is bonded to the electrode using medical grade adhesive. Actuation is achieved by injecting fluid into the chambers and regulating the fluidic pressure to change the curvature. In the current design, the positioning device remains within the cochlea with the electrode array after insertion since it permanently bonded to the electrode array. After insertion, the pressure inlets to the backing device would be hermetically sealed to isolate the internal spaces of the actuation chamber(s).

The chambers are arranged in a stacked overlapping design (Fig. 3.5) to provide fluidic connections to a pressure controller and to allow for local curvature control of the device. The stacked tube configuration allows for localized curvature control of various areas along the length of the electrode and additional stiffening and actuating ability towards the base. The actuating chambers are bonded to the silicon electrode array using a medical grade UV-cure adhesive. The spiral, helical shape of the scala tympani is then impressed on these stacked chambers. Curvature is effected along the entire length of the chambers. In sections of multiple chambers, the resulting actuation is cumulative as each chamber is pressurized.

Actuation of the fluidic chambers is required to adjust the bending curvature about the electrode face of the probe. To accomplish this, the actuation chambers are mounted 


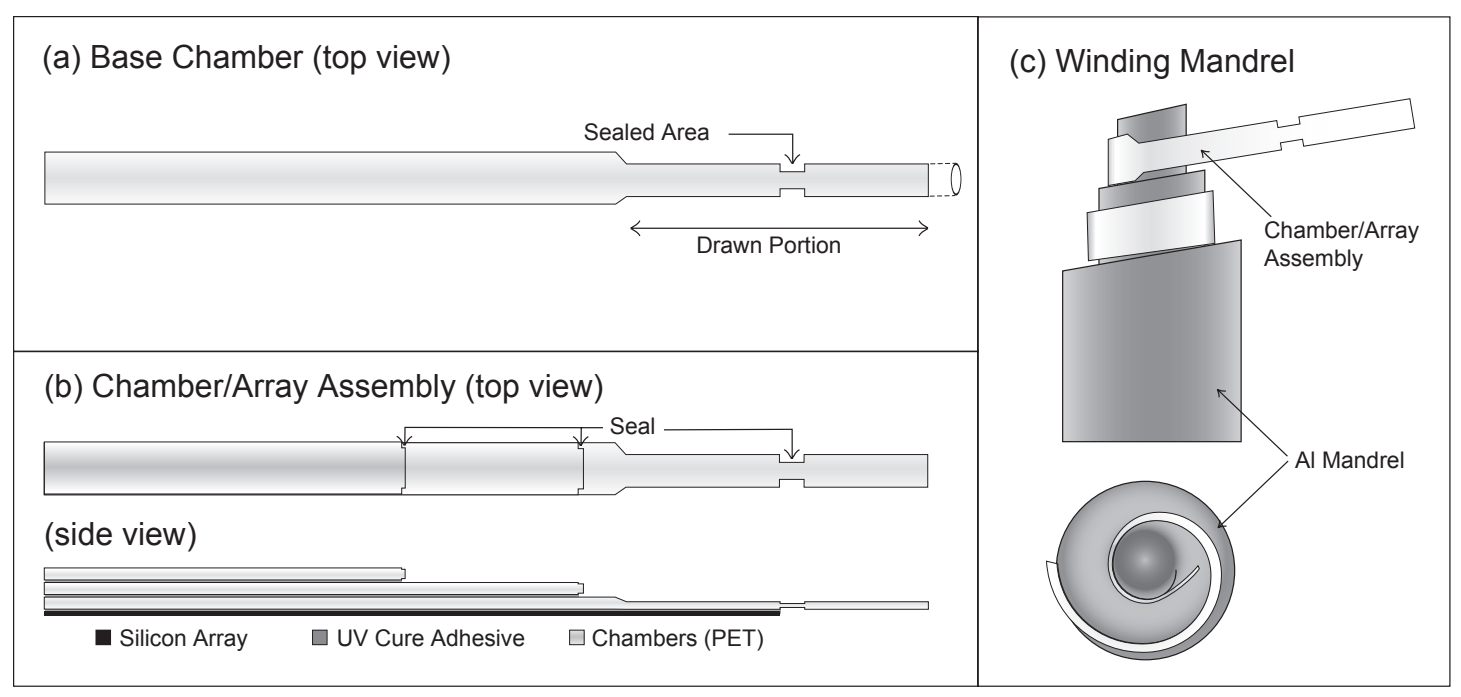

\section{Figure 3.5 Basic manufacturing steps and arrangement for the overlapping chamber design of the insertion device.}

to the backside of the probe (the face opposite the electrodes) and along the entire length. The base chamber that mounts directly to the probe runs the entire $10 \mathrm{~mm}$ length.

For additional localized control of curvature, additional chambers are stacked onto the first. These stacked chambers run from the basal end to some length short of the chamber upon which it is mounted. In this way, the base chamber provides actuation for the entire length of the device while the additional chambers stacked on top provide for more localized actuation as the insertion proceeds.

Elastic springback in the material provides the necessary force to return the device back to the original perimodiolar shape after the insertion procedure is complete and fluid pressure is removed. Thus, the insertion device will provide the necessary shape for the electrode array to fit the cochlea during insertion, thereby eliminating most friction forces that prevent deep insertion. Eventually, a parylene overcoat will be applied over the entire assembly to seal and protect the device and specific areas 
of the coating will be removed from the IrOx stimulating sites using laser ablation.

\subsection{Actuation Principle of the Fluidic Chambers}

The actuation principle is similar to that of Bourdon tubes commonly found in dial pressure gauges (Fig 3.6). In these types of gauges, a coiled tube with a non-round cross section(CS) is pressurized causing the cross section to elastically deform towards a more rounded shape. As the cross section changes, the radius of the coiled tube increases (the primary dimensions and configuration of a Bourdon tube element are summarized in Figure 3.7). The deflection of the free end as the radius increases moves the gauge pointer via a series of linkages allowing the pressure inside the tube to be read on the gauge face. The application of this type of actuation to a cochlear implant tool follows the same principles but the tube elements are highly elastic and the deflections are much larger.

Despite the fact that Bourdon tube gages have been in use for over 100 years, little literature is available on an analytical solution to the actuation principle. Gauge manufacturers use old standards based on past designs and estimate new designs based off of these.

A publication by Alfred Wolf in 1946 presented a theory based on two elements of strain, one in the transverse section of the tubing and one in the longitudinal direction parallel to the tubing axis [28]. However, he concluded that the theory of pure bending in thin shells did not adequately account for the case of $a^{4} / R^{2} b^{2} \quad$ significantly greater than unity, which is the case for the

dimensions required in the cochlear implant backing (where $a^{4} / R^{2} b^{2}>25$ ).

In a Master's Thesis, Cynthia Conway [29] attempts an analytical 

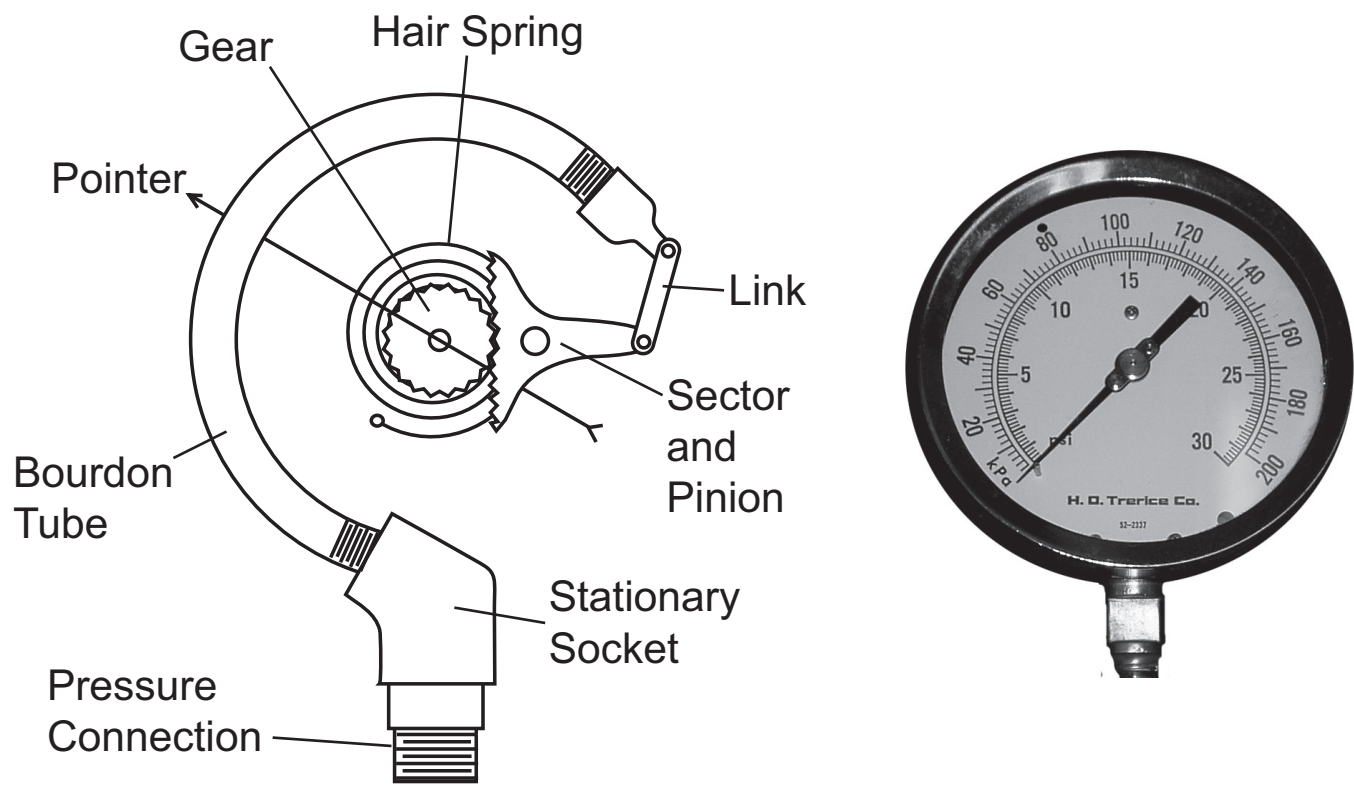

Figure 3.6 A Bourdon tube as applied to a C-type pressure gage.
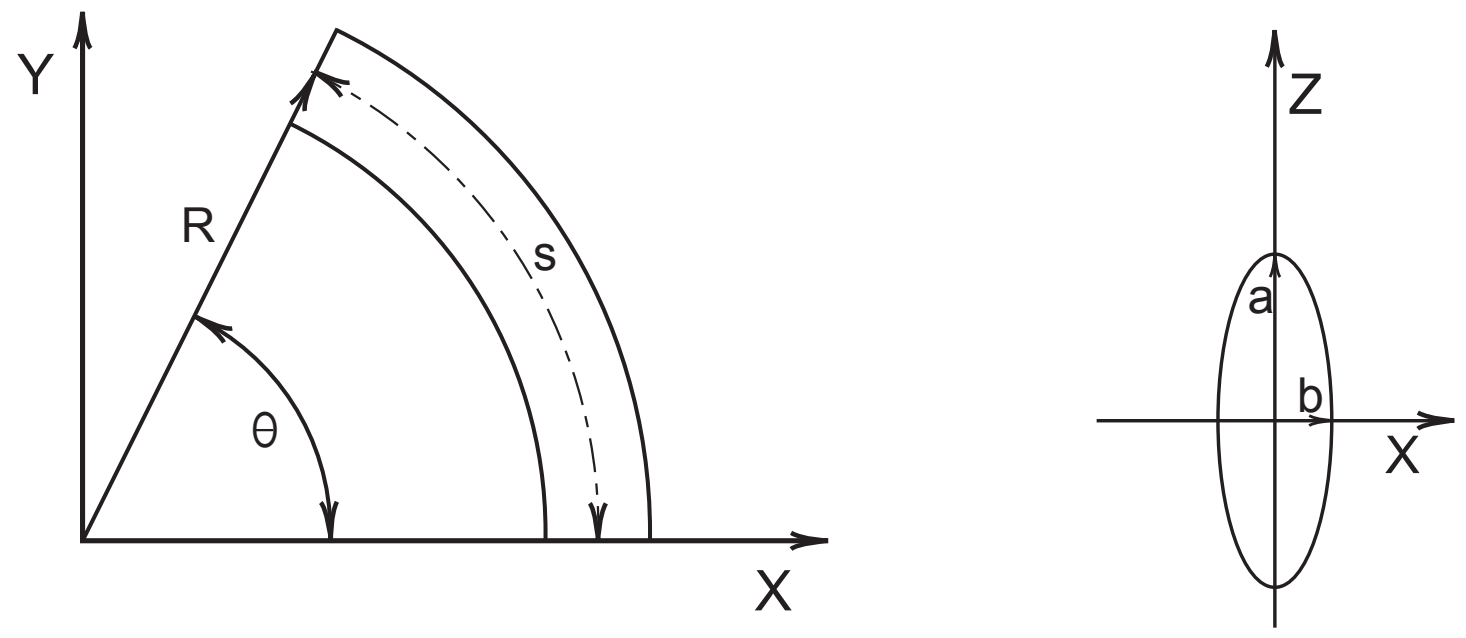

Figure 3.7 Variable definitions for Bourdon tube element and cross section geometry. 
solution to an elliptical cross section Bourdon tube. The formulation equates the sum of the change in strain energy in the cross section due to rounding and the change in strain with the bending of the tube to the differential increase in virtual work from pressurization. Tip deflection is calculated using Castigliano's second theorem and the solution is resolved in an iterative FORTRAN program.

The solutions produced in the Conway analytical model were compared to experimental data and to an FEA model of a Bourdon tube. The experimental data included measurements from several commercial gages and two tubes fabricated to the author's specifications. The FEA model was developed in I-DEAS using linear thin shelled elements. However, the analytical model was not verified by either experimentation or FEA results. In the author's own words: "the validity of the analytical model remains under investigation". Furthermore, the analytical model is highly sensitive to the geometries of the tube. Solutions for cross section ratios of a:b outside of 2:1 yielded no result. For the cochlear implant backing, cross section ratios range above 5:1, far beyond the scope of the analytic model.

Owing to the lack of a published modeling technique suitable for the Bourdon-type actuation, it therefore became necessary to formulate a method for estimating actuation to aid in design of the implant tool. The theory presented here is a combination of observations from actuation experiments, mechanics theories and models and geometric constraints.

\subsubsection{Experimental Results of Fluidic Chamber Actuation}

Radial Actuation of Chambers with Pressurization

Several experiments were carried out to study the actuation potential of the fluidic chambers and to gain insight that could lead to a model of the 
actuation mechanics to aid in the design process of the cochlear implant backing. The first experiments explored the relationship of the overall radius of a semicircular chamber (approximately $180^{\circ}$ ) at different internal pressures.

PET tubing with a $200 \mu \mathrm{m}(0.008$ ') inner diameter (ID) and a $4 \mu \mathrm{m}(0.00015 ")$ thick wall was flattened to an elliptical cross section, formed into a semicircular, constantradius actuation chamber using the methods outlined in Chapter 4. The radius of the chambers varied from $1 \mathrm{~mm}$ up to $10 \mathrm{~mm}$. The chamber was then attached to the in-house pneumatic air lines through a diaphragm type pressure regulator. A large-faced pressure gage with a range of $0-35$ psi was used to read the internal pressure of the chamber.

The chamber was mounted under an Olympus stereoscopic microscope with the axis of the radius parallel with the viewing point of the optics. Images of the actuation were taken with a digital camera and the focus and magnification of the microscope remained untouched throughout the experiment. To improve imaging of the clear PET chamber, the upper edge (closest to the optics) was colored lightly with ink.

Pressure was increased slowly to each target pressure and a picture was taken through the microscope with the digital camera. The pressure was then released to observe the springback of the device which was also recorded with the camera. A vacuum was then applied using a vacuum pump to return the tube to its original curvature and the results recorded. Typical photographs from the experiment are shown in Figure 3.8.

The radius of the chamber is measured using a program written in MATLAB (Appendix C). The program imports the digital photographs of the actuation and the mouse is used to record coordinates along the arc of the chamber in the picture. The program then calculates the radius of the chamber by comparing the 


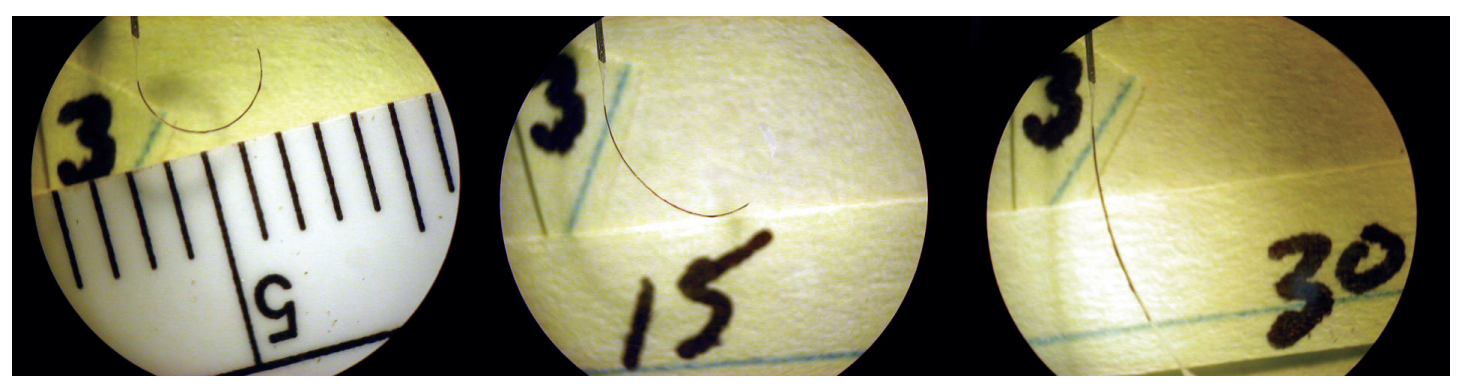

Figure 3.8 Chamber actuation at 0,15 and 30 psi (scale in $\mathrm{mm}$ ).

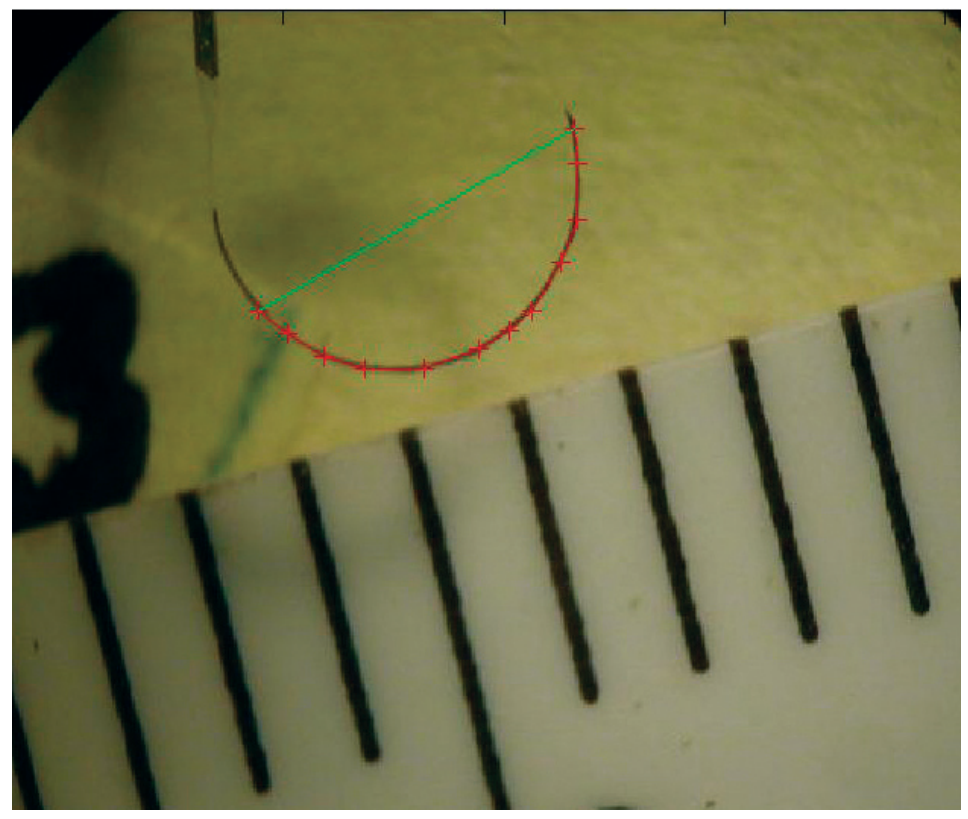

Figure 3.9 Measuring chamber radius. 
arc length and chord length as determined from the coordinates (Fig. 3.9). The scale was determined from the ruler marks recorded in the first photograph. The technique when used on the chamber photograph of zero pressure yielded a result of $\mathrm{R}=9.54 \mathrm{~mm}$, within $5 \%$ of the intended manufactured curvature of $10 \mathrm{~mm}$.

The measurements of the chamber radii using the program significant variation, especially at high pressures when the chamber shape approaches an almost straight configuration. The standard deviation $\left(s^{2}\right)$ of radius measurement results for chambers with a manufactured radius of $1 \mathrm{~mm}$ at $0 \mathrm{psi}$ inflation pressure was $0.0125 \mathrm{~mm}$ and $0.9441 \mathrm{~mm}$ at $30 \mathrm{psi}$. This measurement variation is the result of a combination of inherent user input variation as the arc points are picked on the photograph using the mouse and the sensitivity of the geometric calculation. As the radius increases, the ratio of arc length to chord length approaches unity and acts as a scalar to the input variation. To reduce measurement variation, each measurement was performed five times and then averaged to statistically reduce measurement variation to $0.0025 \mathrm{~mm}$ and $0.1888 \mathrm{~mm}$ at 0 and $30 \mathrm{psi}$ respectively $\left(s_{\text {avg }}^{2}=s^{2} / n\right)$.

The results of the experiment for six chambers manufactured on a $0.99 \mathrm{~mm}$ radius mandrel (as measured with a digital micrometer) is illustrated in Figure 3.10. Several often observed characteristics of chamber actuation are evident in this sample set. Chambers 2 through 5 are what would be considered a "normal actuation" consisting of a slow initial increase of radius with pressure that progressively begins to increase more with increased pressure. Differences between the measurements among these samples are likely due to slight variations in manufactured geometry.

Chamber 1, on the other hand, has a significantly greater increase of radius with pressure compared with chambers 2 through 5. Conversely, chamber 6 has a 


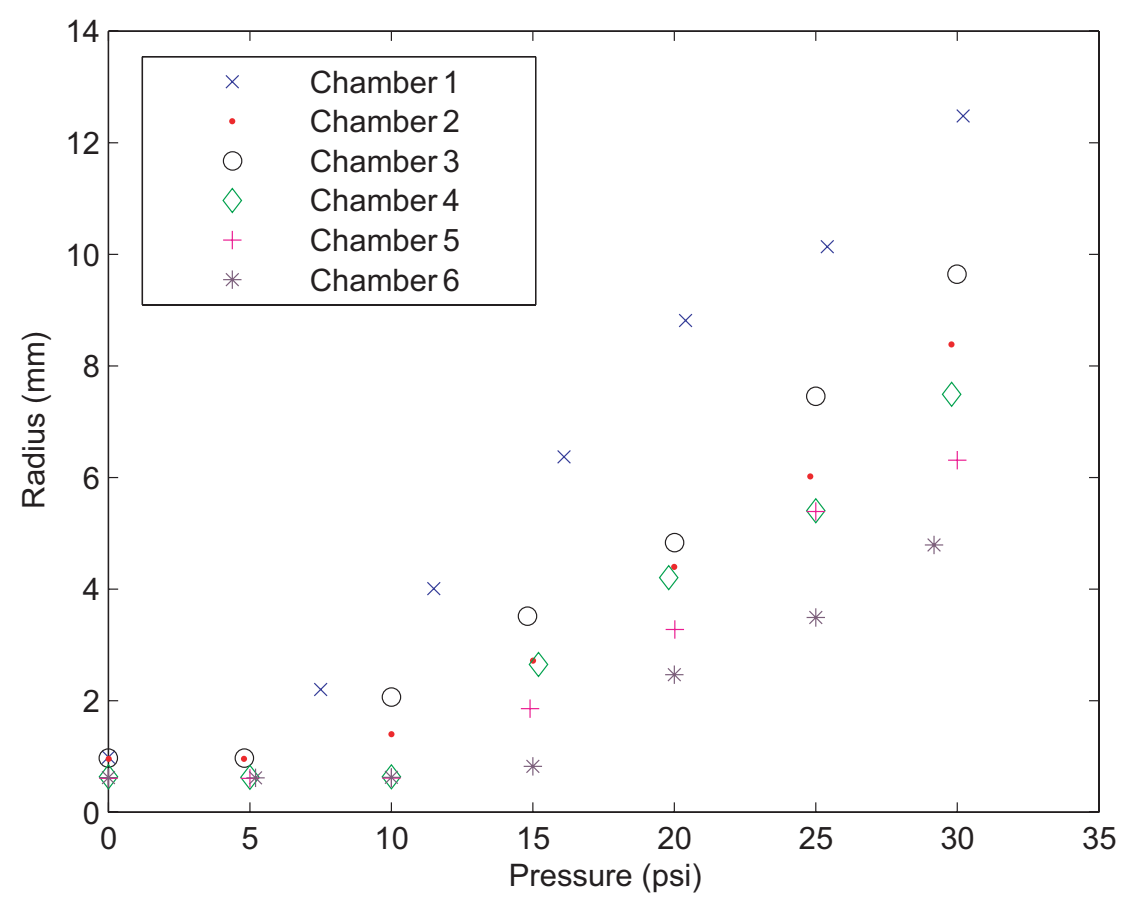

Figure 3.10 Radius increase vs pressure of several 1 $\mathrm{x}$ actuation chambers

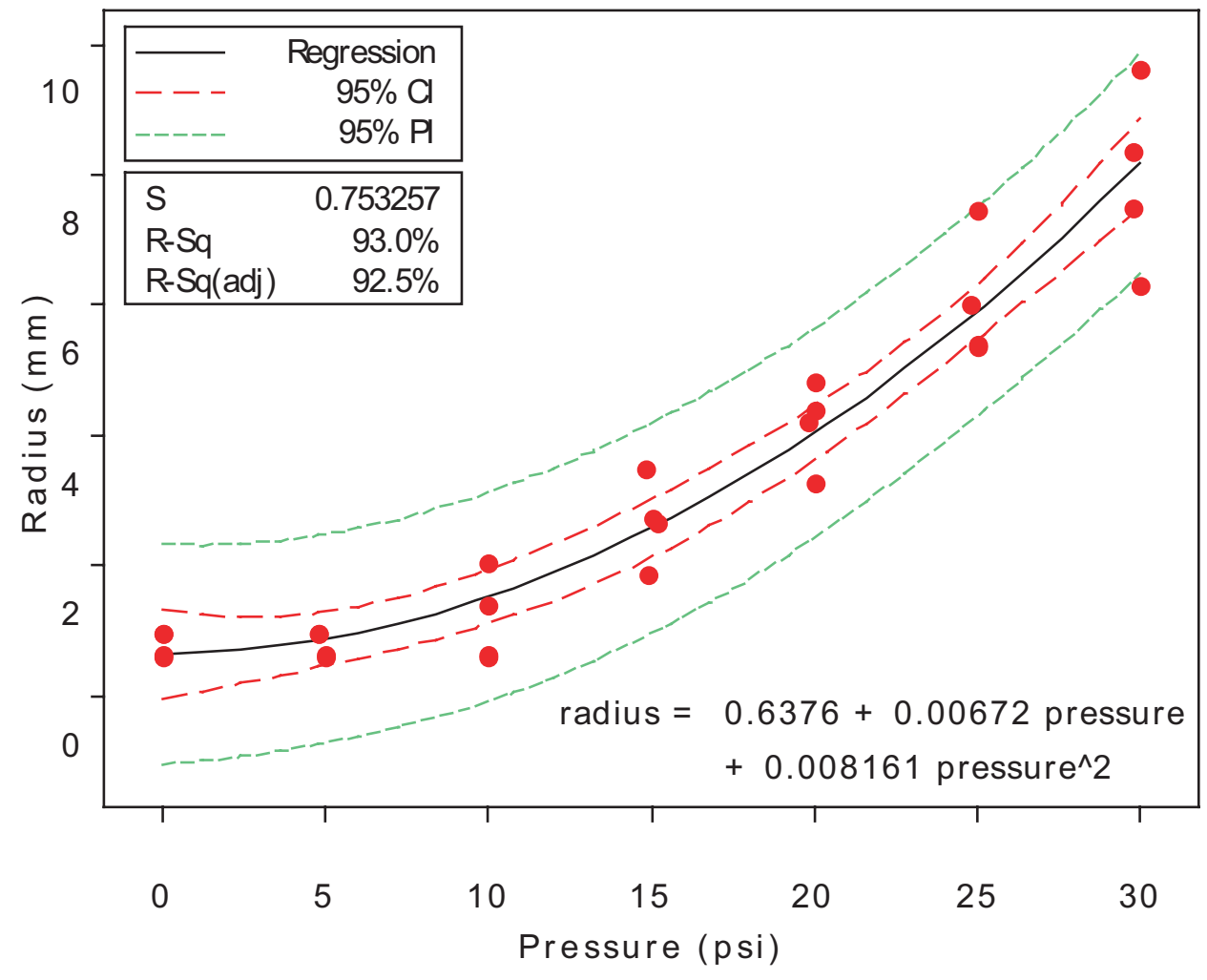

Figure 3.11 Chamber radius vs Pressure with a fitted 2nd order polynomial and computed confidence and probability intervals. 
reduced actuation and does not appreciably increase in radius before 20psi. These phenomena have been observed throughout the study with chambers of all sizes, but particularly in $1 \mathrm{x}$ chambers. These extremes in the actuation ability are likely related to the interaction of the inner walls of the chamber where some force or attribute of the chamber prevents the inner walls from separating to varying degrees. When this force is minimized, this represents an ideal condition for actuation as in the case of chamber 1. Poor actuation occurs when the force overcomes the strength of the lower internal fluidic pressures as in the case of chamber 6 .

In fact, a significant portion of chambers manufactured at the $1 \mathrm{x}$ size did not actuate at all, a condition termed "lock up". It is theorized that during manufacture the inner walls sometimes come into contact with each other. The degree of this contact results in both an increased surface attraction and a reduced space for fluid to move within the chamber. This could be the result of van der Waals forces or perhaps a build up of electrostatic charge within the polymer material. Alternatively, the chamber walls may be experiencing buckling behavior due to variability in the shape of the manufactured cross section. The net result from one or a combination of these potential factors is the inability for fluid to occupy space within the chamber which precludes the ability for actuation.

For increased actuation such as in the case of chamber 1, the walls maintain a separation from one another that provides an unobstructed fluidic pathway within the chamber. Qualitative evidence to this effect has been gathered where the fluidic pathway is kept open by filling the chambers with various oils or water during manufacturing to help in keeping the walls separated. While these devices demonstrate "enhanced" actuation ability, the fluids severely complicated manufacturing steps and interfered with bonds and adhesives making it not a viable option for improving performance. 
In the case of normal actuation, the data demonstrates a second order trend of the radius increasing with pressure as shown in Figure 3.11 where a fitted line has been added to chamber's 2 through 5 data. Plotted in Figure 3.12 are several $1 \mathrm{x}$ chambers manufactured at different radii. It is evident from the graph that the radius increase is dependent on the initial curvature of the chamber since the slope for each chamber increases with larger radius.

Another important chamber characteristic for the chambers is the ability to return to their manufactured shape post-actuation, or in other words, their springback ability. Loss of springback would impair the ability of the insertion tool to hug the inner wall of the cochlea. Figure 3.13 shows the percent increase in radius from the original manufactured radius after actuation to increasing pressures. Loss of springback occurs as plastic deformation takes place in the material as a result of the pressurization. Also shown in Figure 3.13 is the radius achieved relative to original radius when a vacuum is applied within the chamber. The application of vacuum inside the chamber brings the radius back to $6.5 \mathrm{~mm}$ on average. By the use of vacuum, negative actuation is possible. However, the implant needs to maintain its springback curvature after insertion into the cochlea in order to bring the electrodes within close proximity to the modiolus. Use of vacuum may be an effective technique during acute testing, but would not be practical for chronic tests or permanent implantation. Therefore, it may be necessary to design the chambers with a predictable loss of springback to maintain a modiolus hugging form.

\section{Cross Section Deformation with Pressurization}

Additional work done by Butala in his Master's thesis looked into the change in cross section shape of an actuation chamber upon pressurization [30]. Figure 3.14 illustrates the cross section shape from white light interferometric 


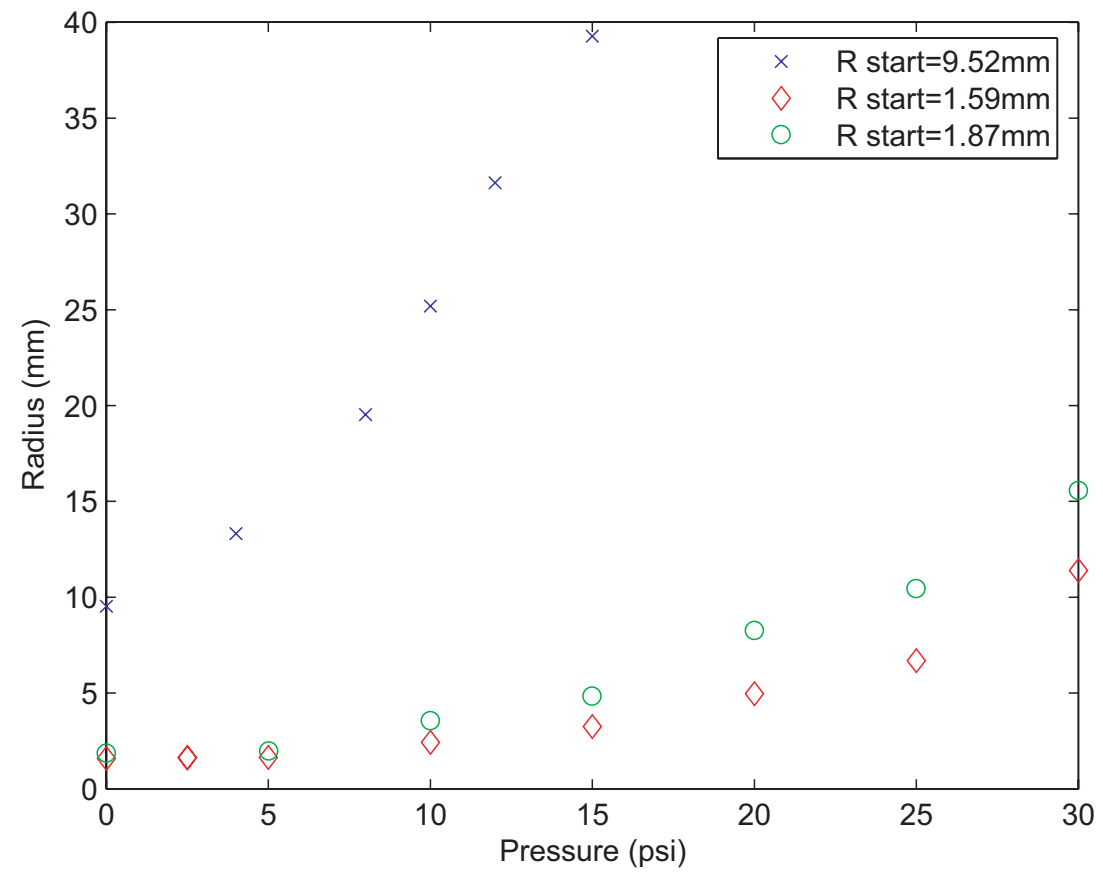

Figure 3.12 Chamber actuations for several starting radii.

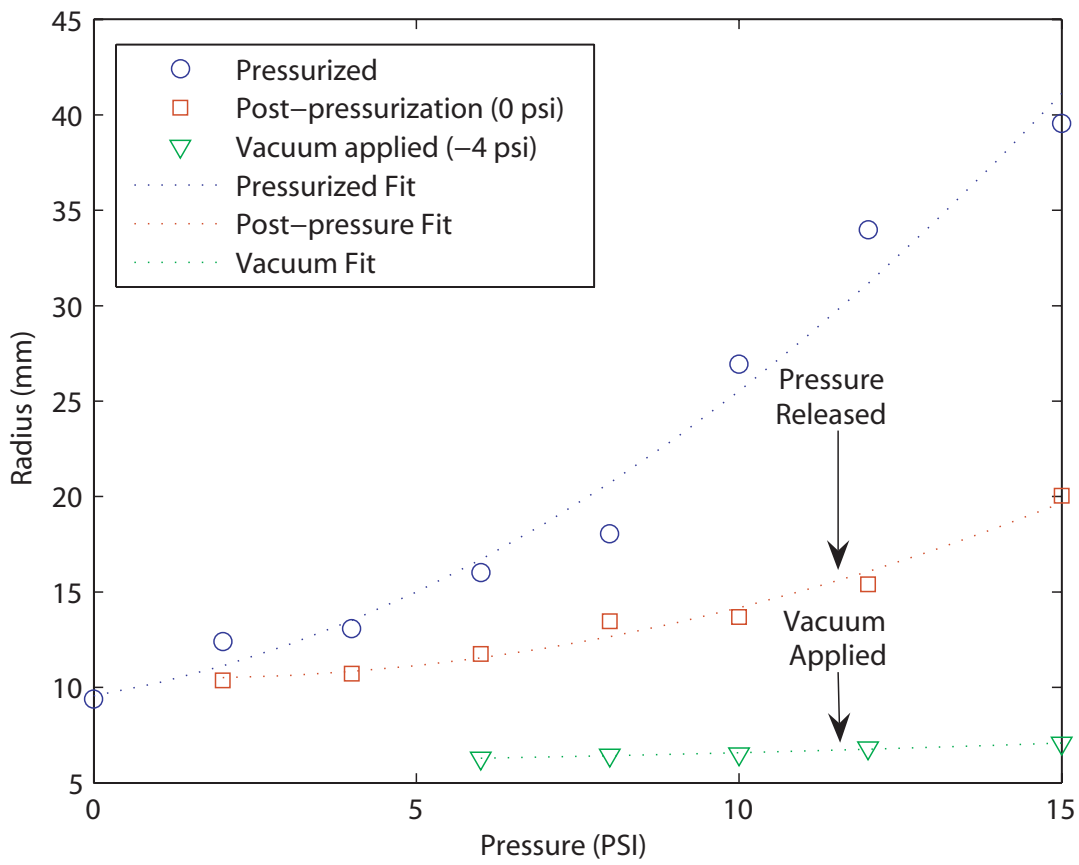

Figure 3.13 Loss of springback and radius achieved with vacuum. 


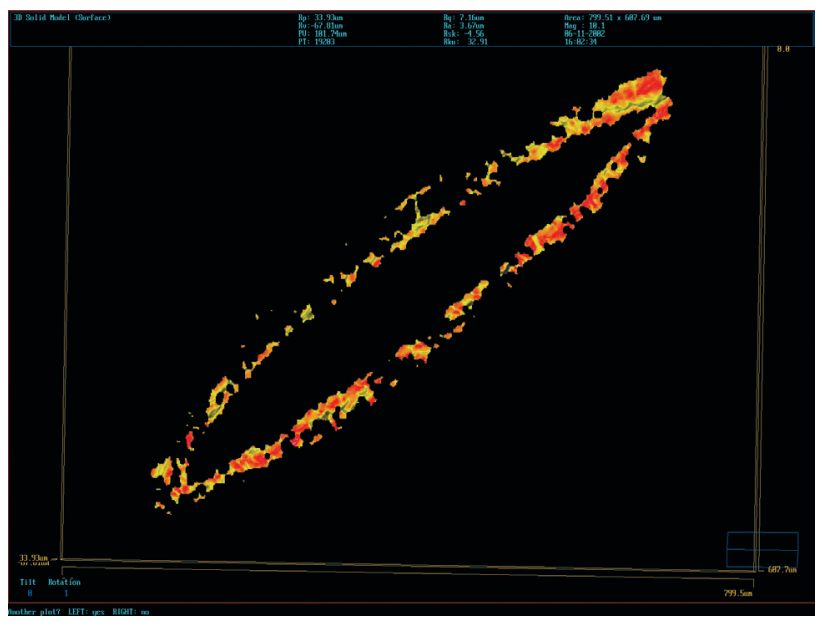

Figure 3.14 Interferometric microscope measurement of a PET chamber cross section $[30]$.
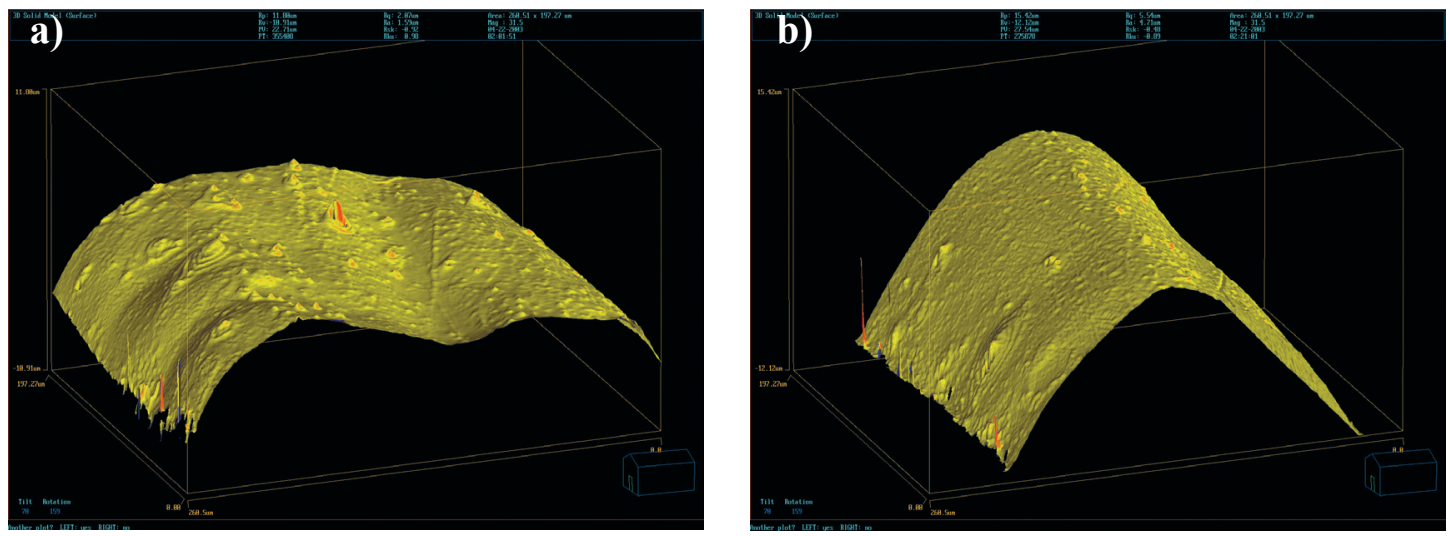

Figure 3.15 Cross section profiles at a) $0 \mathrm{kPa}$ and b) $70 \mathrm{kPa}$ (10psi) [30].

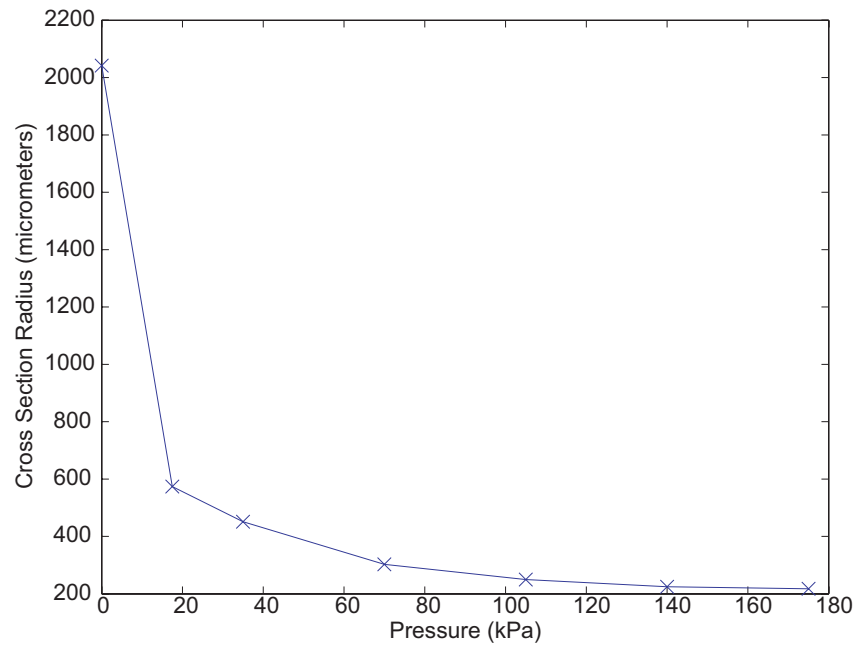

Figure 3.16 Chamber cross section radius during pressurization [30]. 
microscope readings of a flattened PET chamber at rest (0psi). The chamber was flattened from $560 \mu \mathrm{m}(0.022$ ") ID PET tube. As can be seen from the figure, the shape of the chamber after flattening is an ellipsoid with sharply crimped ends. The dimensions of the major and minor diameters are $800 \mu \mathrm{m}$ and $170 \mu \mathrm{m}$, respectively.

Butala also fabricated several chambers from $200 \mu \mathrm{m}(0.008$ ") ID PET tubing and coated them with a very thin layer of sputtered chrome to enhance the surface reflectivity. These were then mounted under the white light interferometric microscope in such a way as to observe the surface of the chamber as it is pressurized. Two of the graphs from this experiment are displayed in Figure 3.15ab (it should be noted that the axes in the figure are not to the same scale).

Readings were also taken at other pressures resulting in a graph of the cross section radius versus the applied pressure (Fig. 3.16). The cross section readings also show the tendency to lose the ability to spring back to its original shape since the final reading at zero pressure only returns to a $708.18 \mu \mathrm{m}$ radius while the original radius before pressurization was $2041.72 \mu \mathrm{m}$. The cross section measurements were performed using the MATLAB algorithm provided previously described for measuring overall chamber radius (Appendix C).

\subsubsection{FEA Modeling of the Fluidic Chambers}

In order to study the Bourdon type actuation phenomenon of the chambers, several finite element models (FEM) were created using the finite element software ABAQUS 6.4. The model geometry consists of a semicircular shell section of constant radius. The shell model is generated by sweeping a cross section shape along an arc. The cross section shape was defined by the data gathered from the interferometer measurements of a 1x PET tube resulting in the general dimensions of $240 \mu \mathrm{m}$ by $30 \mu \mathrm{m}$ (Fig. 3.14). 
Dimensions are not prescribed as a particular unit system in the ABAQUS software, but rather must remain consistent throughout the definitions of physical properties. Therefore, spatial dimensions were assumed in micrometers and other properties were converted to fit this convention (with all other units being customary SI units). For example, the units for pressure are $\mathrm{kg} / \mu \mathrm{m}^{*} \mathrm{~s}^{2}$, which corresponds to $10^{-6} \mathrm{~Pa}$.

Material properties were given as linear elastic and homogenous. The elastic modulus used was $4 \mathrm{GPa}$ with a Poisson's ratio of 0.4 and a yield strength of 250MPa. Material properties for PET were found from stress-strain test performed on the tubing [30]. Thickness of the material in the shell structure was initially defined as $3.86 \mu \mathrm{m}$ (the nominal thickness provided by the PET tubing manufacturer, Advanced Polymers) for early models, but later micrometer measurements of tubing in the manufactured state found that a thickness of $6.5 \mu \mathrm{m}$ is more appropriate.

Boundary conditions (BC) were prescribed in such a way as to simulate an isolated section of tubing. The fixed end had a symmetric BC to allow the cross section to change shape from the pressurization. A symmetric BC was also applied to the edges of the open side of the cross section. The computation time to solve the model is halved by modeling a half cross section and using the symmetric BC.

The $\mathrm{BC}$ for the free end of the tube section was problematic since a regular $\mathrm{BC}$ is fixed to the global coordinate system and cannot rotate as the large deflection of the tip occurs. The solution was to apply a kinematic coupling condition to the edges of the tip in which some motions of the edges are constrained to follow the motions of a reference node. The reference node was defined as the point where the upper and lower portions of the cross section come 
together since this point lies on the neutral axis and its motions would result in the straightening of the chamber rather than the deformation of the cross section.

The kinematic coupling constrained translation normal to the plane of the cross section and rotations into the plane of the cross section effectively creating a symmetric boundary condition that can move with the tip deflection. The kinematic coupling constraint was added through manipulation of the text input file that is submitted to the solver as the ABAQUS/CAE module cannot prescribe the condition.

Finally, a pressure load of $3 * 10^{-7} \mathrm{~kg} /\left(\mu \mathrm{m}^{*} \mathrm{~s}^{2}\right)(300 \mathrm{kPa})$ was applied to the entire inner surface. Figure 3.17 illustrates the geometry of the model and summarizes the BCs.

The model was meshed using quad shaped elements and the edges were seeded with an approximate spacing of $20 \mu \mathrm{m}$. The geometric order of the elements was set to quadratic to give each node 6 degrees of freedom.

A single step was defined with an initial increment step of 0.00135 and a minimum increment step of $1 * 10^{-5}$ (with total increments step times summing to 1 to complete the solution) and a maximum number of increments within the step of 10,000. Also, because of the large deformations, the nonlinear geometry parameter was set to require rotation of the pressure vectors to remain normal to the model surfaces during deformation.

Solutions of the finite element analysis (FEA) models show large deformation and degree of actuation. In Figure 3.18, the solution for a $10^{\circ}$ section of a semicircular chamber with a $1000 \mu \mathrm{m}$ radius and an arc is shown. The solution converged in approximately 60 seconds. 


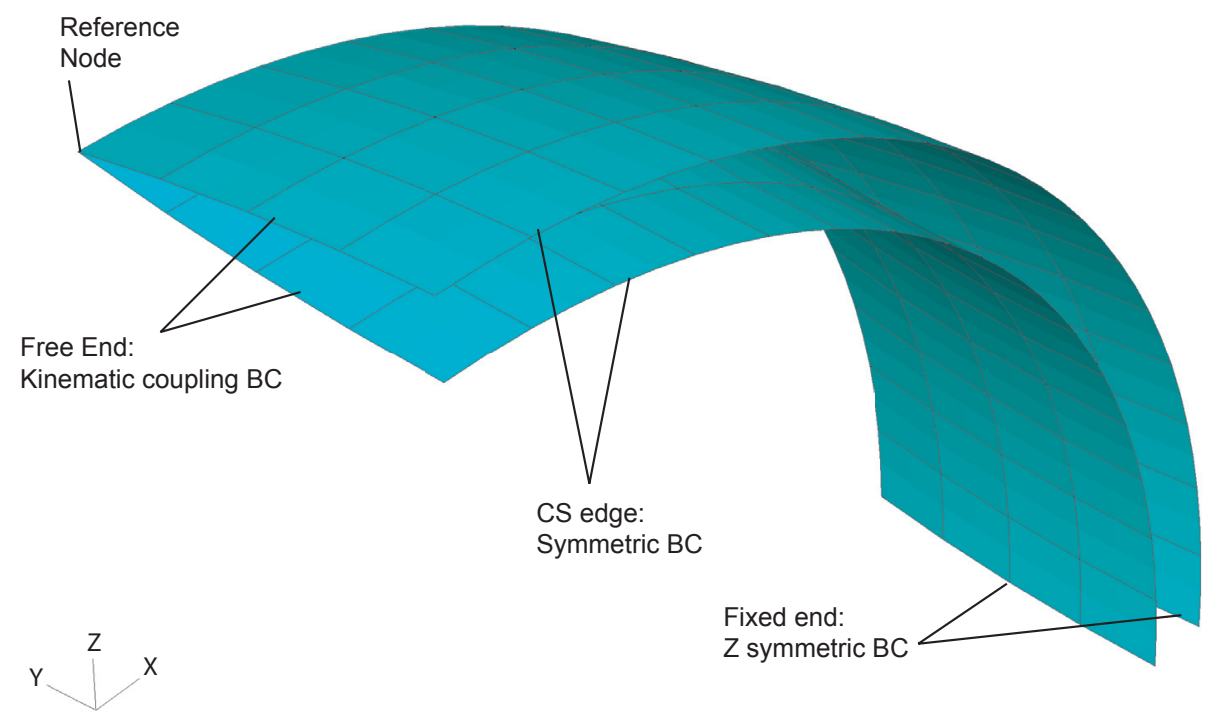

Figure 3.17 FEA model setup for chamber actuation $(R=1000 \mu \mathrm{m})$.

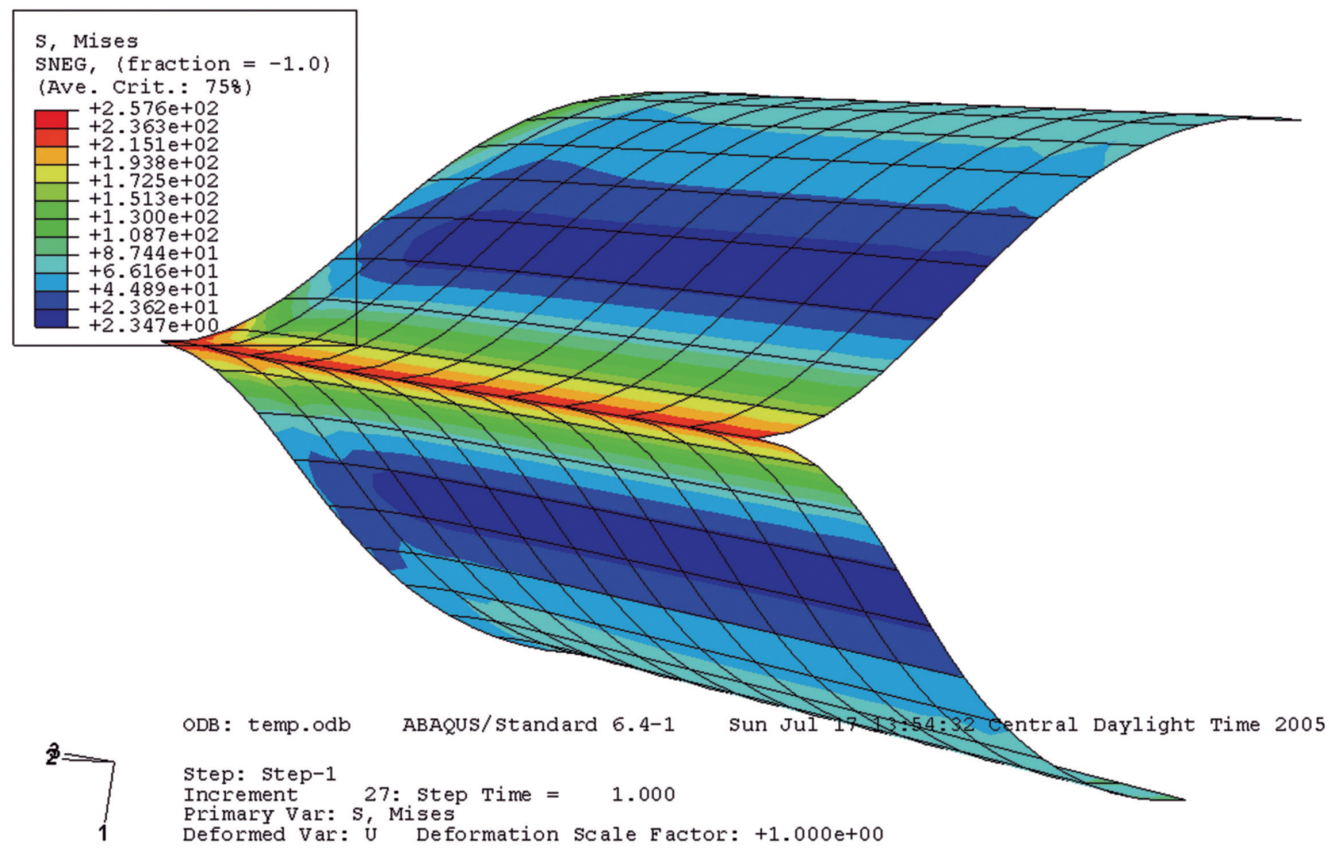

Figure 3.18 FEA results for a $10^{\circ}$ semicircular section with a $1000 \mu \mathrm{m}$ radius. 
The parallel stress bands in the model indicate that the $\mathrm{BCs}$ were applied successfully, essentially creating an isolated chamber section. Large stresses (maximum stress was $258 \mathrm{MPa}$, the yield strength) occur primarily at the crimped edge of the model. This indicates the location where plastic deformation occurs in the chamber resulting in reduced springback potential. The calculated plastic strain in the model is illustrated in Figure 3.19.

The deformed overall radius of the chamber can be estimated from the node coordinates at the fixed and kinematic boundaries. If a line is drawn from the outer radius symmetric edge node on the fixed end to the inner symmetric edge node on the fixed end, and the analogous operation is done on the kinematic end, the two lines should intersect at the center of curvature. Measuring from the center of curvature to the midline of the cross section yielded the results shown in Figure 3.20 for several starting radii of curvature.

Several observations of note can be made from Figure 3.20. First, in comparing each of the three FEA results, it is apparent that the slope of each line increases as the starting radius increases even though the cross section shape and size is identical in each case. This indicates that there might be an interaction between the cross section shape and the overall radius of curvature. Therefore, for modeling purposes, the differential cross section shape cannot be considered independently from the overall radius of the chamber.

Next, when comparing the FEA results for a chamber of $\mathrm{R}=1000 \mu \mathrm{m}$ with the fit line from experimental results it is apparent that the experimental results show more actuation at lower pressures, but begins to converge with the fitted results at higher pressures. Even more noteworthy is that the experimental results for the chamber with 


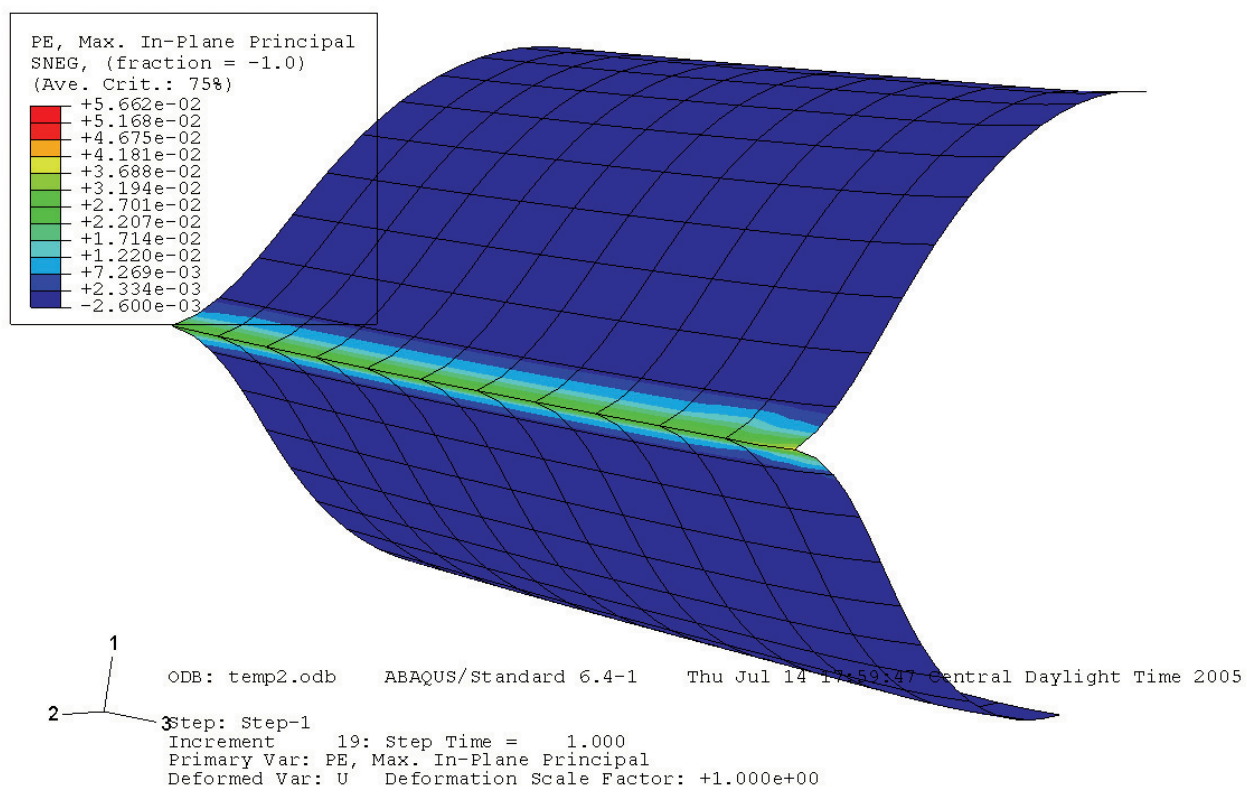

Figure 3.19 Plastic strain in the edge location for the FEM.

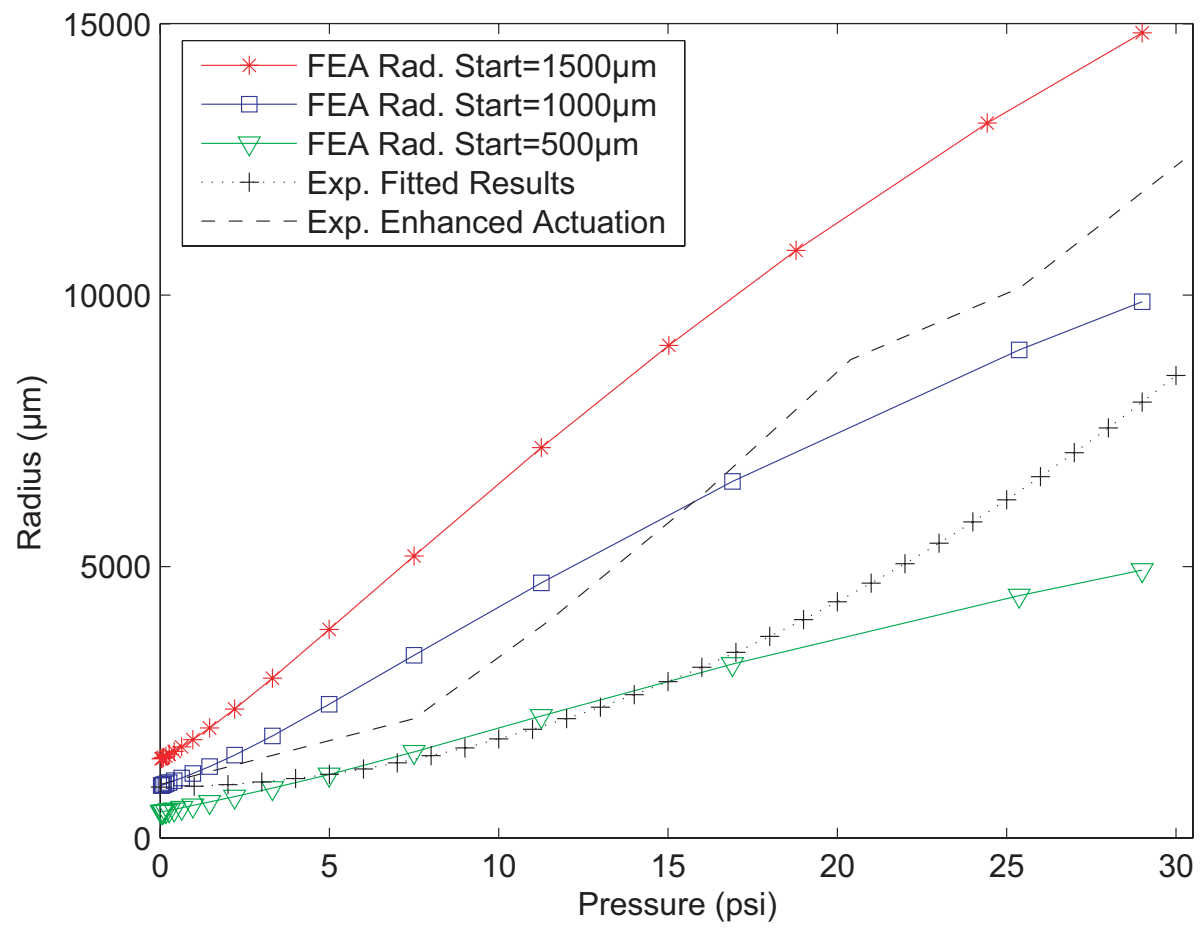

Figure 3.20 Actuated radius of curvature for several FEA chamber sections of varying starting radius versus experimental results. 
"enhanced" actuating ability is followed by the FEA results for the same starting radius.

This hints at the possibility that the FEA model is accurately portraying actuation of a chamber operating in the ideal or "enhanced" operating regime. Furthermore, if this is the case, then the "normal" operating regime represents an intermediate condition of "lock up", where internal chamber wall effects are still at work, but actuation is still possible.

Finally, it should be stated that the FEA model represents only a $10^{\circ}$ circular section of tubing while the experimental measurements were performed on circular sections of $180^{\circ}-270^{\circ}$. Additional physical effects likely come into play that will be discussed further in the next section on the theory of actuation mechanisms.

FEA models were attempted for larger circular sections and for helical sections, but they were found to be computationally cumbersome and often the complicated models failed to solve due to "local material instabilities" as reported by the ABAQUS software. A discussion with ABAQUS technical support suggested adding a stabilization factor to the model. The stabilization factor works as a damping factor to counteract the localized material instabilities. In practice, it was found that while the stabilization factor did correct the error, it also had an overwhelming affect on the deformations solved for in the model. Furthermore, since choosing the size of the stabilization factor is rather arbitrary (and therefore its effect on the deformations come into question), an alternative method was sought for solving full scale models that is both computationally less intensive and free from the burden of dubious correction factors.

\subsubsection{Theory for Mechanisms of Actuation}

As stated earlier, an adequate analytical model for the actuation for Bourdon 
tubes that could be used as a predictive tool for the large deflections needed in the implant tool was not found in literature. But, by coupling results from FEA models along with geometric and mechanics models, a possible method for the prediction of actuation results is proposed and tested against experimental results.

The method theorizes two primary components of the actuation. The first and foremost is the geometric change in the chamber cross section. This can be understood if we look at the material of the chamber at its most circumferential and central areas in the radial direction from the center of curvature. As the pressure increases, the cross section changes, pushing the center of the most circumferential wall out farther and pushing the most central wall towards the center of curvature. But, since the sidewalls keep the outer and inner walls parallel to one another, the radius must increase to compensate.

A mechanics model for the change in cross section would most likely be quite complex and even if found, most likely could only be made to fit geometrically simple cross sectional shapes. But, the cross section deformation is easily solvable using FEM as has been previously shown, and is easily adaptable to arbitrary cross section shapes.

Therefore, for the tapered, helical shape needed for the cochlear insertion tool, the actuation can be modeled if the helix is divided into discrete arcs of constant radius and the deformations for each arc-section can be solved and then combined to give the overall change in shape of the helical device.

The second component of actuation can be applied in addition to the first calculation from the geometric shape change. This component results from the area differential between the outer and inner chamber walls (again referring to the radial direction). This difference of area between the 
outer and inner walls with the internal pressure acting on it results in a net distributed load on the chamber directed radially outward. The helical chamber can then be modeled as a beam with a radially directed distributed load.

This is a mechanics problem that has been previously solved (Equations 3.1-3.6). Rourke and Young's solution for a distributed load on a cantilever curved beam can be applied to find tip displacement [35]. The formulation is as follows for an elliptical cross section:

$a$ - major axis half width of the cross section

$b$ - minor axis half width of the cross section

$t$ - wall thickness

$P$ - pressure

$\theta$ - angular extent (half angle)

$w$ - distributed load

$I$ - area moment of inertia

$A$ - cross-sectional area

$R$ - arc radius

$F$ - cross section shape factor

$G$ - shear modulus

$E$ - elastic modulus

$v$ - Poisson's ratio 
Radial component of internal pressure in the cross section:

$$
Q=2 P \int_{0}^{a} \frac{\sqrt{\left(1-\frac{x^{2}}{a^{2}}\right) a^{2} b^{2}}}{x b^{2} \sqrt{1+\frac{\left(1-\frac{x^{2}}{a^{2}}\right) a^{4}}{b^{2} x^{2}}}} d x
$$

Distributed load due to pressure differential:

$$
w=-\frac{((R+b) \theta-(R-b) \theta) Q}{R \theta}
$$

Axial stress deformation factor:

$$
\alpha=\frac{I}{A R^{2}}
$$

Transverse shear deformation:

$$
\beta=\frac{F E I}{G A R^{2}}
$$

Horizontal and vertical loading terms for a uniform radial load:

$$
\begin{gathered}
L F_{H}=w R\left[\begin{array}{l}
3 \sin \theta-\sin ^{3} \theta-3 \theta \cos \theta \\
+(\alpha-\beta)(\sin \theta-\theta \cos \theta) \\
+(\alpha-\beta) \sin ^{3} \theta
\end{array}\right] \\
L F_{V}=w R\left[\begin{array}{l}
3 \theta \sin \theta-3 \sin ^{2} \theta \cos \theta \\
+(\alpha+\beta) \theta \sin \theta \\
-(\alpha-\beta) \sin ^{2} \theta \cos \theta
\end{array}\right]
\end{gathered}
$$

Tip displacement for a cantilever arch:

$$
\delta_{H A}=\frac{-R^{3}}{E I} L F_{H}
$$




$$
\delta_{V A}=\frac{-R^{3}}{E I} L F
$$

Solving the problem using this formulation requires dividing the chamber curvature into small arc sections, calculating the deflection, then summing the results to determine the final shape. Alternatively, the problem could also be solved by defining an FEA model using beam elements instead of using the Rourke and Young solution. In this case, the beam stiffness and distributed load could then be directly solved for from the FEM output on the cross section deformations. This approach could constitute a method for quickly computing the performance for actuating devices with alternative configurations and materials.

\subsection{Computational Model for Insertion Tool Actuation}

The overall aim for a computational model is to reasonably approximate the actuation characteristics for chambers with user-defined parameters. The model is written as multiple functions in MATLAB Release 13 and can be called manually from the main control window or from another MATLAB program or function (see Appendix D). Portions of the model are computed and solved as ABAQUS 6.4 finite element models. In these instances, MATLAB creates a text script document formatted as an ABAQUS input file and then calls the ABAQUS command module to solve the FEM defined in that file.

The computational modeling process begins by defining the pertinent parameters used to create the model. These parameters include:

- Nodal coordinates for the chamber centerline ( $x, y, z$ coordinates), $\left(N_{C L}\right)$

- Nodal coordinates for the chamber cross section shape ( $a, b$ coordinates), $\left(N_{C S}\right)$, with the origin $(0,0)$ defining the centerline relationship to the cross 
section

- Chamber material properties: thickness $(t)$, elastic modulus $(E)$, Poisson's ratio $(v)$

- Internal pressure, $(P)$

The geometry of the model is basically a $3 \mathrm{D}$ centerline curve that defines the coordinates of the chamber's centerline axis. Each section between defined points can be thought of as having a cross section shape assigned to it, where the a-direction of the cross section corresponds to the z-direction of the centerline coordinate system.

The modular design of the model written as multiple functions allows flexibility when solving a model. Each individual component function can be called separately as needed. In some cases, it may be beneficial to obtain and then modify results individually before then passing those results onto the next component. An overview of the modules and their generalized function is shown in Figure 3.21.

\subsubsection{Module 1: Cross Section Deformation}

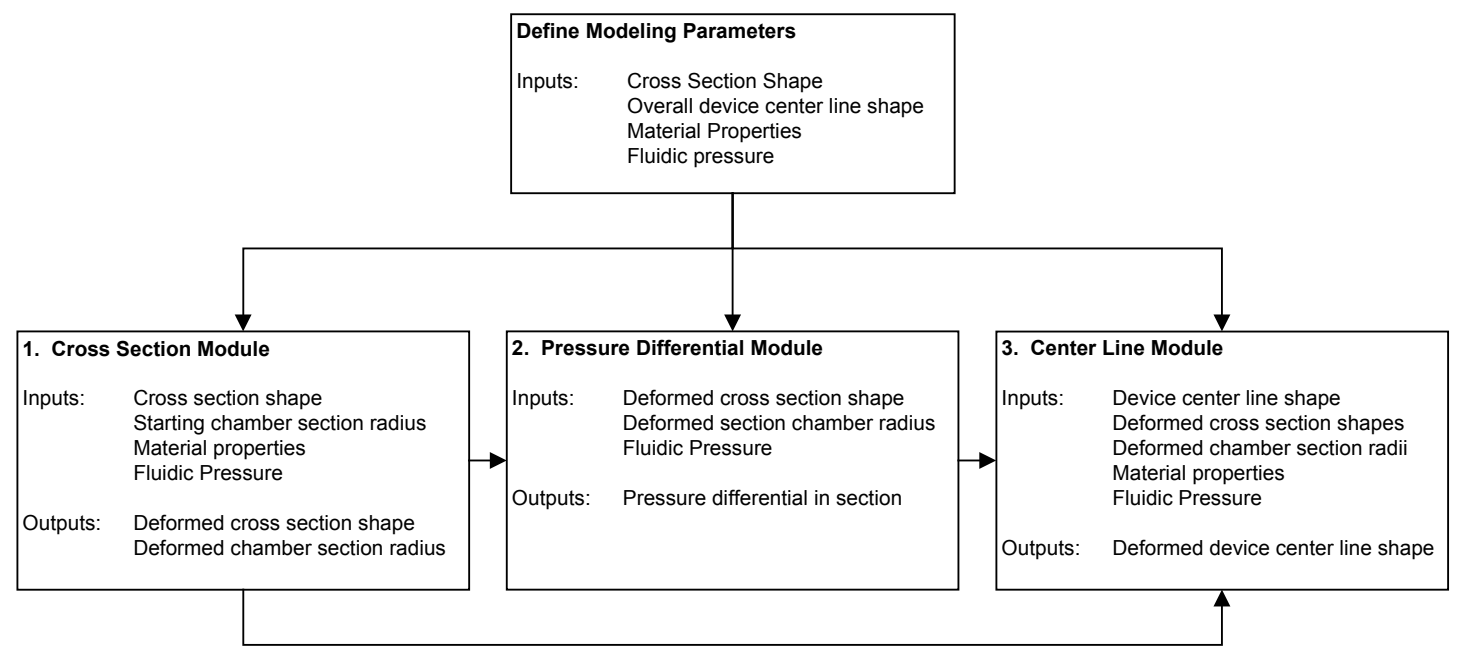

Figure 3.21 Overview of modules in the computational model. 
The first module takes the user parameters for the cross section and then calculates the 3-dimensional nodal coordinates of the cross section rotated through space in small increments over a total arc of 10 degrees with radius $\mathrm{R}$. The incremental arc length between nodes is defined by using the average length between user defined cross section nodes. This ensures that each node is approximately equal distance from each adjacent node if the user-defined cross section's nodes are also evenly spaced.

Intermediate nodes are then calculated and the connectivity between all nodes is determined (i.e., elements are defined). Next, lists of nodes and elements involved with certain boundary conditions are established (see Figure 3.17 for $\mathrm{BCs})$. The information is then scripted using the ABAQUS input file format and written to a text file (named by the user) with the extension ".inp".

ABAQUS is then called to solve the input file interactively, meaning that the MATLAB program flow halts while ABAQUS solves the FEM and outputs progress text to the MATLAB command window. A binary output file (*.fil) made up of 64-bit words is created containing the ABAQUS solution information and deformed nodal coordinates corresponding to each incremental step in the solution.

The ABAQUS output file is read into MATLAB which then performs calculations to estimate the deformed radii of the cross section at each step increment. The calculations involve the simple solution for the intersection of two lines defined by the chamber's ends as was previously described in Section 3.3.2.

\subsubsection{Module 2: Deformation from the Pressure Differential}

The distributed load factor $(d l f)$ for differential pressure between the inside radius 
and the outside radius is calculated by summing the elemental unit vector components in the radial direction $\left(u_{j}\right)$ multiplied by the projected length of each element onto a plane perpendicular to the radial direction in the deformed cross section $\left(E_{i}\right)$ multiplied by two with the entire summation divided by the radius, $R$. Written algebraically:

$$
d l f=\frac{\sum\left(2 u_{j} E_{j}\right)}{R}
$$

The distributed load factor is then multiplied by the pressure $(P)$ to resolve the outward distributed load on the cross section as a result of the pressure differential between the centrally and circumferentially located walls of the cross section.

\subsubsection{Module 3: Overall Chamber Centerline Deformation}

The distributed load, and radius change due to cross section deformation information along with the user defined nodes for the chamber centerline, can then be passed onto a function to build the ABAQUS input file for the overall deformation of the chamber along its centerline. The function first transforms the xyz-coordinates for the centerline nodes into a series of arcs. Each arc has its end points situated on a pair of consecutive nodes in the centerline with the constraints that each arc must be tangent to its adjacent arc $(s)$ and that the radial line for the arc is perpendicular to the $\mathrm{z}$ axis. The first constraint ensures that each arc forms a smooth curve with neighboring arcs and the second constraint keeps the curve helically constrained.

The increase in radius found from the cross section definition is then applied to the individual arcs (or several radius changes in the case where arc radii vary). The deformed

arcs are then transformed back to Cartesian coordinates for the deformed node positions.

The function then builds an ABAQUS formatted script file which defines the 


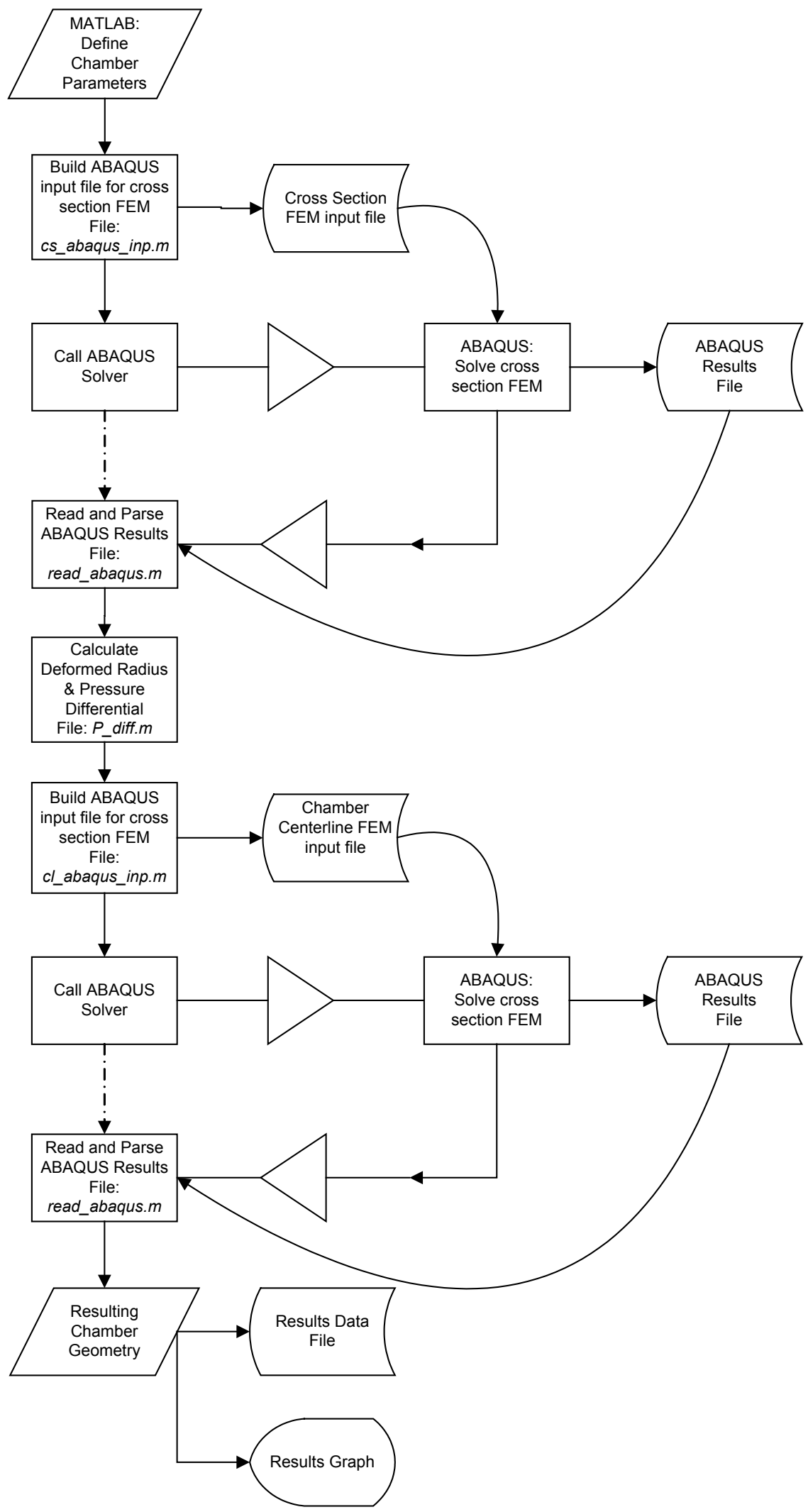

Figure 3.22 Program flow for chamber actuation computational model. 


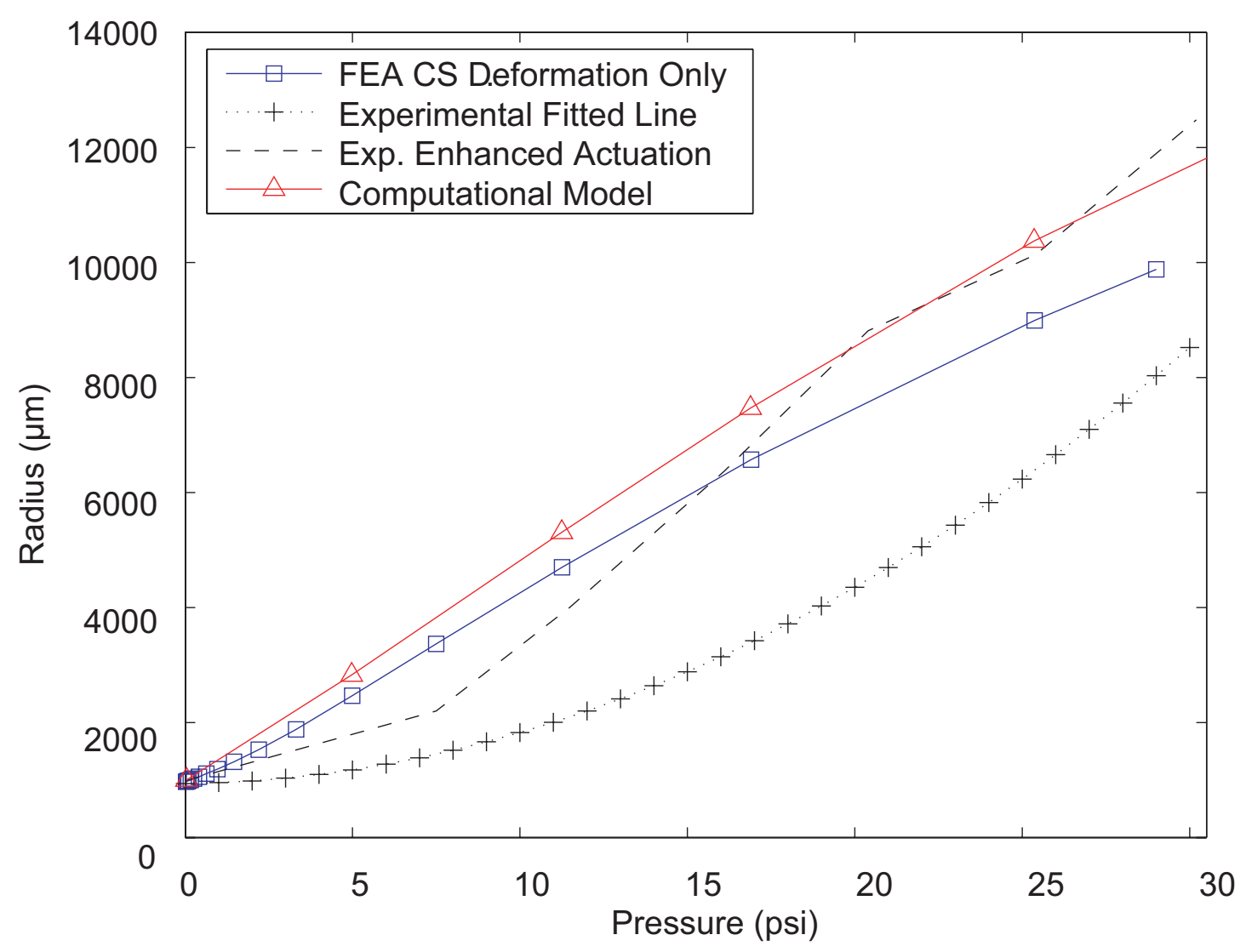

Figure 3.23 Results from the computational model vs. experimental results.

deformed centerline curve as a beam with a cross section (defined by the deformed cross section nodes previously solved) and the distributed load applied. Control is again passed to ABAQUS to solve the FEM and the resulting deformed coordinates are read back into MATLAB to complete the final resulting configuration for the chamber shape.

A detailed flow chart for a simple actuation model where there is only one cross section shape and the chamber centerline for the model is composed of only one radius is illustrated in Figure 3.22. 


\subsubsection{Comparison of the Model With Experimental Measurements}

Using the computational model for a chamber with an overall radius of $1000 \mu \mathrm{m}$ and a $180^{\circ}$ arc for a chamber centerline, the results show an improved fit with the enhanced data from the experimental results over the previous fit when simply modeling deformation due to cross section change (Figure 3.23). The increased radius for the computational model over the FEM cross section model is the result of the addition of the distributed load from the pressure differential component of actuation being added.

Besides more closely matching experimental results, the computational model has the added capability of modeling complex, 3D centerline geometries. However, for complex centerline geometries comprised of many nodes with varying radii, the solver would take up to several minutes per centerline element, possibly requiring hours to solve for the entire model with a complex centerline geometry.

One option to reduce the computational time required for solving a complex model is to pre-solve for a number of cross section radii and pressures to create a look-up table from which values could be interpolated. The method for applying this approach in a design study would be to pre-choose several of the chamber parameters (e.g., material, cross section shape) and then pre-solve over a range of likely input pressures. The results could then be used to supply input information to solve for the final shape of a complex 3D centerline by quickly interpolating to supply inputs to the second portion of the computational model (beginning with: Build ABAQUS input file).

A more efficient and practical method similar to the look-up table is to use the principles from design of experiments (DOE) and orthogonal factorial design spaces to reduce the number of runs needed to create a presolved design space. Methods and benefits to applying DOE to build design 
models to aid in computational modeling are discussed in the following section.

\subsubsection{Creating a Model Space Using DOE}

In DOE, a 2-level factoral orthogonal design takes advantage of overlapping parameter settings to gain as much additional information as possible about how the parameters behave between the levels. It also uses statistical analysis to gauge how significant those parameters are for defining the design space as well as the interactions between multiple parameters. If we wish to vary 3 factors for instance, the DOE requires the computational model to make runs for upper and lower boundary levels for each of those settings, or 8 runs $\left(2^{3}\right)$. Alternatively, if basic linear interpolation were employed, we would like several intermediate measurements at the minimum between each of the levels for sufficient spacing of response data in order to interpolate accurately. Adding 3 intermediate levels for a 3 factor results in at least 125 runs $\left(5^{3}\right)$, or 16 times more data points required in a similar DOE model.

An initial DOE model was created, and five parameters were chosen to learn of their effects on the computational model. Of specific interest were the responses of computed deformed radius and $\mathrm{CPU}$ time required to solve models of simple $180^{\circ}$ constant radius semicircular chambers when those five factors are varied. The chosen parameters are outlined in Table 3.1:

Table 3.1 DOE Parameters and Levels

\begin{tabular}{|c|c|c|c|}
\hline Parameter & Units & Lower Level & Upper Level \\
\hline Start Radius & $\mu \mathrm{m}$ & 500 & 4000 \\
\hline Wall Thickness & $\mu \mathrm{m}$ & 1 & 7 \\
\hline Pressure & $\mathrm{kg} / \mu \mathrm{m}^{*} \mathrm{~s}^{2}$ & 0.03 & 0.3 \\
\hline CS Ratio & & 5 & 40 \\
\hline Nodes & & 5 & 10 \\
\hline
\end{tabular}


Table 3.2 DOE Run Settings and Response Results

\begin{tabular}{|c|c|c|c|c|c|c|c|}
\hline Run & $\begin{array}{c}\text { Start } \\
\text { Radius }\end{array}$ & $\begin{array}{c}\text { Wall } \\
\text { Thickness }\end{array}$ & Pressure & CS Ratio & Nodes & $\begin{array}{c}\text { Final } \\
\text { Radius }\end{array}$ & $\begin{array}{l}\text { CPU } \\
\text { Time }\end{array}$ \\
\hline & $\mu \mathrm{m}$ & $\mu \mathrm{m}$ & $\mathrm{kg} / \mu \mathrm{m} * \mathrm{~s}^{2}$ & & & $\mu \mathrm{m}$ & $\mathrm{sec}$ \\
\hline 1 & 4000 & 1 & 0.3 & 40 & 5 & 965.52 & 35.047 \\
\hline 2 & 4000 & 1 & 0.3 & 5 & 10 & 8339.1 & 63.828 \\
\hline 3 & 4000 & 1 & 0.03 & 5 & 5 & 862.37 & 19.813 \\
\hline 4 & 500 & 7 & 0.03 & 40 & 10 & 8255 & 115.421 \\
\hline 5 & 500 & 7 & 0.03 & 40 & 5 & 12152 & 285.016 \\
\hline 6 & 4000 & 7 & 0.3 & 5 & 5 & 5460.2 & 11.047 \\
\hline 7 & 500 & 1 & 0.3 & 40 & 10 & 20111 & 66.093 \\
\hline 8 & 500 & 7 & 0.3 & 40 & 5 & 1378.9 & 29.578 \\
\hline 9 & 500 & 1 & 0.03 & 40 & 5 & 11891 & 38.266 \\
\hline 10 & 500 & 1 & 0.03 & 5 & 5 & 862.96 & 11.00 \\
\hline 11 & 500 & 1 & 0.03 & 5 & 10 & 12205 & 226.016 \\
\hline 12 & 500 & 1 & 0.03 & 40 & 10 & 102080 & 281.734 \\
\hline 13 & 4000 & 7 & 0.03 & 40 & 5 & 7742.8 & 125.875 \\
\hline 14 & 4000 & 1 & 0.03 & 40 & 5 & 44242 & 49.25 \\
\hline 15 & 4000 & 7 & 0.03 & 5 & 5 & 7788.4 & 35.593 \\
\hline 16 & 500 & 7 & 0.3 & 5 & 10 & 94925 & 225.531 \\
\hline 17 & 500 & 7 & 0.03 & 5 & 10 & 94534 & 785.204 \\
\hline 18 & 4000 & 7 & 0.3 & 5 & 10 & 1382.3 & 51.781 \\
\hline 19 & 4000 & 7 & 0.3 & 40 & 10 & 942.83 & 19.25 \\
\hline 20 & 500 & 7 & 0.3 & 5 & 5 & 5463.5 & 23.64 \\
\hline 21 & 4000 & 1 & 0.03 & 5 & 10 & 480.99 & 10.25 \\
\hline 22 & 4000 & 7 & 0.03 & 40 & 10 & 13362 & 156.969 \\
\hline 23 & 500 & 7 & 0.3 & 40 & 10 & 4387.7 & 29.438 \\
\hline 24 & 500 & 7 & 0.03 & 5 & 5 & 481.48 & 26.625 \\
\hline 25 & 2250 & 4 & 0.165 & 22.5 & 7.5 & 1427 & 53.188 \\
\hline 26 & 4000 & 7 & 0.3 & 40 & 5 & 4384.8 & 101.14 \\
\hline 27 & 500 & 1 & 0.3 & 5 & 5 & 13297 & 449.89 \\
\hline 28 & 4000 & 7 & 0.03 & 5 & 10 & 11958 & 91.812 \\
\hline 29 & 4000 & 1 & 0.3 & 5 & 5 & 939.54 & 11.672 \\
\hline 30 & 500 & 1 & 0.3 & 40 & 5 & 100270 & 859.829 \\
\hline 31 & 500 & 1 & 0.3 & 5 & 10 & 12524 & 46.954 \\
\hline 32 & 4000 & 1 & 0.3 & 40 & 10 & 12447 & 94.375 \\
\hline 33 & 4000 & 1 & 0.03 & 40 & 10 & 43751 & 173.047 \\
\hline
\end{tabular}



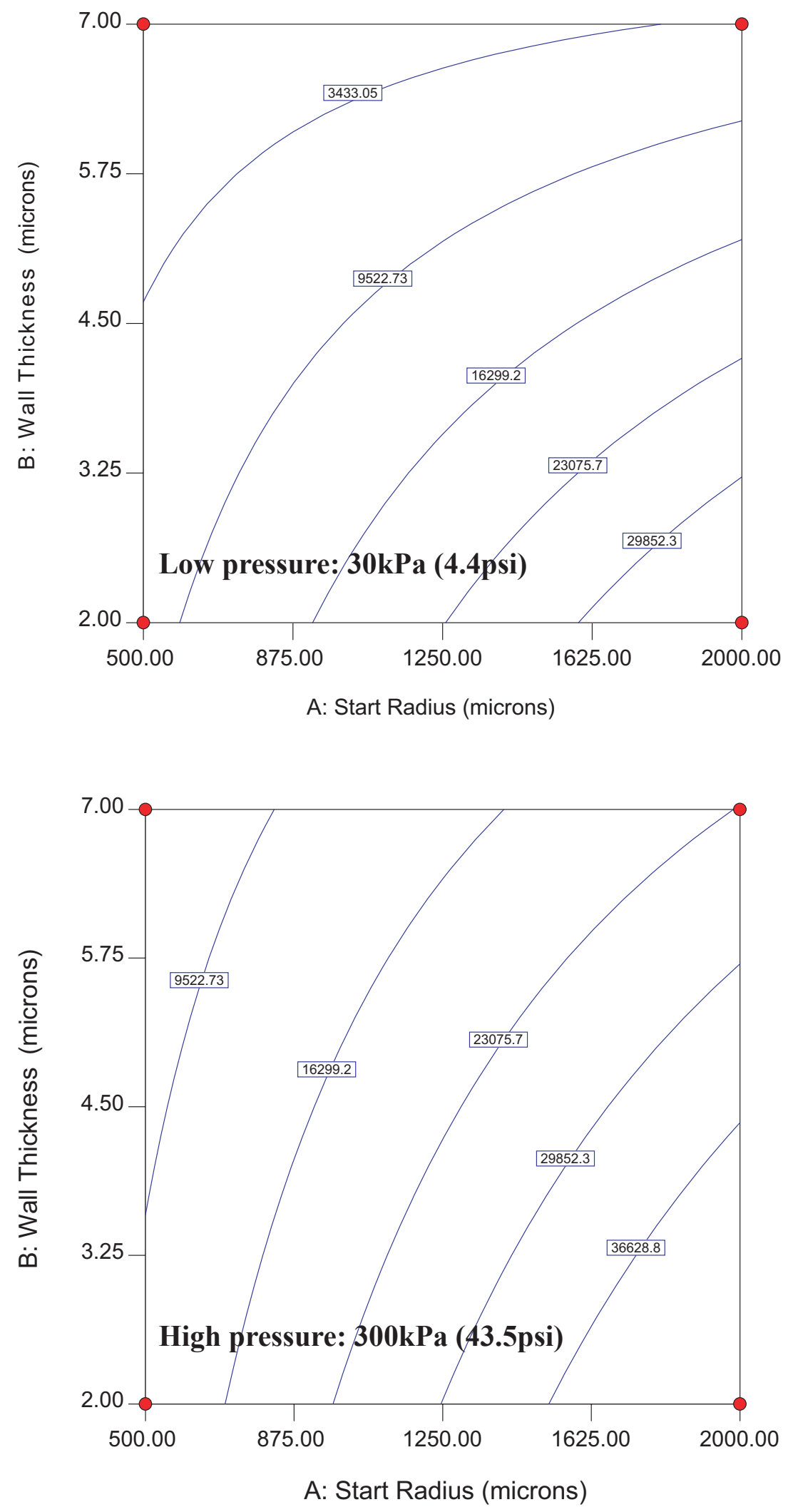

Figure 3.24 DOE model of final chamber radius by input parameters starting radius, wall thickness, and low and high pressures. 
The DOE model was created using the software Design Expert 7. For a basic 2-level, five parameter factorial design, 32 runs are needed with one additional run to check for model curvature in the center of the design space. The computational model was setup and run using the parameter levels specified by the DOE software. The results are listed in Table 3.2.

The DOE software found that the number of cross section nodes was insignificant to the model while all the other factors were significant. Furthermore, second order interactions between factors were also found to be significant.

When designing actuation chambers, cross section is most likely going to be specified and remain as a constant through most of the chamber. Also, since the number of cross section nodes specified in the FEM is insignificant to the final radius, the DOE model can therefore be further simplified to focus on the remaining three parameters of most interest. Figure 3.24 illustrates how final chamber radius behaves, which is algebraically described though the following equation:

$$
R_{\text {final }}=\left(\begin{array}{l}
27.03070 R_{\text {start }}+167.20703 t+4742.86755 P \\
-3.59833 R_{\text {start }} t+10.47097 R_{\text {start }} P+129.57856 P t \\
+3.19434 R_{\text {start }} P t-2986.24819
\end{array}\right)
$$

Five random parameter sets within the design space were chosen and run in the computational model to check the validity of the DOE modeling equation. The average percent error between the DOE model and the computational model was 3.1\% with the maximum error being $4.1 \%$. It is therefore practical and beneficial to use DOE methods to quickly develop a design model to solve for chamber cross section deformations.

\subsubsection{Summary of Computational Methodology}

Although the complexity of the computational method may seem daunting, it can 
be broken down to a few, fundamental ideas. The MATLAB and ABAQUS software are used to solve the actuation in two steps. The first step solves for a specific cross section with a specific and constant overall radius. The second combines one or several of the solutions from step one along with a distributed load from the pressure differential between the inner and outer walls and finds the final deformation of the chamber.

Because a chamber may have many different centerline radii along its length and we may want to solve for the actuation at a number of pressures, it becomes beneficial to create a solved design space of values for the cross section deformation to save computing time. This can be done either as a look-up table that can be interpolated, or more efficiently with a DOE model space. The design space can then be used to quickly pass values to modules 2 and 3 (Fig. 3.21) to solve a complicated chamber shape.

\subsection{Method for Applying Computational Model to an Insertion System}

Combining articulated control to a cochlea electrode array through the use of actuation chambers provides previously unattainable shape control and abilities for the surgeon performing the insertion procedure. Given these tools, a new method for performing the operation and positioning the device must be envisioned in order to employ them to their maximum advantage. Adding mechanical shape control to the electrode array no doubt allows for improved form-fitting to the spaces within the cochlea, but how can a surgeon constantly adjust the chamber pressures to match the modiolus without the benefit or knowledge of an inserted device's position or shape?

The proposed solution is to create a computer controlled device whereupon the computer dictates the chamber pressures to adjust the implant shape. The system configuration would use a feedback control loop to constantly adjust

pressure as the surgeon moves forward with the insertion. Estimates of the 
device's shape can be calculated using the readings from the strain gages along the probe's length. Tip and edge sensors on the probe can detect contact forces. The computer would then use this information to calculate adjustments to the shape of the device and provide graphical position and shape information to the surgeon. 


\subsection{Fabrication Methods and Results}

The methods used to fabricate the actuation tool were developed over many iterations of testing and experimentation. The challenges of fabricating a device with sub millimeter-sized features and a complex, helical shape were overcome as each problem presented itself. The following sections detail the methods used to fabricate fluidically actuated tools for the cochlear implant.

\subsection{Development of the Fabrication Process}

The first challenge was to choose a material that could be manipulated to produce the desired features while meeting requirements for biocompatibility, strength and flexibility. Commercially available PET (polyethylene teraphthalate) heat-shrink tubing met these requirements. Furthermore, the tubing is clear allowing for use of medical grade UV cure adhesives for bonding components.

PET tubing has been used in applications for encapsulating and binding components as well for manufacturing portions of catheter balloons used in the medical industry. The PET tubing was obtained from Advanced Polymers Inc. and is available in hundreds of stock sizes ranging down to tubing with a $200 \mu \mathrm{m}(0.008 ")$ inner diameter(ID) and a $0.00015 "(3.8 \mu \mathrm{m})$ thick wall.

The heat-shrink ability provides a method for altering the shape of the material. Shrinking occurs as low as $70^{\circ} \mathrm{C}$ where the tubing will shrink approximately $5 \%$ in both the longitudinal and circumferential directions. Increasing the temperature to $190^{\circ} \mathrm{C}$ will further shrink the material up to a total of $20 \%$. Reduction of the tubing diameter in the radial direction can be enhanced by drawing the tubing along its axis which will allow for shrinking up to $70 \%$ when combined with heat [31]. 
By constraining the tubing to a desired configuration and heating, the tubing will then be deformed to that shape. This is the basic fabrication principle for making the actuation chambers of the backing device. The underlying mechanics principle behind this is that when the tubing is forced into a certain shape, areas of tensile and compressive stresses will exist in opposition to the force. When heat is applied, the areas of tension and compression shrink at different rates, relaxing the stress in the material and altering the shape.

The first devices fabricated were made at larger sizes (approximately $3 \mathrm{x}$ scale) than the dimensions ultimately needed for the guinea pig backing device. This was done to simplify the fabrication process while inquiry into the process and actuation ability was being performed. Clear heat shrink tubing with a $580 \mu \mathrm{m}$ (0.023") ID and a $20 \mu \mathrm{m}(0.0008$ ") thick wall was used first for this purpose. These $3 \mathrm{x}$ prototypes also loosely approximate the human scala tympani (ST).

After some development of the process, tubing sized for the guinea pig scala tympani was used. This tubing had an inner diameter of $200 \mu \mathrm{m}(0.008 ”)$ and a wall thickness of $4 \mu \mathrm{m}(0.00015 ")$.

\subsection{Fabrication Steps for PET Actuation Chambers}

The following is a step by step account of the basic methods required to fabricate PET actuation chambers found to be most suitable. Each step was developed to be compatible to the requirements of subsequent steps in regards to device geometry, temperature constraints, and bonding methods available.

\subsubsection{Step 1: Pressure inlet port}

The first step is to provide a secure inlet into the tubing that will remain 
open and allow connection of the chamber to a suitable pressure source. A stainless steel hypodermic tube is used for this purpose. The steel hypodermic tube is selected to have a slightly smaller outer diameter (OD) than the inner diameter of the un-shrunk tube, a $50 \mu \mathrm{m}(0.0020$ ") OD clearance fit in this case.

The steel tube is prepared by first cutting a $20 \mathrm{~mm}\left(0.75^{\prime \prime}\right)$ length with a pair of wire snips. Each end is then ground flat using a Dremel tool with a very fine grit sand paper (Buehler ${ }^{\circledR}$ Ltd. 3/0 grit) mounted on a wheel. The edges on each end are also beveled on the sanding wheel to simplify insertion into the PET tubing (Fig. 4.1).

A length of PET tubing is cut from the stock source using a razor sharp pen knife to ensure a clean cut that leaves the tube end open. About $6 \mathrm{~mm}(0.25$ ") of the end of the steel tube is inserted into the end of the PET tubing. A small drop of Dymax 201-CTH UV cure adhesive is applied to the edge of the PET tubing on the steel tube. With slight turning of the steel tube within the PET tube and capillary action, the adhesive moves into the slight gap created by the interface between the two tubes. The tubes are then placed in a UV light chamber for 30 seconds to cure the adhesive. The UV chamber consists of a $100 \mathrm{~W}$ Mercury vapor lamp mounted inside a box with a white reflective interior and a $150 \mathrm{~mm} \times 150 \mathrm{~mm}$ (6" x 6") access port with a dark cloth cover to shield the user from UV radiation.

\subsubsection{Step 2: Chamber flattening}

For building a single actuation chamber, the tube is "flattened" to produce a high aspect ratio cross section necessary for actuation. To accomplish this, a flattening fixture is used. The original fixture consisted of two aluminum plates that could be bolted together. The bottom plate had three $2 \mathrm{~mm}$ wide grooves that are 40, 60 and $80 \mu \mathrm{m}$ deep. The PET portion of the tube assembly was placed 

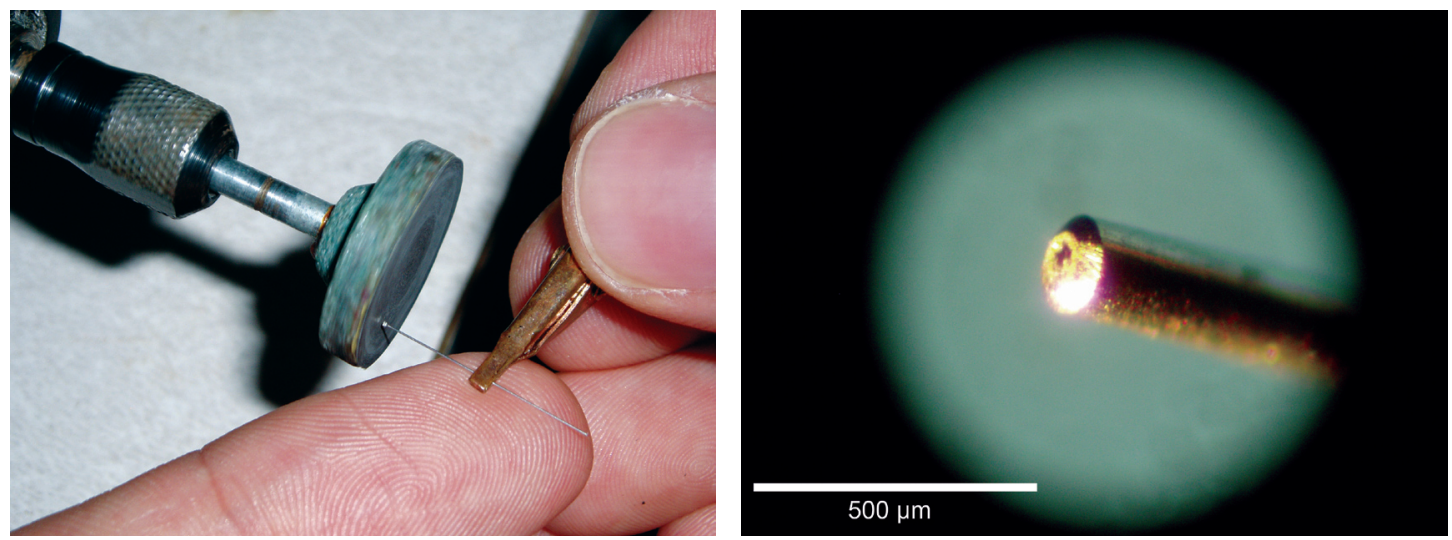

Figure 4.1 Grinding of a steel hypodermic tube and the resulting beveled edge.

Figure 4.2 Transition from round steel hypo to flat PET tubing.

a)

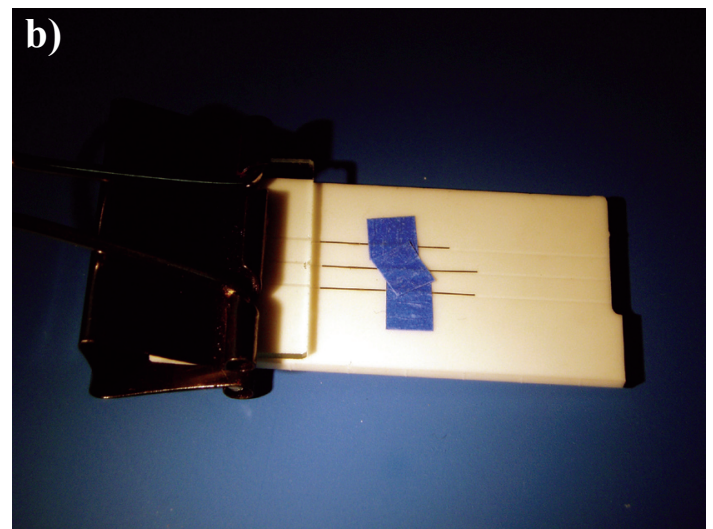

Figure 4.3 a) Macor flattening fixture and b) setup for flattening. 
in one of these grooves and clamped down using the other smooth plate and bolts.

To create a smooth transition between the steel tube end within the PET tube and the flat portion of the PET tube, 1 to $2 \mathrm{~mm}$ of PET tubing remains unclamped. It was found that not having this transition would often lead to a pin hole in the PET tube caused by the edge of the steel tube pressing into it upon thermal shrinkage. A smooth transition from the round portion to the flat portion solved this problem (Fig. 4.2).

Once the tube was firmly clamped, the fixture was placed on a hot plate and the temperature slowly brought up to $70^{\circ} \mathrm{C}$ over approximately 5 minutes. Heating not only locks the tube into a flattened shape but also seals the PET tube firmly around the steel hypodermic inlet.

Temperature was measured using a Type $T$ thermocouple and the reading was taken using an Omega HH506R electronic readout thermometer. The thermocouple wire was inserted into a small hole located in the edge of the upper plate for this purpose. The fixture was removed from heat and allowed to cool to room temperature before opening.

The latest version of the flattening fixture is made from Macor ${ }^{\circledR}$, a machinable glass ceramic. Three identical grooves are machined into the surface that are $250 \mu \mathrm{m}$ wide and $40 \mu \mathrm{m}$ deep (Fig. 4.3a). The plate was machined using a $200 \mu \mathrm{m}(0.008$ ”) diameter two-fluted end-mill on a Dover micromilling machine. Each end of the tube assembly is held flat on the plate with small squares of masking tape and a glass slide serves as the top cover (Fig. 4.3b). The two are clamped together using a black spring paper clip.

The new fixture improves on the aluminum one with smoother surfaces and faster clamping and unclamping. Heating takes place more evenly due to the lower 
conductivity of heat with the material change from metal to ceramic. Experience with the first fixture demonstrated that the $40 \mu \mathrm{m}$ deep groove proved ideal for the smallest sized PET tubes used in the 1x scale backing device. Grooves less than $40 \mu \mathrm{m}$ were found to lead to a condition of "locking up" in the actuation chambers. In a locked chamber, fluid cannot get inside because the inner walls stick from excessive flattening.

\subsubsection{Step 3: Chamber end sealing}

The end of the chambers are sealed shut using a tool that incorporates a 40 gauge $(0.0032 ", 79 \mu \mathrm{m})$ diameter nichrome wire connected to a regulated current source. The nichrome wire is threaded in tension between the eyes of two steel sewing needles held in parallel (Fig. 4.4). Each needle is soldered in place on an etched fiberglass copper circuit board containing two leads which are connected to wires that run along the handle and to the current source.

To seal a tube end, the flattened tube is held flat against a sheet of rubber gasket material. The nichrome wire is placed across the tube at the sealing point with slight pressure into the rubber. The current source is then turned on for approximately 2-3 seconds heating the wire and sealing the PET at the point of contact. A current setting of $280 \mathrm{~mA}$ is used to seal the chamber, while a higher setting will completely melt through the chamber and seal the end. Leaving an additional portion of the chamber still attached beyond the seal is useful for providing a clamping area used in later steps of the manufacturing process.

\subsubsection{Step 4: Chamber bonding and electrode array integration}

Each PET chamber is slightly roughened prior to bonding to the other chambers and the silicon electrode array, which promoted adhesive bonding. Roughening is accomplished by gently rubbing the chamber surface with a small piece 



Figure 4.4 Chamber sealing apparatus and closeup view of nichrome wire.

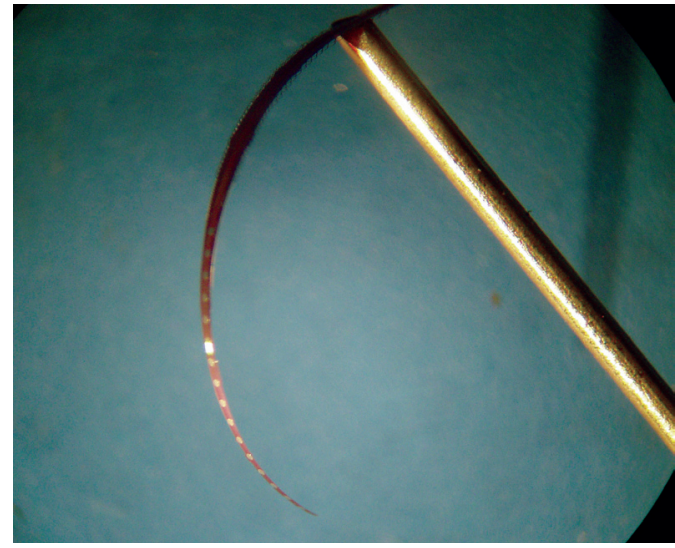

Figure 4.5 Silicon probe with wrongly directed curvature.

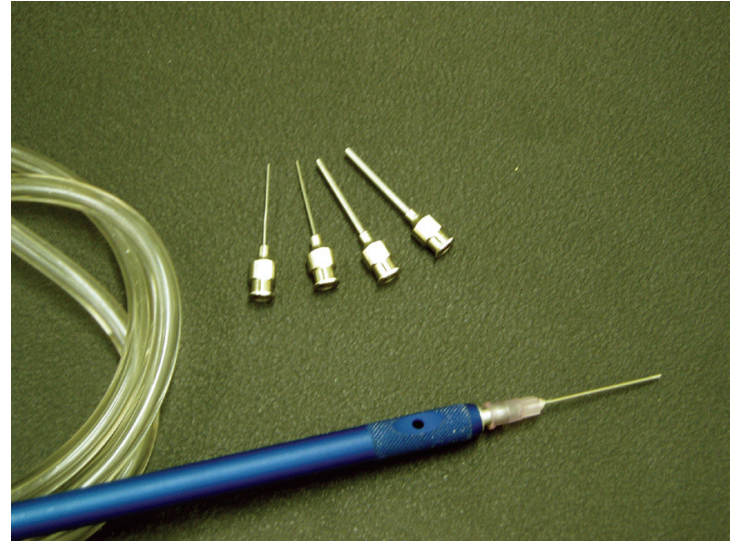

Figure 4.6 Vacuum tweezer set for probe manipulation. 
of Buehler® Ltd. 3/0 grit sand paper. A thin layer of UV cure adhesive (Dymax 201$\mathrm{CTH}$ ) is applied using a single fiber loop from a small synthetic bristled paint brush.

The chambers are first glued together into the stacked configuration one at a time with 1-2 minutes of curing in the UV chamber after the addition of each chamber. UV adhesive is then applied to the backside (opposite the electrodes) of the silicon electrode array.

Previously, due to film stresses in the silicon array, built-in curvature was in the direction opposite the electrodes. In other words, the array had a backward curvature to a modiolar-hugging configuration (Fig. 4.5). This made bonding to the backing device difficult as it was almost impossible to bring the two bonding surfaces into contact so that the surface tension of the adhesive could hold the two pieces together for UV curing.

A vacuum fixture was designed to hold the silicon array flat for the bonding procedure. The vacuum fixture consisted of a brass plate containing 5 in-line holes $(<200 \mu \mathrm{m}$ diameter $)$ that set into an aluminum block with a rubber gasket that has a port to hook to a vacuum pump.

Later versions of the electrode array solved the problem of film stresses and achieved a curvature in the correct direction. These devices could be bonded to the backing by holding the silicon array with vacuum tweezers and the backing with needle tipped tweezers. The use of vacuum for holding the silicon electrode arrays is essential as they are very fragile and will shatter quite easily if mishandled (Fig. 4.6).

When all chambers are bonded to the probe with the UV adhesive, a final cure in the UV light chamber of 5-10 minutes 
is performed to ensure that all the adhesive is thoroughly cured.

\subsubsection{Step 5: Inducing the final helical shape on the implant}

The final perimodiolar shape is given to the device by carefully winding the assembly around a helical metal mandrel. The 3x scale mandrel was made from aluminum while the 1x scale mandrel was machined from brass rod (Fig. 4.7) (Appendix B). The mandrels were machined using dimensions defined by the parametric equations describing the guinea pig ST discussed in section 2.1.2. The helical radius of the mandrel surface is defined by the ST centerline equations minus the semi-minor axis length of the cross section. This corresponds to the dimensions for the wall of the modiolus.

The additional portion of tubing on the longest chamber beyond the sealed end adjacent to the silicon array left from the sealing step is used to clamp the assembly to the mandrel using a water-based white glue. A $1.2 \mathrm{~g}$ weight is then clamped to the steel inlet tube of the same chamber to provide slight tension in the assembly during winding. The implant is then gently wound onto the helical surface of the mandrel and placed in an oven. The oven is heated to $100-110^{\circ} \mathrm{C}$ to lock the PET chambers and probe into the perimodiolar shape.

Once cooled, the adhesive used to clamp the implant to the mandrel is dissolved in a water bath to release it and the additional portion of PET is trimmed using a razor sharp pen knife under a stereoscopic microscope.

\subsubsection{Supplementary Step: Dual diameter chamber}

An additional step may be added between steps 1 and 2 to create a dual diameter chamber, which is necessary for the leading tip of the implant extending into the apical regions of the ST where the width of the device must be less than $210 \mu \mathrm{m}$. 


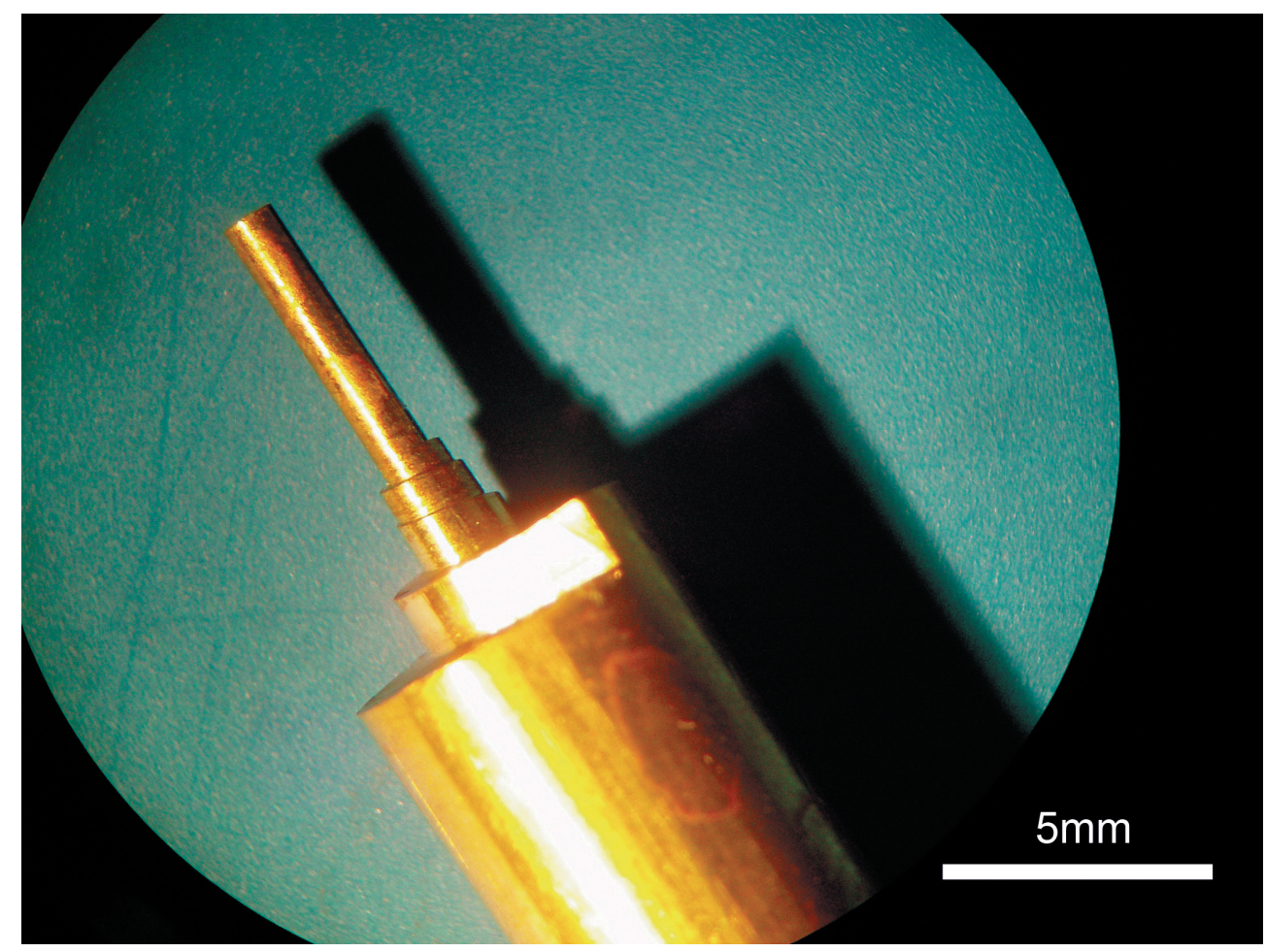

Figure 4.7 Helical 1x brass winding mandrel.

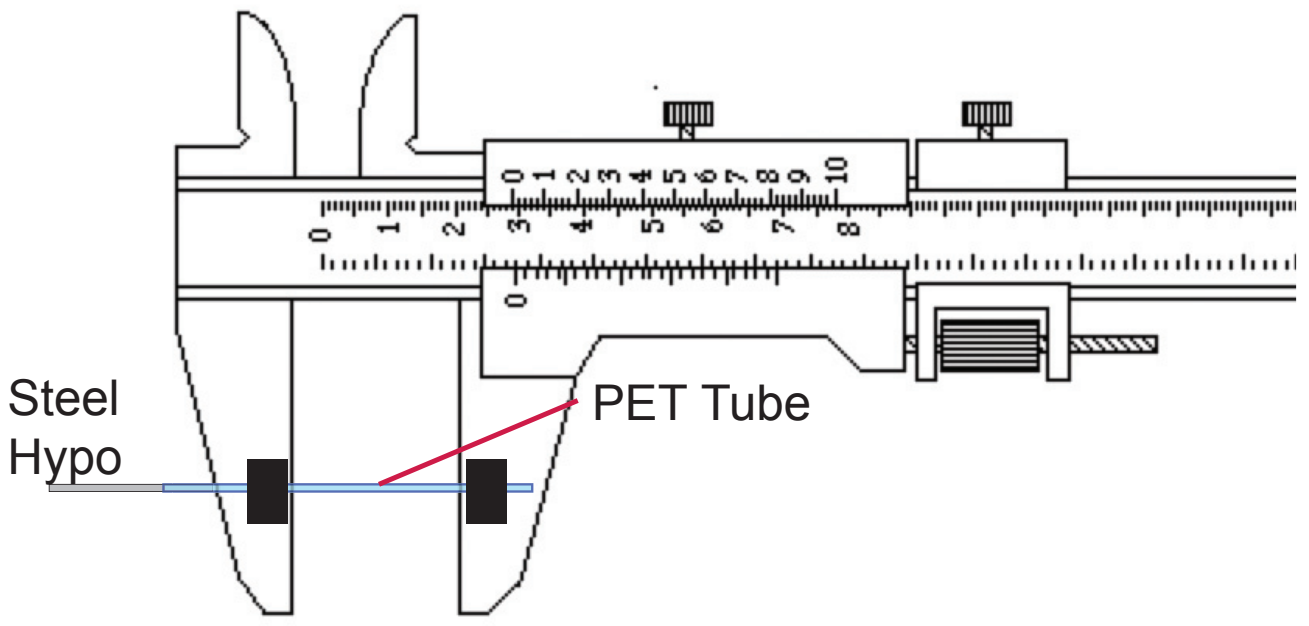

Rubber Clamp Pads

Figure 4.8 Technique for drawing PET tubing to make dual diameter chambers. 
A room temperature drawing technique is used to reduce the diameter of the un-flattened tube down to approximately $160 \mu \mathrm{m}$, which will produce a chamber of $200 \mu \mathrm{m}$ in width after flattening. The portion of the tube to be drawn has each end clamped in a jaw of a vernier caliper. Squares of rubber gasket sheet are used over the tubing to be clamped to protect it from abrasion and damage (Fig. 4.8).

The caliper jaws are slowly moved apart to draw the tube. During the draw, portions of the tubing will begin to neck and reduce in diameter at random along the tube. To fully draw out the tube section so that no un-drawn pockets remain, it was found that increasing the length by approximately $100 \%$ produces a good result. The dual diameter tube can then be removed from the calipers and flattened as normal. Figure 4.9 shows the result of the tube drawing and flattening.

\subsection{Backing Prototypes}

During the development of the insertion tool, a series of prototypes were fabricated for testing of actuation and insertion ability. As fabrication techniques improved, new features were added to address shortcomings of previous devices.

\subsubsection{Early single chamber $3 x$ insertion tools}

As mentioned earlier, the first prototypes were made using larger-sized PET

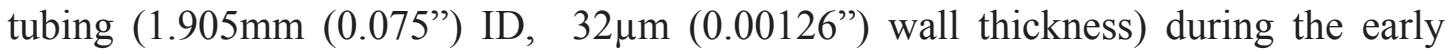
developmental stages of the fabrication process. Figure 4.10a illustrates one of these

early tools in front of the 3x scale guinea pig cochlea ST cavity. The device has 2.5 helical turns and approximates the size of a human cochlea. However, the tool was made before the winding mandrels were machined, meaning that the helix does not have the shape of the guinea pig ST but rather that of a regular, cylindrical helix. Also, the end was sealed using a polymer plug that was locked into the tube end during the shrinking 


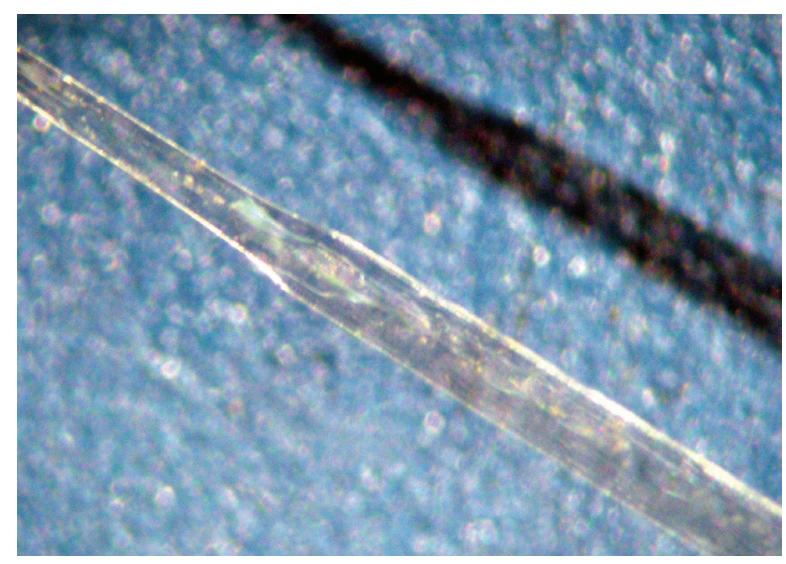

Figure 4.9 A dual diameter PET chamber fabricated by drawing.
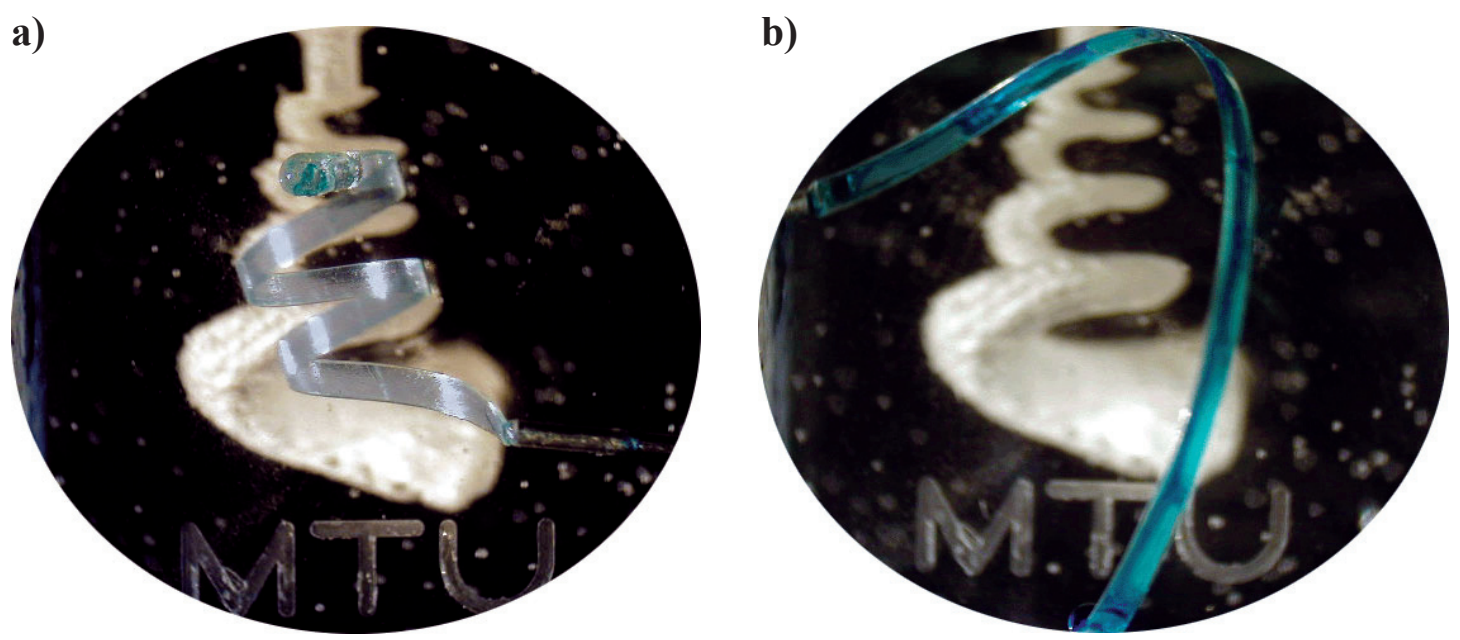

Figure 4.10 a) An early 3x insertion tool and b) its actuation ability .
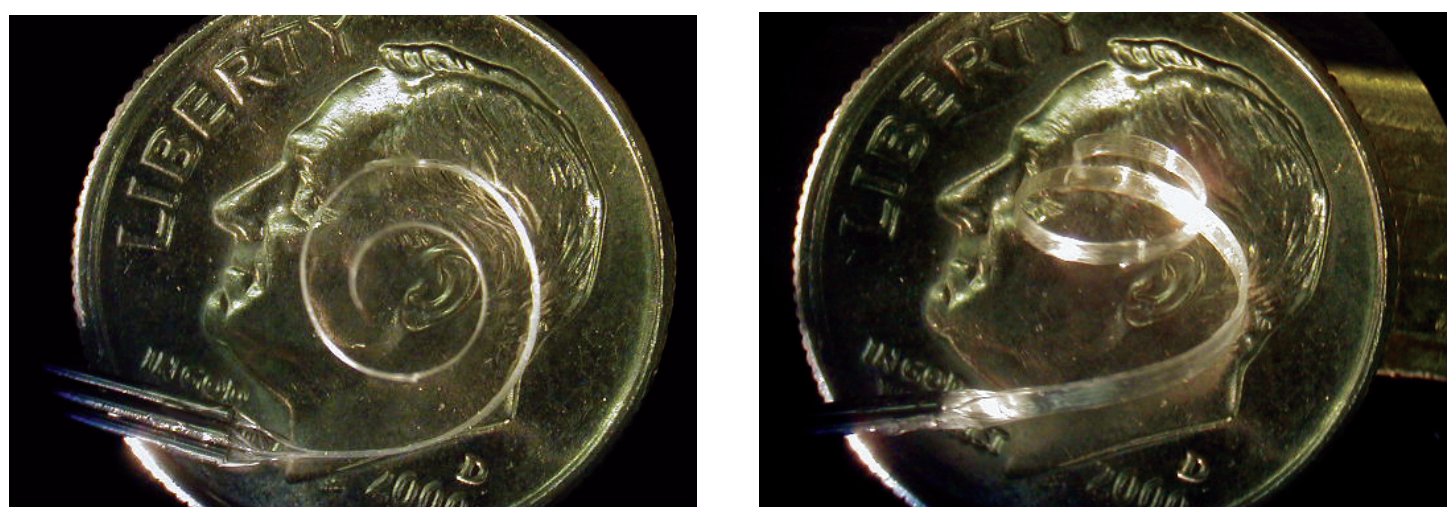

Figure 4.11 Two views of a 3x, three chambered insertion tool. 
process. The plug end seal performs well, but its large size is less than desirable.

The tool had excellent actuation ability (Fig 4.10b). Springback potential was also good since the thicker tube walls experienced less plastic deformation during pressurization than the $1 \mathrm{x}$ thin-walled tubes later used. The device was actuated by hand using a syringe plunger with water that was dyed blue as the fluid medium.

\subsubsection{Multiple chamber $3 \mathrm{x}$ insertion tools}

The next series of insertion devices investigated using multiple chambers in the stacked configuration. Figure 4.11 shows two views of a $3 \mathrm{x}$ guinea pig device fabricated with three chambers pictured in front of a U.S. dime. The two stacked chambers run roughly two-thirds and one-third of the length of the base chamber. Fabrication was accomplished using the steps outlined earlier in this chapter, but the tube ends were sealed using the hot tip of a soldering iron rather than with the nichrome wire tool.

Actuation of the three chambers was accomplished using 3 separate diaphragm type pressure regulators attached to an in-house pneumatic pressure source. The pressure regulating system illustrated in Figure 4.12a provided a different pressure setting for each chamber. Pressure was set using the dials and gauge readings and then actuation could be initiated in each chamber separately by pressing the brass push button valves located at the top of the regulators. A vacuum source could also be attached to the system and each chamber could be connected to this source by pressing three additional push button valves located at the back of the regulating system. Figure $4.12 \mathrm{~b}$ shows the connection between the regulating source and the insertion tool.

With the regulating system, the three chambers were tested to observe the behavior of the stacked chamber design. In Figure 4.13, a series of photographs 
was taken as each chamber was individually pressurized. The pressures in each chamber ranged between 30-40psi $(207-276 \mathrm{kPa})$. The test showed that the successive pressurization of each chamber in the stacked design did indeed provide additional actuation of the tool in the localized region of each chamber's influence. However, it was difficult to control and predict curvature of the device along its length and while the upper chambers in the stack did provide additional actuation, primary actuation was imparted by the full-length base chamber.

\subsubsection{Integration of the insertion tool and silicon electrode array}

Further work led to the integration of the insertion tool with the silicon high density electrode array and then miniaturization of the components down to the $1 \mathrm{x}$ scale. In Figure 4.14, a single chamber has been integrated with a silicon electrode array at the $3 \mathrm{x}$ scale. This silicon array was manufactured at human cochlea dimensions and had a length of $45 \mathrm{~mm}$. The chamber on the back was fabricated using a $200 \mu \mathrm{m}$ ID white pigmented tube with a $4 \mu \mathrm{m}$ wall thickness.

Although the backed electrode array actuated normally, the residual stress of bending the flat silicon probe into a helical configuration increased the radius of the backed device upon releasing it from the mandrel. The relaxing of the device curvature was small however, and was compensated for by decreasing the radius of the winding mandrel.

Another concern associated with backing integration to the electrode array is the fragility of the silicon substrate in the array. The silicon probe exhibits excellent flexibility when bending is in the direction of its thickness. But, the probe shatters when minimal force is applied in the other directions or as a point contact force on its surface.

For winding on the mandrel, where the probe face comes into contact 

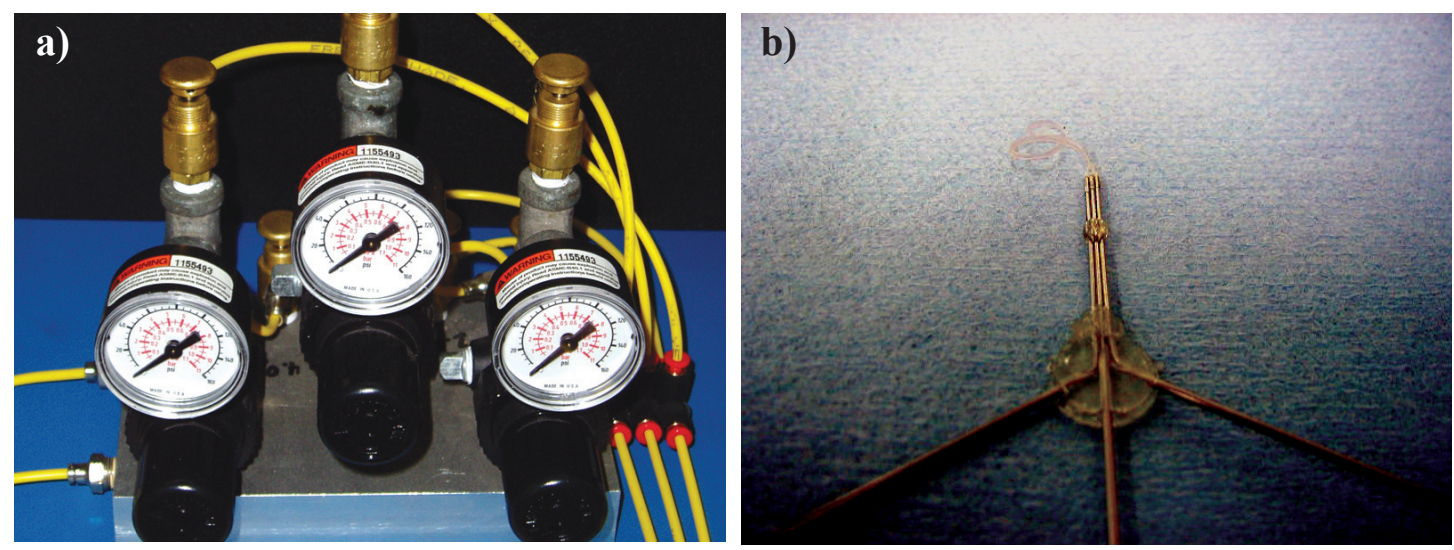

Figure 4.12 a) Pressure regulating system and b) attached to a three chambered prototype.
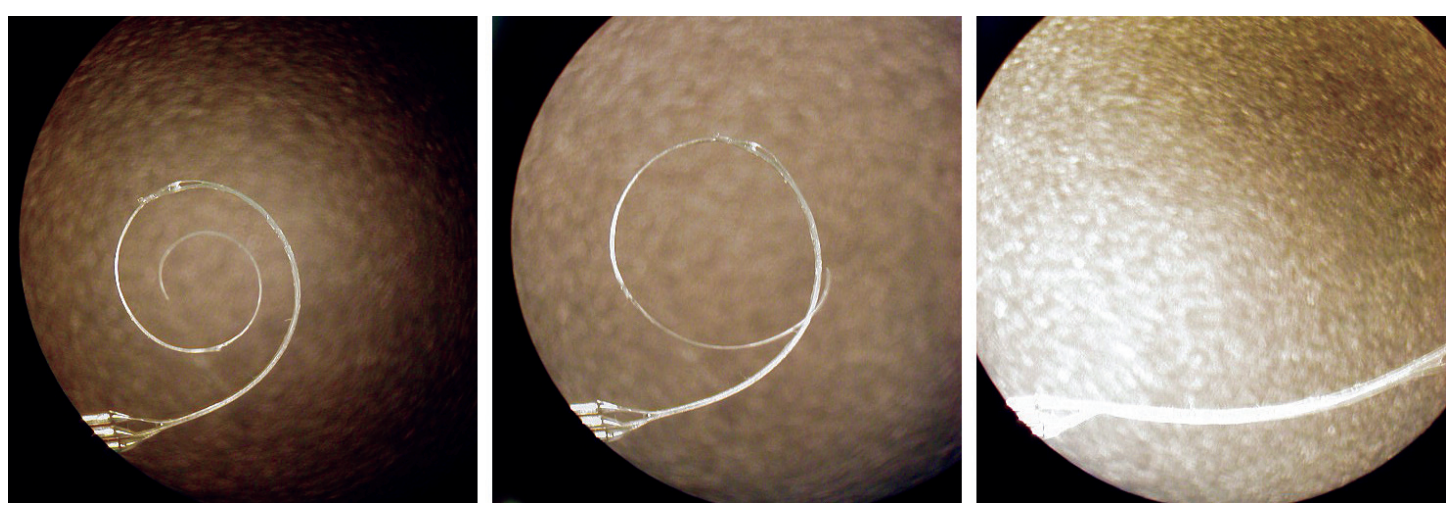

Figure 4.13 Actuation of a three chambered insertion tool with successive pressurization of each chamber to 30-40psi.
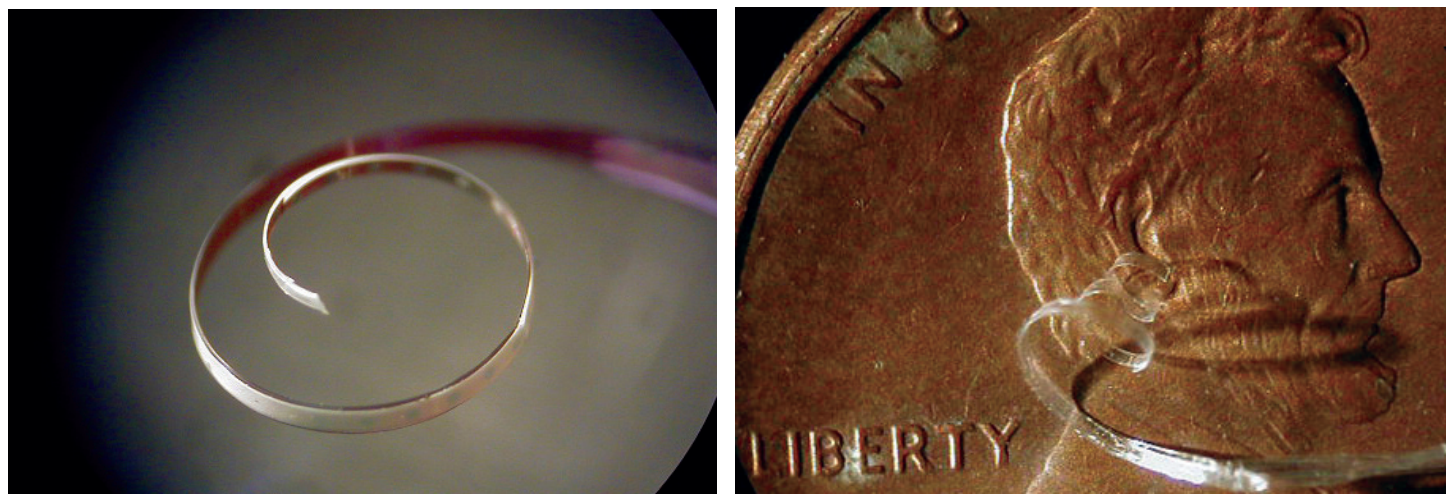

Figure 4.14 A 3x single chambered insertion tool with electrode array. 84
Figure 4.15 A 1x three chambered insertion tool. 
with the mandrel surface, it was necessary to have a very smooth surface on the mandrel devoid of any pitting or marks. Coating the mandrel with a thin layer of silicone is another method that was found to work. A disadvantage with this method though, is that after several uses the silicone layer needs to be stripped and reapplied when it begins to disintegrate from use.

Figure 4.15 illustrates a $1 \mathrm{x}$ three-chambered device manufactured using clear $200 \mu \mathrm{m}$ ID tubing with $4 \mu \mathrm{m}$ thick walls. The device has 2.5 turns and is sized to fit within the guinea pig ST. A major challenge for fabrication at this size was actuation chamber lock-up. Inducing the small radius of curvature into the chambers that is required at the $1 \mathrm{x}$ scale often caused the actuation ability of the chamber to "lock-up" as described earlier in Chapter 3. The problem was solved by adjusting the channel depth in the Macor flattening fixture to avoid the inner walls of the chamber from touching upon curling on the mandrel. A channel depth of $40 \mu \mathrm{m}$ provided a suitable compromise between flattening ability and avoidance of lock-up.

The device pictured in Figure 4.16 is a $1 \mathrm{x}$ scale single-chambered with 1.25 helical turns. The device actuates correctly, but lacks a modiolus hugging shape. The curvature is small enough to fit the guinea pig cochlea but its shape puts it into contact with the outer wall away from the modiolus.

Difficulty arose in achieving the modiolus hugging shape partly because of the stiffness of the silicon probe. Although the thickness of the silicon probe is only 4$5 \mu \mathrm{m}$, the large modulus of elasticity of silicon compared to that of the PET chamber causes its stiffness to be 25-30 times that of the backing chamber. As a consequence, the curling radius of the mandrel must be significantly smaller than the desired curvature at the $1 \mathrm{x}$ scale. Attempting to wind the backed probe around a mandrel of 


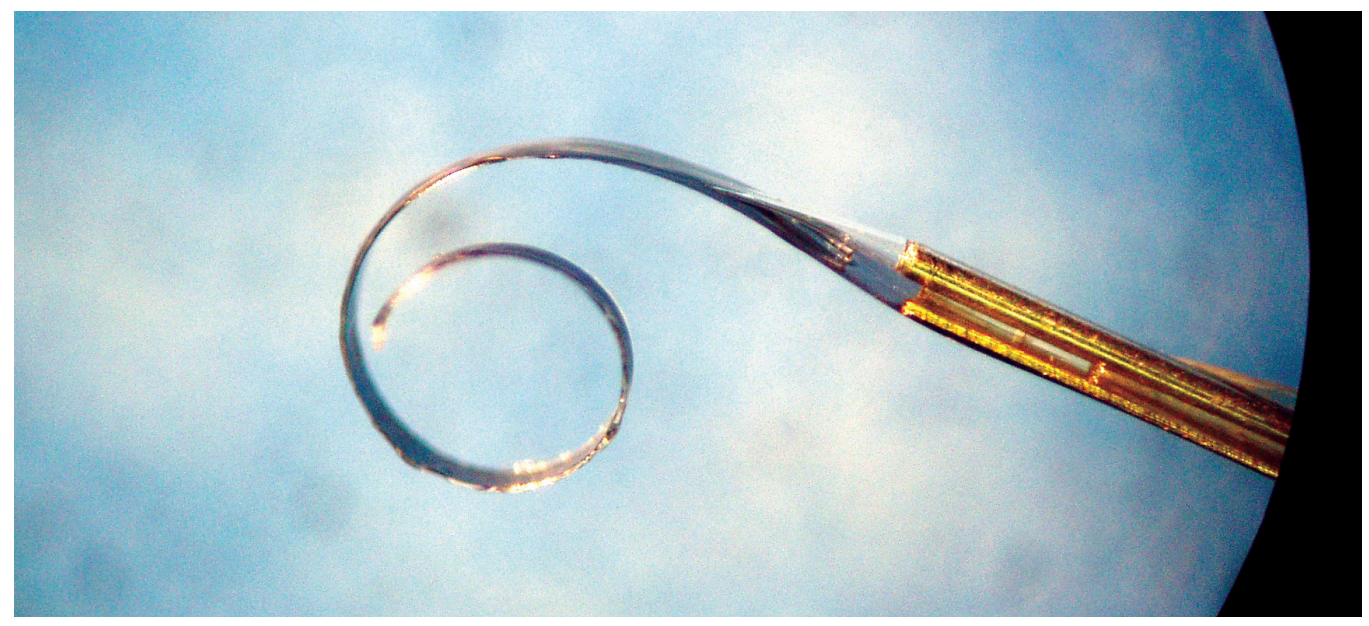

Figure 4.16 A 1x insertion device integrated with a silicon electrode array and protective brass backing.

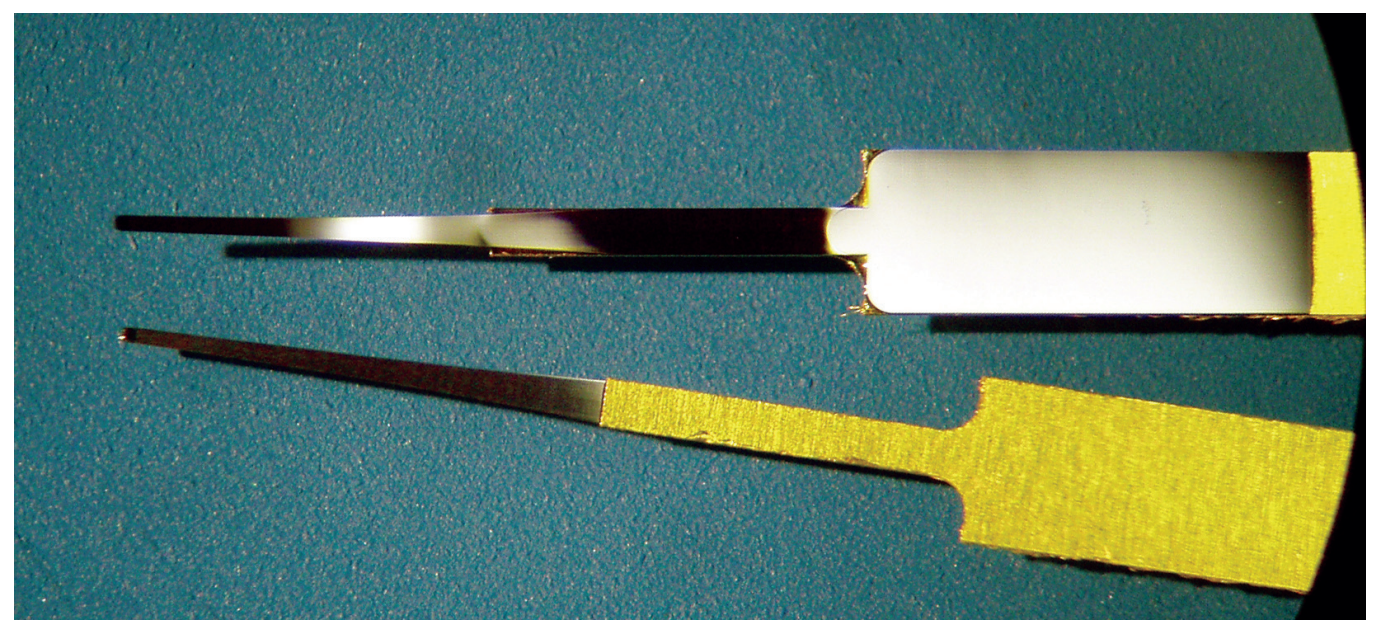

Figure 4.17 Protective brass backing attached to a silicon electrode array. 
this curvature $(\sim 500 \mu \mathrm{m}$ OD) exceeded the flexibility of the silicon leading to fracture.

In addition to the curling problem, many of the silicon probes retained portions of the sacrificial layer used to release the probes from the wafer after fabrication. The sacrificial layer is attacked chemically from the edges and moves inward dissolving the layer to release the probes from the wafer surface. On most probes, the layer remained intact on the back end and on the shank of the probe up to the taper where it formed a point. The area at the point created a region of stress concentration, which led to an almost complete loss of probes during winding onto the mandrel.

The problem was temporarily overcome by attaching a brass foil backing to protect the probe during the winding process (Fig. 4.17). The $5 \mu \mathrm{m}$ thick foil was machined to have the shape of the back end and $3 \mathrm{~mm}$ of the shank portion of the probe (Appendix B). The brass back was attached to the probe using a low melting temperature hard bonding wax.

A permanent solution to both the curling radius and sacrificial layer problem came by altering the production process of the probe at the University of Michigan, Ann Arbor. The process was altered to change the film stresses in the probe causing the curvature of the probe bend in the correct direction. Wafer release procedures were also modified to assure that no sacrificial layer remained after processing.

With the curvature of the probes adjusted to the correct direction, the springback force in the probe was reduced enough to produce modiolus hugging insertion tools. In Figure 4.18 a 1x insertion tool attached to a silicon electrode array is shown above the 2D guinea pig ST cavity. The device has 1.25 turns and a radius that closely hugs the modiolus. The chamber was fabricated using the tube drawing method to decrease 
the width of the tip portion of the chamber to match the width of the electrode array.

\subsection{Insertion testing of prototypes}

Several insertion procedures were performed at various scales and in different cochlea models to test the insertion ability of devices with a variety attributes. This section presents an early $3 \mathrm{x}$ test done by Butala and later testing performed at the $1 \mathrm{x}$ scale.

\subsubsection{3x scale insertion tests [32]}

Performance tests were done on the insertion tools using the acrylic cavities described in Chapter 2. The 3x scale tests were done by Butala using a three chambered insertion tool and the $3 \mathrm{x}$ sized ST cavity [30]. The test procedure had the insertion tool prototype mounted in a vise while the three chambers were pressurized to $300 \mathrm{kPa}, 220 \mathrm{kPa}$, and $170 \mathrm{kPa}$ for the base to the top chamber in the stack respectively. The pressurization was adequate to straighten the prototype in preparation for the insertion into the ST cavity. The ST cavity was then manipulated by hand while pressure was reduced in the chambers, starting with the base chamber, so that the prototype curvature would match the ST cavity.

Figure 4.19 shows an insertion test in progress. The test was successful for an insertion of about 1 to 1.5 turns into the cavity. Butala reported that further insertion was hindered due to the overly rough interior surface of the cavity.

\subsubsection{1x scale insertion tests}

Later, several successful insertion tests were performed at the $1 \mathrm{x}$ scale using the two-dimensional 1x ST cavities. For these tests, the cavity was held in a vise and positioned under the stereo microscope for viewing. A singlechamber insertion tool with a drawn tip portion was fabricated to a modiolus 

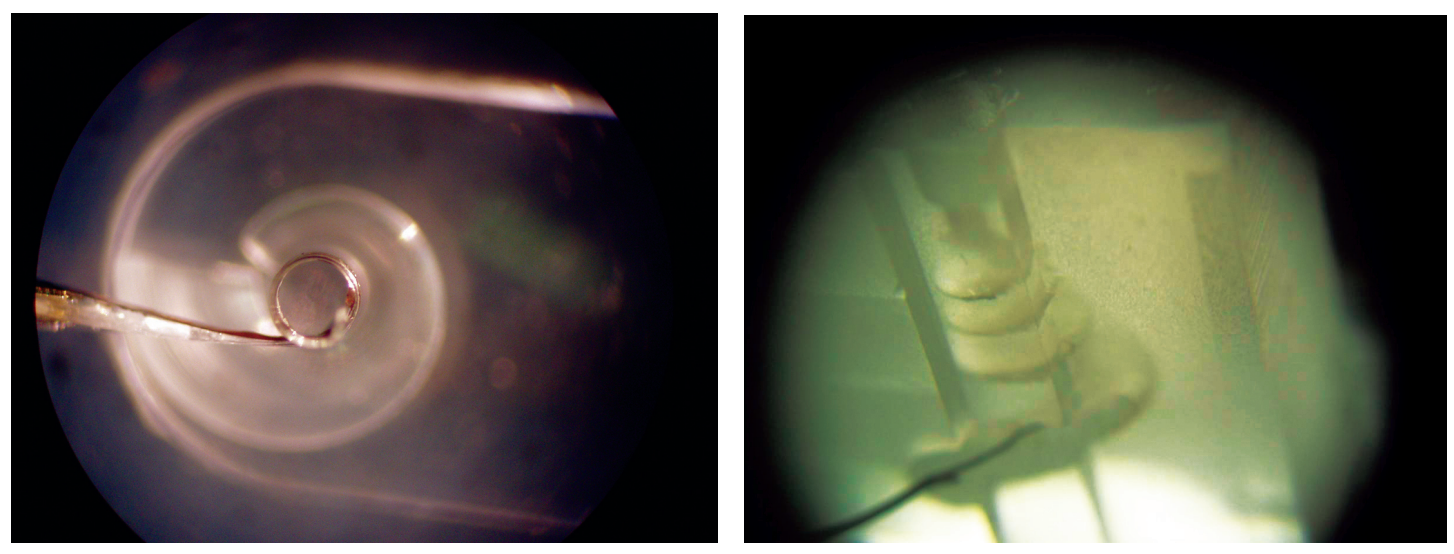

Figure 4.18 Modiolus hugging inser- $\quad$ Figure 4.19 Insertion test for a tion tool integrated with an electrode array shown above 1x ST model. 3x single-chambered device into an acrylic ST model [32].
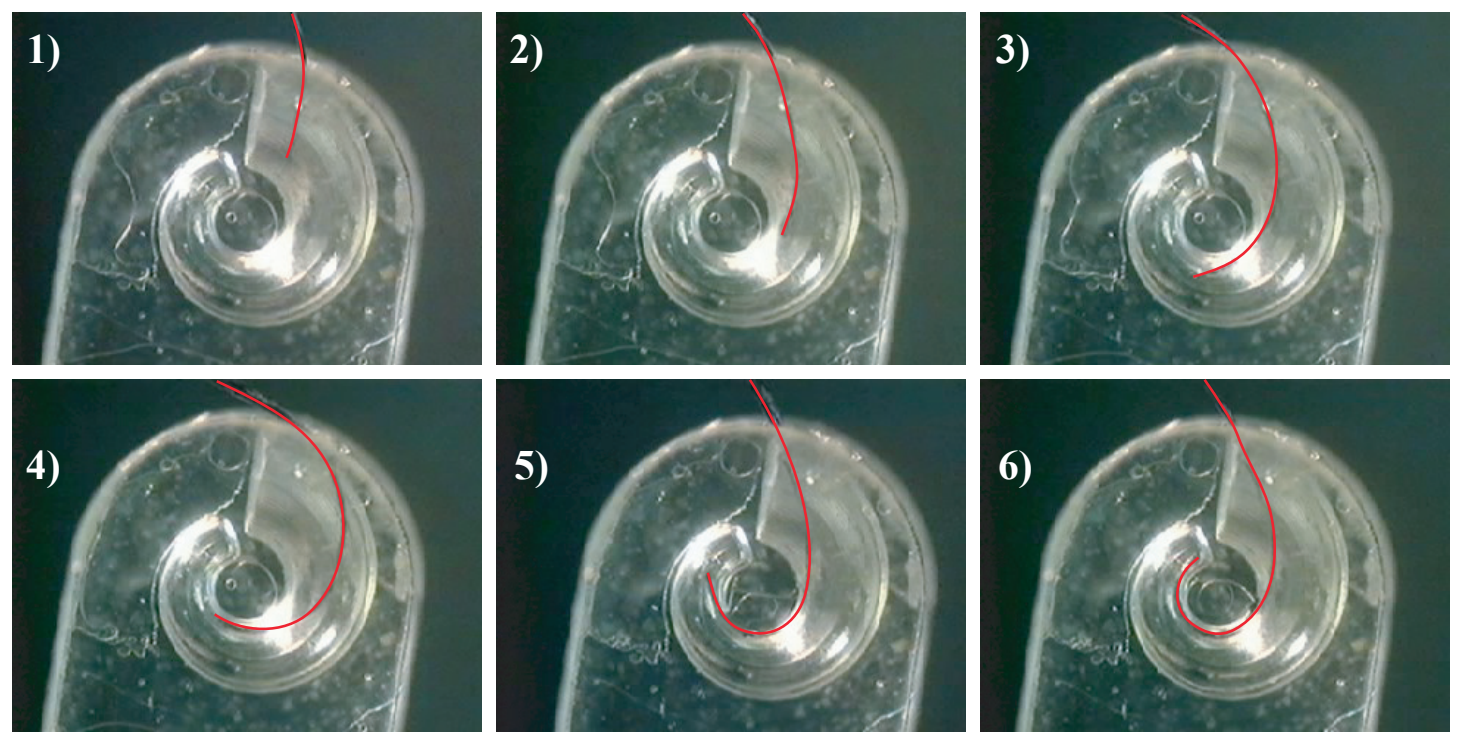

Figure 4.20 A series of photographs from an insertion test for a 1x scale tool. 
hugging shape and mounted to a small $\mathrm{X}-\mathrm{Y}$ stage to facilitate manipulation. Black ink was used to color the edge of the tool closest to the microscope to enhance visibility. The pressure was provided by the in-house pneumatic pressure source and regulated by a single diaphragm type pressure regulator.

The tool was pressurized to a nearly straight curvature and positioned so that the tip was aligned with the opening to the 1x ST cavity. The lead screw controls of the $\mathrm{X}-\mathrm{Y}$ stage moved the tool into the cavity while pressure was reduced to match the curvature of the tool to the cavity curvature. A charge coupled device (CCD) video camera attached to the microscope recorded the insertion procedure, which took approximately 4 minutes to complete. Figure 4.20 shows a series of six images from the recording with the tool highlighted to enhance its visibility in the photographs.

The insertion tests helped to confirm the viability of using this type of actuation to facilitate an insertion of an electrode array into the cochlea. This test was performed in a minimal amount of time (2-3min) and on the first attempt. The tool reached the furthest extent of the cavity and achieved a position adjacent to the modiolus.

Subsequent tests achieved similar results. One test was performed using a silicon probe backed with a single chamber. Figure 4.21 shows the tool's final position within the ST cavity. The tool reached close to the end of the cavity, but because the tool lacked the modiolus hugging curvature, it achieved a position towards the cavity's outer wall. The tool lacked the modiolus hugging shape due to the probe curvature and stiffness as mentioned earlier in section 4.3.3. 


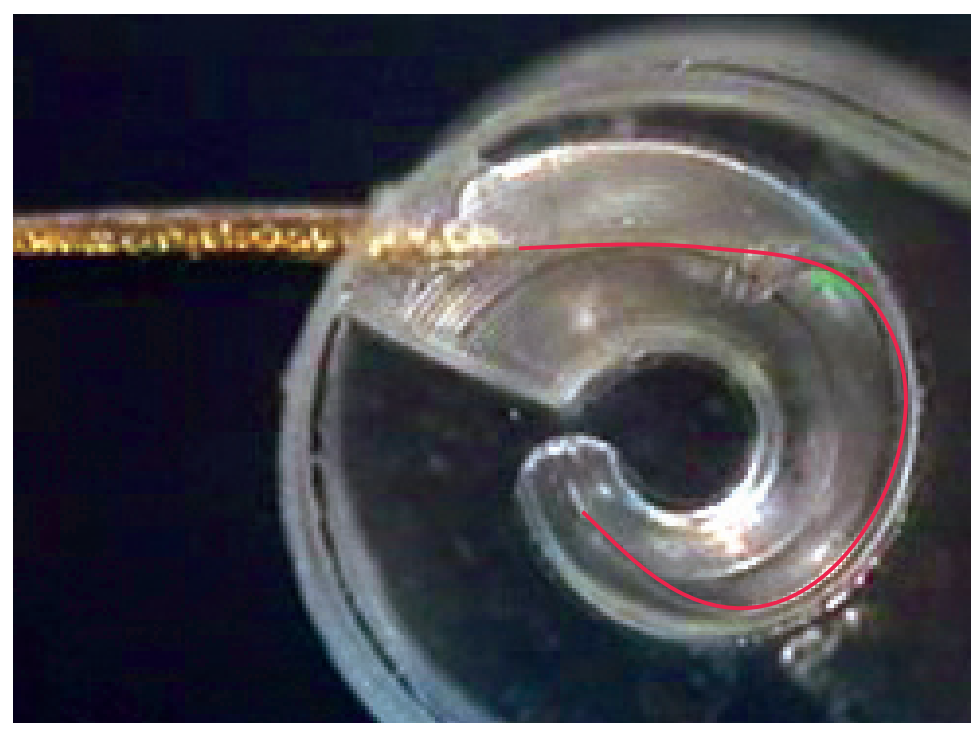

Figure 4.21 Insertion test for a 1x device with an integrated silicon electrode array.

\subsubsection{Integration of electrokinetic pumps}

While the insertion tests demonstrated that the actuation concept for cochlear implantation is valid, they highlighted issues that needed to be addressed. The success of the insertion tests was aided significantly by the visibility of the tool within the clear cavity. An actual insertion into the cochlea would be done without the advantage of seeing where the inserted portion of the implant is located. This observation underscores the need for a computer controlled insertion system that uses feedback from the sensors located on the silicon electrode array to determine the position of the device within the cochlea.

The diaphragm type pressure regulators used to control the actuation of the chambers have performed well enough for testing purposes, but there are significant drawbacks for their use in an insertion tool system. First, they are large and bulky in comparison to the chambers making the connections between the two components unnecessarily complicated and cumbersome. Control of the pressure 
with the regulators must be done manually and is imprecise. To move toward a computer controlled insertion system, an improved pressure source was needed.

Electrokinetic (EK) pumps have marked advantages over the diaphragm type regulators for application to the insertion tool. They can be built to very small sizes, small enough to become part of the insertion tool itself. They can use an incompressible buffered ion solution as the pumping medium, which can be biocompatible (saline solution). Finally, EK pump pressure is conveniently controlled by the magnitude of the supplied DC voltage making them an ideal choice for a computer controlled feedback system.

Electrokinetic pumps make use of the principle of electroosmotic pumping. If the walls of a microchannel have an electric charge, as most surfaces do, an electric double layer of counter ions will form at the walls. The double layer is attributed to a layer from the wall material and another from the fluid. When an electric field is applied along the channel, the ions in the double layer move toward the electrode of opposite polarity. This creates motion of the fluid near the walls and transfers, via viscous forces, into convective motion of the bulk fluid (Fig. 4.22) [32, 33].

In his Masters work, Sudeep Shyamsunder [32] examined the applicability of EK pumps as the pressure source for the insertion tool. The first EK pump studied was designed and built at the University of Michigan's Microfluidics Laboratory in Ann Arbor. It consisted of five poly-methyl-methacrylate (PMMA) disks and a sintered silica pumping disk containing the necessary chambers and components. When assembled, it measured $53 \mathrm{~mm}$ in length and had a diameter of $31 \mathrm{~mm}$ (Fig. 4.23).

The center disk held an $11 \mathrm{~mm}$ diameter silica disk with a nominal 




Figure 4.22 Diagram of the principle of electroosmotic flow.

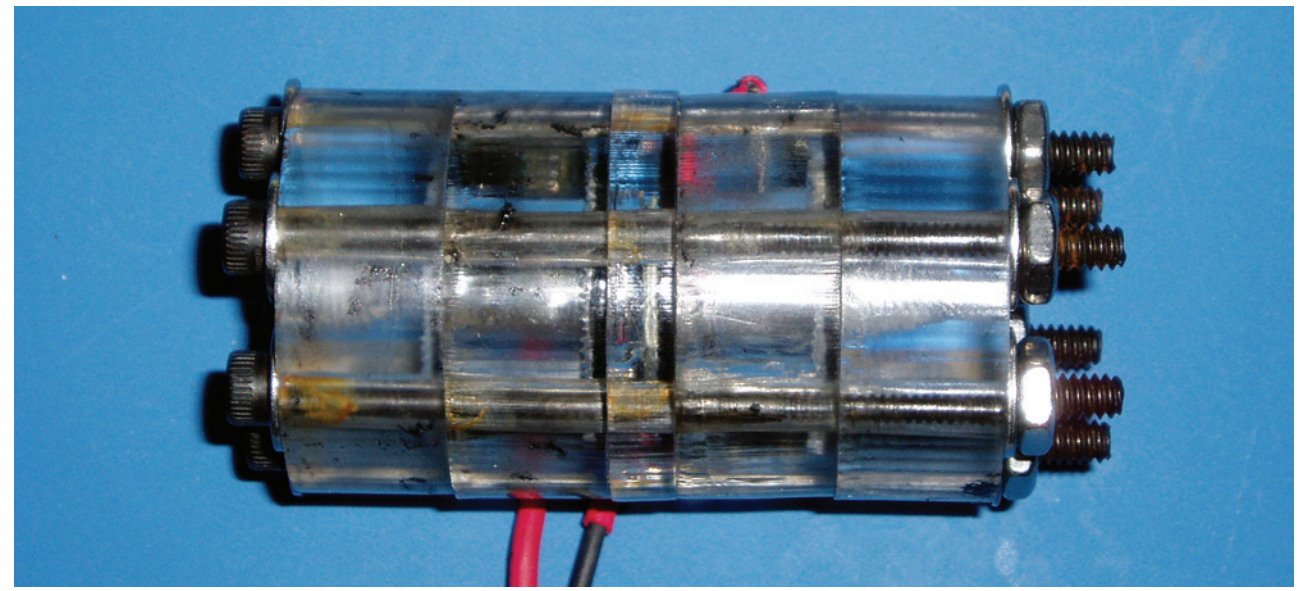

Figure 4.23 Electrokinetic pump developed at the University of Michigan Microfluidics Lab. 
$200 \mathrm{~nm}$ pore size. Pressure and flow is developed in the channels created by the porosity of the disk. Pumping pressure and flow rate are strongly dependent on the size and number of channels. The two adjacent PMMA disks contain an $11 \mathrm{~mm}$ diameter bore used as a fluid well and platinum wires used as electrodes. The outer disks have threaded bores used for fluidic connections.

For operation, the assembly is bolted together and the fluid wells are filled with a $1 \mathrm{mM}, 7.7 \mathrm{pH}$ buffer solution and the platinum wires are connected to a voltage source (Protek 3006B DC Power Supply with a 0-60V range). To attach an insertion tool to the pump, a nut and ferrule fitting with a polyether-etherketone PEEK tubing sleeve was used. The PEEK tube had a 1/16" $(1.587 \mathrm{~mm})$ OD and a $0.015 \%(381 \mu \mathrm{m}) \mathrm{ID}$, in which a steel inlet hypodermic tube from the insertion tool could be inserted with adhesive (for sealing).

The EK pump operation has an almost linear relationship between applied voltage and the resulting flow rate and pressure. Tests performed at the Microfluidics Lab resulted in the performance envelope for flow and pressure shown in Figure 4.24.

With the University of Michigan's EK pump connected to a single-chambered backing device, tests were run to determine if the pump would have an adequate flow rate to actuate the chamber in a timely manner. The procedure entailed applying a voltage to the pump and observing the time taken to achieve the maximum actuation of the chamber. The results of the experiment are shown in plot in Figure 4.25. The actuation time tests indicate that at low voltages a maximum of 150 seconds were required and less than 20 seconds at high voltages. With this approximate relationship, these preliminary tests indicate that automatic control should be achievable as there is a monotonic and well-behaved relationship between pump voltage and chamber response [32,34]. 
Pmax-Voltage

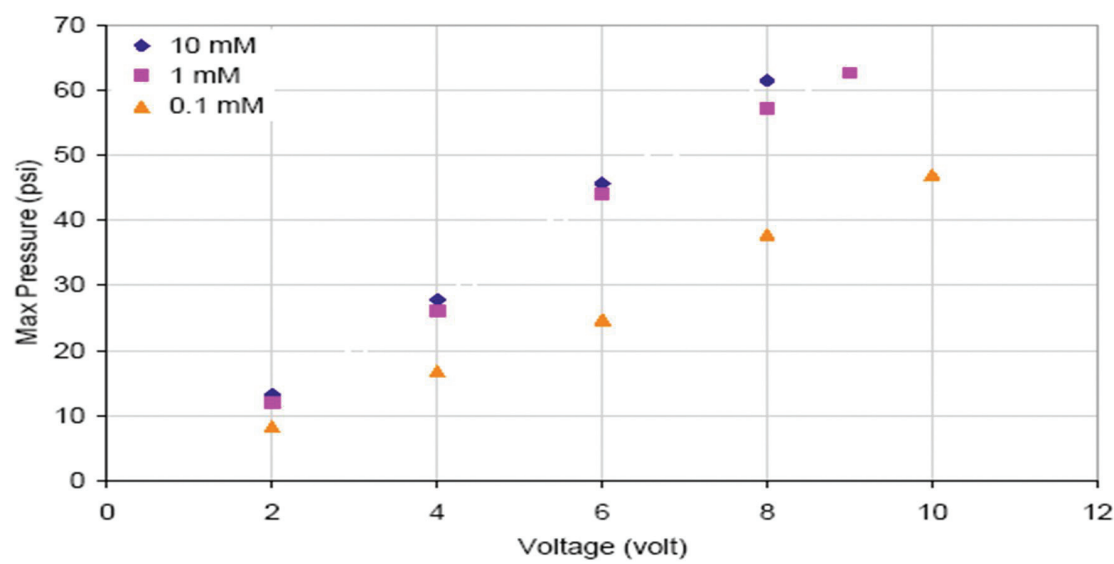

Flowrate-Voltage

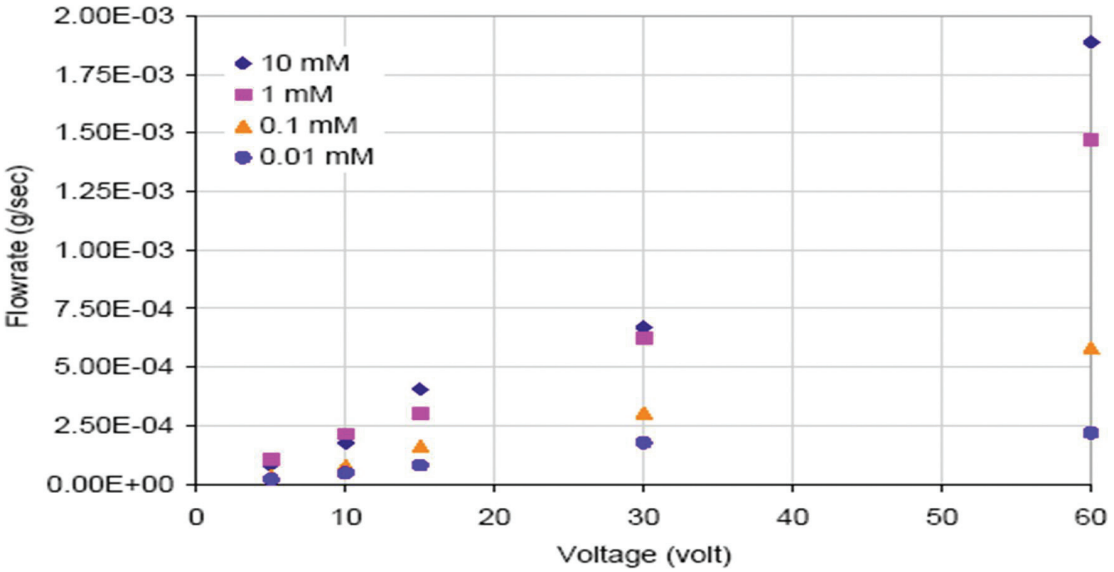

Figure 4.24 Performance plots for the UofM EK pump [32].

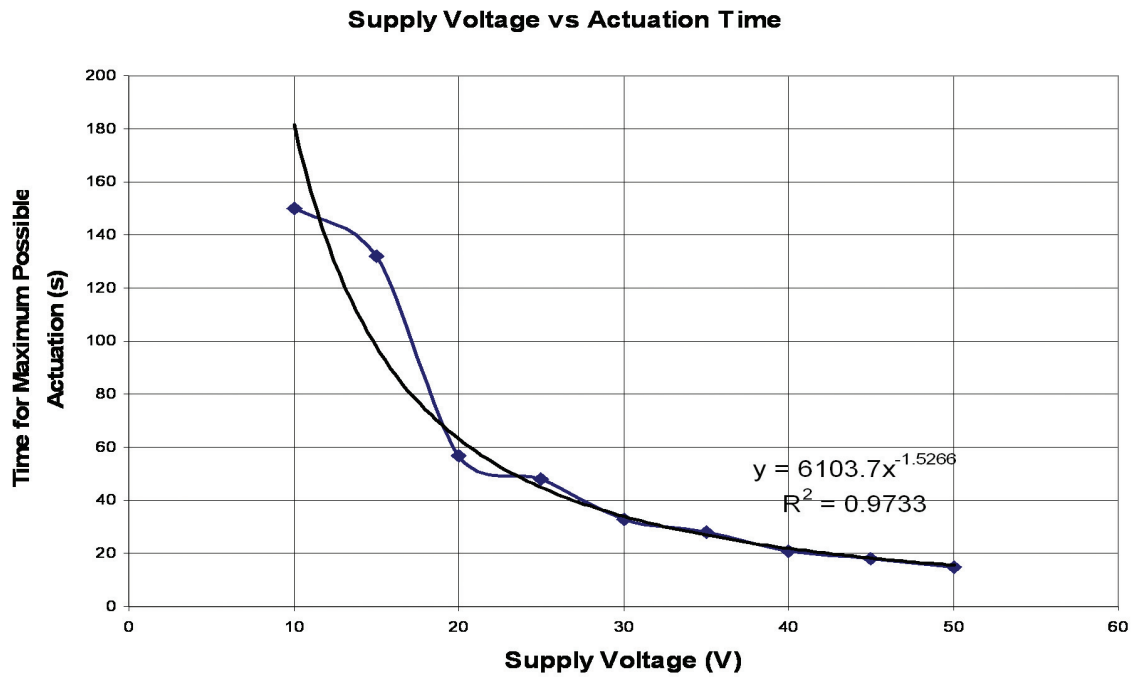

Figure 4.25 Actuation time of a single-chamber tool using the UofM EK pump [32]. 

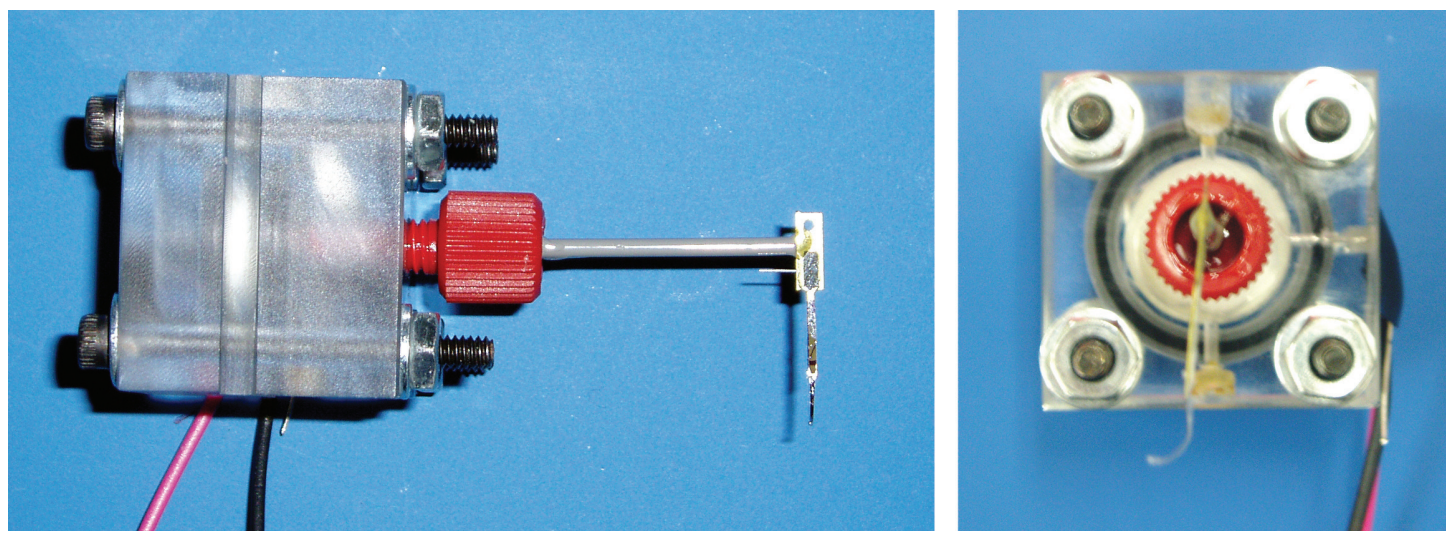

Figure 4.26 Redesigned EK pump with attached single chamber insertion tool and electrode array.

Supply Voltage vs Actuation Time: A Comparison

$\leadsto$ - Old Pump $\quad-$-Redesigned Pump No.1

$\leftarrow$ Redesigned Pump No.2 - $\leftarrow$ Redesigned Pump No.3

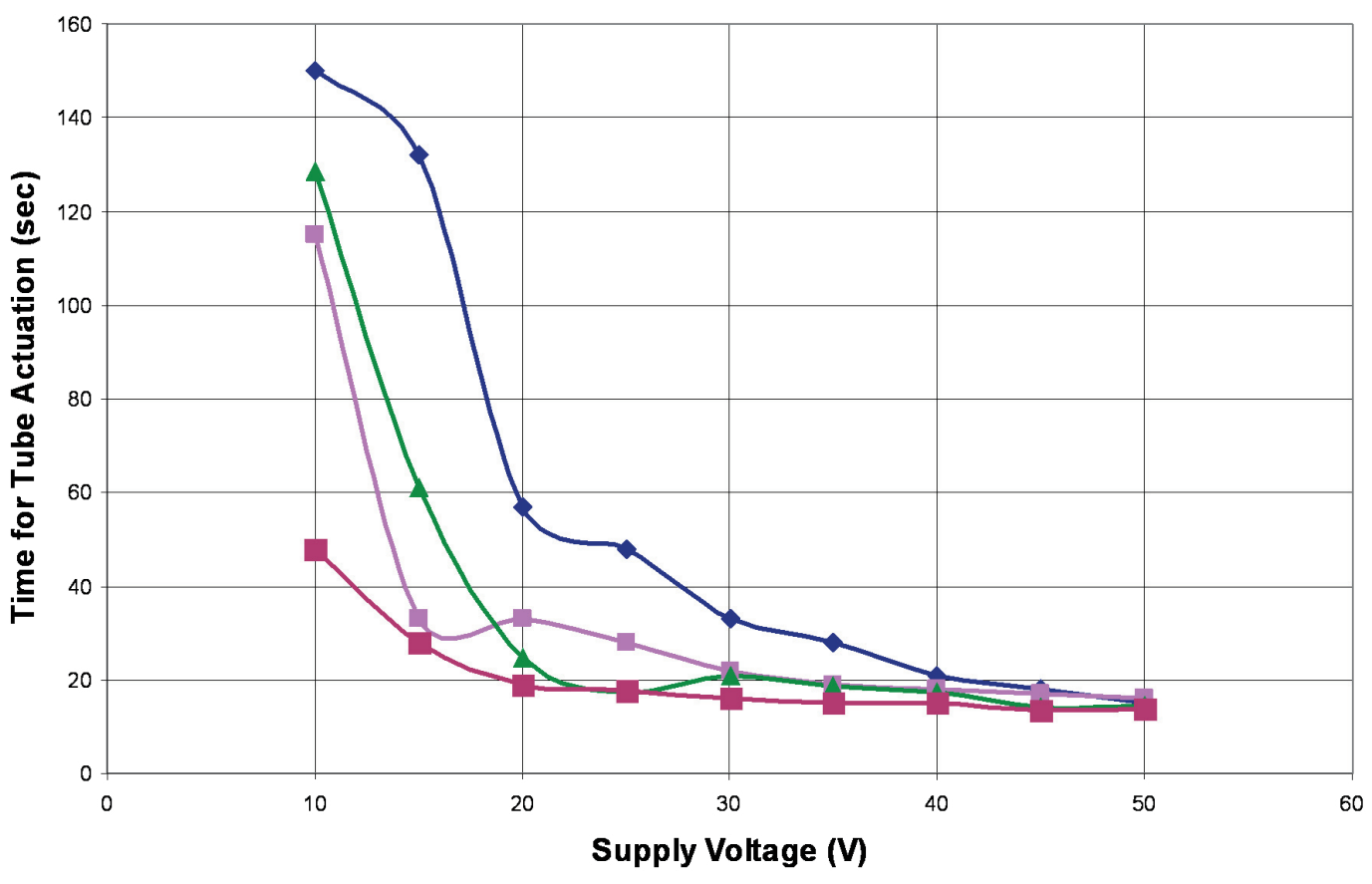

Figure 4.27 Actuation times of a single chamber insertion tool for the redesigned EK pumps [32]. 
The University of Michigan (UofM) EK pump was never intended to be integrated with the insertion tools, rather it was a starting point to lay the groundwork and establish design criteria critical for application to the insertion tool. With the knowledge that EK pumps could indeed be a valid alternative to the pneumatic pressure source used to date, Shyamsunder began to develop a design better suited to integration with the insertion tool. The design, while conceptually similar to that of the UofM EK pump, consisted of three PMMA sections with the center section holding an $11 \mathrm{~mm}$ diameter porous silica disk. In all, three of these redesigned EK pumps were fabricated, each having overall dimensions of a cube $26 \mathrm{~mm}$ on each edge (Fig 4.26).

The performance of the new EK pumps in terms of actuation time of an insertion tool was an improvement over the UofM design. Figure 4.27 shows a plot of the actuation times for the three redesigned pumps against the UofM design for applied voltages. Power consumption for the new pumps was about $200 \%$ higher than that of the UofM design, but overall was still low at about $550 \mathrm{~mW}$ when operating at $50 \mathrm{~V}$. 


\subsection{Next Generation Fluidic Actuator Manufacturing Process}

Handling of the components during fabrication becomes a primary consideration at the scale of the insertion tool. Many of the processes need the aid of a microscope to be performed consistently and successfully. Contamination by dust and skin oils becomes a major problem as the dust motes are significant in size to the device dimensions and a slight layer of skin oil can negate the effectiveness of adhesives used in bonding components. Furthermore, components must be manipulated using either mechanical or vacuum tweezers which often leads to lost or destroyed parts due to mishandling.

Additionally, the use of commercially available PET tubing as part of the manufacturing process limits the control of geometries and features of the insertion tool design. For instance, the primary reason for the stacked chamber configuration for the insertion tool design is because of limitations imposed by use of the PET tubing as part of the fabrication process. Ideally, the chambers would be designed in series to reduce the insertion profile and so that each chamber would individually provide curvature control for a respective length of the implant. For these reasons, a new fabrication process was developed to improve upon future surgical implant tool performance and open up the latitude of design options. The following section outlines the new fabrication process and results.

\subsection{Lithographic Process for High Aspect Ratio Flexible Fluidic Cavities}

To overcome these obstacles, a new fabrication method has been developed, in which the chambers are manufactured using lithographic methods commonly used in the semiconductor industry and for MEMS applications. The insertion tool would be made in layers of thin polymer films spun onto a substrate in liquid form and patterned into structures using photolithographic masks and UV light. Once 
the basic tubular structures for the actuation chambers have been formed on the flat substrate, they are released and wound onto mandrels. The devices can then be baked to cure the polyimide and form the device into a three-dimensional structure.

The basic flow for the process to build up and pattern polymer films into actuation chambers is outlined in Figure 5.1. The first step in the process bonds the silicon electrode arrays onto a release layer in the substrate. This simplifies the integration between the insertion tool and probe by negating the need for an additional bonding step requiring adhesives that are difficult to apply. The next layer consists of a polyimide post and sacrificial layer of poly-methyl-methacrylate (PMMA). The post will act as the bonding link between the backing chambers and the silicon electrode array and allow the chambers leeway in changing their cross section geometry during pressurization. The sacrificial layer of PMMA acts as a base onto which the chambers will be built.

Step 4 of the process is to lay down the base of the chambers in polyimide $(4 \mu \mathrm{m}$ thick). Next, a sacrificial layer of PMMA is laid down to act as core material for fluid ducts that will run from the pressure source to the chambers. A $2 \mu \mathrm{m}$ layer of polyimide is then used as the structure for these ducts. In Step 7, several layers of PMMA are layered and patterned to achieve a thickness of $15-25 \mu \mathrm{m}$ that create the chamber cores.

The walls of the core will have a sloped profile to facilitate the penetration of UV light into the polyimide layer that creates the structure of the chambers. Instead of building the chamber walls and top in several layers, the surface will be flood-coated in polyimide and spun to produce a layer $4 \mu \mathrm{m}$ thick on both the top surface and the sidewalls of the PMMA core.

The final step of the lithography process is to release the structures from 
1. Embed silicon probe in release layer

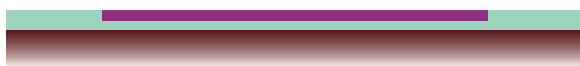

3. Base of chamber in polyimide

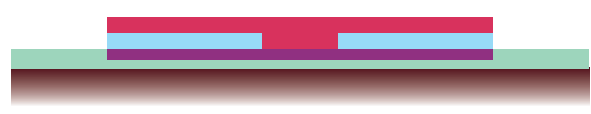

5. Polyimide layer for fluid duct structures

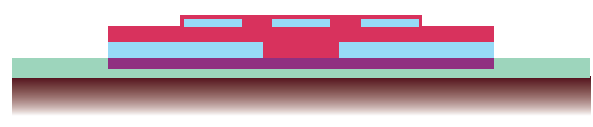

7. Polyimide layer to form chamber walls

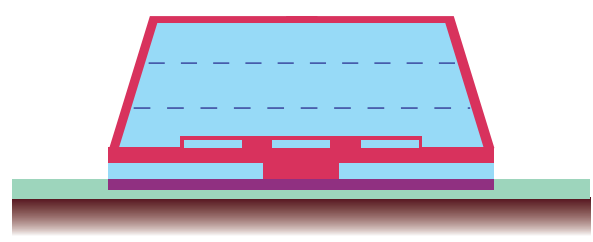

Substrate

Release Layer

Silicon Probe
2. Polyimide post and PMMA sacrificial layer



4. PMMA cores for fluid ducts

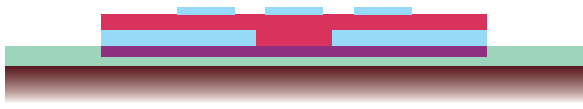

6. Multi-layer PMMA core for chambers

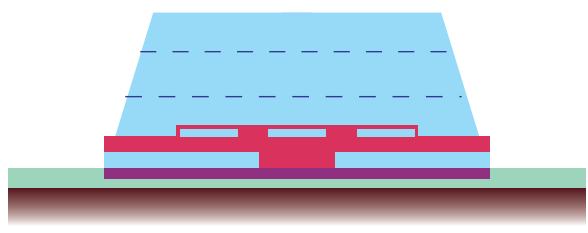

8. Release device by disolving release layer



PMMA



- - - Layer Boundary

Figure 5.1 Lithographic process steps for a polyimide insertion tool. 
the substrate surface. It is important to note that while the polyimide films have been baked to drive off solvents and form solid layers, the polyimide has not been polymerized. Polymerization is performed at a higher temperature when the released structures are wound onto a mandrel to induce the final helical shape for the device. After winding, the device is baked at $300^{\circ} \mathrm{C}$ to simultaneously polymerize the polyimide and lock it into the wound helical configuration and to pyrolyze (vaporize) the PMMA sacrificial layers opening up the chambers and ducts.

A conceptual drawing of the insertion device fabricated with such a process is shown in Figure 5.2. The new design has an in-line chamber configuration with the actuation chambers aligned end to end in discrete portions rather than the stacked configuration. This will reduce the overall size of the device allowing more room for movement within the cochlea. Also, each longitudinal section of the tool can be controlled individually rather than cumulatively as in the stacked design.

With lithography the dimensions and shape of the backing are more tightly controlled allowing greater latitude in the design and greater predictability in performance. In the conceptual drawing, the profile of the backing tool is shown to exactly match the tapering profile of the silicon electrode array. However, the width can be specified anywhere along the length of the tool to adjust actuation ability as needed, something impossible with the PET tubing which only allow for two widths.

Another, improvement over the old design is the interface with the pressure source. The PET chambers required a steel hypo interface which is difficult to make connections with. The polyimide backing tool will have open ports on the top surface leading to the fluid ducts. To this array of ports can be mounted an set of miniature electrokinetic pumps built into one body such as the three pump 


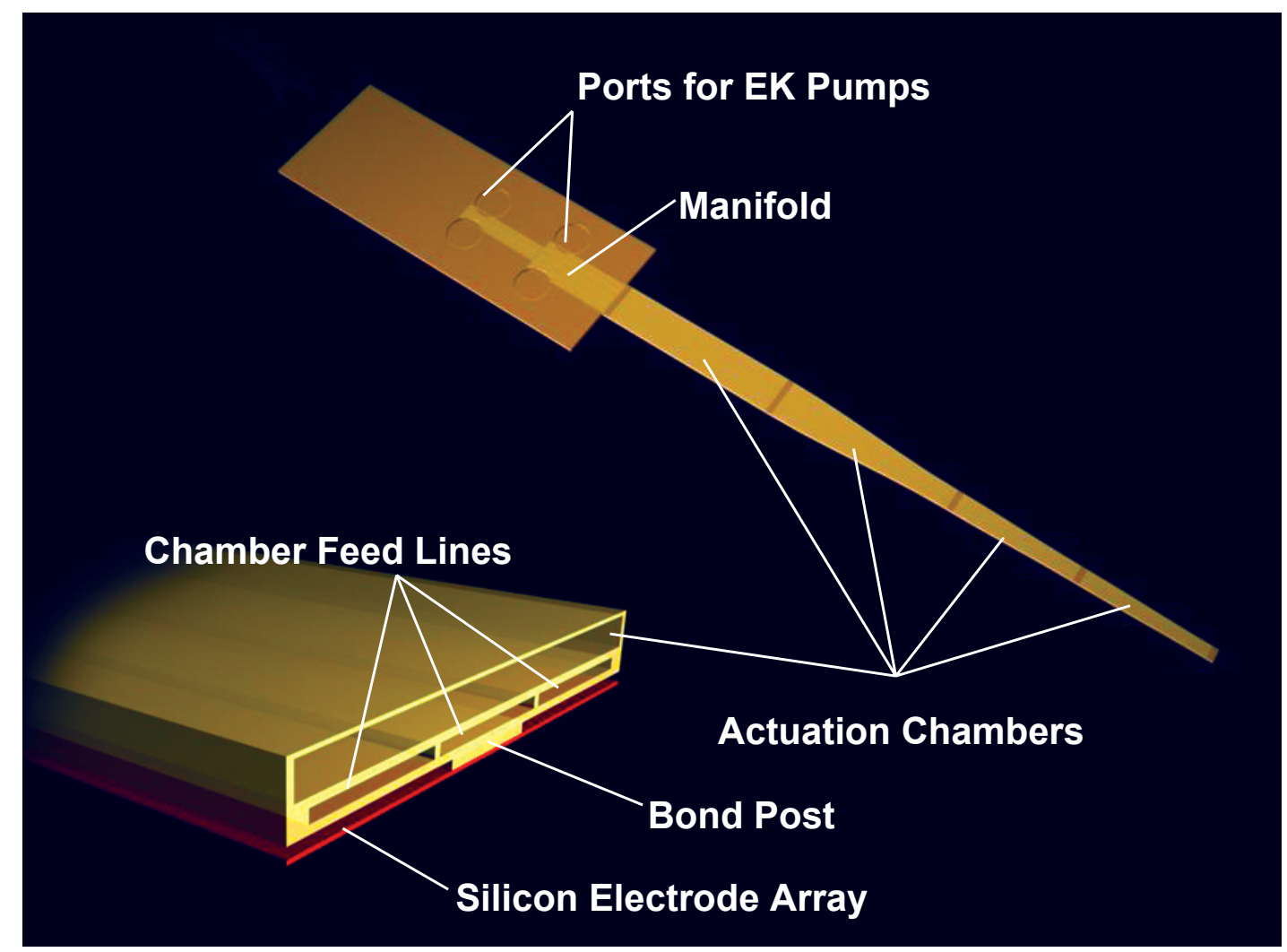

Figure 5.2 Conceptual diagram for an insertion tool fabricated using the lithography polyimide process.
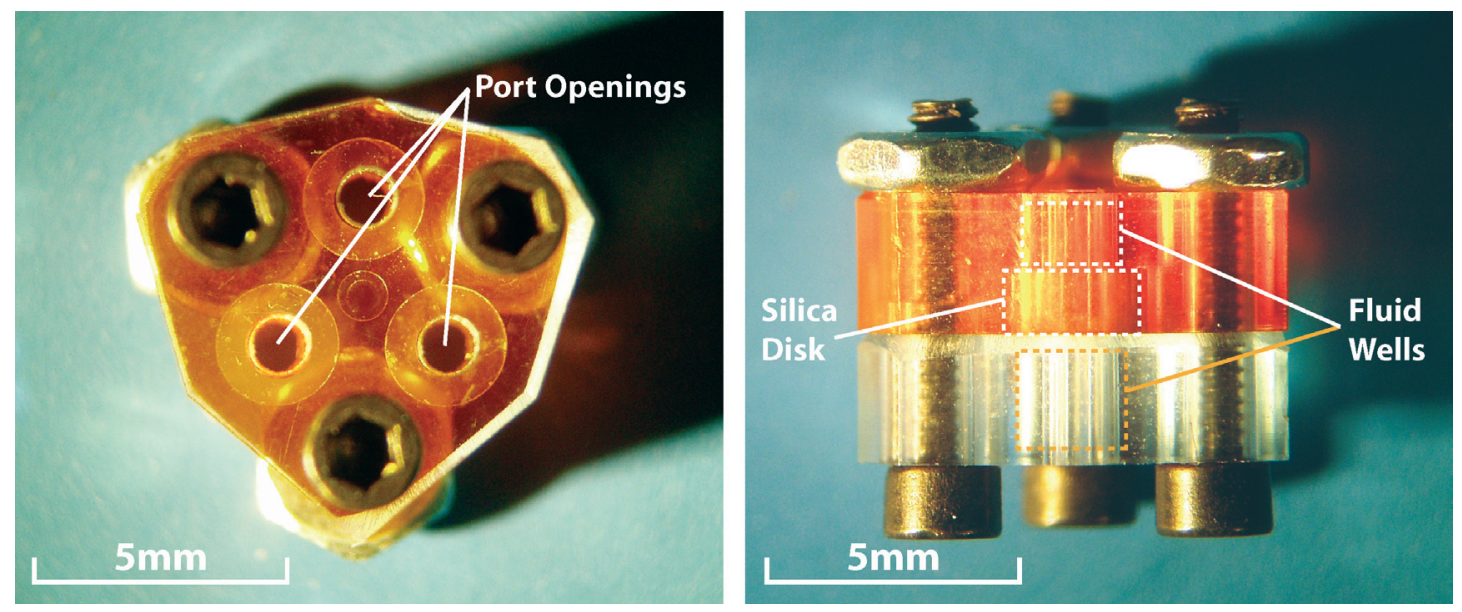

Figure 5.3 A minuterized three EK pump bank. 
design that is currently under development by Dr. Craig Friedrich (Fig. 5.3).

\subsection{Results of Fabrication for the Lithographic Process}

The following section details the initial fabrication results using the lithographic process. The process was performed in the clean room facilities located at Michigan Technological University and focused on developing the lithographic process rather than on building advanced insertion tools.

The substrates used in the process were $100 \mathrm{~mm}$ diameter silicon wafers with a sputtered coating of chrome on the surface. The chrome acts as a sacrificial layer that will later be dissolved from beneath the fabricated devices to release them using a chrome or other etchant.

The first step in the process was to deposit a layer of polyimide for the base of the device using a spinner. The silicon substrate is vacuum chucked to the spin head and a pool of photo definable polyimide is pooled in the center of the wafer using a disposable plastic pipette. The polyimide used in the lithographic devices is manufactured by HD Microsystems (PI 2723). The polyimide was chosen so as to have a good film strength and a film thickness range of around $4 \mu \mathrm{m}$ at typical spinner speeds. The polyimide was spun on at 2000 RPM for 30 seconds with the aim of achieving a $4 \mu \mathrm{m}$ final film thickness. The film was then soft baked on a hotplate for $120 \mathrm{sec}$ at $120^{\circ} \mathrm{C}$.

Patterning of the polyimide film was performed on an Electronics Visions Group (EVG) optical aligner (model EV 620) with soft contact between the mask and the soft-baked polyimide film. Exposure was continuous for 120 seconds. Soda lime masks with a chrome pattern were used for the base and top layers of polyimide in the device with the patterns for the top and bottom polyimide layers shown in Figure 5.4. 
The patterns are in negative tone resist and are repeated in 4 rows by 20 columns on the mask surface. The two patterns are identical except that the top pattern has a port opening in the surface for fluidic connections. Each pattern also features a $1000 \mu \mathrm{m} \mathrm{x}$ $1000 \mu \mathrm{m}$ square "handle" at each end with a $500 \mu \mathrm{m}$ hole in its center to aid in handling and winding about a mandrel for the curing/shaping process. Each handle is attached to the device by a $2000 \mu \mathrm{m} \times 125 \mu \mathrm{m}$ "leash". The patterns also contain a $500 \mu \mathrm{m} \mathrm{x}$ $500 \mu \mathrm{m}$ cross hair target at the distal end to aid in alignment of subsequent layers.

After exposure, the films are developed in DE1000 (HD Microsystems) for approximately 30 seconds then rinsed in deionized (DI) water. The development of the pattern at this point was checked beneath a microscope and any additional required time in the developer solution was determined.

The next step involved spinning and patterning the PMMA core layer for the device. The PMMA film used was Microchem 950PMMAA resist. The film was spun similarly to the polyimide with spin parameter settings of 1000RPM for 45 seconds with the goal of achieving a $5 \mu \mathrm{m}$ thick PMMA film. The film was soft baked at $180^{\circ} \mathrm{C}$ for 90 seconds.

The substrate was again placed in the aligner and exposed with the core mask for the PMMA layer (see Fig. 5.5). The PMMA exposure differs from the polyimide exposure because the PMMA film is only sensitive to deep UV in the 250nm range. Soda lime glass is opaque to UV light at this wavelength, and therefore the mask was made using quartz glass with a chrome pattern. The UV light from the EVG aligner is also not sufficient at this wavelength, therefore a lamp was fabricated using 6 partially overlapping 9-watt Phillips fluorescent germicidal bulbs (PL-S9W) that emit in the deep UV spectrum (Figure 5.6). 


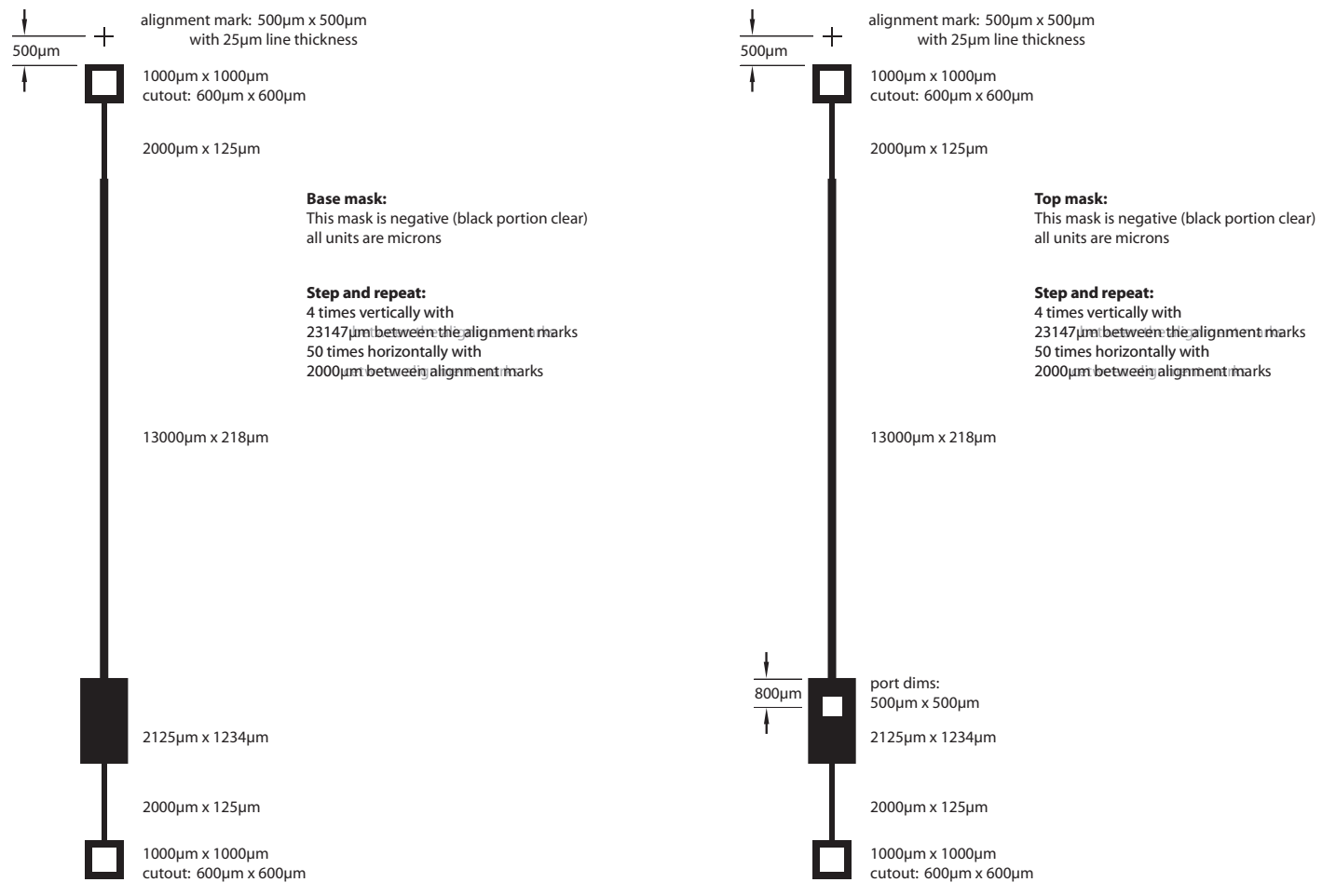

Figure 5.4 Base and top patterns for the polyimide films. All units are reported in micrometers.



Figure 5.5 Quartz core mask pattern for the PMMA layer. 




Figure 5.6 The deep UV lamp housing and ballast bank.

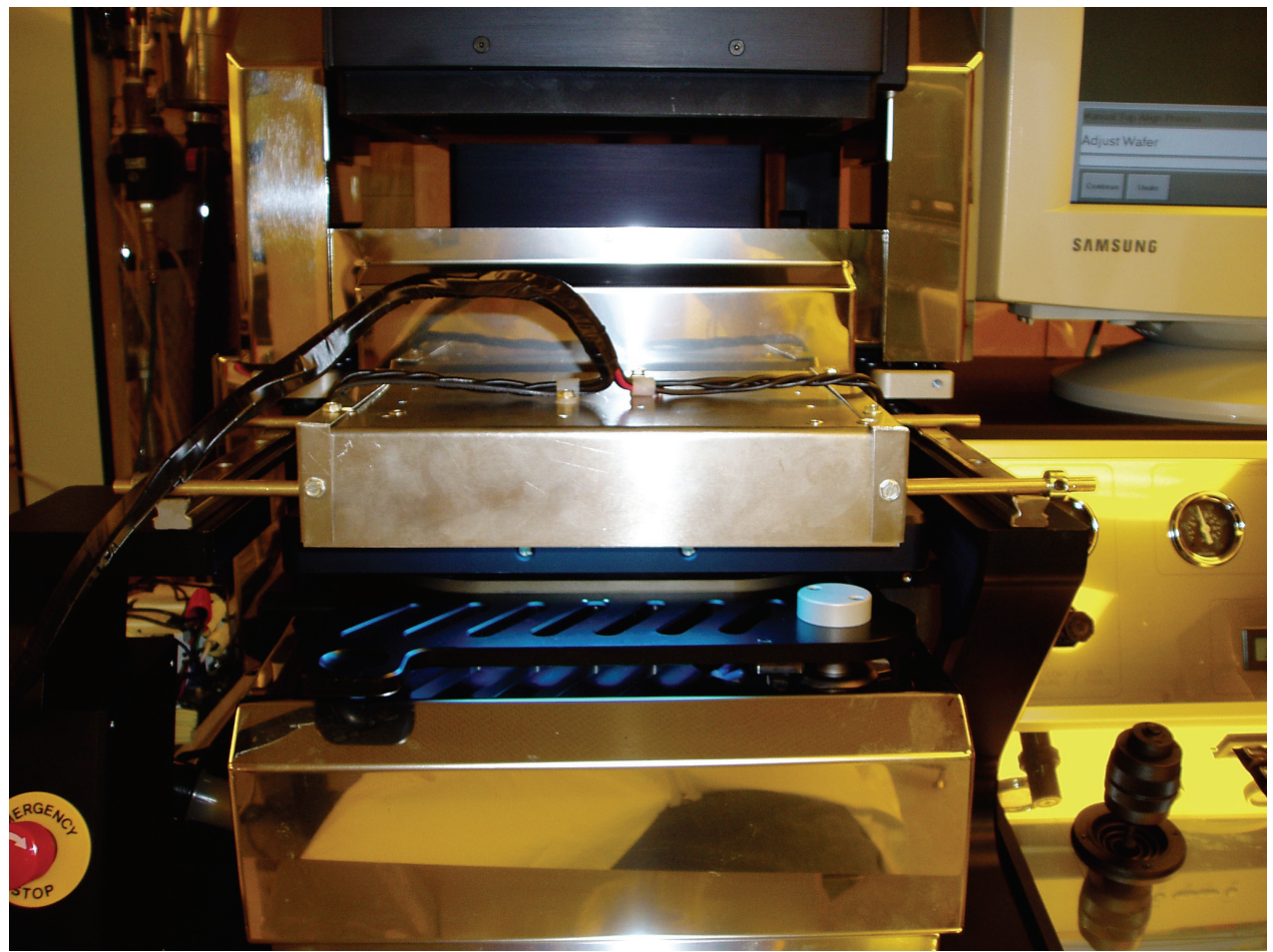

Figure 5.7 Deep UV lamp housing mounted and operating on the EVG aligner. 
The procedure for the PMMA film follows that of the polyimide base pattern underlying the PMMA film on the substrate. The core mask is aligned with the polyimde patterned film and put into soft contact. The optics for the aligner are then moved back to reveal the upper surface of the glass mask plate. The lamp is then positioned over the mask and substrate on the aligner and turned on to perform the exposure (see Figure 5.7).

Exposure of the PMMA layer with the deep UV lamp took approximately 3.5-4 hrs to complete. The film was then developed in a 1:2 MIBK:IPA (methylisobutyl-ketone:isopropyl alcohol) solution bath (MicroChem) for 3 minutes and rinsed in DI water and checked under the microscope for development of the film.

The top polyimide layer is then added using the procedure previously outlined for the polyimide with the top mask. After developing the polyimide, a basic single chamber polyimide device was fabricated. Figure 5.8 illustrates several areas of the fabricated device under a microscope. The outcome from the development of the single actuation device from the lithographic process yielded very positive results. The PMMA core is clearly evident as a bulge between the two interleaved layers of polyimide. In the tip region photograph (center), the top polyimide layer has fully formed around the narrower PMMA core and bonded to the polyimide layer below it.

At this stage, the device is completely formed on the substrate surface and only requires to be removed from the substrate and cured. Profile measurements on a white light interferometric microscope indicate that a thickness of approximately $3.5 \mu \mathrm{m}$ was achieved for the polyimide layers and $4 \mu \mathrm{m}$ for the PMMA in the port region of the device. The PMMA thickness was slightly less at about $3.5 \mu \mathrm{m}$ in the tip region. The variation in film thickness is most likely due to the spinning of the liquid polymer 



Figure 5.8 Images of a fabricated polymide device with PMMA core on the substrate surface. From top to bottom: back end with port opening to polyimide core, chamber tip attached to polyimide leash, and the handle with adjacent target. 



Figure 5.9 Interfermetric measurements of the port and tip areas for a polyimide device.

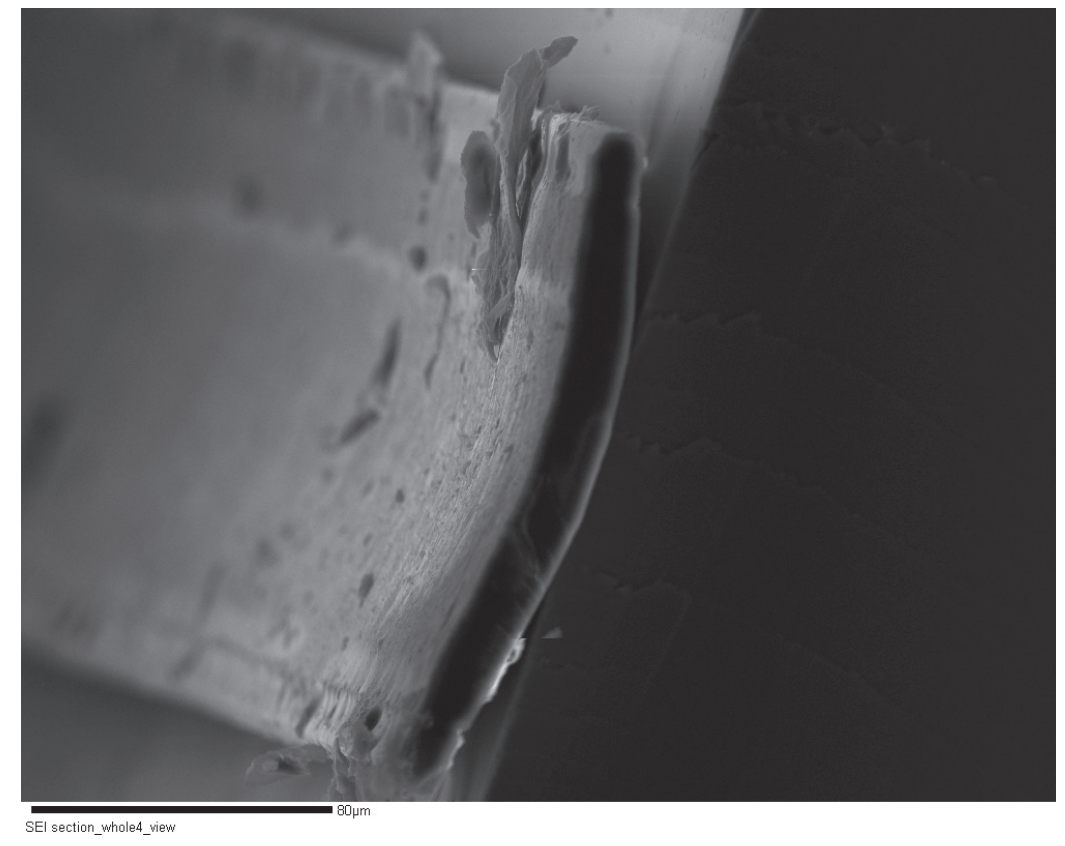

Figure 5.10 SEM image of a cured polyimide device chamber that has been sliced open to view the interior. 
since centrifugal forces vary across the wafer surface. Figure 5.9 illustrates two scans taken on the interferometric microscope for the port and tip regions of the device.

To remove the devices from the substrate, the wafer was placed into a chrome etch bath to dissolve the underlying sputtered chrome layer lying between the device and the silicon substrate. The etch took approximately $24 \mathrm{hrs}$ to complete, during which the devices began to curl back from the substrate due to minor film tensions.

The final step in completing the device involves constraining its shape on a mandrel and then baking it at $300^{\circ} \mathrm{C}$ for $3 \mathrm{hrs}$ to simultaneously cure the polyimide and evaporate the PMMA core creating a 3D-shaped chamber. The first devices were cured without shaping to observe how the PMMA layer pyrolyzed out of the polyimide layers. These devices were placed on a hotplate while the temperature was ramped to about $250^{\circ} \mathrm{C}$ over the course of $15 \mathrm{~min}$. and held there for $1 \mathrm{hr}$. Visual observation showed no break points or obvious bulges in the polyimide layers. SEM images of the chamber sliced across its length indicate that the PMMA core fully pyrolyzed leaving an open polyimide cavity (Figure 5.10).

After confirming that the device core did indeed open up during the curing process the next logical step is to attempt shaping and curing a device and attaching a pressure source to the port for the purposes of actuation. A brass manifold was machined from $150 \mu \mathrm{m}$ stock with a pocket to fit over the port opening of the device at its back end and a channel leading from the port to which a steel hypo could be inserted to provide actuating pressuring from a pneumatic source. In Figure 5.11, a polyimide device is attached to the brass manifold using liquid polyimide (nonphoto definable) with the port opening of the device facing into the brass pocket. 

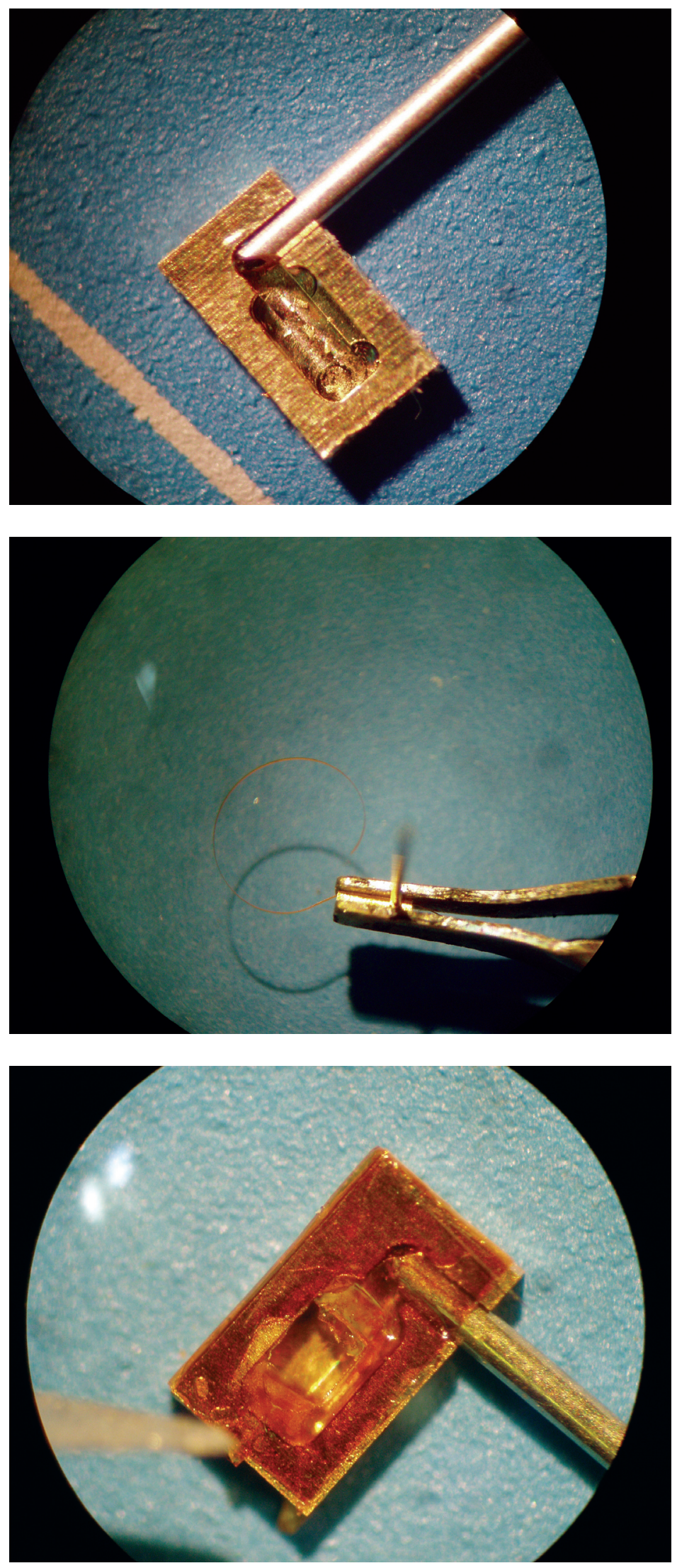

Figure 5.11 Brass manifold and steel hypo used to connect fluidic pressure to the polyimide device's port.

Figure 5.12 Cured and shaped polyimide device.

Figure 5.13 Close up of the bonded brass manifold and device port. 
While the adhesive is wet, a copper micro clip is used to clamp the brass manifold and the device together. The tip handle can then be glued to a metal winding mandrel using white glue in a process similar to that which is used to fabricate the PET chambers. The polyimide device is wound around the mandrel using the micro clip as a weight to put tension on the chamber and hold it against the mandrel face. The device was then baked to cure and form the device. The baking took place on a hot plate where the fixturing and device are placed on the surface and an inverted Pyrex beaker covers the assembly to hold in heat. A thermocouple is clamped to the winding mandrel near the device to monitor temperature. The baking temperature was slowly ramped by incrementing the temperature upwards by $50^{\circ} \mathrm{C}$ every 10 minutes and then holding at $300^{\circ} \mathrm{C}$ for a total bake time of $3 \mathrm{hrs}$.

A device in its cured and shaped form is illustrated in Figure 5.12. As can be seen from the photograph, curing forms the polyimide nicely to the constrained shape. Figure 5.13 shows a close up of the bond between the brass manifold and the polyimide device after the curing process.

To date, a successful actuation with a polyimide device has yet to be accomplished. Several attempts have been performed where the devices have been attached to brass manifolds and a pressure source (house air) without discernible actuation. Since the design and process are in the preliminary stages, there are a number of possible factors to investigate.

One likely negative influence is the affect of the chrome etchant on the polyimide film material properties. Observations made during the curing process noted that the polyimide film was noticeably lighter in color and seemed to lack the usual strength. Fracture of the device where the chamber 
meets the back end was a common occurrence during the curing process.

The effects of the etchant on the film were confirmed by releasing several devices from the chrome layer by propagating a crack through silicon substrate. A sharp pointed tool was used to notch the substrate edge repeatedly until a straight line crack formed through the crystalline silicon and under the device structures. The devices were then gently peeled from the substrate to release them. Experiments performed with these devices released without etchant were qualitatively observed to be much stronger and no breakages occurred at the chamber to back end interface point as did before during the curing procedure.

Another factor that likely precluded successful actuation was that the brass manifold lacks a channel leading from the open pocket area to the edge to allow space for the chamber thickness. During the curing step, the copper clip provides pressure to hold the brass manifold and polyimide device in contact for bonding. Since no allowance is made for the $10-15 \mu \mathrm{m}$ thick chamber leaving the manifold, the chamber is likely being sealed shut as the PMMA core is pyrolyzed.

Despite lacking evidence of a successful actuation for the polyimide device, the sum of observations and measurements indicate that the lithographic process is not only a viable alternative manufacturing method, but also potentially far more desirable to the methods for fabricating PET devices. The improved geometry control in the process makes possible many design features and dimensions that are impossible to replicate with the PET tubing methods. Furthermore, since the process entails similar techniques to that of the thin film electrode array, it is feasible that the probe and backing may one day be manufactured as a single cohesive unit rather than components requiring assembly. 


\subsection{Conclusions}

The goal of this research was to develop an articulated insertion tool to aid in the precise, low-impact and perimodiolor placement of an electrode array within the cochlea. Along the modiolar wall is the optimal placement for the effectiveness of localized stimulation of the electrodes with minimum power requirements. The insertion tool also looks to minimize contact force with the delicate structures of the cochlea during the surgical procedure to avoid intracochlear damage that might lead to degeneration of the auditory nerves. Minimizing contact force also has the added benefit of reducing friction between tool and cochlea allowing for a deeper placement of the array to reach auditory nerves responsible for a broader frequency range of auditory perception.

To achieve that goal, micro-fluidic actuation chambers were developed to adjust the curvature of a perimodiolar shaped cochlear implant device consisting of the chambers bonded to a high-density silicon electrode array. A manufacturing process for the chambers was created to fabricate prototypes of the insertion device and integrate them with silicon electrode array.

Many prototype actuation tools were fabricated to explore several avenues of design for the device. Tests of these prototypes gained a knowledge of the Bourdon type actuation principle. In-vitro insertion experiments proved the validity of the device for cochlea implantation procedures of highly flexible electrode arrays.

Computer modeling garnered insight into the mechanics of Bourdon type actuation and will benefit future design efforts for these types of highly flexible actuators. Furthermore, the computer models have laid the ground work for developing the current technology into a fully-automated articulated cochlea implant system. 
Finally, a next generation thin film lithography process has been developed to improve the performance, dimensions and range of design options for future actuation tools. Using this method, prototype actuation tools have been fabricated from thin films of polyimide and PMMA into three-dimensional, high-aspect ratio chambers with refined geometry that can accommodate multiple wafer level EK pumps.

\subsection{Recommendations for Future Research}

The vision for the ultimate design goal would be to combine the manufacturing technologies, modeling from actuation principles, the improved actuating source of EK pumps and probe level sensor information from strain and touch sensors into a cohesive cochlear implantation system. In order to achieve that end, the following areas of research would prove beneficial:

- Improve upon the lithographic process by adding a sacrificial release layer that does not harm the polyimide/PMMA device when dissolved. A candidate technology might be electrolysis of an aluminum sacrificial layer with an underlying gold conductive layer [38].

- Further refinement of EK pump technology to a wafer level design where several pumps built into an array could be bonded directly to the actuation chambers to provide fluidic pressure.

- A control system that utilizes strain and touch sensor information on the probe level sensors could accurately and automatically control the actuation of an implant.

- Apply the actuation technology beyond cochlear implants. One promising area might be in adding steering potential for deep brain probes.

- The problem of chamber lock-up is a puzzling phenomenon that merits further investigation. 


\section{References}

1 Grey Henry. Gray's Anatomy, 15th Edition, Bounty Books, New York, 1977, 859-68.

2 "Ear Anatomy" Atlantic Coast Ear Specialists, Virginia Beach, Virginia, website http://www.earaces.com/anatomy.htm

3 American Speech-Language-Hearing Assosiaction. (2004). Technical Report: Cochlear Implants. ASHA Supplement 24. in press.

4 Kennedy DW. Multichannel intracochlear electrodes: mechanism of insertion trauma. Laryngoscope 1987;97:42-49.

5 Niparko JK, Kemink JL, Oviatt DL, Altschuler RA. Evaluation of the temporal bones of multichannel cochlear implant patient. Ann Otol Laryngol 1991;100:914-21.

6 Chen JM, Farb F, Hanusaik L, Shipp D, Nedzelski JM. Depth and quality of electrode insertion. The American Journal of Otology 1999;20:192-7.

7 Blamey PJ, Pyman BC, Dowll RC, et al. Factors predicting post-operative sentance scores in postlingually deaf cochlear implant patients. Ann Otol Rhinol Laryngol 1992;101:342-8.

8 Cords SM, Reuter G, Issing PR, Sommer A, et al. A silastic positioner for modiolus-hugging position of intracochlear electrodes: electrophysiologic effects. The American Journal of Otology 2000;21:212-217.

9 Frijns JHM, Briaire JJ, Grote JJ. The importance of human cochlear anatomy for the results of modiolus-hugging multichannel cochlear implants. Otology \& Neurotology 2001;22:340-349.

10 Kawano A, Sheldon HL, Clark GM, Ramsden RT, Raine CH. Intracochlear factors contributing to psyschophysical percepts following cochlear implantation. Acta Otolaryngol (Stock) 1998;118:313-26.

11 Shepard RK, Hatsushika S, Clark GM. Electrical Stimulation of the auditory nerve: the effect of electrode position on neural excitation. Hear Res 1993;66:108-22.

12 Brummer SB, Turner MJ. Electrochemical considerations for safe electrical stimulation of the nervous system with platinum electrodes. IEEE Trans Biomed Eng 1977;24:59-62.

13 Roland JT Jr., Fishman AJ, Alexiades G, Cohen NL. Electrode to modiolus proximity: a fluoroscopic and histologic analysis. The American Journal of Otology 2000;21:218-225. 
14 Kumakawa K, Takeda H, Ujita N. Determining the optimum insertion length of electrodes in the cochlear 22-channel implant: Results of a clinical study. Adv Otorhinolaryngol 1997;52:129-34.

15 Kennedy DW. Multichannel intracochlear electrodes: mechanism of insertion trauma. Laryngoscope 1987;97:42-49.

16 "Cochlear" Nucleus 24 Contour, Cochlear Corporation, website http://www.cochlear.com/912.asp

17 "Electrode history," CLARION System, Advanced Bionics, website http://www. cochlearimplant.com/shortcut_hifocus_edh.html

18 "The design and development of a peri-modiolar electrode array," Nucleus 24 Contour, website http://www.ci-centrum.de/CI-Info/Nucleus\%204pp.pdf

19 "Cochlear Implant Recipients May be at a Greater Risk for Meningitus" SEE/ HEAR Newsletter, website http:/www.tsbvi.edu/Outreach/seehear/winter03/ fda.htm

20 "Have you Heard?" Iowa Department of Public Health Newsletter 2003;10:3,5 website http://www.idph.state.ia.us/iaehdi/Newsletter/Feb03.pdf

21 “MRM Home Page" Department of Cell Biology an Anatomy, University of North Carolina at Chapel Hill, website http://www-cellbio.med.unc.edu/ henson_mrm/

22 "Ear" The University of Kansas Medical Center, website http://www.kumc.edu/ instruction/medicine/anatomy/histoweb/eye_ear/ear02.htm

23 Voie AH, Spelman FA. Three Dimensional Reconstruction and Quantitative Analysis of the Mammalian Cochlea. Doctoral Dissertation, University of Washington, 1996.

24 Pfingst Brian E, direct correspondence, Kresge Hearing Reasearch Institute, University of Michigan, Oct. 2002.

25 Lee Kunwoo. Principles of $C A D / C A M / C A E$ Systems. Reading, MA: AddisonWesley, 1999.

26 Bhatti PT, Arcand BY, Wang J, Butala NV, Friedrich CR, and Wise KD, “A High-Density Electrode Array for a Cochlear Prosthesis," IEEE International Conference on Solid-State Sensors and Actuators (Transducers'03), Boston, pp. 1750-3, June 2003.

27 Wang J, Gulari M, Bhatti PT, Arcand BY, Friedrich CR and Wise KD, "A Cochlear Electrode Array with Built-in Position Sensing", 18th IEEE International Conference on Micro Electro Mechanical Systems, Miami, Jan 30 - Feb 3, 2005 
28 Wolf, Alfred, "An Elementary Theory of the Bourdon Gage", Journal of Applied Mechanics, pp A207-10, Sep. 1946.

29 Conway, Cynthia D., Analytical Analysis of Tip Travel in a Bourdon Tube. Masters Thesis, Naval Postgraduate School, Monterey, CA, Dec. 1995.

30 Butala NV, An Actuated Cochlear Prosthesis Insertion Tool. Masters Thesis, Michigan Technological University, Houghton, MI, 2003.

31 “Advanced Polymers Inc.” Advanced Polymers Inc., website http://www. advpoly.com/

32 Shyamsunder S, An Electrokinetic Pumping System for a Cochlear Implant Insertion Tool, Masters Thesis, Michigan Technological University, Houghton, MI, 2004.

33 "Yager Group Home Page, The University of Washington", website http://faculty.washington.edu/yagerp/microfluidicstutorial/basicconcepts/ basicconcepts.htm

34 Zeng S, Chen CH, Mikkelsen JC Jr., Santiago JG, "Fabrication and Characterization of Electroosmotic Micropumps", Sensors and Actuators, B 79 (2001) 107-114, 2001.

35 Roark R, Young W. Formulas for Stress and Strain, 5th Edition, Mc Graw Hill, New York. 248-8, 1975. 


\section{Appendix A: $\quad$ Cochlear Geometry and Programs \& Modeling}

\section{MATLAB program code $g p \_$geometry. $m$}

This program performs calculations on the data sets st_data.mat and rc_data.mat which contain $3 \mathrm{D}$ points for cross sections defining a guinea pig scala tympani and Rosenthal's canal respectively.

$\%$ Voie-Spelman data manipulation and equation fitting

$\%$ st $=$ data on guinea pig scala tympani cross sections

$\%$ st_last=index numbers corresponding to last data point for each st cross section

$\%$ st_centers $=3 \mathrm{~d}$ coordinate of cross section center

$\%$ rc, rc_last, rc_centers $=$ Rosenthal's Canal data

$\%$

\section{$\%$ Settings}

pon $=0 ; \quad \%$ plotting on/off

\%Load Voie-Spelman Data

disp('Loading Voie-Spelman data...')

load st_data.mat

load rc_data.mat

\%swap data order to match coordinates system $[\mathrm{x}, \mathrm{y}, \mathrm{z}]$ and translate to center the base on $[0,0,0]$

disp('transform data...')

trans $=[1-1.25-2]$;

st $2(:, 1)=\operatorname{st}(:, 3)+\operatorname{trans}(1)$;

$\operatorname{st} 2(:, 2)=\operatorname{st}(:, 1)+\operatorname{trans}(2)$;

$\operatorname{st} 2(:, 3)=\operatorname{st}(:, 2)+\operatorname{trans}(3)$;

$\operatorname{stc} 2(:, 1)=$ st_centers $(:, 3)+\operatorname{trans}(1)$;

$\operatorname{stc} 2(:, 2)=$ st_centers $(:, 1)+\operatorname{trans}(2)$;

$\operatorname{stc} 2(:, 3)=$ st_centers $(:, 2)+\operatorname{trans}(3)$;

\%Remove repeated point from each st cross section

first $=1$;

first_new $=1$;

for $\overline{\mathrm{ii}}=1$ :length(st_last)

last $=$ st_last(ii);

st_last_new(ii)=st_last(ii)-ii;

st 2 _new(first_new:st_last_new(ii),:)=st2(first:last-1,:);

first $=$ last +1 ;

first_new=st_last_new(ii)+1;

end 
clear st2

clear st_last

st2=st2_new;

st_last $=$ st_last_new;

$\operatorname{rc} 2(:, 1)=\operatorname{rc}(:, 3)+\operatorname{trans}(1)$;

$\operatorname{rc} 2(:, 2)=\operatorname{rc}(:, 1)+\operatorname{trans}(2)$;

$\operatorname{rc} 2(:, 3)=\operatorname{rc}(:, 2)+\operatorname{trans}(3)$;

$\operatorname{rcc} 2(:, 1)=$ rc_centers $(:, 3)+\operatorname{trans}(1)$;

$\operatorname{rcc} 2(:, 2)=$ rc_centers $(:, 1)+\operatorname{trans}(2)$;

$\operatorname{rcc} 2(:, 3)=r c \_$centers $(:, 2)+\operatorname{trans}(3)$;

$\%$ Rotates data in $\mathrm{x}$ and $\mathrm{y}$ plane

theta $=.11$;

phi=-.1;

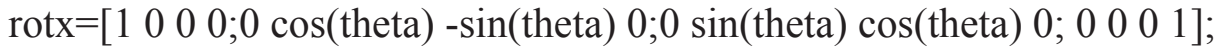

roty $=[\cos ($ phi) $0 \sin ($ phi) $0 ; 0100 ;-\sin ($ phi) $0 \cos ($ phi) $0 ; 00001]$;

$s t x=\operatorname{rotx} *[\operatorname{st} 2(:, 1)$ st2(:,2) st2(:,3) ones(length(st2),1)]';

$\operatorname{stx}=\operatorname{roty} *\left[\operatorname{stx}(1,:)^{\prime} \operatorname{stx}(2,:)^{\prime} \operatorname{stx}(3,:)^{\prime}\right.$ 'ones(length(st2),1)]';

$\operatorname{stcx}=\operatorname{rotx} *[\operatorname{stc} 2(:, 1) \operatorname{stc} 2(:, 2) \operatorname{stc} 2(:, 3) \text { ones}(\text { length}(\operatorname{stc} 2), 1)]^{\prime} ;$

$\operatorname{stcx}=\operatorname{roty} *\left[\operatorname{stcx}(1,:)^{\prime} \operatorname{stcx}(2,:)^{\prime} \operatorname{stcx}(3,:)^{\prime} \operatorname{ones}(\text { length}(\operatorname{stc} 2), 1)\right]^{\prime}$;

$\operatorname{rcx}=\operatorname{rotx} *[\operatorname{rc} 2(:, 1) \operatorname{rc} 2(:, 2) \operatorname{rc} 2(:, 3)$ ones(length(rc2),1)]';

$\operatorname{rcx}=\operatorname{roty}{ }^{*}\left[\operatorname{rcx}(1,:)^{\prime} \operatorname{rcx}(2,:)^{\prime} \operatorname{rcx}(3,)^{\prime}\right.$ ' ones(length(rc2),1)]';

$\operatorname{rccx}=\operatorname{rotx} *[\operatorname{rcc} 2(:, 1) \operatorname{rcc} 2(:, 2) \operatorname{rcc} 2(: ; 3)$ ones(length $(\operatorname{rcc} 2), 1)]^{\prime} ;$

$\operatorname{rccx}=\operatorname{roty}{ }^{*}\left[\operatorname{rccx}(1,:)^{\prime} \operatorname{rccx}(2,)^{\prime} \operatorname{rccx}(3,)^{\prime}\right.$ ' ones(length(rcc2),1)]';

disp('Fitting Scala Tympani Features...')

crop $=30 ; \quad \%$ Crop off st sections at cochleostomy, the approximate insertion area

$\%$ (proof: Brian Finsk measurements)

center $=\left[\begin{array}{ll}0.0645-0.0936\end{array}\right] ; \quad \%$ Estimated center of spiral (from visual inspection)

$\%$ Angle into cochlea

disp(' Finding Angular Depth...')

tst=zeros(length(stcx)-crop, 1);

ast=zeros(length( $\operatorname{stcx})$-crop, 1$)$;

offset_angle=atan $((\operatorname{stcx}(2, \operatorname{crop})-\operatorname{center}(2)) /(\operatorname{stcx}(1, \operatorname{crop})-\operatorname{center}(1)))$;

for $\mathrm{ii}=\operatorname{crop}+1$ :length $(\operatorname{stcx})$

$\operatorname{tst}($ ii-crop +1$)=\operatorname{atan}((\operatorname{stcx}(2, i i)-\operatorname{center}(2)) /(\operatorname{stcx}(1$, ii $)-\operatorname{center}(1)))-$ offset_angle; 





$[\mathrm{vx}, \mathrm{vy}, \mathrm{vm}, \mathrm{vang}]=$ make_in_plane(st_cs_dat,stcx $(1: 3, \mathrm{ii}))$;

[geo.st.el.majrad(cc),geo.st.el.minrad(cc),geo.st.el.tilt(cc),geo.st.el percerr(cc),geo.st.el.rmserrf(cc)]=fit_ellipse(vx,vy,vm,vang,pon);

end




$\operatorname{disp}\left({ }^{6} \quad \ldots\right.$.rmin')

[fit.st.cs.rmin.poly]=curvefit_poly(geo.st.a,geo.st.cs.rmin,3:7);

[fit.st.cs.rmin.pwr]=curvefit_power_exp(geo.st.a,geo.st.cs.rmin);

[fit.st.cs.rmin.exp]=curvefit_exp(geo.st.a,geo.st.cs.rmin,1:5);

$\operatorname{disp}($ ' $\quad .$. major radius')

[fit.st.el.majrad.poly]=curvefit_poly(geo.st.a,geo.st.el.majrad,3:7);

[fit.st.el.majrad.pwr]=curvefit_power_exp(geo.st.a,geo.st.el.majrad);

[fit.st.el.majrad.exp] =curvefit_exp(geo.st.a,geo.st.el.majrad,1:5);

$\operatorname{disp}\left({ }^{6} \quad\right.$...minor radius')

[fit.st.el.minrad.poly] $=$ curvefit_poly(geo.st.a,geo.st.el.minrad,3:7);

[fit.st.el.minrad.pwr]=curvefit_power_exp(geo.st.a,geo.st.el.minrad);

[fit.st.el.minrad.exp]=curvefit_exp(geo.st.a,geo.st.el.minrad,1:5);

$\operatorname{disp}\left({ }^{\prime} \quad\right.$...tilt')

[fit.st.el.tilt.poly]=curvefit_poly(geo.st.a,geo.st.el.tilt,3:7);

[fit.st.el.tilt.pwr] =curvefit_power_exp(geo.st.a,geo.st.el.tilt);

[fit.st.el.tilt.exp]=curvefit_exp(geo.st.a,geo.st.el.tilt,1:5);

disp('Program Completed')

\section{MATLAB program code $s$ t_reconstructor.m}

Program creates a graphical shell representation of the parametric equations defining cochlea geometry outputted by gp_geometry.m.

function $[$ out $]=$ st_reconstructor2(in)

res $=25 ; \quad \%$ Number of points per cross section

cs_num $=80 ; \quad \%$ Number of cross section in graphic

fourth=round(res/4);

$\%$ Parametric equations defining geometry

c. $\mathrm{a}=\left[\begin{array}{llllll}8.7841 \mathrm{e}-05 & -3.5867 \mathrm{e}-03 & 5.3266 \mathrm{e}-02 & -3.1455 \mathrm{e}-01 & 1.2353 \mathrm{e}-01 & 8.1752 \mathrm{e}+00\end{array}\right.$

$3.3775 \mathrm{e}+01-4.4076 \mathrm{e}-02]$;

c.r $=[2.296 \mathrm{e}-12-7.707 \mathrm{e}-9$ 8.745e-6 $-4.215 \mathrm{e}-3$ 1.351];

$\mathrm{c} . \mathrm{h}=[6.464 \mathrm{e}-13$-1.797e-9 8.012e-7 3.016e-3 -3.79e-2];

c.maj $=[9.403 \mathrm{e}-13$-3.195e-9 4.023e-6 $-2.279 \mathrm{e}-3$ 0.711];

c.min $=[8.526 \mathrm{e}-13-2.752 \mathrm{e}-93.314 \mathrm{e}-6-1.860 \mathrm{e}-3$ 0.529];

c.tilt $=[1.009 \mathrm{e}-12-2.343 \mathrm{e}-9$ 1.780e-6 $-9.491 \mathrm{e}-4.71]$;

fign=figure;

set(fign,'visible','off')

for ii $=0: 1358 /$ cs_num: 1358

major=polyval(c.maj,ii);

minor=polyval(c.min,ii);

tilt=polyval(c.tilt,ii)/180*pi; 


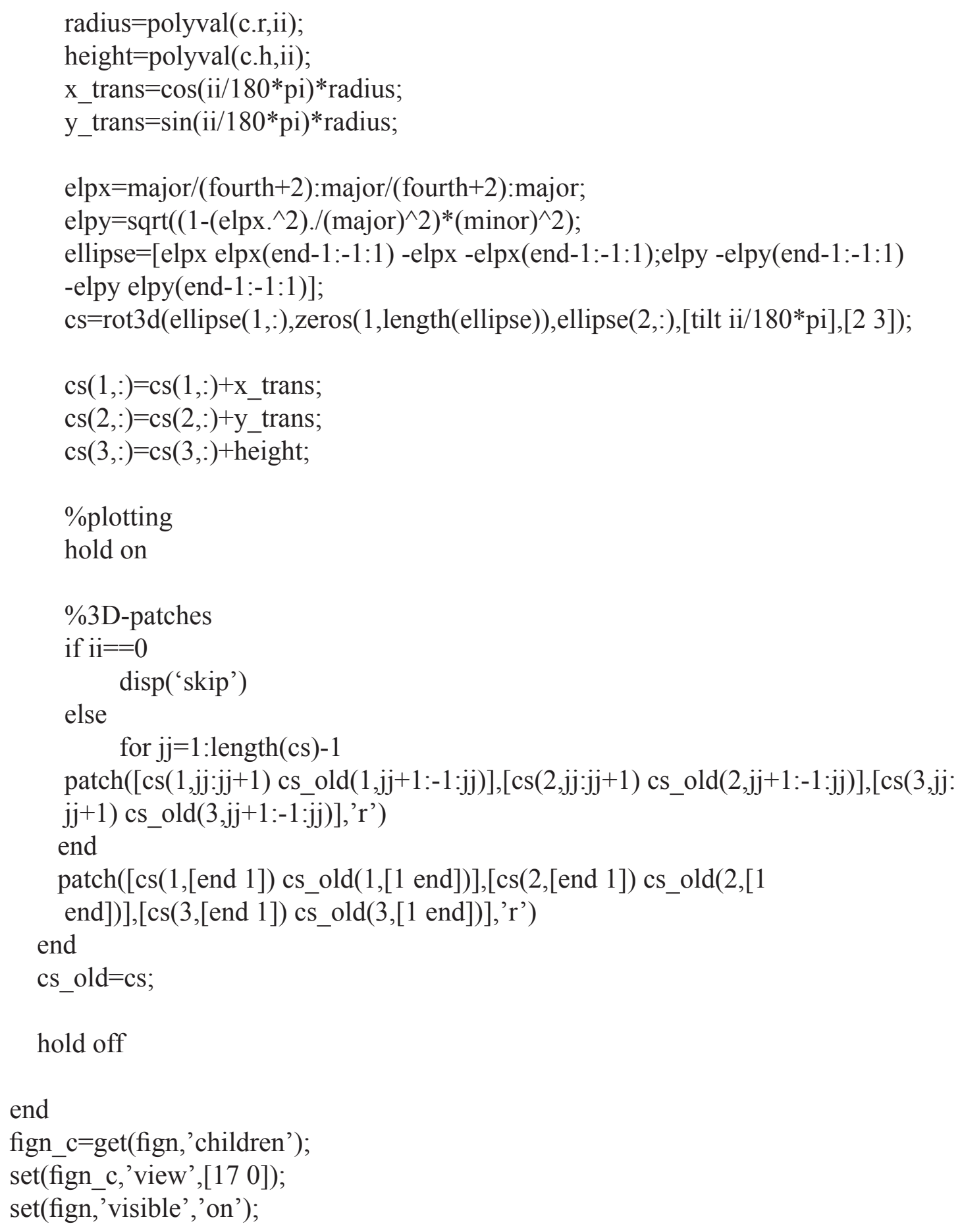




\section{Appendix B: $\quad$ Machining Programs for the Micromilling Ma- chine}

Dover milling machine code gp2dcav.pmc

Used to machine 2D guinea pig models for insertion testing of actuation chambers..

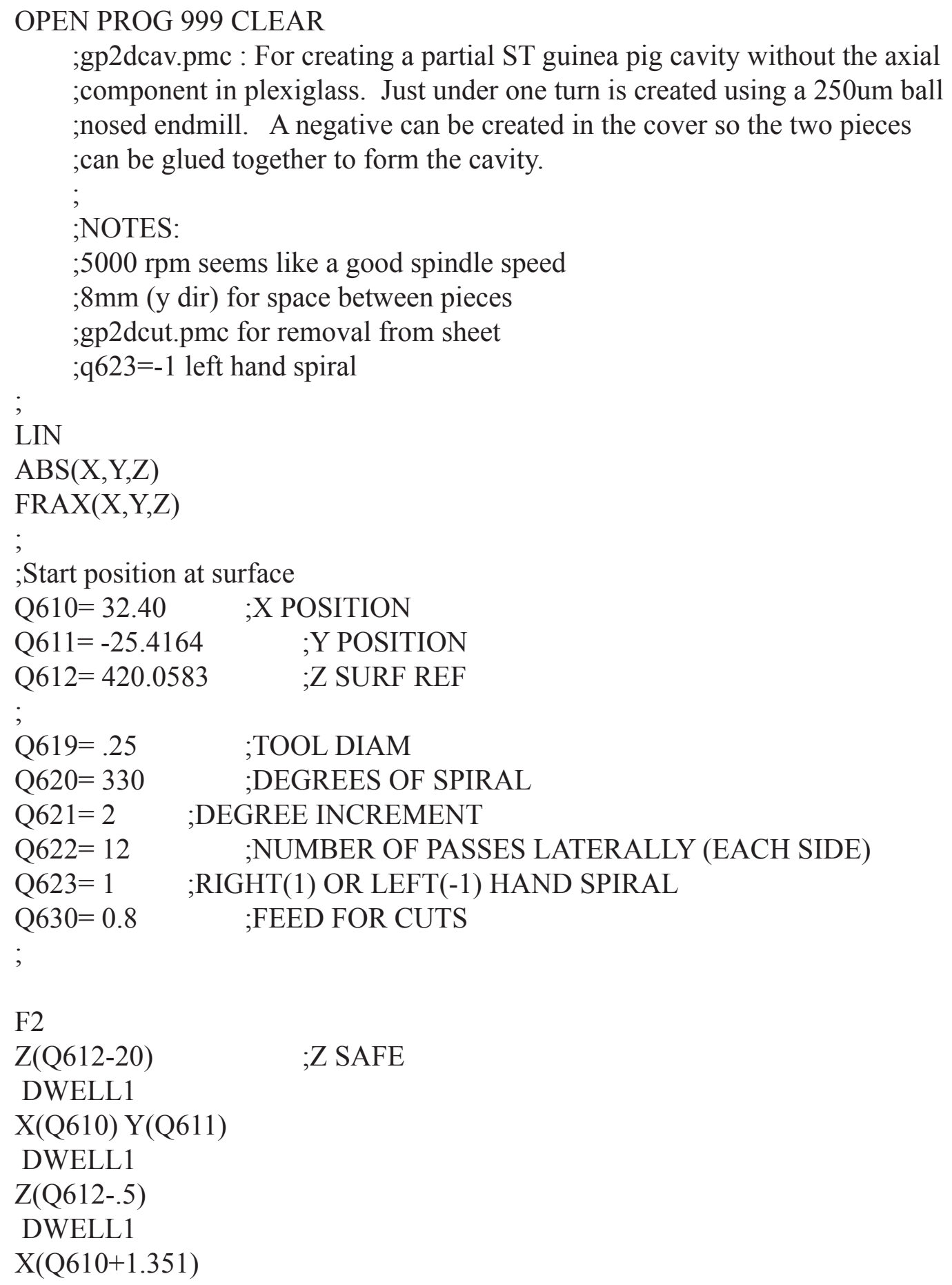


DWELL1

$\mathrm{F}(\mathrm{Q} 630)$

;

Q699 $=0$

Q690=.5 ;DO HALF DEPTH ON THE FIRST CUT

;

WHILE(Q699!>Q622)

$\mathrm{Q} 698=0$

F(Q630*.2) ;SLOW MOVE FOR START OF CUT

WHILE(Q698!>Q620)

;RADIUS

$\mathrm{Q} 640=2.296 * \operatorname{EXP}(-12 * \mathrm{LN}(10)) * \operatorname{EXP}(4 * \mathrm{LN}(\mathrm{Q} 698))-7.707 * \mathrm{EXP}(-9 * \mathrm{LN}(1$

$0)) * \operatorname{EXP}(3 * \mathrm{LN}(\mathrm{Q} 698))+8.745 * \operatorname{EXP}(-6 * \mathrm{LN}(10)) * \operatorname{EXP}(2 * \mathrm{LN}(\mathrm{Q} 698))-4.215 * \operatorname{EXP}(-$

$3 * \mathrm{LN}(10)) * \mathrm{Q} 698+1.351$

;MAJOR CS RADIUS

$\mathrm{Q} 641=9.403 * \mathrm{EXP}(-13 * \mathrm{LN}(10)) * \operatorname{EXP}(4 * \mathrm{LN}(\mathrm{Q} 698))-3.195 * \operatorname{EXP}(-9 * \mathrm{LN}(1$

$0)) * \operatorname{EXP}(3 * \mathrm{LN}(\mathrm{Q} 698))+4.023 * \operatorname{EXP}(-6 * \mathrm{LN}(10)) * \operatorname{EXP}(2 * \mathrm{LN}(\mathrm{Q} 698))-2.279 * \operatorname{EXP}(-$

$3 * \mathrm{LN}(10)) * \mathrm{Q} 698+0.711$

;MINOR CS RADIUS

$\mathrm{Q} 642=8.526 * \mathrm{EXP}(-13 * \mathrm{LN}(10)) * \operatorname{EXP}(4 * \mathrm{LN}(\mathrm{Q} 698))-2.752 * \operatorname{EXP}(-9 * \mathrm{LN}($

$10)) * \operatorname{EXP}(3 * \mathrm{LN}(\mathrm{Q} 698))+3.314 * \operatorname{EXP}(-6 * \mathrm{LN}(10)) * \operatorname{EXP}(2 * \mathrm{LN}(\mathrm{Q} 698))-1.86 * \mathrm{EXP}(-$

$3 * \mathrm{LN}(10)) * \mathrm{Q} 698+0.529$

;

;LATERAL MOVE PERCENTAGE

Q643=Q699/Q622

;MOVE DISTANCE

Q644=(Q641-Q619/2)*Q643

;DEPTH AT LATERAL POSITION

Q645=SQRT((1-((Q644*Q644)/(Q641*Q641)))*Q642*Q642)*Q690

;

;MOVE POSITIONS

;RADIUS

$\mathrm{Q} 650=\mathrm{Q} 640+\mathrm{Q} 644$

$\mathrm{Q} 651=\mathrm{COS}(\mathrm{Q} 698) * \mathrm{Q} 650 \quad ; \mathrm{X} \mathrm{MOVE}$

$\mathrm{Q} 652=\mathrm{SIN}(\mathrm{Q} 698) * \mathrm{Q} 650 \quad ; \mathrm{Y} \mathrm{MOVE}$

;

$\mathrm{X}(\mathrm{Q} 610+\mathrm{Q} 651) \mathrm{Y}(\mathrm{Q} 611+\mathrm{Q} 652 * \mathrm{Q} 623) \mathrm{Z}(\mathrm{Q} 612+\mathrm{Q} 645)$

$\mathrm{F}(\mathrm{Q} 630)$

Q698=Q698+Q621;CYCLE COUNT FOR SPIRAL PROGRESSION ENDWHILE

Q690=1 ;DO FULL DEPTH CUT FROM NOW ON

F(Q630*.2) ;SLOW MOVE FOR START OF CUT 


\section{WHILE(Q698!<0)}

;RADIUS

$\mathrm{Q} 640=2.296 * \operatorname{EXP}(-12 * \mathrm{LN}(10)) * \operatorname{EXP}(4 * \mathrm{LN}(\mathrm{Q} 698))-7.707 * \operatorname{EXP}(-9 * \mathrm{LN}(1$

$0)) * \operatorname{EXP}(3 * \mathrm{LN}(\mathrm{Q} 698))+8.745 * \operatorname{EXP}(-6 * \mathrm{LN}(10)) * \operatorname{EXP}(2 * \mathrm{LN}(\mathrm{Q} 698))-4.215 * \operatorname{EXP}(-$ $3 * \mathrm{LN}(10)) * \mathrm{Q} 698+1.351$

;MAJOR CS RADIUS

Q641=9.403* $\operatorname{EXP}(-13 * \operatorname{LN}(10)) * \operatorname{EXP}(4 * \mathrm{LN}(\mathrm{Q} 698))-3.195 * \operatorname{EXP}(-9 * \mathrm{LN}(1$

$0)) * \operatorname{EXP}(3 * \operatorname{LN}(\mathrm{Q} 698))+4.023 * \operatorname{EXP}(-6 * \operatorname{LN}(10)) * \operatorname{EXP}(2 * \operatorname{LN}(\mathrm{Q} 698))-2.279 * \operatorname{EXP}(-$ $3 * \mathrm{LN}(10)) * \mathrm{Q} 698+0.711$

;MINOR CS RADIUS

$\mathrm{Q} 642=8.526 * \operatorname{EXP}(-13 * \mathrm{LN}(10)) * \operatorname{EXP}(4 * \mathrm{LN}(\mathrm{Q} 698))-2.752 * \operatorname{EXP}(-9 * \mathrm{LN}($ 10))* $\operatorname{EXP}(3 * \mathrm{LN}(\mathrm{Q} 698))+3.314 * \operatorname{EXP}(-6 * \mathrm{LN}(10)) * \operatorname{EXP}(2 * \mathrm{LN}(\mathrm{Q} 698))-1.86 * \mathrm{EXP}(-$ $3 * \mathrm{LN}(10)) * \mathrm{Q} 698+0.529$

;

;LATERAL MOVE PERCENTAGE

Q643=Q699/Q622

;MOVE DISTANCE

Q644=(Q641-Q619/2)*Q643

;DEPTH AT LATERAL POSITION

Q645=SQRT((1-((Q644*Q644)/(Q641*Q641)))*Q642*Q642)*Q690 ;

;MOVE POSITIONS

;RADIUS

Q650=Q640-Q644

$\mathrm{Q} 651=\mathrm{COS}(\mathrm{Q} 698) * \mathrm{Q} 650 \quad ; \mathrm{X} \mathrm{MOVE}$

$\mathrm{Q} 652=\mathrm{SIN}(\mathrm{Q} 698) * \mathrm{Q} 650 ; \mathrm{Y}$ MOVE

;

$\mathrm{X}(\mathrm{Q} 610+\mathrm{Q} 651) \mathrm{Y}(\mathrm{Q} 611+\mathrm{Q} 652 * \mathrm{Q} 623) \mathrm{Z}(\mathrm{Q} 612+\mathrm{Q} 645)$

$\mathrm{F}(\mathrm{Q} 630)$

Q698=Q698-Q621;CYCLE COUNT

FOR SPIRAL PROGRESSION

ENDWHILE

Q699=Q699+1 ;CYCLE COUNT FOR PASSES

ENDWHILE

F3

Z(Q612-20) ;SAFE Z

DWELL1

;

RETURN

CLOSE 


\section{Dover milling machine code gp2dcut.pmc}

For cutting out cavity machined with gp2dcav.pmc code.

\section{OPEN PROG 999 CLEAR}

;gp2dcav.pmc : For cutting out a partial ST guinea pig cavity without the axial ; component in plexiglass. Just under one turn is created using a $250 \mathrm{um}$ ball ;nosed endmill. A negative can be created in the cover so the two pieces ; can be glued together to form the cavity.

;

LIN

$\operatorname{ABS}(\mathrm{X}, \mathrm{Y}, \mathrm{Z})$

$\operatorname{FRAX}(\mathrm{X}, \mathrm{Y}, \mathrm{Z})$

Q600 $=1 \quad$;spiral direction $(1$ or -1$)$

;

;Start position at surface

$\mathrm{Q} 610=-54.436 \quad ; \mathrm{X}$ POSITION

Q611 $=-16.203+24 \quad ; Y$ POSITION

Q612 $=117.164 \quad$;Z SURF REF

;

Q619=2 ;TOOL DIAM

$\mathrm{Q} 620=1.962+.1 \quad$;radius of round

Q621 $=12 \quad$;length of handle

$\mathrm{Q} 622=1.601 \quad$;plexiglass thickness

$\mathrm{Q} 623=0.4 \quad$;depth of cut

$\mathrm{Q} 630=1.2 \quad$;FEED FOR CUTS

;

Q631=Q620+Q619/2 ;RADIUS TO DO CUT

;

F2

Z(Q612-20) ;Z SAFE

DWELL1

X(Q610) Y(Q611)

DWELL1

Z(Q612-1)

DWELL1

X(Q610-Q621-Q619/2) Y(Q611+Q631)

DWELL1

$\mathrm{F}(\mathrm{Q} 630)$

NORMAL K-1

Q699=Q623

;

WHILE(Q699!>(Q622+Q623))

Q640=Q612+Q699;DEPTH FOR CUT

F(Q630*.1) ;PLUNGE FEED 


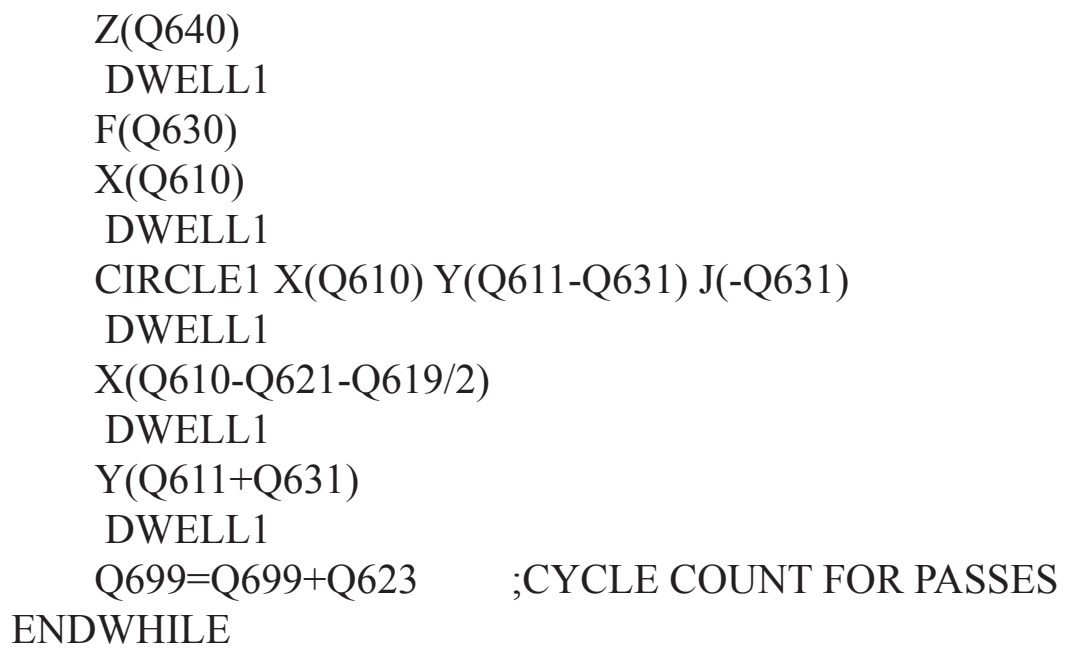

Dover milling machine code gpwind.pmc

For machining brass winding mandrels.

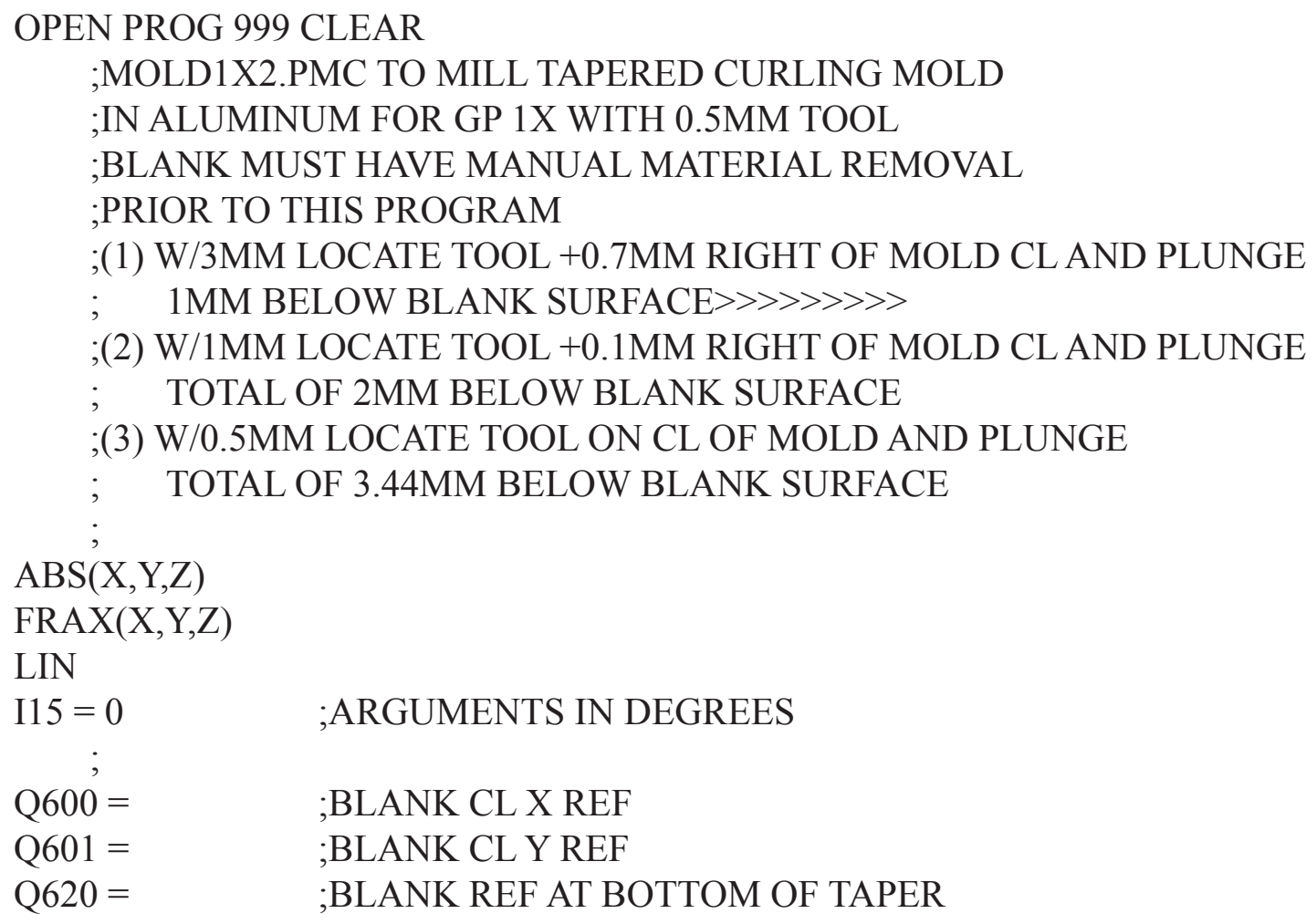




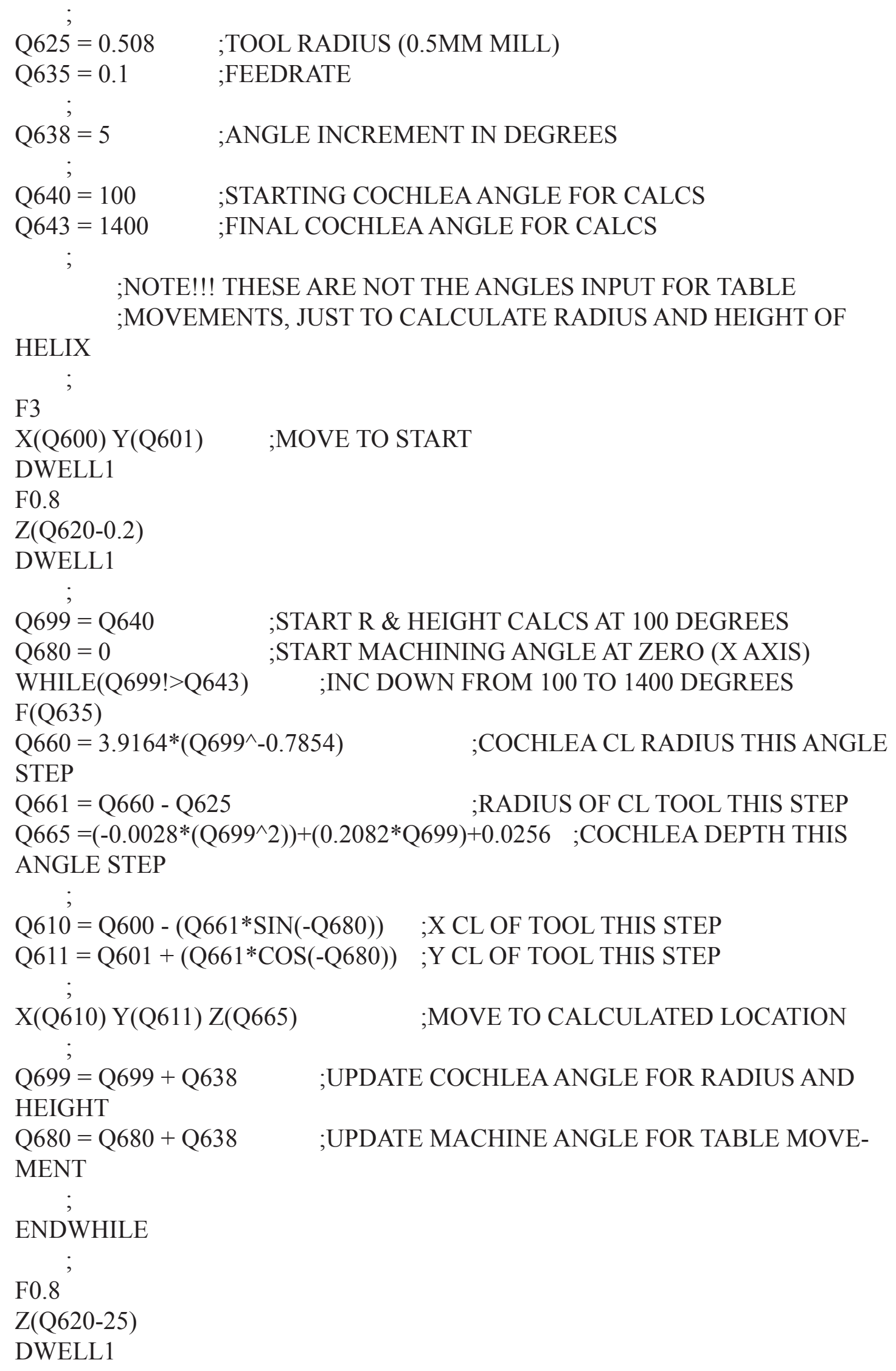




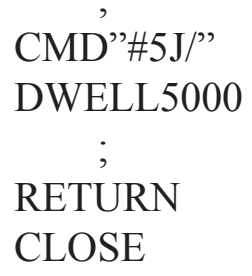

For machining brass foil protector backings for silicon probes. Please note that this program was modified from earlier code designed for another purpose and therefore contains superfluous coding.

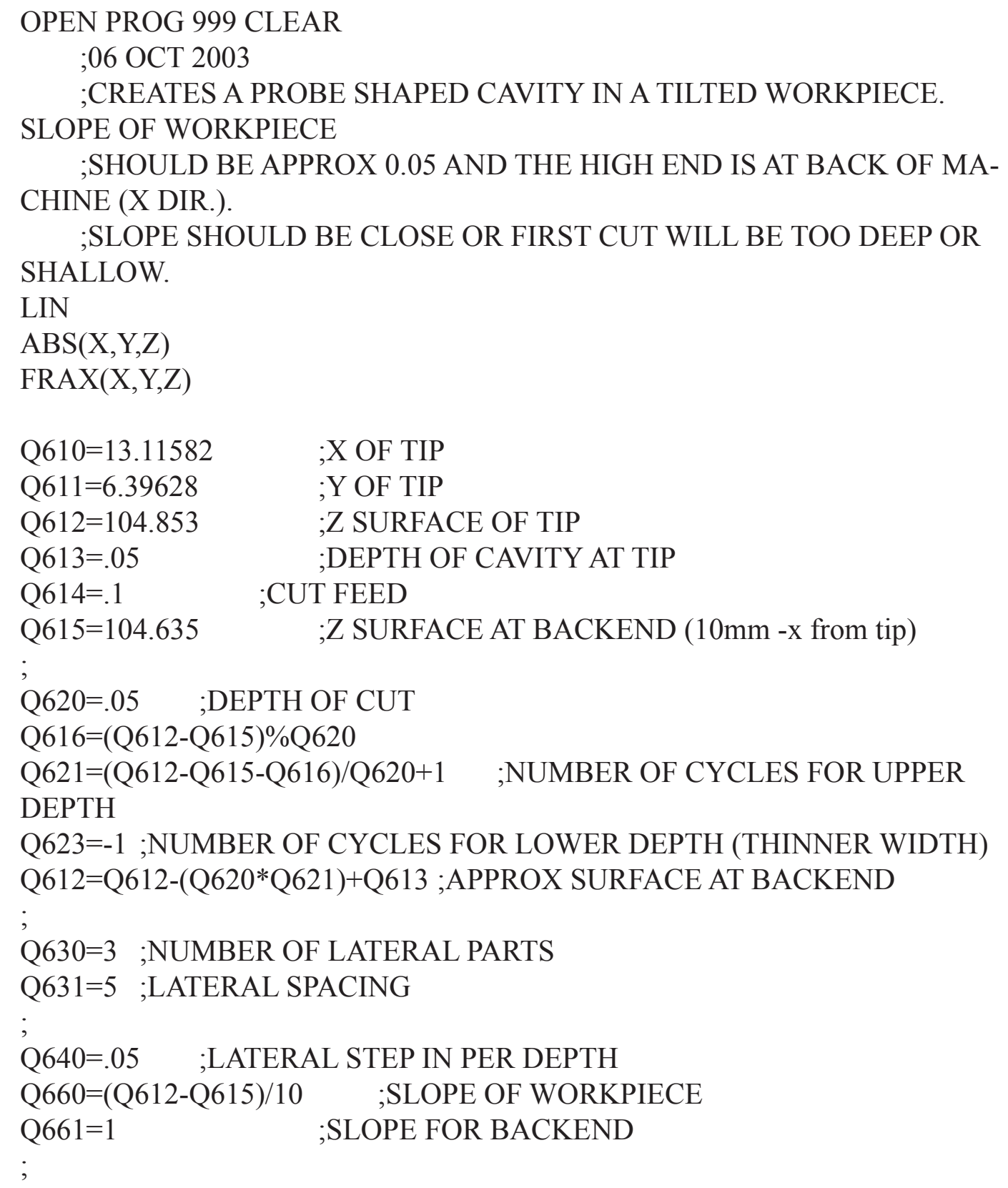




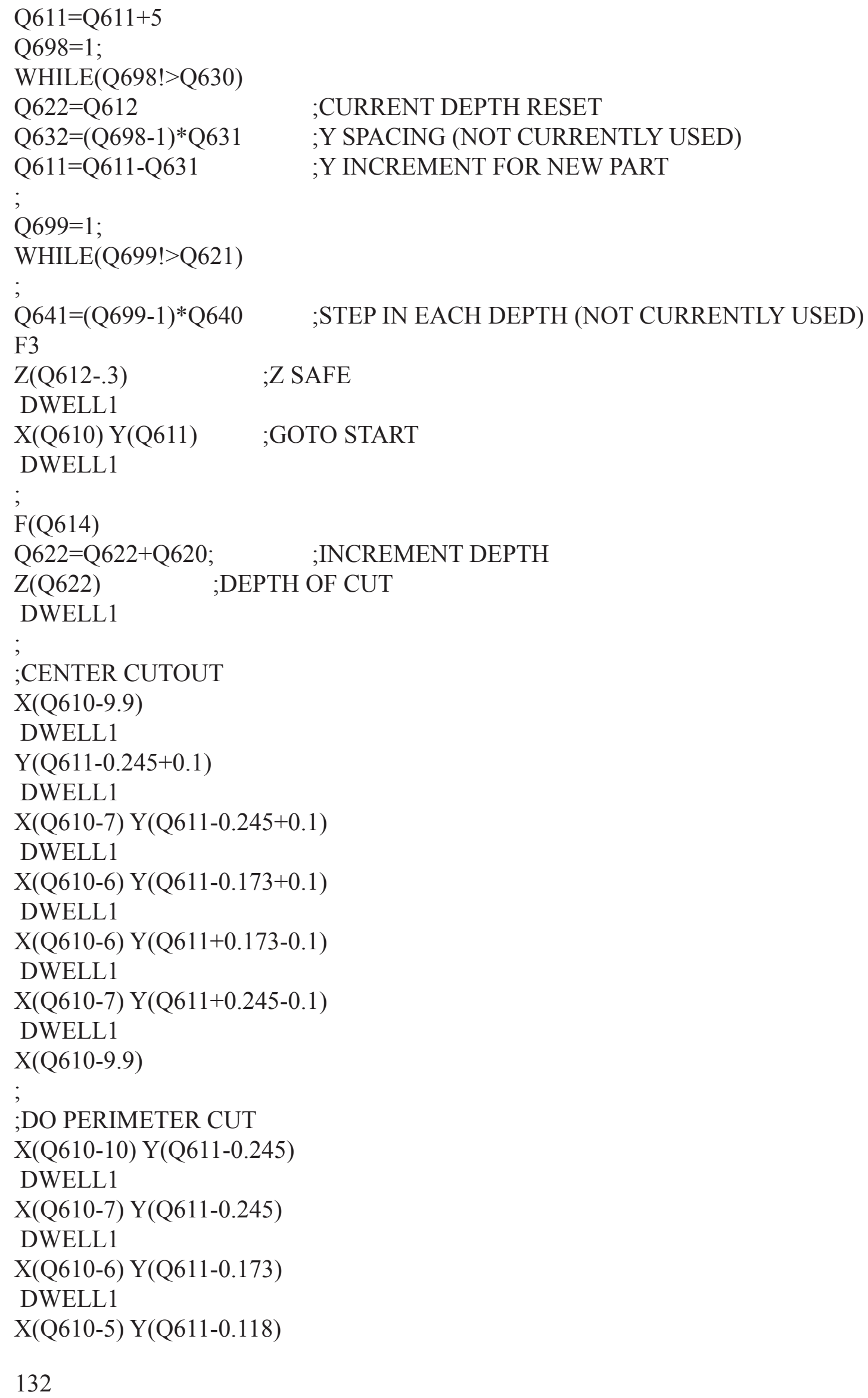


DWELL1

X(Q610-4) Y(Q611-0.075)

DWELL1

X(Q610-3) Y(Q611-0.045)

DWELL1

X(Q610-2) Y(Q611-0.025)

DWELL1

$\mathrm{X}(\mathrm{Q} 610) \mathrm{Y}(\mathrm{Q} 611)$

DWELL1

$\mathrm{X}(\mathrm{Q} 610-2) \mathrm{Y}(\mathrm{Q} 611+0.025)$

DWELL1

X(Q610-3) Y(Q611+0.045)

DWELL1

X(Q610-4) Y(Q611+0.075)

DWELL1

X(Q610-5) Y(Q611+0.118)

DWELL1

X(Q610-6) Y(Q611+0.173)

DWELL1

X(Q610-7) Y(Q611+0.245)

DWELL1

$\mathrm{X}(\mathrm{Q} 610-10) \mathrm{Y}(\mathrm{Q} 611+0.245)$

DWELL1

Y(Q611-0.245)

DWELL1

;

;

;Slope at backend

$\mathrm{Q} 662=\mathrm{Q} 622-\mathrm{Q} 612+.1 \quad$;CUT DISTANCE FOR SLOPE

$\mathrm{X}(\mathrm{Q} 610-10-\mathrm{Q} 662) \mathrm{Z}(\mathrm{Q} 622-(\mathrm{Q} 662 *(\mathrm{Q} 660+\mathrm{Q} 661))) \quad$;SLOPE CUT 1OF5

DWELL1

$\mathrm{X}(\mathrm{Q} 610-10)$

DWELL1

Y(Q611-0.195) Z(Q622)

DWELL1

X(Q610-10-Q662) Z(Q622-(Q662*(Q660+Q661))) ；SLOPE CUT 2OF5

DWELL1

$\mathrm{X}(\mathrm{Q} 610-10)$

DWELL1

Y(Q611-0.145) Z(Q622)

DWELL1

X(Q610-10-Q662) Z(Q622-(Q662*(Q660+Q661))) ；SLOPE CUT 3OF5

DWELL1

X(Q610-10)

DWELL1 







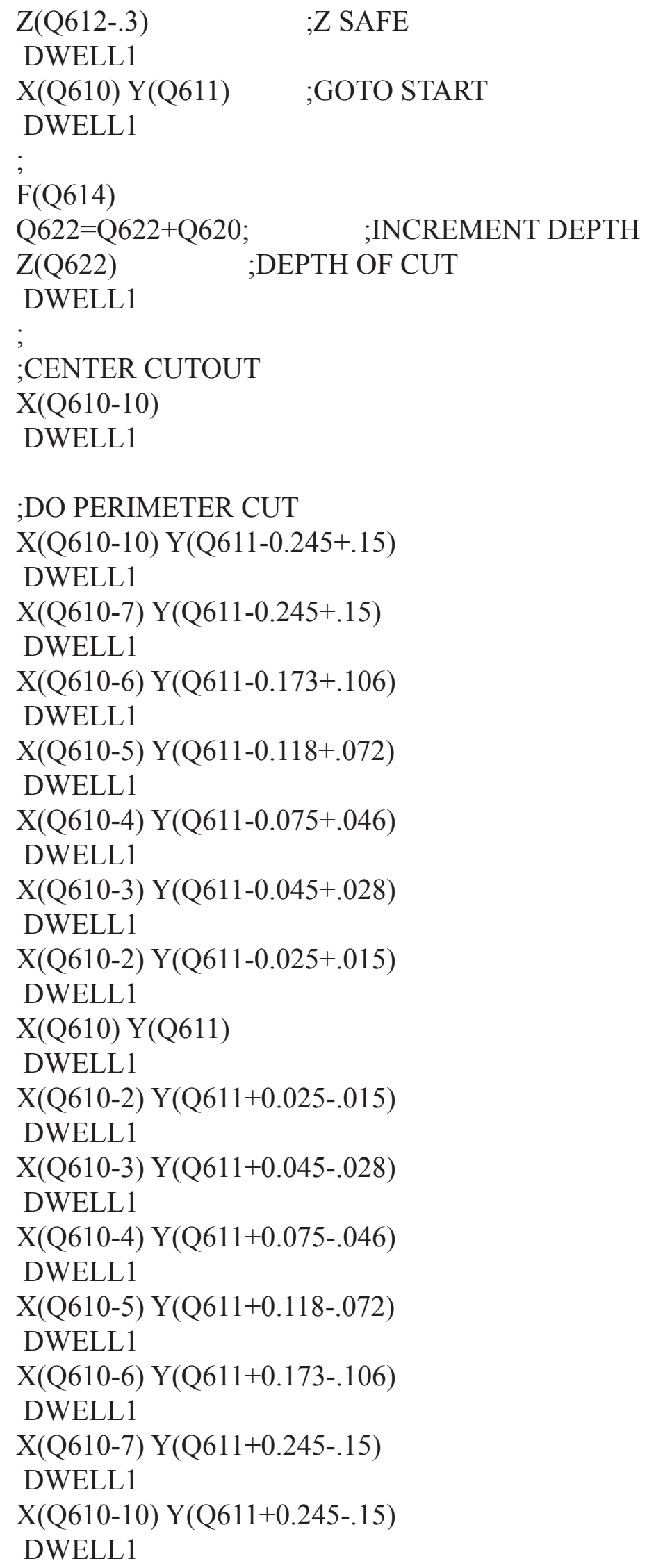


$\mathrm{Y}(\mathrm{Q} 611-0.245+.15)$

DWELL1

Q697=Q697+1

ENDWHILE

Q698=Q698+1

ENDWHILE

;SHUTDOWN

F3

Z(Q612-30)

CMD"\#5J/"

DWELL3000

CMD"\#5J/"

DWELL3000

CMD"\#5K"

DWELL3000

RETURN

CLOSE 


\section{Appendix C: $\quad$ Data from Chamber Actuation Expermients}

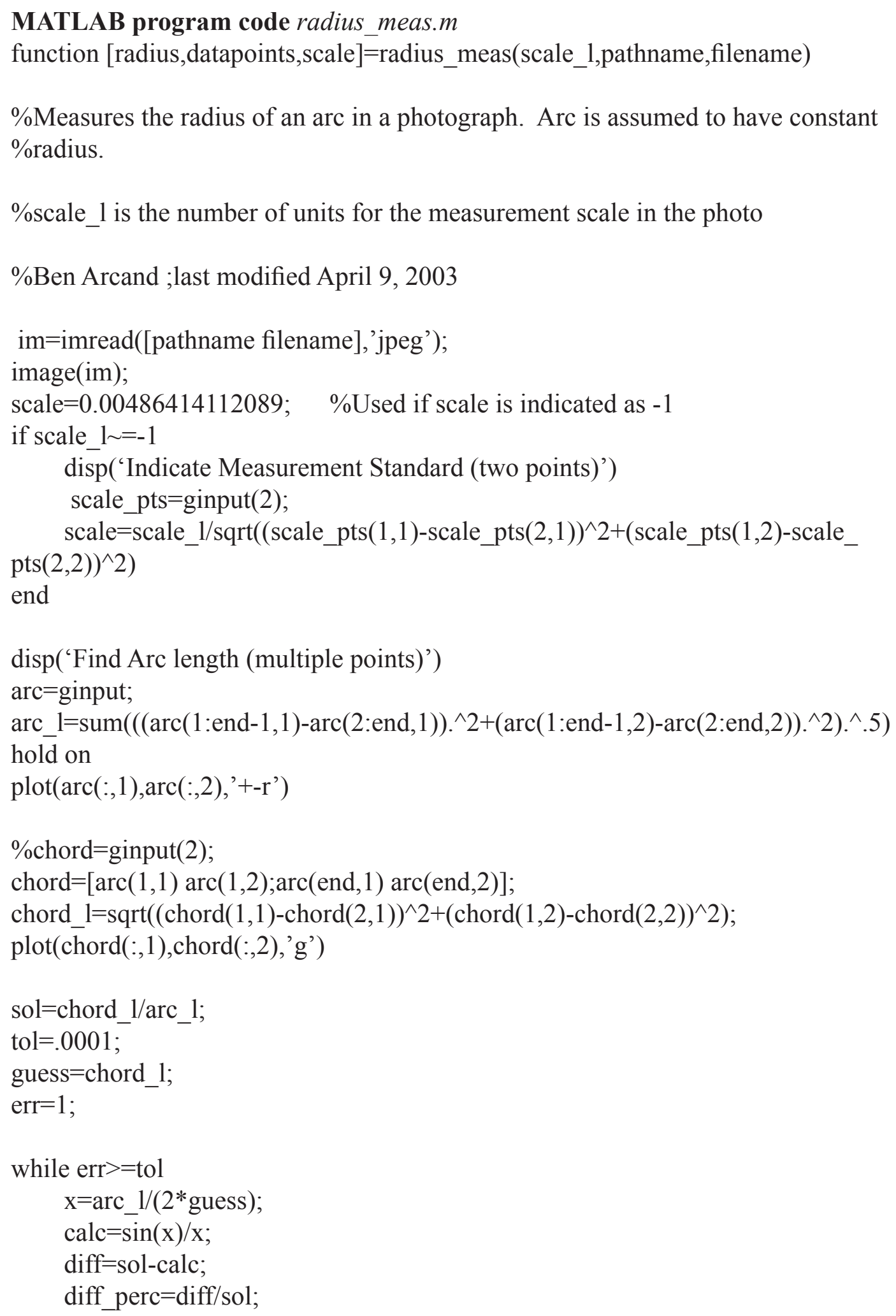


guess $=$ guess + guess $*$ diff_perc;

err=abs(diff);

end

$[\mathrm{R}]=$ findradfromchord(arc_1, chord_1);

disp(num2str(scale))

radius $=$ guess* ${ }^{*}$ scale;

datapoints $=$ arc;

radius $(2)=\mathrm{R} *$ scale;

datapoints(:,1)=datapoints(:,1)-arc(1,1);

datapoints(:,2)=datapoints(:,2)-datapoints(1,2);

datapoints $=$ datapoints* scale;

\%datapoints=mat2cell(datapoints);

disp('Datapoints:')

disp(num2str(datapoints))

$\operatorname{disp}($ ")

disp('Radius:')

disp(num2str(radius));

Chamber actuation measurements for a manufactured radius of $1 \mathrm{~mm}$ :

\begin{tabular}{|c|c|c|c|c|c|c|c|}
\hline Chamber & Pressure & $\begin{array}{c}\text { Radius } \\
\mathbf{1}\end{array}$ & $\begin{array}{c}\text { Radius } \\
\mathbf{2}\end{array}$ & $\begin{array}{c}\text { Radius } \\
\mathbf{3}\end{array}$ & $\begin{array}{c}\text { Radius } \\
\mathbf{4}\end{array}$ & $\begin{array}{c}\text { Radius } \\
\mathbf{5}\end{array}$ & Avg. Rad. \\
\hline Number & $\mathbf{p s i}$ & $\mathbf{m m}$ & $\mathbf{m m}$ & $\mathbf{m m}$ & $\mathbf{m m}$ & $\mathbf{m m}$ & $\mathbf{m m}$ \\
\hline 1 & 0.0 & 1.016 & 0.976 & 0.983 & 0.999 & 0.964 & 0.988 \\
\hline 1 & 7.5 & 2.067 & 2.175 & 2.182 & 2.262 & 2.301 & 2.198 \\
\hline 1 & 11.5 & 4.077 & 3.862 & 4.182 & 3.811 & 4.113 & 4.009 \\
\hline 1 & 16.1 & 6.957 & 6.034 & 6.172 & 6.195 & 6.476 & 6.367 \\
\hline 1 & 20.4 & 7.974 & 8.952 & 9.525 & 8.721 & 8.886 & 8.812 \\
\hline 1 & 25.4 & 11.363 & 9.775 & 12.134 & 8.172 & 9.236 & 10.136 \\
\hline 1 & 30.2 & 11.459 & 11.668 & 12.877 & 12.900 & 13.481 & 12.477 \\
\hline 2 & 0.0 & 0.959 & 0.959 & 0.959 & 0.933 & 0.954 & 0.953 \\
\hline 2 & 4.8 & 0.974 & 0.968 & 0.943 & 0.952 & 0.940 & 0.955 \\
\hline 2 & 10.0 & 1.378 & 1.461 & 1.367 & 1.387 & 1.405 & 1.400 \\
\hline 2 & 15.0 & 2.622 & 2.635 & 2.617 & 2.882 & 2.823 & 2.716 \\
\hline 2 & 20.0 & 4.447 & 4.265 & 4.624 & 4.419 & 4.231 & 4.397 \\
\hline 2 & 24.8 & 6.205 & 6.237 & 5.571 & 6.083 & 5.988 & 6.017 \\
\hline 2 & 29.8 & 9.942 & 7.902 & 7.921 & 7.669 & 8.469 & 8.380 \\
\hline 3 & 0.0 & 0.981 & 0.961 & 0.971 & 0.979 & 0.979 & 0.974 \\
\hline 3 & 4.8 & 0.969 & 0.957 & 0.990 & 0.953 & 0.977 & 0.969 \\
\hline 3 & 10.0 & 2.093 & 1.979 & 2.089 & 1.989 & 2.127 & 2.055 \\
\hline 3 & 14.8 & 3.161 & 3.621 & 3.709 & 3.475 & 3.576 & 3.508 \\
\hline
\end{tabular}




\begin{tabular}{|l|c|c|c|c|c|c|c|}
\hline 3 & 20.0 & 4.515 & 4.291 & 5.082 & 5.356 & 4.916 & 4.832 \\
\hline 3 & 25.0 & 7.577 & 6.896 & 7.001 & 8.370 & 7.436 & 7.456 \\
\hline 3 & 30.0 & 9.579 & 9.060 & 10.422 & 9.496 & 9.617 & 9.635 \\
\hline 4 & 0.0 & 0.638 & 0.627 & 0.618 & 0.658 & 0.628 & 0.634 \\
\hline 4 & 5.0 & 0.618 & 0.621 & 0.635 & 0.624 & 0.627 & 0.625 \\
\hline 4 & 10.0 & 0.623 & 0.628 & 0.625 & 0.660 & 0.621 & 0.632 \\
\hline 4 & 15.2 & 2.656 & 2.813 & 2.651 & 2.520 & 2.607 & 2.649 \\
\hline 4 & 19.8 & 3.689 & 4.612 & 4.073 & 3.708 & 4.940 & 4.205 \\
\hline 4 & 25.0 & 5.397 & 5.791 & 4.980 & 5.599 & 5.247 & 5.403 \\
\hline 4 & 29.8 & 6.126 & 6.841 & 7.768 & 10.583 & 6.128 & 7.489 \\
\hline 5 & 0.0 & 0.614 & 0.604 & 0.615 & 0.605 & 0.604 & 0.608 \\
\hline 5 & 5.0 & 0.609 & 0.600 & 0.598 & 0.609 & 0.617 & 0.607 \\
\hline 5 & 10.0 & 0.604 & 0.605 & 0.615 & 0.615 & 0.627 & 0.613 \\
\hline 5 & 14.9 & 1.948 & 1.926 & 1.705 & 1.822 & 1.879 & 1.856 \\
\hline 5 & 20.0 & 3.097 & 3.668 & 2.992 & 3.247 & 3.360 & 3.273 \\
\hline 5 & 25.0 & 5.357 & 5.622 & 5.363 & 5.341 & 5.254 & 5.387 \\
\hline 5 & 30.0 & 7.182 & 6.139 & 6.088 & 6.107 & 6.037 & 6.311 \\
\hline 6 & 0.0 & 0.628 & 0.620 & 0.621 & 0.627 & 0.605 & 0.620 \\
\hline 6 & 5.2 & 0.604 & 0.628 & 0.622 & 0.633 & 0.600 & 0.617 \\
\hline 6 & 10.0 & 0.638 & 0.629 & 0.596 & 0.632 & 0.633 & 0.626 \\
\hline 6 & 15.0 & 0.760 & 0.835 & 0.834 & 0.841 & 0.827 & 0.820 \\
\hline 6 & 20.0 & 2.617 & 2.391 & 2.435 & 2.400 & 2.486 & 2.466 \\
\hline 6 & 25.0 & 3.673 & 3.877 & 3.416 & 3.164 & 3.315 & 3.489 \\
\hline 6 & 29.2 & 4.496 & 4.638 & 4.201 & 5.720 & 4.899 & 4.791 \\
\hline
\end{tabular}

Chamber actuation measurements with pressure, post-pressure, and vacuum:

\begin{tabular}{|c|c|c|c|c|c|c|}
\hline \multicolumn{7}{|c|}{ Radius measurements for a Pressurized Chamber } \\
\hline Pressure & $\begin{array}{c}\text { Radius } \\
\mathbf{1}\end{array}$ & $\begin{array}{c}\text { Radius } \\
\mathbf{2}\end{array}$ & $\begin{array}{c}\text { Radius } \\
\mathbf{3}\end{array}$ & $\begin{array}{c}\text { Radius } \\
\mathbf{4}\end{array}$ & $\begin{array}{c}\text { Radius } \\
\mathbf{5}\end{array}$ & $\begin{array}{c}\text { Avg. } \\
\text { Rad. }\end{array}$ \\
\hline $\mathbf{p s i}$ & $\mathbf{m m}$ & $\mathbf{m m}$ & $\mathbf{m m}$ & $\mathbf{m m}$ & $\mathbf{m m}$ & $\mathbf{m m}$ \\
\hline 0 & 9.451 & 9.248 & 9.494 & 9.789 & 8.924 & 9.381 \\
\hline 2 & 10.792 & 10.875 & 13.716 & 14.596 & 12.037 & 12.403 \\
\hline 4 & 12.919 & 12.574 & 12.584 & 15.16 & 12.08 & 13.063 \\
\hline 6 & 16.469 & 14.939 & 16.617 & 17.443 & 14.649 & 16.023 \\
\hline 8 & 18.215 & 17.185 & 18.102 & 17.994 & 18.712 & 18.041 \\
\hline 10 & 27.453 & 26.695 & 27.357 & 27.173 & 25.945 & 26.924 \\
\hline
\end{tabular}




\begin{tabular}{|c|c|c|c|c|c|c|}
\hline 12 & 34.036 & 34.528 & 34.116 & 31.496 & 35.742 & 33.983 \\
\hline 15 & 38.467 & 40.492 & 38.102 & 41.837 & 38.96 & 39.571 \\
\hline \multicolumn{7}{|c|}{ Measurements Post-Actuation } \\
\hline 2 & 10.031 & 10.37 & 10.629 & 10.39 & 10.39 & 10.362 \\
\hline 4 & 11.06 & 10.756 & 10.864 & 11.152 & 9.766 & 10.719 \\
\hline 6 & 12.723 & 11.985 & 11.322 & 11.041 & 11.722 & 11.758 \\
\hline 8 & 12.363 & 12.482 & 13.315 & 14.679 & 14.489 & 13.465 \\
\hline 10 & 14.11 & 13.579 & 13.804 & 13.387 & 13.579 & 13.691 \\
\hline 12 & 14.87 & 15.078 & 15.367 & 15.881 & 15.838 & 15.406 \\
\hline 15 & 20.753 & 19.125 & 17.912 & 21.632 & 20.804 & 20.045 \\
\hline \multicolumn{7}{|c|}{$\begin{array}{l}\text { Measurements Post-Actuation with a Vacuum Applied to } \\
\text { Chamber }\end{array}$} \\
\hline 6 & 6.128 & 6.524 & 6.403 & 5.913 & 6.475 & 6.289 \\
\hline 8 & 6.368 & 6.529 & 6.639 & 6.190 & 6.465 & 6.438 \\
\hline 10 & 6.418 & 6.366 & 6.547 & 6.644 & 6.569 & 6.509 \\
\hline 12 & 6.733 & 7.108 & 6.879 & 6.756 & 6.659 & 6.827 \\
\hline 15 & 7.192 & 7.075 & 7.080 & 6.940 & 7.054 & 7.068 \\
\hline
\end{tabular}




\section{Appendix D: $\quad$ Programs for Modeling Bourdon Actuation of Chambers}

MATLAB program code $c s \_a b a q u s \_$inp.m

Program builds an ABAQUS input file for an FEM of a shell cross section swept along a constant radius arc.

function [end_pts,fixed_cs]=cs_abaqus_inp_inp(jobname,path,cs,R,degrees,P,prop,fir st_step,min_step)

$\%$ Abaqus scripting tool

\%units in microns

$\%$ cs-columner cs points $(\mathrm{x}, \mathrm{y})$ for the positive quadrant, order $\mathrm{x}=0->\mathrm{x}+$

$\%$ s.prop:(1)=E, (2)=Shear, (3)=nu, (4)=t

$\%$ degrees-angle in degrees

$\%$ P-pressure

$\%$ end pts $=$ end points to focus on for calculating radius

$\%$ fixed_cs=fixed end cross section points

file_ext='.inp';

$\mathrm{cs}=[\mathrm{cs} ;[\operatorname{cs}($ end-1:-1:1,1) $-\mathrm{cs}($ end-1:-1:1,2)]];

$\operatorname{cs}=[\operatorname{cs}(:, 2) \operatorname{cs}(:, 1)]$;

note1=['Jobname: ',jobname,'Radius: ',num2str(R),' Arc degrees:

',num2str(degrees),' Pressure: ',num2str(P),' thickness:',num2str(prop(4))];

datemade $=$ date;

note2 $=[$ 'Made by cs_change_shell_inp.m on ',datemade];

edge_size $=\operatorname{sum}\left(\operatorname{sqrt}\left((\operatorname{cs}(2\right.\right.$ :end, 1$)-\operatorname{cs}(1$ :end- 1,1$)) \cdot{ }^{\wedge} 2+(\operatorname{cs}(2$ :end, 2$)-\operatorname{cs}(1$ :end-

$1,2)) \cdot \wedge 2)) /($ length $(\mathrm{cs})-1)$;

num_steps $=1$;

max_inc $=10000$;

stabilize_factor $=0$;

ns $=$ round $\left(2 * \mathrm{pi}^{*} \mathrm{R} *(\right.$ degrees/360)/edge_size $) ; \quad$ \%number of sections

theta $=($ degrees $/ 180 *$ pi $) /$ ns;

$\mathrm{np}=$ length $(\mathrm{cs})$;

\%number of cross section points

$\mathrm{mp}=\operatorname{ceil}(\mathrm{np} / 2)$;

$\%$ middle point node number

f_nm=[cs(:,1)-R,cs(:,2),zeros(np,1),ones(np,1)];

cs_ni $=[(\operatorname{cs}(1$ :end $-1,1)+\operatorname{cs}(2$ :end, 1$)) / 2,(\operatorname{cs}(1:$ end $-1,2)+\operatorname{cs}(2$ :end, 2$)) / 2] ; \quad \%$ intermediate points in crosss section 
f_ni=[cs_ni(:,1)-R,cs_ni(:,2),zeros(length(cs_ni),1),ones(length(cs_ni),1)]; \%intermediate points inside cross section






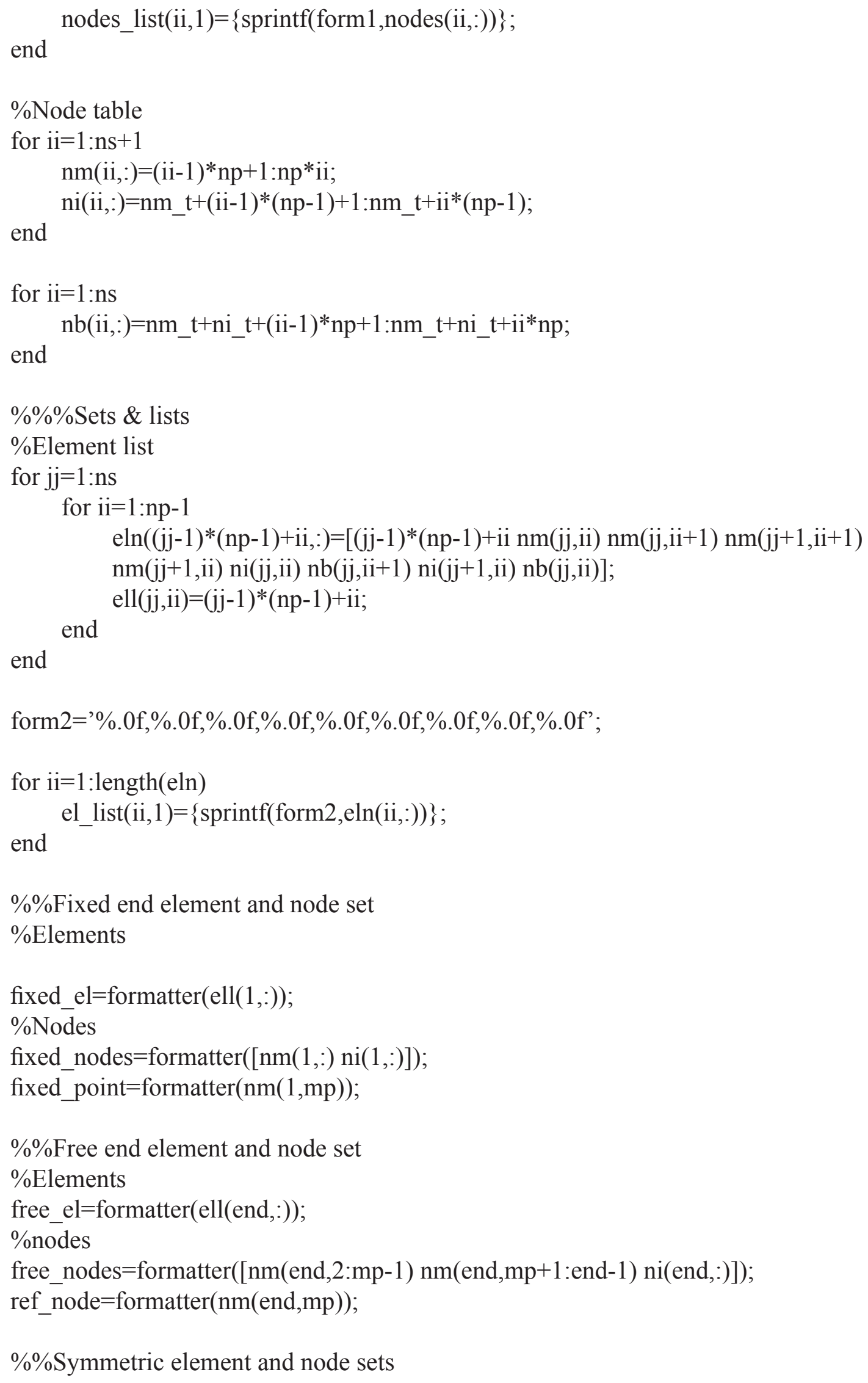




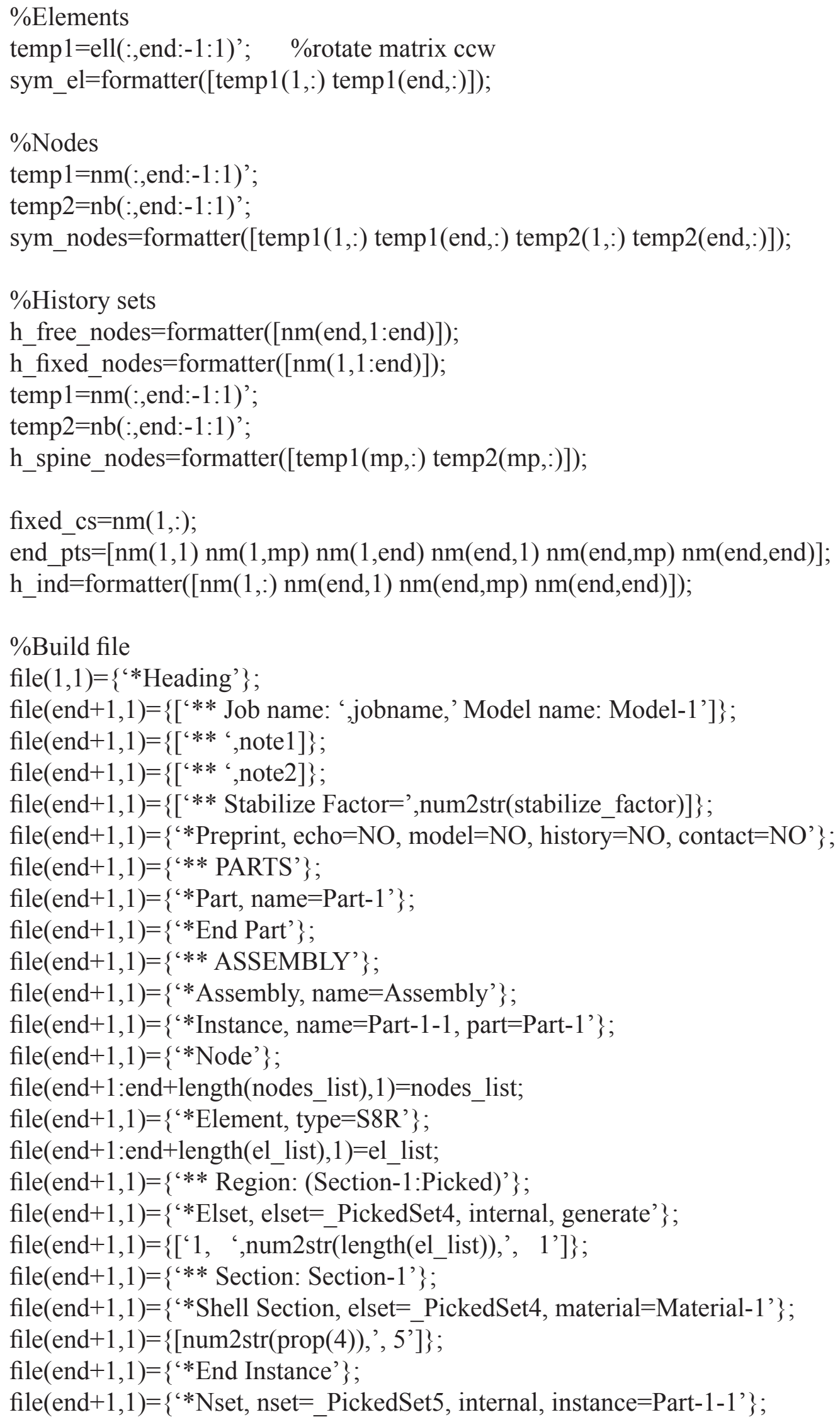


file(end +1 :end +length(fixed_nodes),1)=fixed_nodes;

file $($ end $+1,1)=\left\{{ }^{*}\right.$ Elset, elset $=$ PickedSet5, internal, instance $=$ Part-1-1' $\}$;

file(end +1 :end+length(fixed el $), 1)=$ fixed el;

file $($ end $+1,1)=\left\{{ }^{*} *\right.$ Nset, nset $=$ PickedSet6, internal, instance=Part-1-1' $\}$;

file $($ end $+1,1)=$ fixed_point;

file $($ end $+1,1)=\left\{{ }^{\prime} *\right.$ Nset, nset $=$ PickedSet7, internal, instance=Part-1-1' $\}$;

file(end +1 :end + length $($ sym_nodes $), 1)=$ sym_nodes;

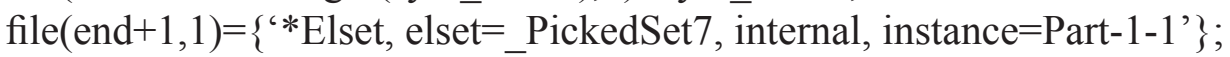

file (end +1 :end+length(sym_el $), 1)=$ sym_el;

file $($ end $+1,1)=\left\{{ }^{\prime} *\right.$ Nset, nset $=$ constrained, instance $=$ Part-1-1' $\}$;

file $($ end +1 :end + length(free_nodes $), 1)=$ free_nodes;

file $($ end $+1,1)=\left\{{ }^{*}\right.$ Elset, elset $=$ constrained, instance $=$ Part-1-1' $\}$;

file(end +1 :end + length(free_el), 1 )=free_el;

file $($ end $+1,1)=\left\{{ }^{* * N}\right.$ set, nset $=$ "ref node", instance=Part-1-1' $\}$;

file $($ end $+1,1)=$ ref_node;

file $($ end $+1,1)=\left\{{ }^{*}\right.$ Elset, elset $=\_$PickedSurf4_SNEG, internal, instance $=$Part-1-1, generate' $;$;

file $($ end $+1,1)=\left\{\left[{ }^{\prime} 1\right.\right.$, ',num $2 \operatorname{str}($ length(el_list $\left.\left.\left.)\right),, 1^{\prime}\right]\right\}$;

file $($ end $+1,1)=\left\{{ }^{\prime} *\right.$ Nset, nset $=$ h_free_nodes, instance $=$ Part-1-1' $\}$;

file $\left(\right.$ end +1 :end + length $\left(\mathrm{h} \_\right.$free_nodes $\left.), 1\right)=h$ _free_nodes;

file $($ end $+1,1)=\left\{{ }^{*} *\right.$ Nset, $n$ nset $=\bar{h} \_$fixed_nodes, instance=Part-1-1' $\}$;

file(end +1 :end + length(h_fixed_nodes $), 1)=h$ _fixed_nodes;

file $($ end $+1,1)=\left\{{ }^{*} *\right.$ Nset, nset $=\mathrm{h} \_$spine_nodes, instance $=$Part-1-1' $\}$;

file $($ end +1 :end + length $(\mathrm{h}$ _spine_nodes $), 1)=\mathrm{h}$ _spine_nodes;

file $($ end $+1,1)=\left\{{ }^{*}\right.$ N N set, $n$ set $=h \_$ind, instance $=$Part- $\left.-1-1\right\}$;

file $\left(\right.$ end +1 :end + length $\left(\mathrm{h} \_\right.$ind $\left.), \overline{1}\right)=\mathrm{h} \_$ind;

file $($ end $+1,1)=\left\{{ }^{*}\right.$ Surface, type $=$ ELEMENT, name $=$ PickedSurf4, internal' $\}$;

file $($ end $+1,1)=\left\{{ }^{6}\right.$ PickedSurf4_SNEG, SNEG' $\}$;

file $($ end $+1,1)=\{$ “*Kinematic Coupling, REF NODE="ref node"” $\}$;

file $($ end $+1,1)=\left\{{ }^{\prime}\right.$ constrained, 4,5 ' $\} ; \quad \% 4,5$ for $180-5,6$ for 90

file $($ end $+1,1)=\left\{{ }^{*}\right.$ End Assembly’ $\}$;

file $($ end $+1,1)=\left\{{ }^{* * *}\right.$ MATERIALS' $\}$;

file $($ end $+1,1)=\left\{{ }^{*} *\right.$ Material, name $=$ Material-1 $\left.{ }^{\prime}\right\}$;

file $($ end $+1,1)=\left\{{ }^{\prime *}\right.$ Elastic' $\}$;

file $($ end $+1,1)=\left\{\right.$ ‘ $^{2} 000,0.4$ ' $\}$;

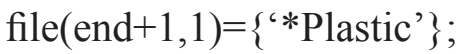

file $($ end $+1,1)=\left\{{ }^{\prime} 225,0 . '\right\}$;

file $($ end $+1,1)=\left\{{ }^{* * *}\right.$ BOUNDARY CONDITIONS $\}$;

file $($ end $+1,1)=\left\{{ }^{* *}\right.$ Name: BC-1 Type: Displacement/Rotation' $\}$;

file $($ end $+1,1)=\{$ '*Boundary' $\}$;

file $($ end $+1,1)=\left\{{ }^{6}\right.$ PickedSet5, 3, 3' $\}$;

file $($ end $+1,1)=\left\{{ }^{\prime}\right.$-PickedSet5, 4, 4' $\}$;

file $($ end $+1,1)=\left\{{ }^{\prime}\right.$ PickedSet5, 5, 5' $\}$; 


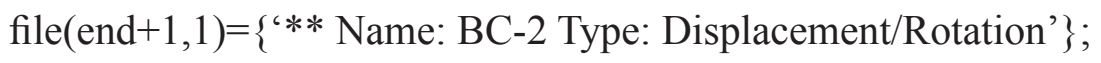

file $($ end $+1,1)=\left\{{ }^{*}\right.$ Boundary' $\}$;

file $($ end $+1,1)=\left\{{ }^{\prime}\right.$ PPickedSet6, 1, 1' $\}$;

file $($ end $+1,1)=\left\{{ }^{6}\right.$ PickedSet6, 3, 3' $\}$;

file $($ end $+1,1)=\left\{{ }^{\prime}\right.$ PickedSet6, 4, 4' $\}$;

file $($ end $+1,1)=\left\{{ }^{6}\right.$ PickedSet6, 5, 5' $\}$;

file $($ end $+1,1)=\left\{{ }^{\prime}\right.$ PPickedSet6, 6, 6' $\}$;

file $($ end $+1,1)=\left\{{ }^{6 * *}\right.$ Name: BC-3 Type: Symmetry/Antisymmetry/Encastre' $\}$;

file $($ end $+1,1)=\left\{{ }^{*}\right.$ Boundary' $\}$;

file $($ end $+1,1)=\{$ '_PickedSet7, YSYMM' $\}$;

for $\mathrm{ii}=1$ :num_steps



file $($ end $+1,1)=\left\{\left[{ }^{* * *}\right.\right.$ STEP: Step-', num $2 \operatorname{str}($ ii) $\left.\left.)\right]\right\}$;

file $($ end $+1,1)=\left\{\left[{ }^{\prime *}\right.\right.$ Step, name=Step-',num2str(ii),', nlgeom=YES',',

inc=',num $2 \operatorname{str}\left(\max \_\right.$inc $\left.\left.)\right]\right\}$;

if stabilize factor $==0$

file $($ end $+1,1)=\left\{\left[{ }^{‘ *}\right.\right.$ Static' $\left.]\right\}$;

else

file $($ end $+1,1)=\left\{\left[{ }^{*} *\right.\right.$ Static, Stabilize, Factor=',num2str(stabilize_factor) $\left.]\right\}$;

end

file $($ end $+1,1)=\left\{\left[\right.\right.$ num 2 str(first_step),, $1 .,{ }^{\prime}$, num $\left.\left.2 \operatorname{str}\left(\min \_s t e p\right), ', 1 . '\right]\right\} ; \%$ min

step* $10^{\wedge}$ (num_steps-ii)

file $($ end $+1,1)=\left\{{ }^{* * *} \operatorname{LOADS}^{\prime}\right\}$;

file $($ end $+1,1)=\left\{{ }^{* *}\right.$ Name: Load-1 Type: Pressure' $\}$;

file $($ end $+1,1)=\{$ '*Dsload' $\}$;

file $($ end $+1,1)=\left\{\left[{ }^{6}\right.\right.$ _PickedSurf4, P, ', num2str(P*ii $\left.\left.)\right]\right\}$;

$\%$ History Output

file $($ end $+1,1)=\{[$ ‘** OUTPUT REQUESTS'] $\}$;

file $($ end $+1,1)=\left\{\left[{ }^{*} *\right.\right.$ Restart, write, frequency $\left.\left.=1^{\prime}\right]\right\}$;

file $($ end $+1,1)=\left\{\left[{ }^{*} * *\right.\right.$ FIELD OUTPUT: F-Output-1'] $\}$;

file $($ end $+1,1)=\left\{\left[{ }^{*}\right.\right.$ Output, field, variable $=$ PRESELECT'] $\left.]\right\}$;

file $($ end $+1,1)=\left\{\left[{ }^{* * *}\right.\right.$ HISTORY OUTPUT: H-Output-1'] $]$;

file $($ end $+1,1)=\left\{\left[{ }^{* *}\right.\right.$ Output, history, variable=PRESELECT' $\left.]\right\}$;

$\%$ Free End History

$\%$ file $($ end $+1,1)=\left\{\left[{ }^{* * *}\right.\right.$ HISTORY OUTPUT: Free End History'] $\}$;

$\%$ file $($ end $+1,1)=\left\{\left[{ }^{*} *\right.\right.$ Output, history'] $\}$;

$\%$ file $($ end $+1,1)=\{[$ '*Node Output, nset $=$ h_free_nodes' $]\}$;

$\%$ file $($ end $+1,1)=\left\{\left[{ }^{\circ} \mathrm{COOR} 1, \mathrm{COOR} 2, \mathrm{COOR}{ }^{\prime}\right]\right\}$;

$\%$ Fixed End History

$\%$ file $($ end $+1,1)=\left\{\left[{ }^{* * *}\right.\right.$ HISTORY OUTPUT: Fixed End History'] $\}$;

$\%$ file $($ end $+1,1)=\{[$ '* Output, history'] $\}$;

$\%$ file $($ end $+1,1)=\left\{\left[{ }^{*} *\right.\right.$ Node Output, nset $=$ h_fixed_nodes' $\left.]\right\}$;

$\%$ file $($ end $+1,1)=\left\{\left[{ }^{\prime} C O O R 1\right.\right.$, COOR2, COOR3'] $\}$; 
file $($ end $+1,1)=\left\{{ }^{\prime *}\right.$ El Print, freq=999999' $\}$;

file $($ end $+1,1)=\left\{{ }^{*}\right.$ Node Print, freq $=999999$ ' $\}$;

file $($ end $+1,1)=\left\{{ }^{*}\right.$ NODE FILE, nset $=h \_$ind,freq $\left.=1 '\right\}$;

file $($ end $+1,1)=\{$ 'coord' $\}$;

file $($ end $+1,1)=\left\{{ }^{* *}\right.$ End Step' $\}$;

end

$\%$ Assemble input file

dlmwrite([path,jobname,file_ext],file(1),'delimiter',','newline','pc');

for $\mathrm{ii}=2$ :length(file)

dlmwrite([path,jobname,file_ext],file(ii), '-append','delimiter',','newline','pc');

end

disp('Input file written')

out $=1$;

return

MATLAB program code read_cs.m

Program reads the ABAQUS results file for deformed coordinates from the FEM created by cs_abaqus_inp.m.

function [R,cs,step] $=$ read_cs(jobname,path,end_pts,fixed_cs)

$\%$ Reads abaqus results for cs_change_shell_fil input

$\%$ Outputs radius $(\mathrm{R})$ and the new cross section points (cs) for each step

\%time (step)

fid=fopen([path,jobname,'.fil'] $])$;

fread(fid, 1 ,'int32');

$\mathrm{w}=$ fread(fid,'int64');

fclose(fid);

fid=fopen([path,jobname,'.fil'] $])$;

fread(fid,1,'int32');

$\mathrm{f}=$ fread(fid,'double');

fclose(fid);

i_org=find $(\mathrm{w}==1901) ; \%$ Indices for origional coordinates record number

i_def=find $(\mathrm{w}==107) ; \%$ Indices for deformed coordinates record number

i_p1 $=$ find $(w($ i_def +1$)==$ end pts $(1))$;

i_p11 $=$ find $\left(w\left(\bar{i} \_\right.\right.$def +1$)==$ end $\_$ts $\left.(2)\right)$;

i_p21=find $\left(w(\right.$ i_def +1$)==$ end $\_$ts $\left.(3)\right)$;

i_p232=find $(w($ i_def +1$)==$ end_pts $(4))$;

i_p242=find $\left(\mathrm{w}\left(\mathrm{i} \_\mathrm{def}+1\right)==\right.$ end_pts $\left.(5)\right)$; 


\section{MATLAB program code $P$ _diff.m}

Program calculates the distributed load factor resulting from a pressure differential between the inside and outside walls of the chamber given a cross section, a radius and a pressure. The load factor multiplied by the applied pressure gives the distributed load.

function [dist_load_factor] $=\mathrm{P} \_$diff( $(\mathrm{n}, \mathrm{R})$

$\%$ Assumes $\mathrm{y}=0$ as the neutral axis

$\%$ n-xy coordinates of the cross section

$\% \mathrm{R}$-radius of the chamber curvature

theta $=\operatorname{atan}(\operatorname{abs}((\mathrm{n}(2:$ end,2)-n(1:end-1,2))./(n(2:end,1)-n(1:end-1,1))) ;

element_length $=\operatorname{sqrt}((\mathrm{n}(2$ :end,2)-n(1:end-1,2)).^2+(n(2:end,1)-n(1:end-1,2)).^2);

$\mathrm{a}=\operatorname{abs}(\mathrm{n}(2$ :end,1)-n(1:end-1,1)); \%Length in $\mathrm{x}$ direction

$\mathrm{l}=\mathrm{abs}(\mathrm{n}(\mathrm{end}, 1)-\mathrm{n}(1,1)) ; \quad \%$ total length

partial $=\mathrm{a} / \mathrm{l}$;

y_cmpt $=\sin ($ theta $)$

$\mathrm{dy}=\operatorname{abs}(\mathrm{n}(2: \mathrm{end}, 2)-\mathrm{n}(1:$ end-1,2))/2; \%midpoint for element

radial_cmpt $=2 * \mathrm{dy} ; \% . /(\mathrm{R}+\mathrm{dy})$;

dist_load_factor=sum(partial.*radial_cmpt.*y_cmpt);

MATLAB program code $c l$ abaqus_inp.m

Program builds an ABAQUS input file for an FEM of a beam deflection with an arbitrarily defined cross section and a distributed load.

function [out]=beam3d_inp(jobname,path,n,s,first_step,min_step,distributedload)

file_ext='.inp';

n_el=length(n)-1;

[rows num_sect] $=$ size(s.prop);

$\%$ Build element/node tables

$\% \%$ Node Set

for ii $=1: \mathrm{n}$ el +1

n_set(ii,1) $=\{[$ num2str(ii),', ',num2str(n(ii,1)),', ',num2str(n(ii,2)), , ',num2str(n(ii,3))]\};

end

$\% \%$ Element Set

for ii $=1:$ n_el

el_set(ii,1) $=\{[$ num2str(ii),', ',num2str(ii),', ',num2str(ii+1) $]\} ;$

end

$\% * * * * *$ Build Input File***** 
file $(1,1)=\left\{{ }^{\prime *}\right.$ Heading' $\}$;

file $($ end $+1,1)=\left\{\left[{ }^{* * *}\right.\right.$ Job name: ',jobname,' Model name: Model-1'] $\left.]\right\}$;

file $($ end $+1,1)=\left\{{ }^{*} *\right.$ Preprint, echo $=\mathrm{NO}$, model $=\mathrm{NO}$, history $=\mathrm{NO}$, contact $=\mathrm{NO}$ ' $\}$;

file $($ end $+1,1)=\{$ *** PARTS' $\}$;

file $($ end $+1,1)=\left\{{ }^{\prime} *\right.$ Part, name $=$ Part $\left.-1 '\right\}$;

file $($ end $+1,1)=\left\{{ }^{*}\right.$ End Part' $\}$;

file $($ end $+1,1)=\left\{{ }^{*} *\right.$ ASSEMBLY' $\}$;

file $($ end $+1,1)=\left\{{ }^{*}\right.$ Assembly, name $=$ Assembly' $\}$;

file $($ end $+1,1)=\left\{{ }^{*}\right.$ Instance, name $=$ Part-1-1, part $=$ Part-1' $\}$;

file $($ end $+1,1)=\{$ '*Node' $\}$;

file(end +1 :end + length(n_set $), 1)=n \_s e t ;$

file $($ end $+1,1)=\left\{{ }^{*}\right.$ Element, type $\left.=B 31 '\right\}$;

file(end +1 :end+length(el_set),1)=el_set;

for $\mathrm{ii}=1$ :num_sect

file(end $+1,1)=\left\{\left[{ }^{‘ * *}\right.\right.$ Region: (Section-', num2str(ii),' :Picked), (Beam Orientation:

Picked)']\};

file $($ end $+1,1)=\left\{\left[{ }^{*} *\right.\right.$ Elset, elset=_Sect',num2str(ii),', internal, generate' $\left.]\right\}$;

file $($ end $+1,1)=\{[$ num $2 \operatorname{str}($ s.nsect(ii)), , ',num $2 \operatorname{str}($ s.nsect(ii+1)), , ',num $2 \operatorname{str}(\mathrm{s}$. nsect(ii))]\};

file $($ end $+1,1)=\left\{\left[{ }^{‘ * *}\right.\right.$ Section: Section-', num2str(ii),' Profile: Profile-1'] $\}$;

file $($ end $+1,1)=\left\{\left[{ }^{6 *}\right.\right.$ Beam General Section, elset $=$ Sect', num2str(ii),, , poisson $=$ ',num2str(s.prop(3,ii)),', section=ARBITRARY'] $\}$;

file $($ end $+1,1)=\{[$ num2str(length $($ s.coord(:,:;ii) $)-1),$, ', ,num $2 \operatorname{str}(\operatorname{s.coord}(1,1$, ii $))$, , ',num2str(s.coord(1,2,ii)),', ',num2str(s.coord(2,1,ii)),, ',num2str(s.

coord(2,2,ii)),', ',num2str(s.coord(2,3,ii))]\};

for $\mathrm{kk}=3:$ length $(\mathrm{s} . \operatorname{coord}(:, ;, \mathrm{ii}))$

file $($ end $+1,1)=\{[$ num2str(s.coord $(k k, 1, i i)),$, ',num2str(s.coord $(k k, 2, i i))$, ,

',num2str(s.coord(kk,3,ii))]\};

end

file $($ end $+1,1)=\left\{{ }^{\prime} 0 ., 0 ., 1 . ’\right\}$;

file $($ end $+1,1)=\left\{[\right.$ num2str(s.prop $(1, \mathrm{ii})),{ }^{\prime}$, ', $\left.\left.\operatorname{num} 2 \operatorname{str}(\operatorname{s.prop}(2, \mathrm{ii}))\right]\right\}$;

end

file $($ end $+1,1)=\left\{{ }^{*}\right.$ End Instance' $\}$;

file $($ end $+1,1)=\left\{{ }^{\prime} *\right.$ Nset, nset $=$ EndPoint1, internal, instance $=$ Part-1-1' $\}$;

file $($ end $+1,1)=\left\{{ }^{\prime} 1, '\right\}$;

file $($ end $+1,1)=\left\{{ }^{\prime *}\right.$ Nset, nset $=$ AllSet, internal, instance=Part-1-1, generate' $\}$;

file $($ end $+1,1)=\left\{\left[{ }^{\prime} 1\right.\right.$, ', num 2 str(n_el+1),', 1'] $\}$;

file $($ end $+1,1)=\left\{{ }^{*}\right.$ Elset, elset $=$ A AllSet, internal, instance $=$ Part-1-1, generate' $\}$;

file $($ end $+1,1)=\left\{\left[{ }^{\prime} 1\right.\right.$, ', num 2 str(n_el),', 1'] $]$;

file $($ end $+1,1)=\left\{{ }^{*}\right.$ End Assembly' $\}$;

$\mathrm{ii}=1$; 


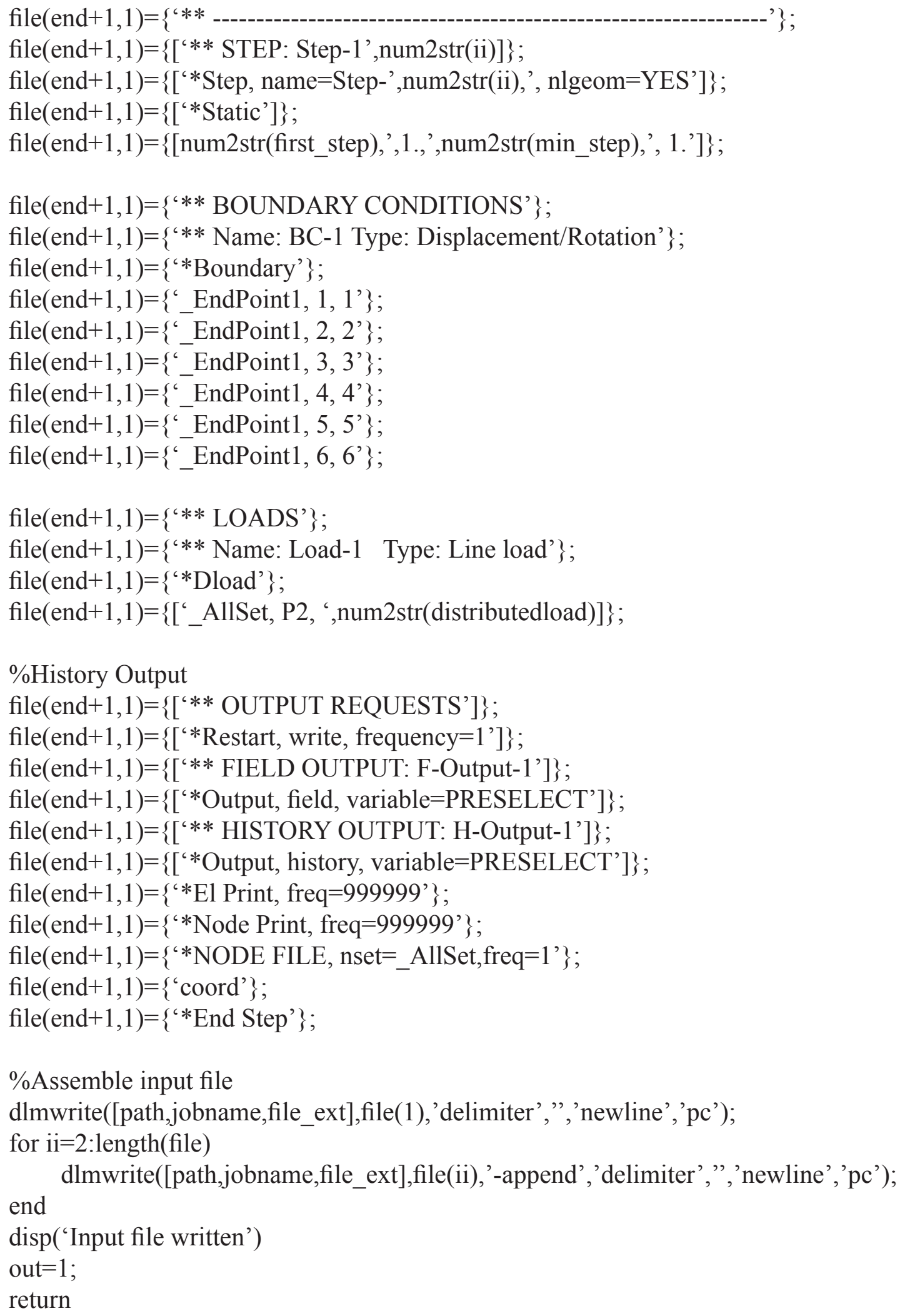


MATLAB program code read_cl.m

Program reads the ABAQUS results file for deformed coordinates from the FEM created by cl_abaqus_inp.m.

function [dat $\mathrm{w}$ cind] $=$ read_cl(path,filename)

$\%$ Read Abaqus *.fil only for coordinate values

A.diag $(1)=\{[$ 'File: ',path,filename $]\}$;

numtypes $=\{$ 'int64','double' $\}$;

for ii $=1$ :length(numtypes)

fid=fopen([path filename]);

fread(fid, 1,'int32'); \%Leading half-word

$\mathrm{w}(:, \mathrm{ii})=$ fread(fid,cell2mat(numtypes(ii))); \%record words

fclose(fid);

end

cind.org=find( $\mathrm{w}==1901)$; \% Indices for origional coordinates record number

cind.def $=$ find $(w==107) ; \%$ Indices for deformed coordinates record number

num_N=length(cind.org);

num_steps $=$ length(cind.def)/num_N;

dat.coord(:, $\mathrm{w}($ cind.org $+1,1), 1)=[\mathrm{w}($ cind.org $+2,2) \mathrm{w}($ cind.org $+3,2) \mathrm{w}($ cind.org $+4,2)]$;

dat.coord $(1: 3,1$ :num_N,2:num_steps +1$)=\operatorname{reshape}([\mathrm{w}($ cind.def $+2,2) \mathrm{w}(\operatorname{cind} . d e f+3,2)$

w(cind.def $+4,2)]$ ',3,num_N,num_steps);

dat.step $=\left[0 \mathrm{w}(\text { find }(\mathrm{w}(:, 1)==2000)+1,2)^{\prime}\right]$;

\section{MATLAB program code formatter.m}

Utility function program used by cs_abaqus_inp.m and $c l \_a b a q u s \_i n p . m$ to format long lists of numbers into rows of 16 deliminated by comas.

function [out] $=$ formatter(in)

$\mathrm{L}=16$;

if length(in) $<16$

$\mathrm{L}=$ length(in);

end

nrows=floor(length(in)/L);

for $\mathrm{ii}=1$ :nrows +1

start $=($ ii -1$) * \mathrm{~L}+1$;

addrow $=0$;

tmp2=";

if $\mathrm{ii}==$ nrows +1 





Appendix E: Interferometer Plots of a Polyimide Device

Measurements from the port area:



Contour plot of the port area:

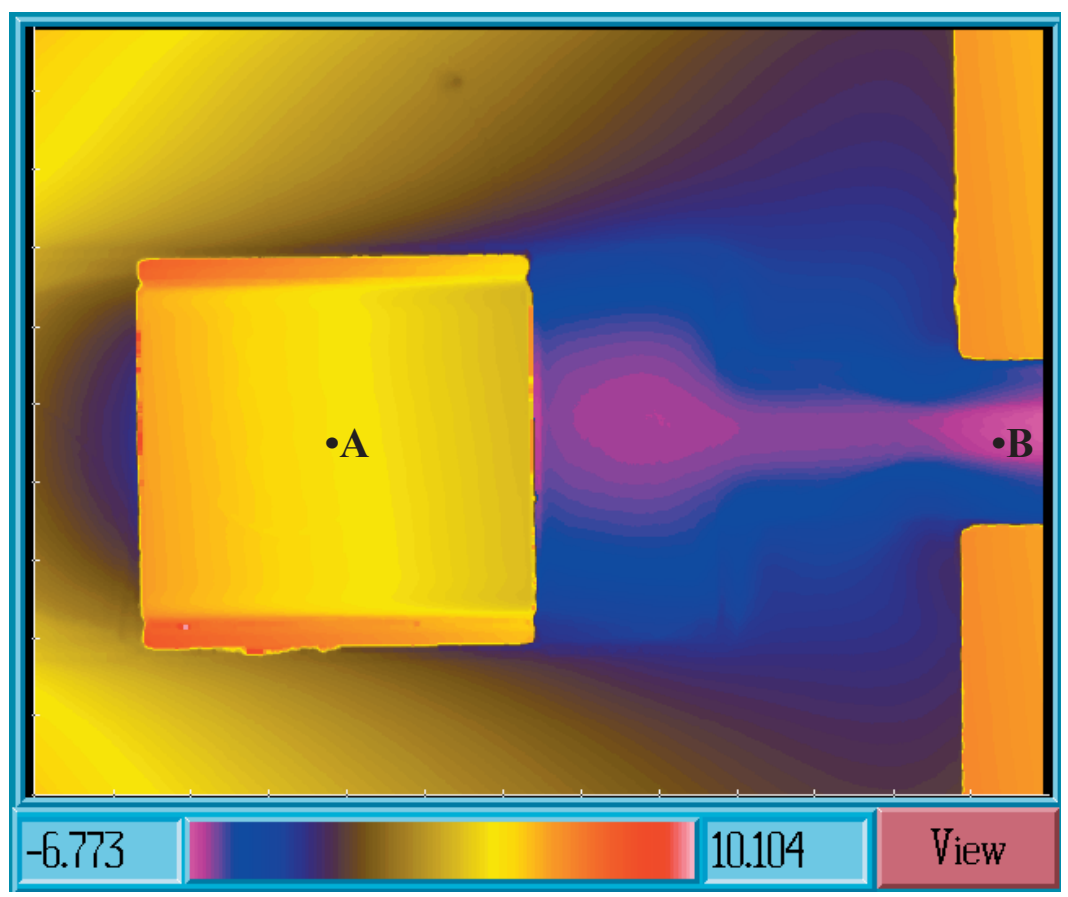


Plot of height through port center (Point A on port contour plot):

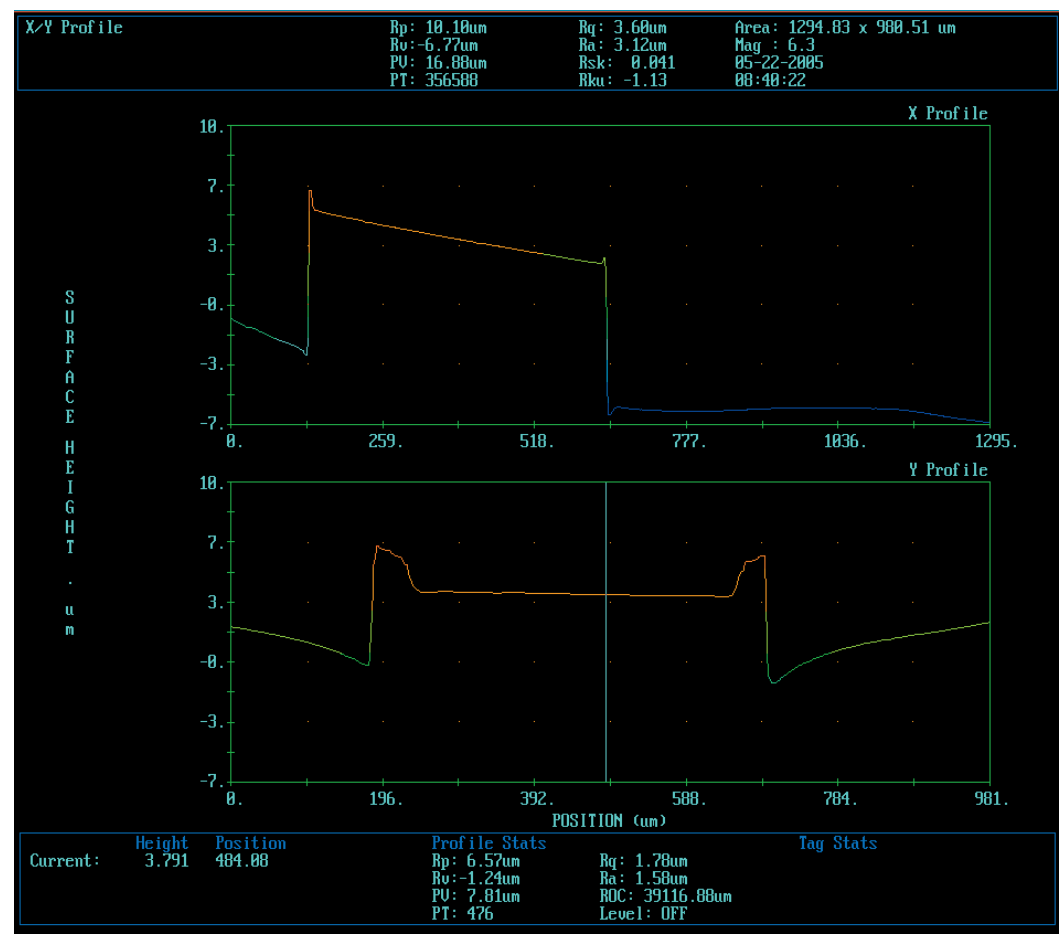

Plot of height through chamber adjacent to port (Point B on port contour plot):




Measurements from the chamber tip area:

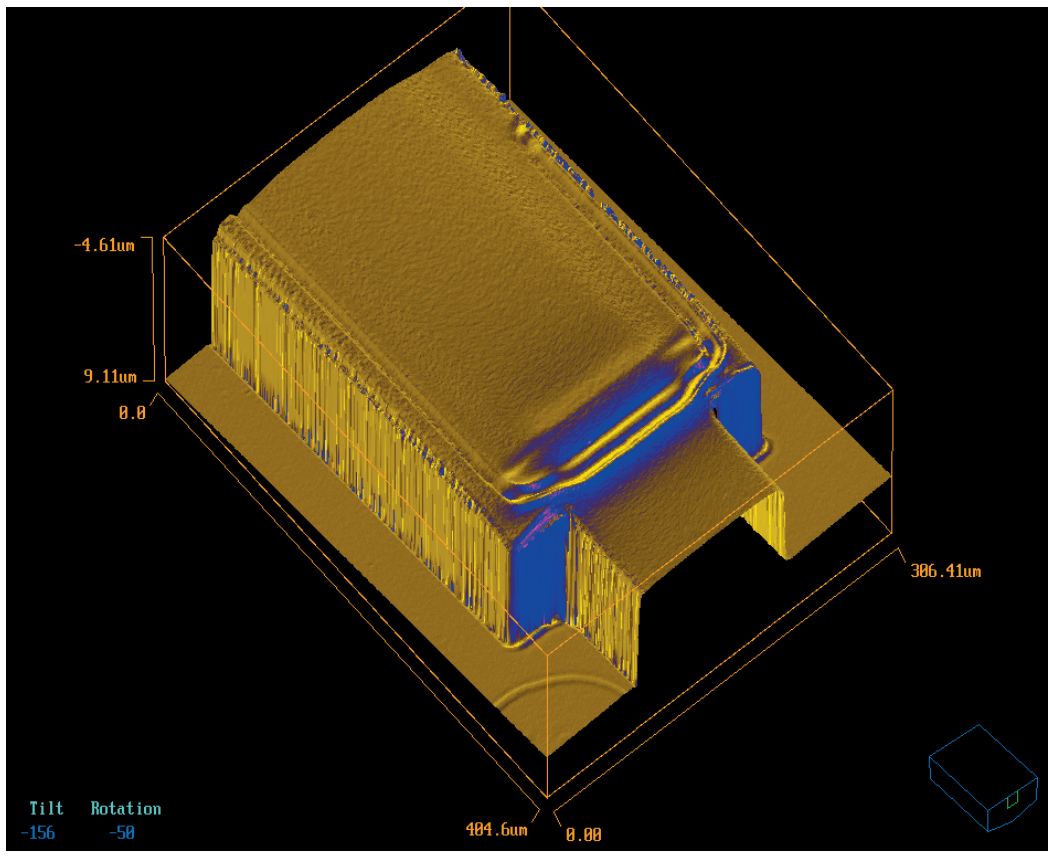

Contour plot of the tip area:




Plot of height through tip center (Point A on tip contour plot):

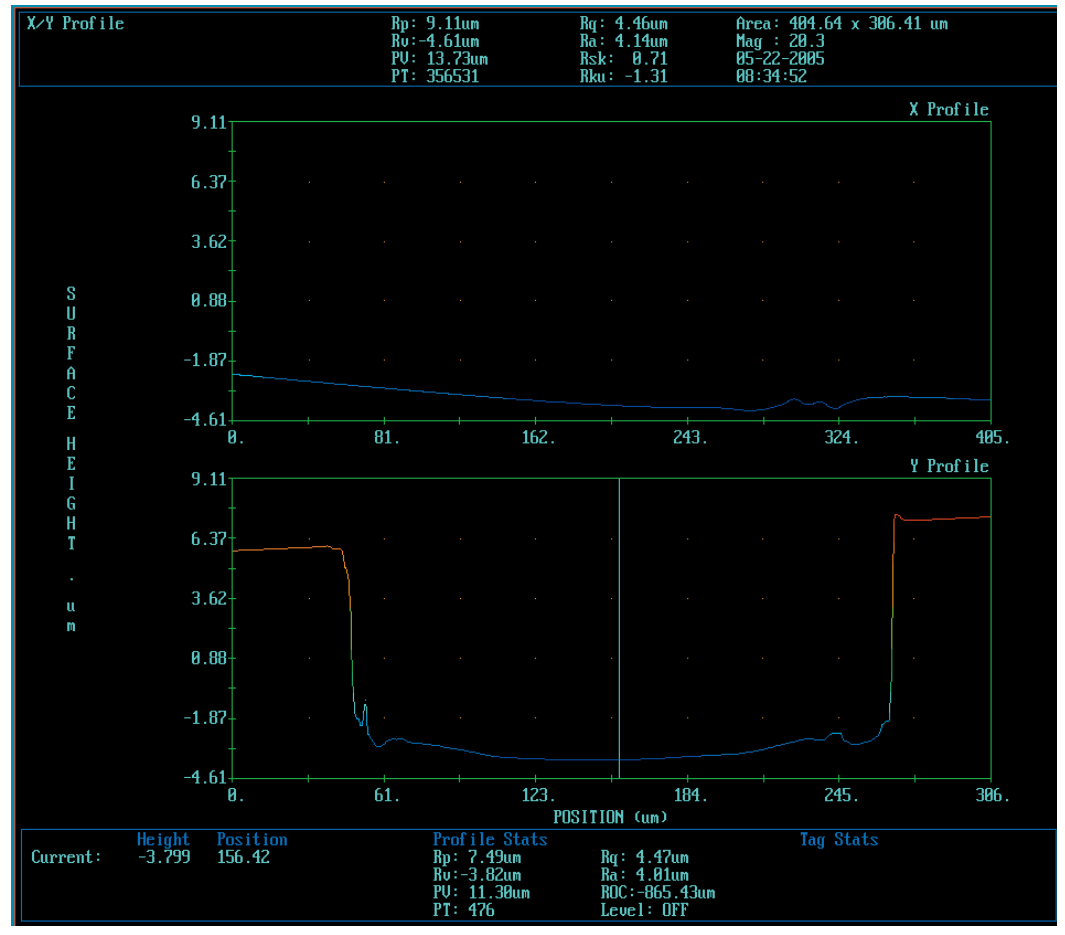

Plot of height through leash (Point B on tip contour plot):




Measurements from the handle area. 3D plot:



Contour plot of handle area:

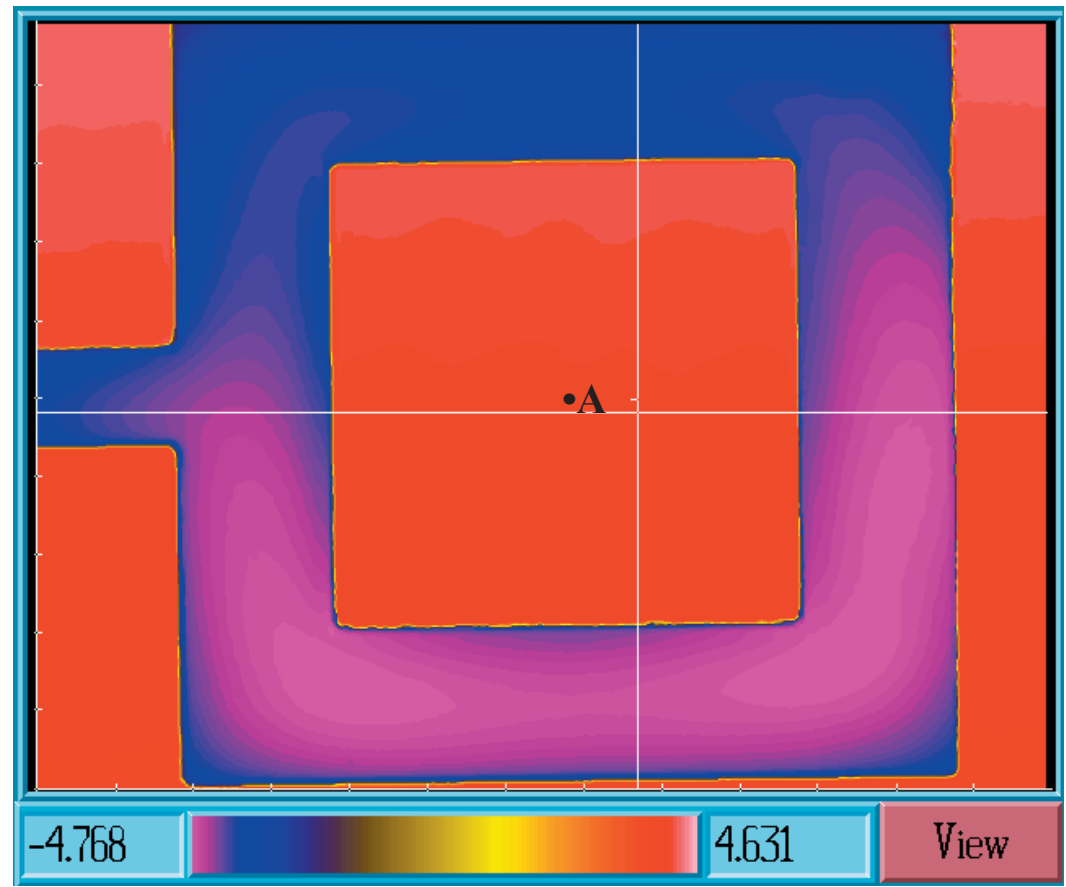


Plot of height through handle center (Point A on handle contour plot):



